Self-Assembled Epitaxial Multiferroic Oxide Nanostructures Grown by Pulsed Electron Deposition

A Dissertation
Presented to
the faculty of the School of Engineering and Applied Science
University of Virginia
in partial fulfillment
of the requirements for the degree
Doctor of Philosophy
by

Ryan Comes

May

2013 
APPROVAL SHEET

The dissertation

is submitted in partial fulfiliment of the requirements

for the degree of

Doctor of Philosophy

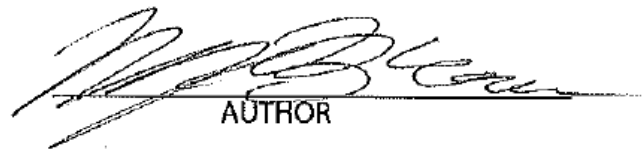

The dissertation has been read and approved by the examining committee:

\begin{tabular}{c} 
Stuart Wolf \\
\hline $\begin{array}{c}\text { Advisor } \\
\text { Jerrold Floro }\end{array}$ \\
\hline Jiwei Lu \\
\hline Mircea Stan \\
\hline Joseph Poon
\end{tabular}

Accepted for the School of Engineering and Applied Science:

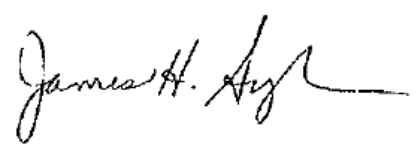

Dean, School of Engineering and Applied Science

May

2013 


\section{Self-Assmebled Epitaxial Multiferroic Oxide Nanostructures Grown by Pulsed Electron Deposition}

\section{Table of Contents}

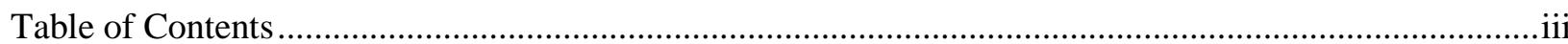

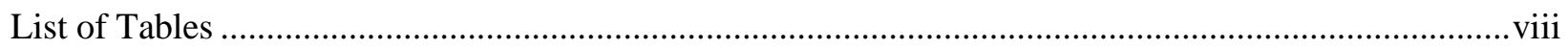

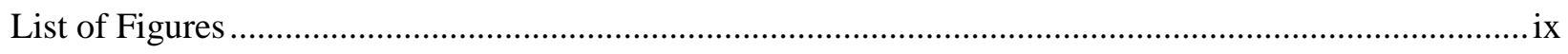

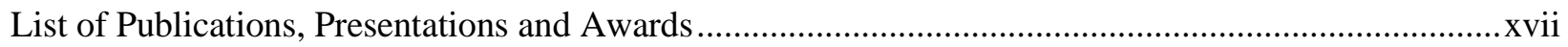

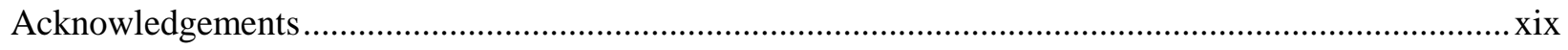

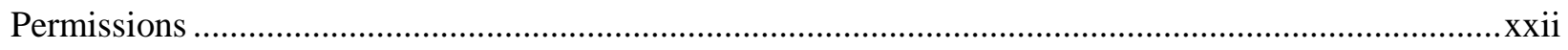

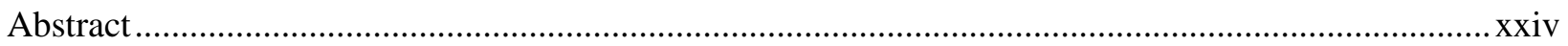

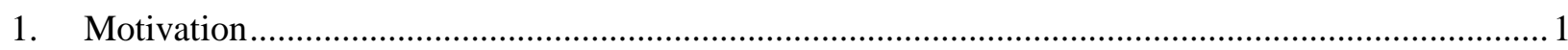

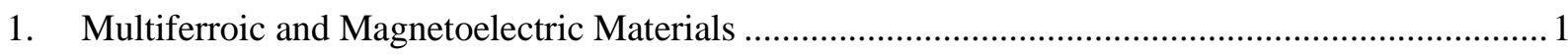

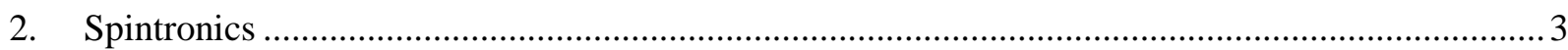

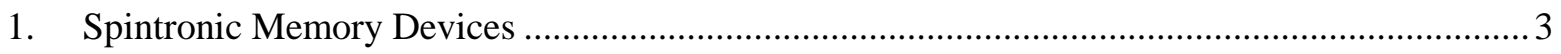

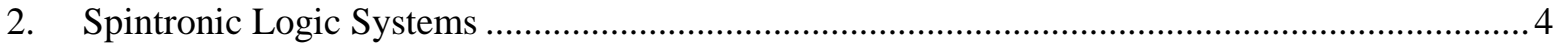

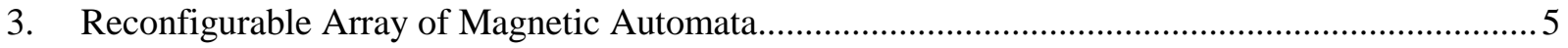

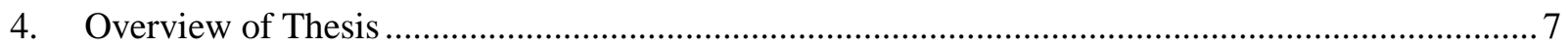

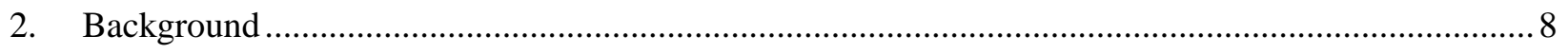




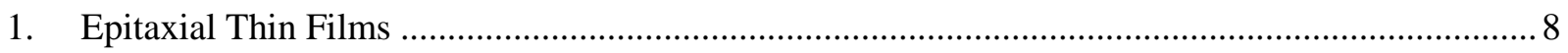

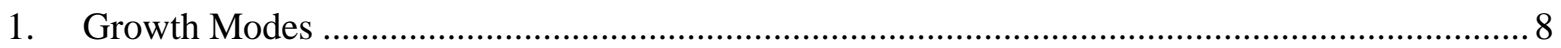

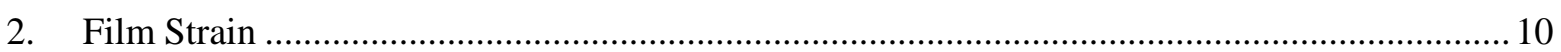

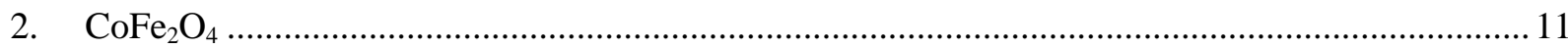

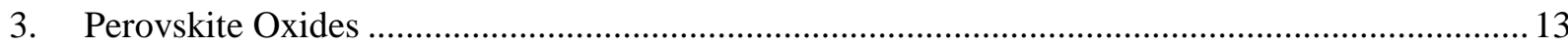

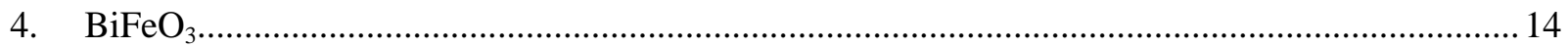

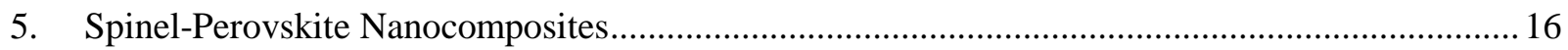

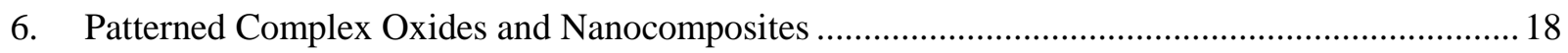

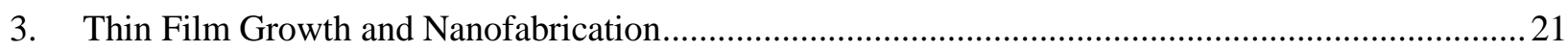

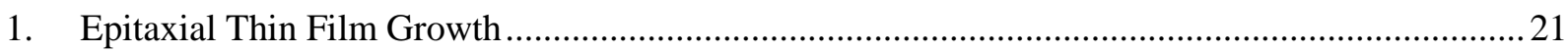

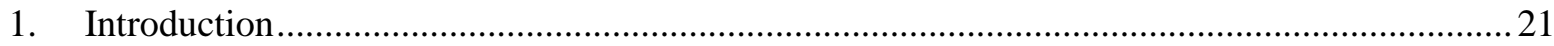

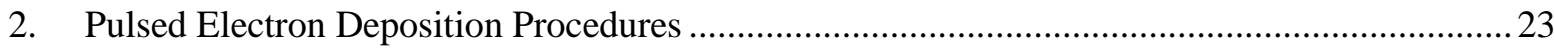

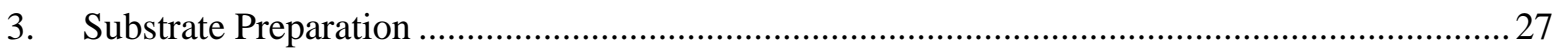

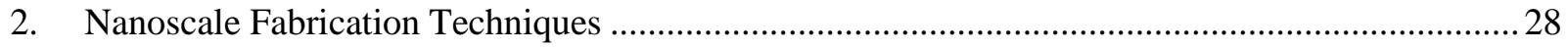

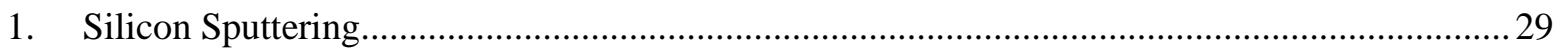

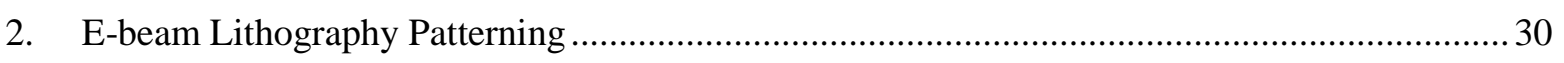

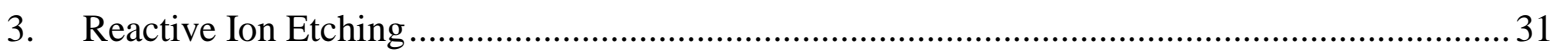

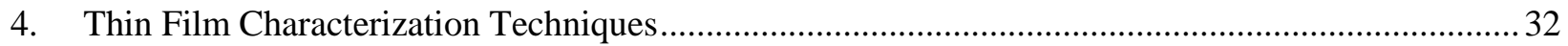

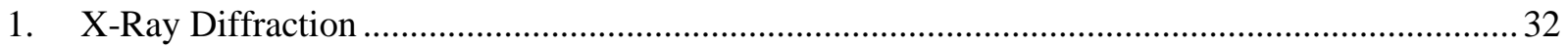

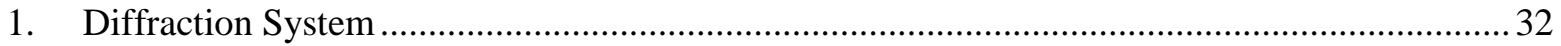

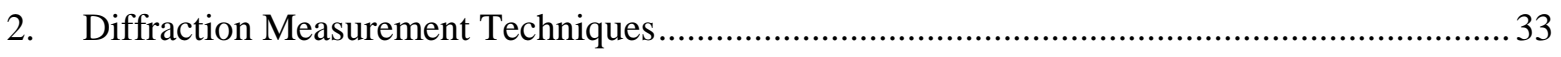




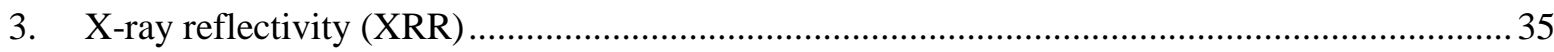

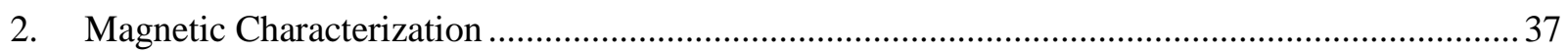

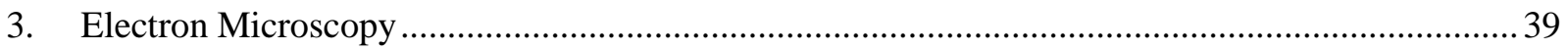

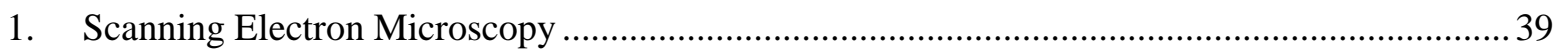

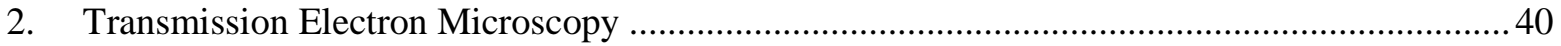

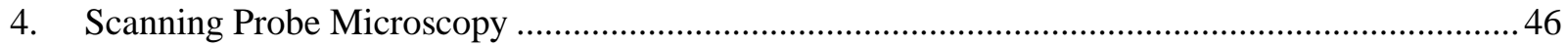

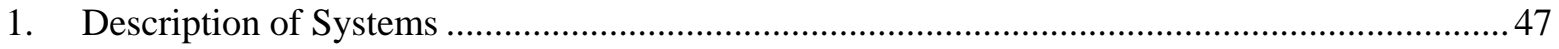

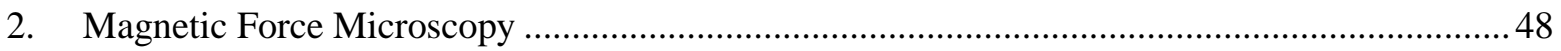

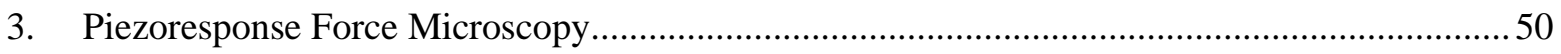

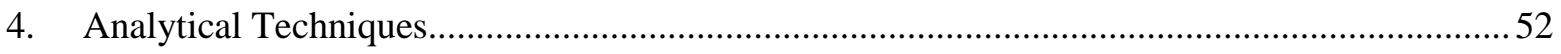

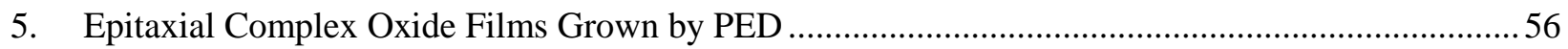

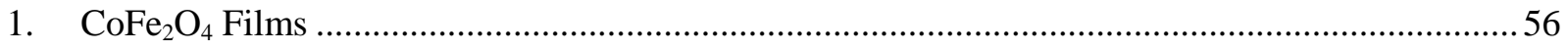

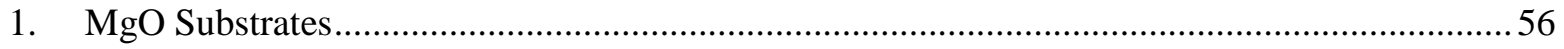

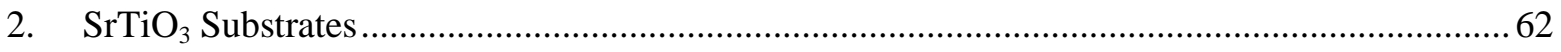

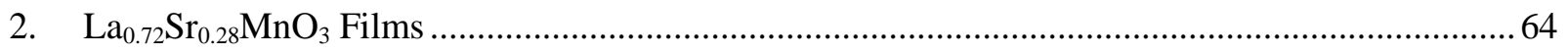

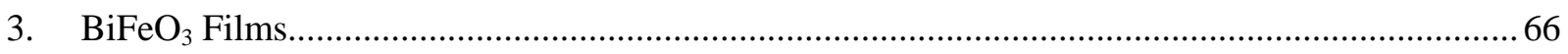

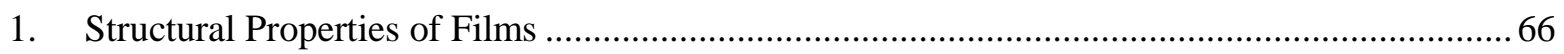

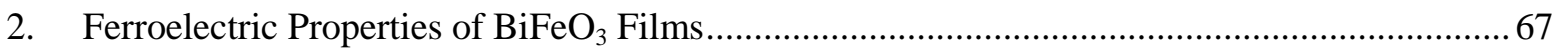

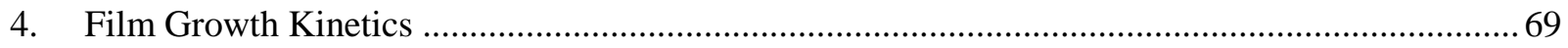

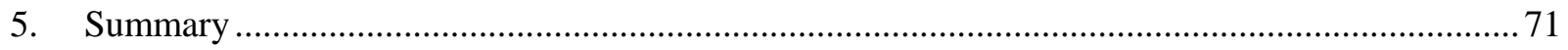

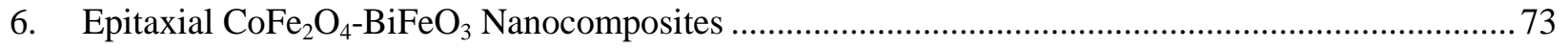




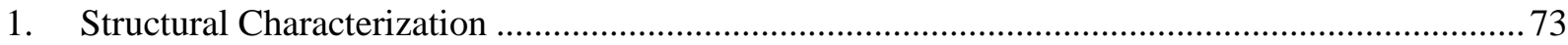

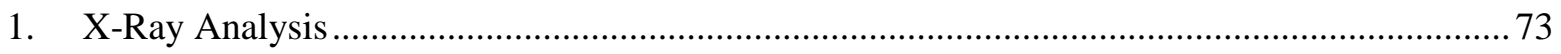

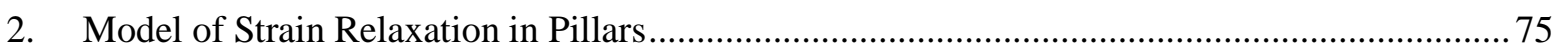

2. Transmission Electron Microscopy Analysis of Nanocomposite ................................................ 77

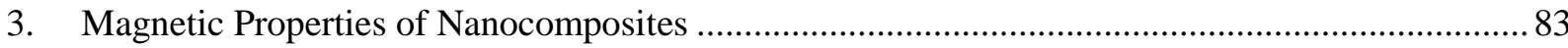

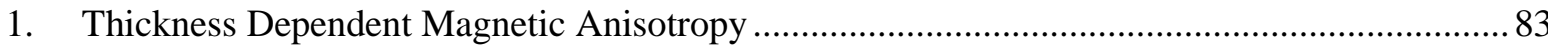

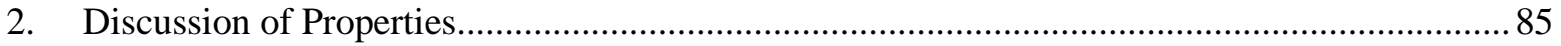

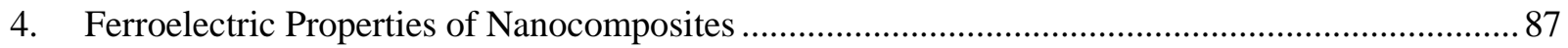

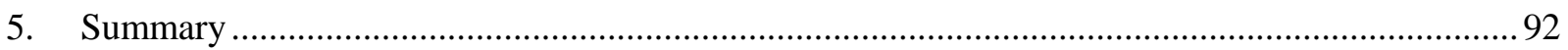

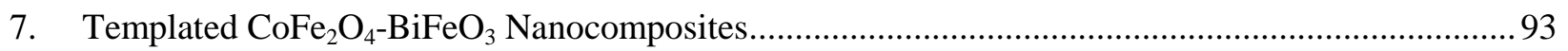

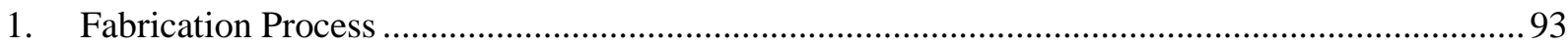

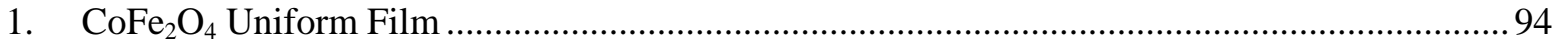

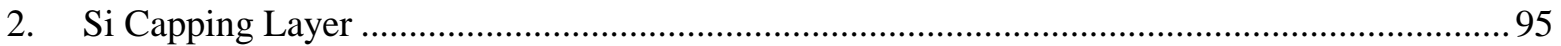

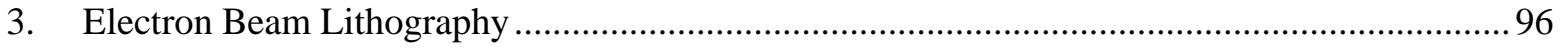

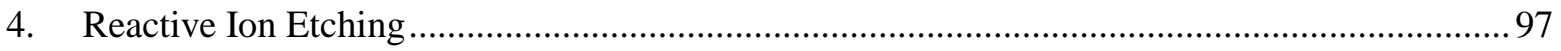

2. Structural Characterization of Templated Composites ....................................................... 100

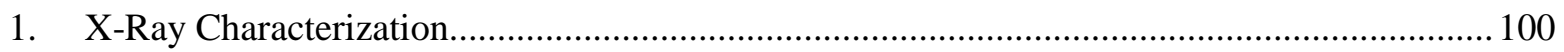

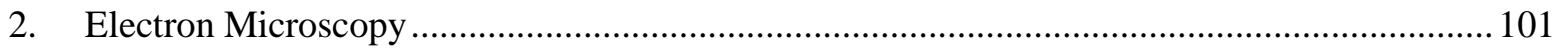

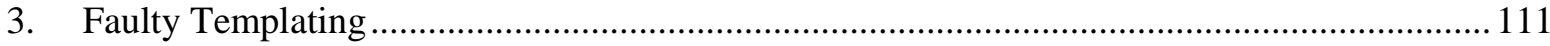

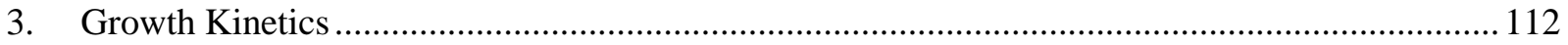

4. Magnetic Properties of Templated Nanocomposites .............................................................. 118 
5. Ferroelectric Properties of Templated Nanocomposites

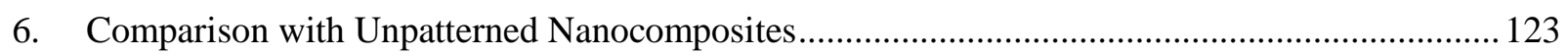

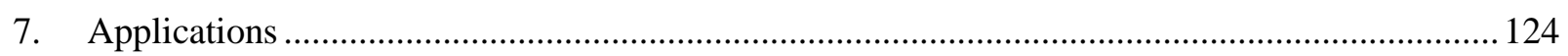

1. Reconfigurable Array of Magnetic Automata................................................................... 124

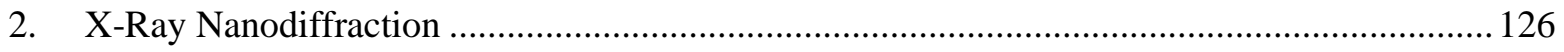

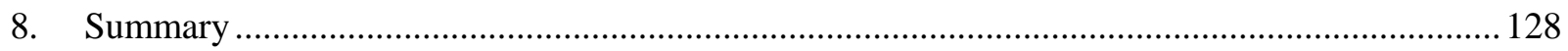

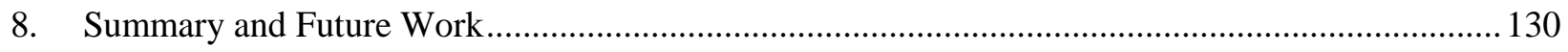

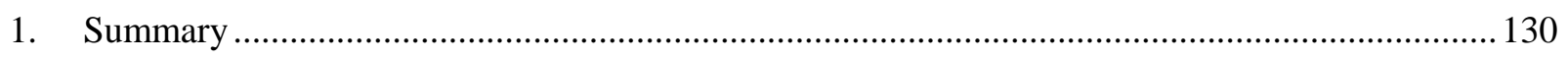

1. Growth of Complex Oxide Films via Pulsed Electron Deposition ........................................ 130

2. Spontaneously-formed $\mathrm{CoFe}_{2} \mathrm{O}_{4}-\mathrm{BiFeO}_{3}$ Epitaxial Nanocomposites ................................... 131

3. Directed Self-Assembly of $\mathrm{CoFe}_{2} \mathrm{O}_{4}-\mathrm{BiFeO}_{3}$ Nanocomposites ............................................ 131

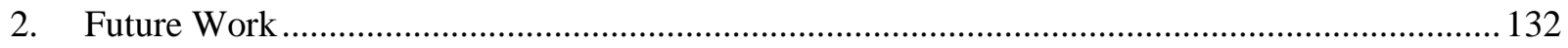

1. Growth of Complex Oxide Films via Pulsed Electron Deposition ........................................ 132

2. Ferroelectric Domain Wall Pinning in $\mathrm{CoFe}_{2} \mathrm{O}_{4}-\mathrm{BiFeO}_{3}$ Nanocomposites ............................ 133

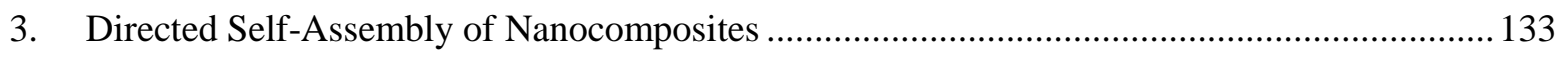

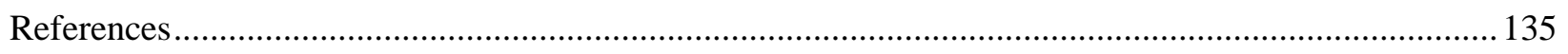

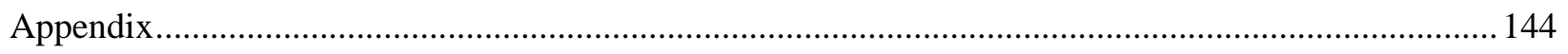




\section{List of Tables}

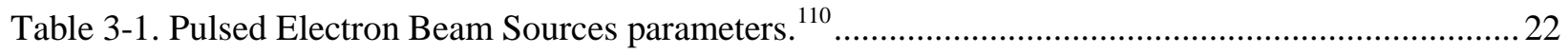

Table 5-1. Crystallographic and magnetic data for films in the study.................................................57

Table 6-1. Lattice parameters, strain conditions and anisotropy values for composite films in the study. 74

Table 7-1. Pillar Dimensions Measured via AFM in Figure 7-20...................................................... 115

Table 7-2. Published data for CFO pillar diameter and film growth rate taken from Zheng, et al. ${ }^{71}$

Calculated data for capture radius and monolayer formation time. 


\section{List of Figures}

Figure 1-1. A Venn diagram showing the relationship of multiferroic materials to the class of materials systems that exhibit magnetoelectric properties. Adopted from Eerenstein, et al. ${ }^{1}$. 2

Figure 1-2. Types of multiferroic films: (left) A single phase film such as $\mathrm{EuTiO}_{3}$ or the hexagonal manganites; (center) A bilayer film where interfacial coupling through effects such as exchange bias produces multiferroic behavior; (right) A vertical composite such as the spinel-perovskite nanocomposites that produce multiferroic coupling through strain. Adopted from Ramesh and Spaldin. ${ }^{2}$ 3

Figure 1-3. Schematic of Reprogrammable Array of Magnetic Automata (RAMA) logic architecture. A square array of CFO pillars with perpendicular magnetic anisotropy in a BFO matrix is produced. Individual pillars are addressed using wires above and below the array to write a ferroelectric domain in

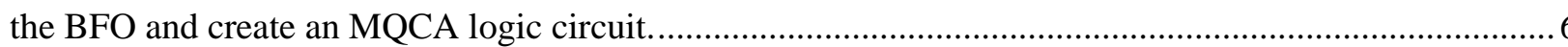

Figure 2-1. A schematic of the various growth modes possible during thin film growth.........................

Figure 2-2. Schematic showing the growth of a heteroepitaxial film with strain due to lattice mismatch. (left) For thicknesses less than $d_{c}$ the film is coherently strained to the substrate in plane lattice. (right) For thicknesses greater than $d_{c}$ misfit dislocations form with a dislocation density that is thickness dependent.

Figure 2-3. Rendering of a single unit cell of $\mathrm{CoFe}_{2} \mathrm{O}_{4}$ using VESTA. ${ }^{40} \mathrm{Fe}^{3+}$ ions occupy all tetrahedrally coordinated sites (brown) and half of the octahedrally coordinated sites (blue), while $\mathrm{Co}^{2+}$ ions occupy the remaining octahedral sites. 12

Figure 2-4. Rendering of perovskite unit cell using VESTA. ${ }^{40}$ Yellow ions represent A site ions, blue ions represent $\mathrm{B}$ site ions, and red ions are $\mathrm{O}^{2-}$ ions. The $\mathrm{O}^{2-}$ ions form an octahedron surrounding the $\mathrm{B}$ site ion.

Figure 2-5. (left) The unit cell of unstrained $\mathrm{BiFeO}_{3}$ in the rhombohedral space group. (center) The pseudocubic unit cell of rhombohedral $\mathrm{BiFeO}_{3}$ showing the octahedral tilt pattern. (right) The tetragonal unit cell of $\mathrm{BiFeO}_{3}$ showing the ionic displacements in the material. All images rendered in VESTA. ${ }^{40} . .15$ 
Figure 2-6. (a) Cross-sectional view of CFO pillar; (b) Top-down view of pillar.

Figure 3-1. Photographs of the PED system. (A) A view inside the chamber showing the left electron gun with the new metallic sleeve and the right electron gun with the older cooling coil holder, the substrate holder and heater, and the target stage. (B) An image of the plume produced during ablation of the $\mathrm{CoFe}_{2} \mathrm{O}_{4}$ target.

Figure 3-2. (A) Disassembled sleeve (top), tube (bottom) and alumina cap (left). (B) Assembled system.

Figure 3-3. Alumina holder (left) and ablated Bi1.2FeO3 target (right). Target is 1 inch in diameter.......25

Figure 3-4. Sample plate with substrates mounted using fast drying silver paint.. 26

Figure 3-5. Temperature calibration data showing substrate temperature as a function of setpoint temperature. ${ }^{123}$

Figure 3-6. As received SrTiO3 (A) and LaAlO3 (B) substrates showing multiple terminations and minimal surface roughness with visible step edges. Note the presence of twinning in the LaAlO3 substrate due to the rhombohedral room temperature crystal structure.

Figure 3-7. A) AFM topography scan for representative $\mathrm{SrTiO}_{3}$ substrate; B) Line profile along gray line in (A); C) AFM topography scan for representative $\mathrm{Nb}$-doped $\mathrm{SrTiO}_{3}$ substrate; D) Line profile along gray line in $(\mathrm{C})$

Figure 3-8. (a) Initial structure before patterning; (b) HSQ resist spun onto Si cap; (c) Resist is patterned to form dots using e-beam lithography; (d) Pattern is transferred through the Si cap using RIE; (e) Pattern is transferred through CFO film using Ar mill. The STO substrate is partially etched during this process.

Figure 4-1. Geometry of diffraction system for reciprocal space maps. Green angle marks indicate equivalent angles centered about the dashed line. .35

Figure 4-2. Screen capture of representative x-ray reflectivity fit for $\mathrm{BiFeO} 3$ film grown on $\mathrm{SrTiO}_{3}$, showing the critical angle and oscillation period. .37 
Figure 4-3. a) VSM coils used in the VersaLab and PPMS (left) $5 \mathrm{~mm}$ bore, (right) large bore for perpendicular measurements; (b) Example of sample mounted for in-plane VSM measurement; (c) Example of sample mounted for perpendicular VSM measurement.

Figure 4-4. Preparation process for TEM lamella. A) Deposition of Pt protective barrier along $\langle 110\rangle$ axis. B) Trench milling and Omniprobe attachment to lamella. C) Mounting of lamella on $\mathrm{Cu}$ grid. D) View of final thinned lamella. .42

Figure 4-5. Energy Dispersive X-Ray Spectroscopy (EDS) spectrum showing relevant X-ray peaks for $\mathrm{CoFe}_{2} \mathrm{O}_{4}-\mathrm{BiFeO}_{3}$ nanocomposite grown on $\mathrm{SrTiO}_{3}$ .46

Figure 4-6. Photograph of Asylum Cypher configuration for piezoresponse force microscopy (PFM) measurement. For non-PFM measurements, the bias lead was not used. .48

Figure 4-7. Representative applied bias signal for switching spectroscopy PFM measurements. .52 Figure 4-8. Screencapture from WSxM software showing the flattening process for an array of CoFe2O4 islands on the surface of SrTiO3. Note the bad scan line approximately $20 \%$ from the top in the filtered image marked with an arrow.

Figure 4-9. Screencapture from WSxM software showing the flooding process for an patterned nanocomposite with $\mathrm{CoFe}_{2} \mathrm{O}_{4}$ pillars in $\mathrm{BiFeO}_{3}$ matrix. .55

Figure 5-1. X-ray diffraction patterns of $\mathrm{MgO}$ (002) (left peak) and $\mathrm{CoFe}_{2} \mathrm{O}_{4}$ (004) (right peak) for films with thicknesses of $20 \mathrm{~nm}, 40 \mathrm{~nm}$, and $82 \mathrm{~nm}$ showing thickness fringes in all three cases. (Inset) Wide range scan for thickest film showing that no secondary phases are present.

Figure 5-2. Reciprocal space maps about the MgO (224) peak (bottom peak) for CFO films (top peak) with thicknesses: (A) $20 \mathrm{~nm}$, (B) $40 \mathrm{~nm}$, and (C) $82 \mathrm{~nm}$. Units of both $\mathrm{Q}_{\mathrm{x}}$ and $\mathrm{Q}_{\mathrm{z}}$ are $\AA^{-1}$, with $\mathrm{Q}_{\mathrm{x}}$ representing the [110] direction and $\mathrm{Q}_{\mathrm{z}}$ representing the [001] direction. .58 Figure 5-3. (a) AFM topography of $20 \mathrm{~nm}$ film; (b) MFM phase corresponding to (a); (c) AFM topography of $40 \mathrm{~nm}$ film; (d) MFM phase corresponding to (c); (e) AFM topography of $82 \mathrm{~nm}$ film; (f) MFM phase corresponding to (e). Note that the scale bars vary between images. Image scales were chosen to include a comparable number of domains in each image. .59 
Figure 5-4. Perpendicular (solid, blue) and in-plane (dotted, red) M vs. H hysteresis loops for all three films: (a) $20 \mathrm{~nm}$ film; (b) $40 \mathrm{~nm}$ film; (c) $82 \mathrm{~nm}$ film.

Figure 5-5. Atomic force microscopy topography scans of $\mathrm{CoFe}_{2} \mathrm{O}_{4}$ films grown at (a) $9 \mathrm{kV}$ and (b) 12 $\mathrm{kV}$, showing similar island growth modes but different particulate densities. Note the difference in scale between the images.

Figure 5-6. Atomic force microscopy topography scans of $\mathrm{La}_{0.72} \mathrm{Sr}_{0.28} \mathrm{MnO}_{3}$ films grown on (a) $\mathrm{SrTiO}_{3}$ and (b) $\mathrm{LaAlO}_{3}$ showing Frank-van der Merwe and Stranski-Krastanov growth modes. (c) A crosssectional line scan along the gray line shown in (b) showing the height of islands above the film surface.

Figure 5-7. Atomic force microscope topography images of (A) $2.1 \mathrm{~nm}$ thick LSMO film grown on STO substrate; (B) $4.4 \mathrm{~nm}$ thick LSMO film grown on STO substrate.

Figure 5-8. Atomic force microscopy topography scans of $\mathrm{BiFeO}_{3}$ films grown on (a) $\mathrm{SrTiO}_{3}$ and (b) $\mathrm{LaAlO}_{3}$. (c) X-ray diffraction measurement of the (001) and (002) substrate and film peaks for both films showing Kiessig fringes.

Figure 5-9. (a) X-ray diffraction measurement of $\mathrm{BiFeO}_{3}-\mathrm{La}_{0.72} \mathrm{Sr}_{0.28} \mathrm{MnO}_{3}$ bilayer grown on $\mathrm{SrTiO}_{3}$; (b) Piezoresponse force microscopy (PFM) topography scan of sample; (c) PFM out-of-plane phase scan of sample showing switched regions.

Figure 6-1. a) XRD measurement for Sample 2 (43 nm BFO thickness), with the CFO (008) peak inset and Gaussian curve fit to peak; b) AFM topography of Sample 2; c) Graph depicting in-plane (blue) and out-of-plane (red) strain conditions for samples examined in this section.

Figure 6-2. Models of strain relaxation (A) along the perpendicular axis and (B) along the in-plane <110> axes as the thickness of the nanocomposite increases. .77

Figure 6-3. (A) Out-of-plane x-ray diffraction measurement for $20 \mathrm{~nm}$ thick $\mathrm{BiFeO} 3-\mathrm{CoFe} 2 \mathrm{O} 4$ nanocomposite; (B) Reciprocal space map about SrTiO3 (103) peak; (C) In-plane and out-of-plane magnetic hysteresis loops of sample. .78

Figure 6-4. HAADF-STEM image of two nanopillars viewed along the <110> zone axis. .79 
Figure 6-5. EDS map of $\mathrm{CoFe}_{2} \mathrm{O}_{4}$ nanopillar embedded in $\mathrm{BiFeO}_{3}$ matrix. A) Overlay of all three elements mapped; B) Intensity of Co $K_{\alpha}$ peak; C) Intensity of Bi $L_{\alpha}$ peak; D) Intensity of Ti $K_{\alpha}$ peak. .... 81 Figure 6-6. A) HRTEM image of pillar measured via EDS in Figure 6-5. Arrows indicate examples of dark Moire fringes in pillar. B) Fast Fourier transform of entire field in A 82

Figure 6-7. HRTEM FFT analysis of substrate pillar and matrix of image in (A). Annotations in image show selected regions for FFT images in following images. B) Nb:STO substrate; C) BFO matrix; D) CFO pillar. Cubic or pseudocubic indices are noted in all three images with low index diffraction peaks. Green diamonds showing hexagonal pattern of peaks are shown as a guide to the eye in (C) and (D).... 83 Figure 6-8. a-c) Magnetic hysteresis loops for CFO-BFO composite film along in-plane [100] and out-ofplane [001] directions. a) Sample 1, with BFO thickness of $13 \mathrm{~nm}$; b) Sample 2, with BFO thickness of 43 $\mathrm{nm}$; c) Sample 3, with BFO thickness of $150 \mathrm{~nm} . \mathrm{d}$ ) Predicted magnetoelastic ( $\mathrm{K}_{\text {elastic }}$, square), shape ( $\mathrm{K}_{\text {shape }}$, triangle) and net $\left(\mathrm{K}_{\text {net }}\right.$, diamond) uniaxial anisotropy constants (left axis) and fields (right axis) for each film based on strain and pillar aspect ratios. Measured anisotropy fields $\left(\mathrm{K}_{\text {actual }}\right.$, circle $)$ are also shown. Rectangles are drawn with the pillar aspect ratio of each sample.

Figure 6-9. Magnetic force microscopy image of nanocomposite. A) Topography; B) Phase overlaid as color on three-dimensional rendering of topography. Red and blue represent positive and negative out-ofplane fields (arbitrary sign), while green is neutral. The circle highlights a pillar with in-plane magnetization, while the box highlights a pillar with perpendicular moment. 85 Figure 6-10. Piezoresponse force microscopy (PFM) measurements of $20 \mathrm{~nm}$ thick BiFeO3-CoFe2O3 nanocomposite made using Dual AC Resonance Tracking (A) Surface topography; (B) PFM amplitude; (C) PFM out-of-plane phase; (D) 3D rendering of the surface topography with the out-of-plane phase overlaid. Switching-spectroscopy PFM (E) amplitude and (F) phase measurements made at the X in (A).

Figure 6-11. Vector piezoresponse force microscopy (PFM) measurements made on the same sample as in YY. (A) Sample topography; Out-of-plane (B) amplitude and (C) phase; (D) In-plane phase overlaid on 
3D rendering of surface topography; In-plane (E) amplitude and (F) phase measurements with the same color maps as in the out-of-plane direction (arb. units for amplitude).

Figure 7-1. Flow chart for the fabrication process. (a) Deposition of $\mathrm{CoFe}_{2} \mathrm{O}_{4}$ (CFO) film using pulsed electron deposition (PED); (b) Deposition of amorphous Si capping layer using RF sputtering; (c) Spin coating of sample with HSQ negative-tone e-beam resist; (d) Patterning of pillars using e-beam lithography; (e) Reactive ion etching of Si cap; (f) Ar ion etching of CFO film; (g) Deposition of $1 \mathrm{~nm}$ thick $\mathrm{BiFeO}_{3}$ (BFO) film using PED; (h) Co-deposition of CFO and BFO using PED to form an epitaxial nanocomposite. .94

Figure 7-2. Atomic force microscope (AFM) image of uniform CFO film used for template pattern showing regular epitaxial islands on the surface. .95

Figure 7-3. (A) Out-of-plane x-ray diffraction data for $\mathrm{Si} / \mathrm{CoFe}_{2} \mathrm{O}_{4} / \mathrm{Nb}: \mathrm{SrTiO}_{3}$ heterostructure. The peak at $\sim 43^{\circ}$ is the $\mathrm{CFO}(004)$ peak, while the other two peaks are substrate peaks. (B) X-ray reflectivity data for same structure.

Figure 7-4. Scanning electron microscope image of EBL-patterned HSQ pillars on the surface of the Si capping layer. Scale bar represents $200 \mathrm{~nm}$.

Figure 7-5. Atomic force microscope topography image of island array with $200 \mathrm{~nm}$ pitch after RIE showing atomic terraces on substrate surface.

Figure 7-6. Array of template islands patterned for sample characterized via electron microscopy. A) Atomic force microscopy topography image of array; B) Height profile along gray line in (A). Figure 7-7. Reciprocal space map of sample showing the (103) diffraction peak of the $\mathrm{Nb}$-doped $\mathrm{SrTiO}_{3}$ substrate (top) and pseudocubic (103) peak of the $\mathrm{BiFeO}_{3}$ matrix (bottom). 101

Figure 7-8. Scanning electron microscope image of templated array of $\mathrm{CoFe} 2 \mathrm{O} 4$ nanopillars in $\mathrm{BiFeO} 3$ matrix with $100 \mathrm{~nm}$ pitch. Horizontal axis is parallel to [100] direction. 102 Figure 7-9. HAADF-STEM image of patterned nanocomposite. A) Wide image showing uniform spacing of pillars; B) Image of left pillar in (A); C) Image of right pillar in (A). 103 
Figure 7-10. Energy dispersive x-ray spectroscopy map of pillar from Figure 7-9(C). A) HAADF signal at each pixel measured; B) Bi $L_{\alpha}$ edge map; C) Co $K_{\alpha}$ edge map; D) Ti $K_{\alpha}$ edge map....... 105 Figure 7-11. A) Overlay of EDS map images from Figure 7-10. b) Overlaid images from high resolution EDS map of pillar-substrate interface. 105

Figure 7-12. TEM images of pilar from Figure 7-9(C). (A) Wide view (scale bar represents $10 \mathrm{~nm}$ ); (B) Tight view showing Moire fringing due to incoherent pillar-matrix along zone axis (scale bar represents 2 $\mathrm{nm})$; (C) View of pillar facet above surface of matrix (scale bar represents $2 \mathrm{~nm}$ ). 107 Figure 7-13. HRTEM images of matrix-pillar (A) and pillar-substrate (B) interfaces. C) Fourier-filtered image of (A) used to examine coherency of matrix-pillar interface. D) Fourier-filtered image of (B) used to examine pillar-substrate interface. Scale bars in (A) and (B) represent $2 \mathrm{~nm}$. 108

Figure 7-14. FFT analysis of pillar-substrate interface, with regions of interest outlined in the image to the left. A) FFT image of pillar; B) FFT image of substrate. 109

Figure 7-15. FFT analysis of pillar-matrix interface, with regions of interest outlined in the image to the left. A) FFT analysis of matrix; B) FFT analysis of pillar. 109

Figure 7-16. High-resolution TEM images at two diffrent magnifications showing a pillar formed on top of an island comprised of some of the $\mathrm{Nb}$ :STO substrate. A) 115,000x magnfication; B) 245,000x magnfication with annotations showing the substrate surface (green) and $3 \mathrm{~nm}$ tall $\mathrm{Nb}$ :STO mound (red) that is formed beneath the CFO island.

Figure 7-17. High-resolution TEM image at 380,000x magnification of the same pillar as BLAH (A), with false color overlay (B) showing the estimated interface boundaries between $\mathrm{Nb}: \mathrm{SrTiO}_{3}$ (blue), $\mathrm{BiFeO}_{3}$ (green) and $\mathrm{CoFe}_{2} \mathrm{O}_{4}$ (red). 111

Figure 7-18. Scanning electron microscope image of array exhibiting faulty templating. Note that properly templated pillars form at the corner of the array due to variation in the electron beam lithography process near the edges of the array. 112 Figure 7-19. Scanning electron microscope image of arrays with three different pitch sizes patterned on the same substrate. 113 
Figure 7-20. (a) Pillar topography with $100 \mathrm{~nm}$ center-to-center distance (pitch); (b) Pillar topography with $150 \mathrm{~nm}$ pitch; (c) Pillar topography with $200 \mathrm{~nm}$ pitch, with defect CFO pillar highlighted; (d-f) Cross section data for: (d) $100 \mathrm{~nm}$, (e) $150 \mathrm{~nm}$, and (f) $200 \mathrm{~nm}$ AFM images along the black lines shown in $(\mathrm{a}-\mathrm{c})$.

Figure 7-21. Magnetic force microscopy analysis of $150 \mathrm{~nm}$ pitch array. a) Schematic of possible pillar magnetization contrasts; b) Topography image; c) MFM phase image; d) 3-dimensional representation of pillar topography with phase overlaid as color. Arrows in (d) represenent the observed magnetization directions for selected pillars.

Figure 7-22. (a) Contact mode topography image taken during piezoresponse force microscopy (PFM) measurement (' $\mathrm{X}$ ' indicates the site of switching spectroscopy-PFM (SS-PFM) measurement in Fig. 6); (b) Ferroelectric amplitude image corresponding to (a) (dark indicates low PFM response); (c) Ferroelectric phase image corresponding to (a) (white and orange correspond to oppositely oriented domains along the out-of-plane direction).

Figure 7-23. (a) Out-of-plane ferroelectric phase loop in $\mathrm{BiFeO} 3$ (BFO) matrix measured using piezoresponse force microscopy (PFM); (b) Measured tip displacement showing the expected butterfly loop behavior.

Figure 7-24. Vector piezoresponse force microscopy measurement of templated sample. A) Topography; B) Vertical amplitude response; C) Vertical phase response; D) Lateral amplitude response; E) Lateral phase response.

Figure 7-25. Lithographic patterning of BFO domain structure via piezoresponse force microscopy. A) Topography of nanopillar array; B) Applied bias map; C) PFM amplitude image following poling; D) PFM phase image after poling. . 126

Figure 7-26. A) Nanoscale map of the centroid of the BFO diffraction peak, showing that the lattice parameter of the BFO film is different in the area of the patterned array than in the unpatterned regions; B) CCD image of diffracted peak at the BFO $(002)_{\mathrm{pc}}$ Bragg condition, showing coherent scattering that may be due to the presence of a pillar. 128 


\section{List of Publications, Presentations and Awards}

During the course of my research at the University of Virginia, I have been able to publish several articles as the first author based on the work that went into this thesis. A list of these publications, along with two in preparation manuscripts, is provided below.

1. Ryan Comes, Hongxue Liu, Kerry Siebein, Jiwei Lu and Stuart A. Wolf. "In-plane Domain Wall Pinning in $\mathrm{CoFe}_{2} \mathrm{O}_{4}-\mathrm{BiFeO}_{3}$ Epitaxial Nanocomposites." In preparation.

2. Ryan Comes, Kerry Siebein, Hongxue Liu, Jiwei Lu and Stuart A. Wolf. "Chemical Templating of Self-Assembled Epitaxial Spinel-Perovskite Nanocomposites.” In preparation.

3. Ryan Comes, Man Gu, Mikhail Khokhlov, Hongxue Liu, Jiwei Lu and Stuart A. Wolf. "Electron Molecular Beam Epitaxy: Layer-by-layer Growth of Complex Oxides via Pulsed Electron-beam Deposition," Journal of Applied Physics, 113 (2), 023303, Jan 2013.

4. Ryan Comes, Hongxue Liu, Mikhail Khokhlov, Richard Kasica, Jiwei Lu and Stuart A. Wolf. "Directed Self-Assembly of Epitaxial $\mathrm{CoFe}_{2} \mathrm{O}_{4}-\mathrm{BiFeO}_{3}$ Nanocomposites," Nano Letters, 12 (5), 2367-2373, May 2012.

5. Ryan Comes, Mikhail Khokhlov, Hongxue Liu, Jiwei Lu and Stuart Wolf. "Magnetic anisotropy in composite $\mathrm{CoFe}_{2} \mathrm{O}_{4}-\mathrm{BiFeO}_{3}$ ultrathin films grown by pulsed-electron deposition," Journal of Applied Physics,111 (7), Apr 2012.

6. Ryan Comes, Man Gu, Mikhail Khokhlov, Jiwei Lu and Stuart A. Wolf. "Microstructural and domain effects in epitaxial $\mathrm{CoFe}_{2} \mathrm{O}_{4}$ films on $\mathrm{MgO}$ with perpendicular magnetic anisotropy," Journal of Magnetism and Magnetic Materials, 324 (4), 524-527, Feb 2012.

The research in this work has also been presented at a variety of national conferences. A list of these presentations in which I was the lead author is provided below.

1. Ryan Comes. "Directed Self-Assembly of Multiferroic Oxide Nanocomposites for Use in Magnetic Logic Architecture.” Materials Research Society Spring Meeting 2013, April 4, 2013.

2. Ryan Comes, Mikhail Khokhlov, Hongxue Liu, Jiwei Lu, and Stuart Wolf. "Self-Assembled Multiferroic Nanocomposites for Use in Magnetic Logic Architecture." American Physical Society March Meeting 2013, March 19, 2013.

3. Ryan Comes, Mikhail Khokhlov, Hongxue Liu, Jiwei Lu, and Stuart Wolf. "Magnetic and ferroelectric properties of patterned multiferroic $\mathrm{CoFe}_{2} \mathrm{O}_{4}-\mathrm{BiFeO}_{3}$ nanocomposites." American Physical Society March Meeting 2012, Feb 28, 2012

4. Ryan Comes, Hongxue Liu, Mikhail Khokhlov, Jiwei Lu, and Stuart A. Wolf. "E-beam Lithography Directed Self-assembly of $\mathrm{CoFe}_{2} \mathrm{O}_{4}-\mathrm{BiFeO}_{3}$ Nanocomposites." Materials Research Society Fall Meeting 2011, Nov 29, 2011. 
5. Ryan Comes, Mikhail Khokhlov, Hongxue Liu, Jiwei Lu, and Stuart A. Wolf. "Magnetic anisotropy in composite $\mathrm{CoFe}_{2} \mathrm{O}_{4}-\mathrm{BiFeO}_{3}$ ultrathin films grown by pulsed-electron deposition." 56th Annual Conference on Magnetism and Magnetic Materials 2011, Nov 3, 2011.

6. Ryan Comes, Man Gu, Mikhail Khokhlov, Jiwei Lu, and Stuart A. Wolf. " $\mathrm{CoFe}_{2} \mathrm{O}_{4}$ Nanostructures for Spintronic Logic Applications.” American Physical Society March Meeting 2011, March 22, 2011.

7. Ryan Comes, Richard Kasica, Gerard Henein, Jiwei Lu, and Stuart Wolf. "E-beam lithography patterning of Co/Pd multilayer pillars below $50 \mathrm{~nm}$ in diameter." American Physical Society March Meeting 2010, Mar 15, 2010.

I have been honored to receive several awards, fellowships and grants for my research and other activities while at the University of Virginia. A list of these awards is included below.

1. Materials Research Society, Graduate Student Silver Award, April 2013.

2. University of Virginia School of Engineering and Applied Science Graduate Teaching Fellow. August 2012.

3. University of Virginia Presidential Research Poster Competition, Finalist, May 2012.

4. University of Virginia Department of Materials Science and Engineering, Doris KuhlmnanWilsdorf Outstanding Graduate Student Award, May 2012

5. University of Virginia Engineering Research Symposium, First Place, April 2012.

6. Beamtime Award: Ryan Comes, Stuart Wolf, Jiwei Lu and Hongxue Liu. "X-ray microdiffraction measurement of ferroelectric-domain-induced strain in multiferroic nanocomposites." Argonne National Laboratory, Center for Nanoscale Materials, Nanoprobe Beamline. March 2012.

7. University of Virginia Engineering Research Symposium, Fifth Place, April 2011.

8. National Defense Science and Engineering Graduate Fellowship, August 2010-May 2013.

9. National Science Foundation, Graduate Research Fellowship Program, Honorable Mention, April 2009 


\section{Acknowledgements}

The work that has gone into producing this dissertation at the University of Virginia has been a long and at times challenging process, requiring support and assistance from family, friends, mentors and collaborators. To each of these people I owe a debt of gratitude for their contributions. I have thoroughly enjoyed my time at Virginia, even as I was struggling through the challenging times, and it is because of these people that things have worked out so well in the end.

I would first like to thank my advisors, Prof. Stuart Wolf and Prof. Jiwei Lu, for their guidance throughout my time at Virginia. They have been extremely supportive and encouraging, each providing valuable feedback on my work and guidance in tackling problems in the lab. Their guidance and recommendations during my recent job search have made the process far less difficult. I have learned a lot from their leadership of the group and hope that it will make me a better leader down the road. Thanks to both of you for all that you have done!

To all the members of the Wolf/Lu research group past and present, I would like to thank you for your collaboration, assistance, and friendship. You have all been very generous, teaching me how to use equipment, discussing science questions, and helping me with experiments all these years. To Kevin, Wei, David, Melissa, Wenjing, Wenbin, Yonghang, Mandy, Yishen, Lin, Tom, Colin, Yuhan, Hongxue and Nam: Thanks! I would also like to specifically thank Mikhail Khokhlov for his friendship and assistance as an undergraduate student in our lab for the past three summers. It has been great to watch him learn and grow as a scientist. Thanks for all of your help and good luck down the road as you start your own journey to grad school.

The faculty members on my thesis committee have been incredibly helpful in guiding me as a scientist. Prof. Joe Poon gave insightful comments during the proposal process that improved my work leading up to the completion of the thesis. Prof. Mircea Stan has been an ideal collaborator, keeping our group focused on the device applications for this research and offering kind advice on my career goals. I 
have worked very closely with my committee chairman, Prof. Jerry Floro, on both scientific and community outreach projects. His commitment to performing science well, while also helping students and bringing science to the masses through outreach events is rare and something I aspire to in the future. Thanks to all of you for your mentorship, guidance and critiques of my work!

Other members of the Materials Science and Engineering department have made the experience at Virginia a great one. I would like to thank Prof. Bill Johnson for serving as my teaching mentor during my teaching fellowship and offering so much guidance throughout the semester. The graduate teaching fellowship was one of the highlights of my time here and I could not have asked for a better mentor. Bill's considerate work with the Graduate Student Board also went a long ways to improving the group and the atmosphere in the department. I would also like to thank Vickie Thomas, Jeannie Reese, Susan Bagby, Sherri Sullivan, Beth Beal, Tonya Reynolds, Kim Fitzhugh-Higgins and Eric Newsome for their help with all the administrative hurdles that grad students must deal with. You have all been very kind and helpful!

Numerous outside collaborators have helped me with experiments and analysis that became a part of this thesis. At the National Institute of Standards and Technology (NIST) Center for Nanoscale Science and Technology (CNST), I owe thanks to scientific staff and administrative staff for their generous support of this work. Vince Luciani, Jeff Pasternak and Wade Hall have made my regular visits to the Nanofab as easy as possible and run a fantastic program. Dr. Gerard Henein trained and assisted me on the sputter deposition system in the cleanroom and was unfailingly kind and helpful at all of my visits to NIST. Dr. Kerry Siebein gave me invaluable assistance and collaboration in sample preparation and TEM analysis in the brief time that we worked together. Rich Kasica's assistance with the electron-beam lithography (EBL) systems in the Nanofab has been critical to the results that I have gotten. Without his assistance, many of the results that I have gotten would not have been possible. Rich has also been a good friend, chatting about all kinds of topics as we ran the EBL. To everyone at NIST, I would like to express my sincere appreciation for their assistance and support the past few years. 
Other collaborators have included Dr. Martin Holt and Dr. Stephan Hruszkewycz at Argonne National Lab. Martin and Stephan were both very friendly and helpful in designing and conducting the $\mathrm{x}$ ray nanodiffraction experiments that will be presented in this work. Thanks to both of them. I would also like to thank Prof. Caroline Ross and Nicolas Aimon of MIT for their helpful discussions and ongoing collaborations on the project.

I would be remiss if I didn't thank all of my friends at Virginia who have made grad school so fantastic. These folks have been classmates, collaborators, confidants and a great group of people to hang out with away from the office. Thanks to: Justin, Theron, Karen, Chris P., Chris N., Chris D., Kiril, Matt, Brandon, Sarah, Harmonie, Brad, Toni, and Jeremy. Good luck to all of you as you pursue (or are already at) the next stop in your career!

Last, but certainly not least, I would like to thank my family, along with my girlfriend Sara. All of you have been there to support me in so many ways throughout grad school. I love all of you and appreciate all of the support through the years. I would not be where I am today without the encouragement, guidance and emotional support of my family growing up and grad school would not have been the same without Sara at my side the past few years. Thanks! 


\section{Permissions}

Portions of this dissertation have been excerpted from previously published research articles written by Ryan Comes as the lead author. In each case, permission for the excerpting of the articles has been obtained from the publisher through the Copyright Clearance Center RightsLink system. Citations to the relevant works have been included in the body of the text. The following permissions were granted for this work, which has been excerpted and included in a variety of locations throughout the document:

1. Reprinted from:

Ryan Comes, Man Gu, Mikhail Khokhlov, Jiwei Lu and Stuart A. Wolf. "Microstructural and domain effects in epitaxial $\mathrm{CoFe}_{2} \mathrm{O}_{4}$ films on $\mathrm{MgO}$ with perpendicular magnetic anisotropy," Journal of Magnetism and Magnetic Materials, 324 (4), 524-527, Feb 2012, with permission from Elsevier.

License numbers: 3105920554489 for 2 excerpts of up to 1000 words and 3105920489998 for 3 figures.

2. Reprinted with permission from: Ryan Comes, Mikhail Khokhlov, Hongxue Liu, Jiwei Lu and Stuart A. Wolf. "Magnetic anisotropy in composite $\mathrm{CoFe}_{2} \mathrm{O}_{4}-\mathrm{BiFeO}_{3}$ ultrathin films grown by pulsed-electron deposition," Journal of Applied Physics, 111 (7), Apr 2012, American institute of Physics.

License number: 3105920245405 for an excerpt of greater than 800 words and associated figures

3. Reprinted (adapted) with permission from: 
Ryan Comes, Hongxue Liu, Mikhail Khokhlov, Richard Kasica, Jiwei Lu and Stuart A. Wolf.

"Directed Self-Assembly of Epitaxial $\mathrm{CoFe}_{2} \mathrm{O}_{4}-\mathrm{BiFeO}_{3}$ Nanocomposites," Nano Letters, 12 (5), 2367-2373, May 2012. Copyright 2012 American Chemical Society.

4. Reprinted with permission from:

Ryan Comes, Man Gu, Mikhail Khokhlov, Hongxue Liu, Jiwei Lu and Stuart A. Wolf. "Electron

Molecular Beam Epitaxy: Layer-by-layer Growth of Complex Oxides via Pulsed Electron-beam

Deposition," Journal of Applied Physics, 113 (2), 023303, Jan 2013, American Institute of

Physics.

License number: 3105920307421 for an excerpt of greater than 800 words and associated figures

Two figures for background information have been adapted from the published works of others.

Permission for use of these figures has been granted from their respective publishers.

1. Figure 1-1 in this work: Reprinted with permission from Macmillan Publishers Ltd: Eerenstein, W., Mathur, N. D. \& Scott, J. F. "Multiferroic and magnetoelectric materials." Nature 442, 759765 (2006).

License number: 3105920931434 . Figure 1 in the original article.

2. Figure 1-2 in this work: Reprinted with permission from Macmillan Publishers Ltd: Ramesh, R. \& Spaldin, N. A. "Multiferroics: progress and prospects in thin films." Nat Mater 6, 21-29 (2007).

License number: 3105921055767 . Figure 2 in the original article. 


\section{Abstract}

Multiferroic oxide nanocomposites thin films composed of a ferrimagnetic spinel oxide nanopillar and ferroelectric perovskite oxide matrix offer magnetoelectric coupling between the matrix and pillar, which can be used in spintronic devices for logic and memory applications. $\mathrm{CoFe}_{2} \mathrm{O}_{4}(\mathrm{CFO})$ offers unique properties as a magnetoelectric material due to its large magnetoelastic response under stain. Previous work has shown that when $\mathrm{CFO}$ is co-deposited with $\mathrm{BiFeO}_{3}(\mathrm{BFO})$, nanostructured phase segregation occurs, with CFO pillars forming in a BFO matrix. This work has also shown that electrical control of the magnetic anisotropy in the CFO pillar is possible via switching polarization of the ferroelectric BFO matrix. However potential spintronic device schemes require the ability to control the growth of these materials to pattern the locations where CFO pillars form, which has thus far been illusory.

This dissertation has focused on the templated growth of multiferroic complex oxide films and nanostructures via pulsed electron deposition (PED). The relatively new PED technique is similar to the more traditional pulsed laser deposition (PLD) approach, but with some key differences in growth kinetics. Uniform epitaxial CFO, BFO and $\mathrm{La}_{0.72} \mathrm{Sr}_{0.28} \mathrm{MnO}_{3}$ films have been grown on various substrates via the PED technique. All three epitaxial growth modes have been demonstrated in these materials, representing the first such result for complex oxide films grown via PED. Spontaneously formed epitaxial CFO-BFO nanocomposites have also been grown via PED and demonstrate unusual strain conditions in the CFO pillars that is not seen in composites grown via PLD. Analysis of the magnetic anisotropy of CFO-BFO nanocomposites using vibrating sample magnetometry showed that the magnetic properties what is expected given the strain demonstrated in the pillars. Finally, patterning of the growth of CFOBFO nanocomoposites has been demonstrated for the first time through the patterning of nanoscale CFO islands on the surface of the substrate to serve as a template for the composite. Electron and scanning probe microscopy analysis has demonstrated that these patterned composites exhibit similar crystal quality and multiferroic properties as the spontaneously formed unpatterned films. 


\section{Motivation}

Multiferroic and magnetoelectric materials have attracted a good deal of attention in recent years for their potential use in next generation electronic devices. ${ }^{1,2}$ For decades, the integrated circuit industry has been governed by Moore's Law and the International Technology Roadmap for Semiconductors for complementary metal-oxide semiconductor (CMOS) transistor logic. However, with current power densities and gate sizes approaching fundamental physical limits, Si-based CMOS logic architectures may be approaching the end of the roadmap. ${ }^{3}$ The field of spintronics offers one possible alternative to traditional CMOS logic and memory architectures. ${ }^{4,5}$ Spintronic logic and memory devices offer lower power consumption and non-volatility, which is not possible in CMOS architectures. ${ }^{3}$ In this section, a brief overview of multiferroic and magnetoelectric materials is presented, followed by a discussion of some spintronic device concepts, with a focus on the Reconfigurable Array of Magnetic Automata architecture, which motivated much of this thesis.

\section{Multiferroic and Magnetoelectric Materials}

Multiferroic materials are materials that simultaneously demonstrate multiple ferroic properties, such as ferromagnetism, ferroelectricity and piezoelectricity. Magnetoelectric material systems exhibit either electrical control of magnetic properties, or magnetic control of electrical properties. ${ }^{6}$ Magnetoelectric systems are closely related to multiferroic materials in that multiferroic materials often demonstrate magnetoelectric coupling due to the simultaneous presence of ferroelectricity and ferromagnetism. ${ }^{2,7}$ The ability to electrically control the magnetic moment of a material is a critical feature of most spintronic logic and memory systems. ${ }^{5,4}$ A Venn diagram outlining the relationship between various multiferroic properties and magnetoelectric behavior is shown in Figure 1-1. 


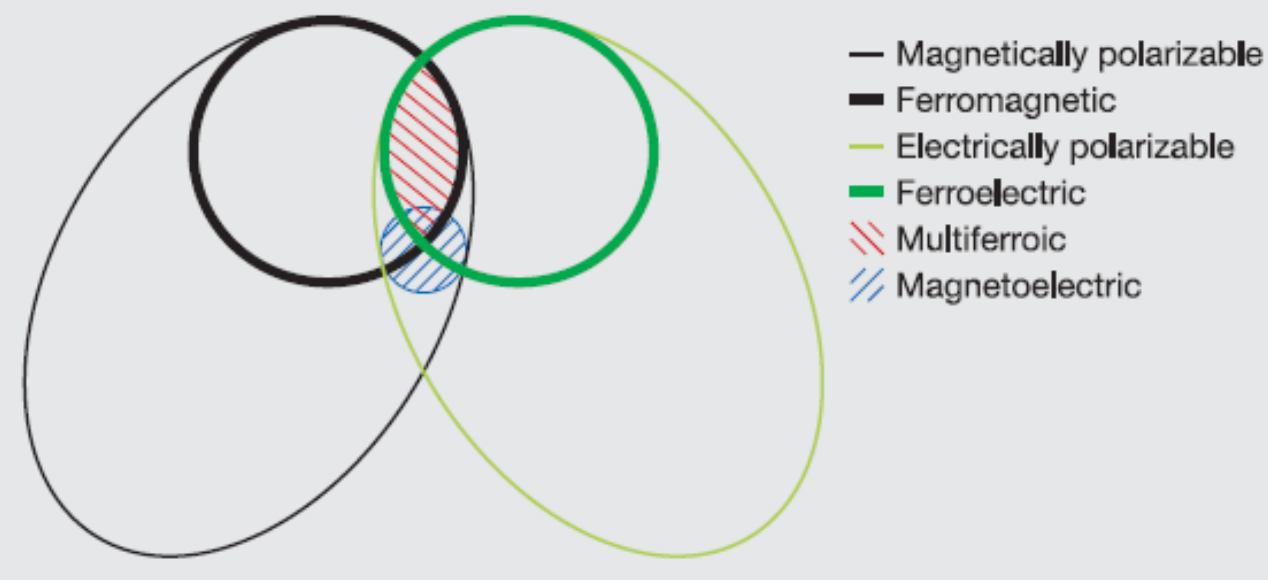

Figure 1-1. A Venn diagram showing the relationship of multiferroic materials to the class of materials systems that exhibit magnetoelectric properties. Adopted from Eerenstein, et al. ${ }^{1}$

In thin film form, multiferroic materials are commonly produced in three different ways. Single phase materials, such as the hexagonal manganites ${ }^{8,9}$ or strained $\mathrm{EuTiO}_{3}{ }^{10}$, can be grown epitaxially as single layer films which exhibit both ferroelectricity and ferromagnetism. Bilayer films, such as $\mathrm{La}_{1-}$

${ }_{x} \mathrm{Sr}_{\mathrm{x}} \mathrm{MnO}_{3} / \mathrm{BiFeO}_{3},{ }^{11}$ are designed so that one layer is ferromagnetic while another exhibits ferroelectricity. Through interfacial coupling effects, such as exchange bias, these bilayers can behave as magnetoelectric materials. Nanocomposite materials are another candidate system, with a ferromagnetic nanopillar embedded in a ferroelectric matrix. ${ }^{12,13}$ These materials become magnetoelectric through strain coupling at the interface between the pillar and matrix. ${ }^{14}$ Examples of the geometries for these three configurations are shown in Figure 1-2. 

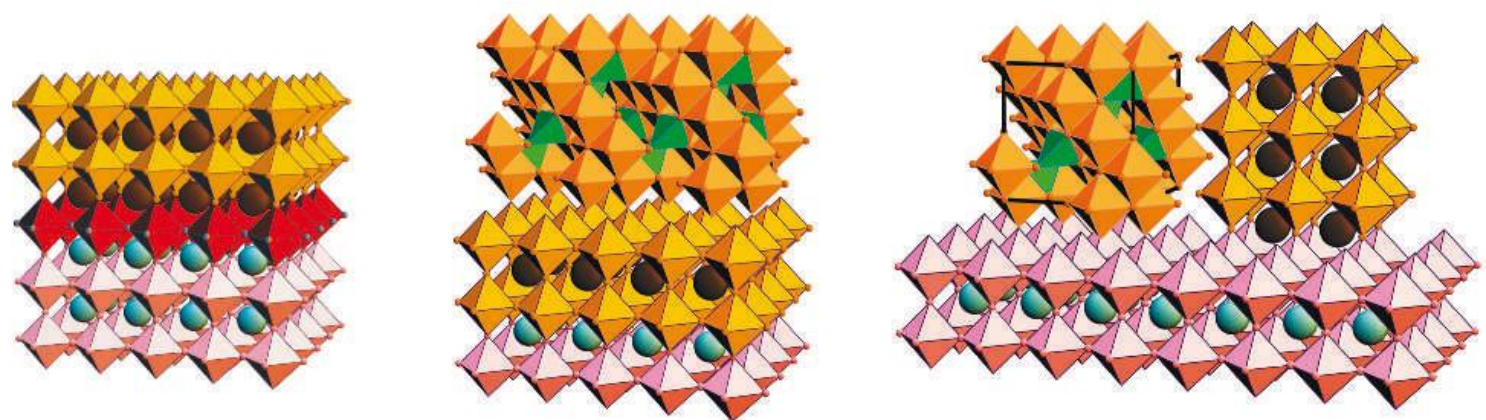

Figure 1-2. Types of multiferroic films: (left) A single phase film such as $\mathrm{EuTiO}_{3}$ or the hexagonal manganites; (center) A bilayer film where interfacial coupling through effects such as exchange bias produces multiferroic behavior; (right) A vertical composite such as the spinel-perovskite nanocomposites that produce multiferroic coupling through strain. Adopted from Ramesh and Spaldin. ${ }^{2}$

\section{Spintronics}

\section{Spintronic Memory Devices}

In modern computer systems, memory technology can be divided into two types of systems: randomaccess memory (RAM) and read-only memory (ROM). Static and dynamic RAM systems are used to store information currently in use by the computer processor, while ROM systems such as hard disk drives and flash memory store unused data. These two types of memory are needed because RAM systems are volatile, which means that they do not preserve information when the system is turned off. Similarly, ROM systems cannot be interfaced directly with the processor, because the energy needed to read and write bits of information, along with the time needed to do so, is too large to be practical during intense computations. Spintronic memory such as spin-transfer torque magnetic random access memory (STT-RAM) offers an intriguing alternative to existing memory technologies in that is both non-volatile and can be written and read quickly and efficiently enough to compete with existing RAM systems. ${ }^{3}$ In this way, STT-RAM represents an intriguing possibility for next-generation memory systems.

STT-RAM systems use a magnetic tunnel junction (MTJ) to store a bit of information. ${ }^{15}$ In an MTJ, a magnetic multilayer is grown with two distinct magnetic layers - a pinned layer that is magnetized along a single direction at all times and a free layer that may point parallel or anti-parallel to the pinned layer- 
separated by an insulating barrier which promotes quantum tunneling between the two materials. Because of the density of states in a ferromagnetic material is dependent on the spin of the electron in the material, the probability of tunneling across the barrier depends on the relative orientation of the two magnetic layers. ${ }^{16}$ If the magnetization of the layers is such that the moments point along the same direction (parallel), the tunneling magnetoresistance (TMR) is low and representing either a ' 0 ' or ' 1 '. Likewise, if the layers are magnetized in opposite directions (anti-parallel) the TMR is high and represents the opposite bit. By passing a small current through the MTJ, it is possible to read the bit. Through the use of spin-transfer torque, it is possible to rewrite the bit with new information by passing a current through the MTJ that is sufficient to switch the free magnetic layer. ${ }^{17}$ Certain complex oxides, such as the perovskite manganites, represent intriguing candidate materials for STT-RAM systems due to their high degree of spin polarization. ${ }^{18}$ Various groups have fabricated MTJs using epitaxial manganite multilayers and reported high TMR values at low temperature. ${ }^{19-21}$

\section{Spintronic Logic Systems}

Magnetic quantum cellular automata (MQCA) $)^{22}$ and nanomagnetic logic (NML) ${ }^{23}$ have been proposed as a possible approach to the creation of a magnetic logic alternative to complementary metaloxide-semiconductor (CMOS) transistor logic. Both systems share similar approaches to perform Boolean logic operations without using a transistor. An array of magnetic pillars or dots is fabricated lithographically such that neighboring dots are in close enough proximity for their dipole moments to couple anti-ferromagnetically, minimizing the overall magnetostatic energy of the system. By demonstrating a majority logic gate using metallic materials, researchers have developed a complete logic architecture that can perform the same logic operations as a CMOS logic system. ${ }^{24}$ Simulations have shown that NML or MQCA logic schemes could be lower power than the CMOS circuits they are intended to replace. ${ }^{25}$ However, an oscillating external magnetic field is required to 'clock' the system, making practical implementation difficult. 


\section{Reconfigurable Array of Magnetic Automata}

The need for an external magnetic clocking field is a significant impediment to implementation of the nanomagnetic logic systems discussed in the previous section. Fabrication of these systems is also problematic, as it is not trivial to perform the top-down lithography needed to produce the metallic islands in close enough proximity to achieve the dipolar coupling. To overcome these problems, an alternative approach has been proposed known as the reprogrammable array of magnetic automata (RAMA), which makes use of multiferroic materials that allow for electrical control of magnetic anisotropy. ${ }^{3}$ Other groups have proposed similar systems which employ ferroelectric materials for magnetoelectric control of a nanomagnetic logic system. ${ }^{26,27}$

RAMA uses a similar approach to logic operations as in NML, but uses multiferroic complex oxides instead of metals to create the magnetic pillars and introduces a matrix surrounding the pillars. In this approach, ferrimagnetic CFO pillars are fabricated and surrounded with BFO. The CFO pillars couple anti-ferromagnetically, as in NML, but do so with moments lying perpendicular to the plane of the array. The BFO matrix allows for magnetoelectric control of the pillars, which may allow the circuits to be reprogrammable. A uniform square array of pillars is patterned and then programmed by applying an electric field to the pillars that will not be a part of the circuit. This bias will change the ferroelectric state of the BFO matrix and strain the pillar, decoupling it from the MQCA circuit. As an alternative to the magnetic clocking field used in NML, it may also be possible to use the ferroelectric matrix as a means of electrically clocking the system for easier transmission of data in the logic circuit. A full schematic of the proposed design is shown in Figure 1-3. 

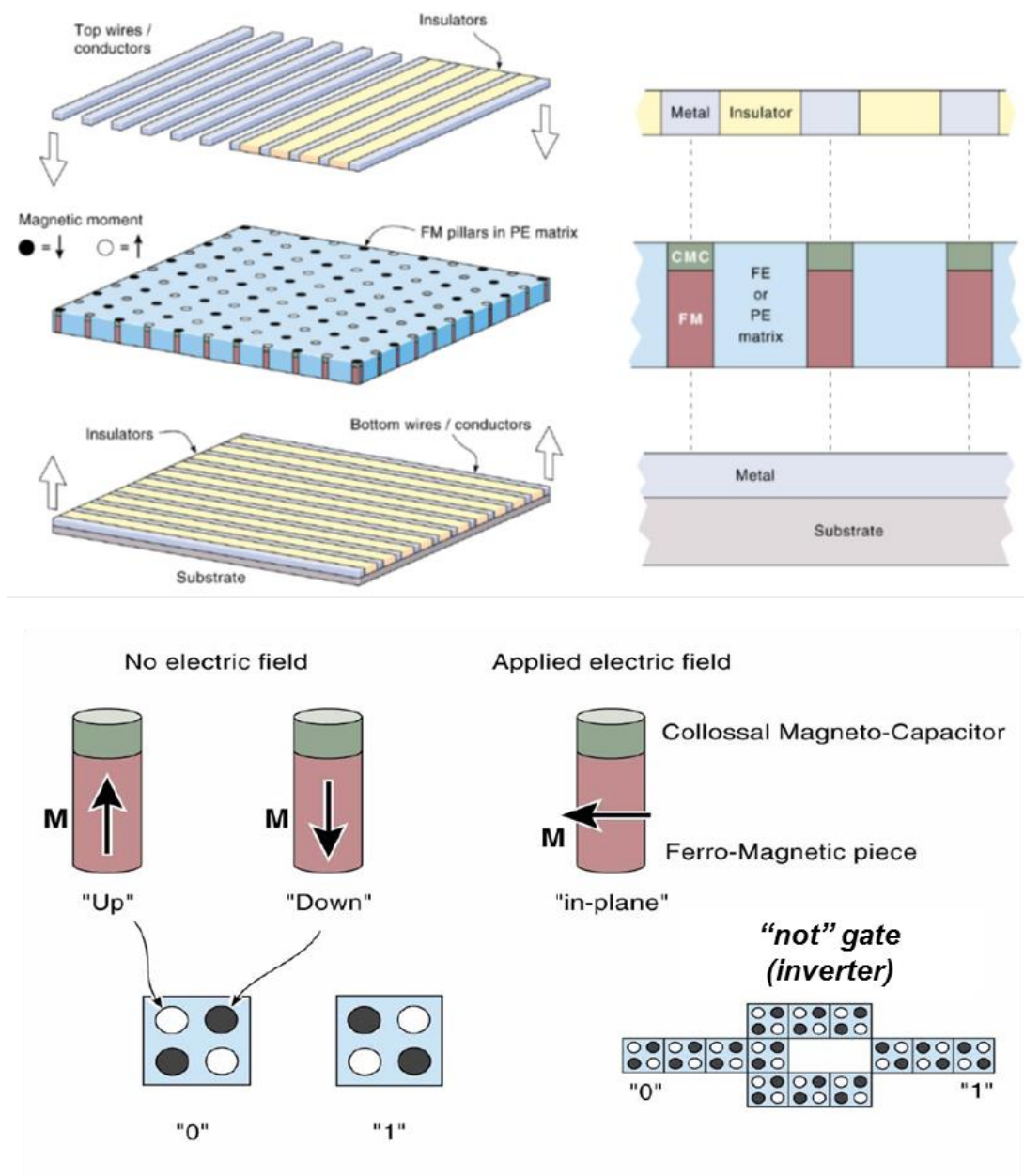

Figure 1-3. Schematic of Reprogrammable Array of Magnetic Automata (RAMA) logic architecture. A square array of CFO pillars with perpendicular magnetic anisotropy in a BFO matrix is produced. Individual pillars are addressed using wires above and below the array to write a ferroelectric domain in the BFO and create an MQCA logic circuit.

Micromagnetic simulations indicate that the RAMA architecture can perform the same types of logic operations as in the nanomagnetic logic schemes previously presented in the literature. ${ }^{28}$ It is found that for pillars with diameter of $20 \mathrm{~nm}$, a pitch (distance from center of one pillar to its neighbor) between 50 $\mathrm{nm}$ and $65 \mathrm{~nm}$ is sufficient for an error rate that becomes vanishingly small at room temperature. For larger pillar diameters, comparably larger pitches are needed. In the case of a $50 \mathrm{~nm}$ pillar, a pitch slightly greater than $100 \mathrm{~nm}$ is sufficient to produce dipolar coupling between the pillars. ${ }^{28}$ 


\section{Overview of Thesis}

The work performed in this thesis was motivated by the material needs to implement the Reconfigurable Array of Magnetic Automata (RAMA) presented in this chapter. A novel technique to direct the self-assembly of $\mathrm{CoFe}_{2} \mathrm{O}_{4}$ pillars in a $\mathrm{BiFeO}_{3}$ matrix is presented. Arrays of pillars with pitches as small as $100 \mathrm{~nm}$ have been fabricated using this technique. Unpatterned composites have also been fabricated and their magnetic and ferroelectric properties characterized and explained. Additionally, benchmarking of the novel pulsed electron-beam deposition technique has been performed and a variety of epitaxial growth modes have been demonstrated and explained for the first time in the literature. Chapter 2 presents the background of the materials systems of interest, including $\mathrm{BiFeO}_{3}, \mathrm{CoFe}_{2} \mathrm{O}_{4}$, epitaxial nanocomposites comprised of these materials, and discusses previous techniques to pattern the growth of oxide nanocomposites. Chapter 3 deals with the experimental procedures for the growth of epitaxial oxide films and the nanofabrication techniques employed to pattern these materials.

Characterization techniques, including as x-ray diffraction, electron microscopy, and scanning probe microscopy, are discussed in Chapter 4. In Chapter 5, experimental results for uniform thin films of $\mathrm{BiFeO}_{3}, \mathrm{CoFe}_{2} \mathrm{O}_{4}$, and $\mathrm{La}_{0.72} \mathrm{Sr}_{0.28} \mathrm{MnO}_{3}$ are presented, their magnetic and ferroelectric properties characterized, and the epitaxial growth modes discussed as a means of benchmarking the pulsed electronbeam deposition process. Unpatterned $\mathrm{BiFeO}_{3}-\mathrm{CoFe}_{2} \mathrm{O}_{4}$ nanocomposites are presented in Chapter 6 and their multiferroic properties are characterized and explained. In Chapter 7, experimental results demonstrating the directed self-assembly of $\mathrm{BiFeO}_{3}-\mathrm{CoFe}_{2} \mathrm{O}_{4}$ nanocomposites are presented. The microstructural and multiferroic properties of these materials are explained and compared to the unpatterned composites demonstrated. Chapter 8 summarizes the key findings and discusses possible directions for future work. 


\section{Background}

This research has focused on the epitaxial growth of complex oxide films and nanocomposites to enable next generation spintronic devices. In this chapter, I will discuss the science governing the growth of epitaxial thin films, which plays a critical role in understanding the structural properties of the films examined in this work. I will also discuss the properties of the materials examined, including $\mathrm{CoFe}_{2} \mathrm{O}_{4}$ and $\mathrm{BiFeO}_{3}$. Finally, previous results in the patterning and directed self-assembly of epitaxial films and nanocomposites will be presented.

\section{Epitaxial Thin Films}

Epitaxial film growth is a wide ranging field that encompasses all classes of materials, including metals, semiconductors, and oxides. The growth of epitaxial films is governed by surface chemistry, kinetics, and elastic properties of materials. There are two forms of epitaxial growth: homoepitaxy, which refers to the growth of a film consisting of the same material as the single crystal substrate, and heteroepitaxy, which is the growth of a different film material than the substrate. In this section, a brief overview of epitaxial growth modes and strain relaxation in heteroepitaxial films will be presented. The description will focus only on physical vapor deposition approaches, as chemical vapor deposition was not employed in this work.

\section{Growth Modes}

Epitaxial films can form in one of three different growth modes: layer-by-layer (Frank-van der Merwe) growth, ${ }^{29}$ island (Volmer-Weber) growth, ${ }^{30}$ and layer-plus-island (Stranski-Krastanov) growth. ${ }^{31}$ Layer-by-layer growth occurs when the deposited film uniformly wets the surface of the substrate, growing one atomic layer at a time. A special form of layer-by-layer growth is step-flow growth, whereby adatoms diffuse along the surface of the substrate until reaching a step edge, where the adatom then binds to the surface. ${ }^{32}$ In island growth, clusters of atoms nucleate on the surface of the substrate and form individual islands that are multiple atomic layers in height. ${ }^{33}$ In this case, the film does not wet the surface 
of the substrate, leaving some regions uncoated while the islands grow in height. As additional material is deposited, more islands nucleate and the islands eventually coalesce to produce a columnar epitaxial film. The special case of Stranski-Krastanov growth occurs when the initial interface energy between substrate and film promotes layer-by-layer growth. However, as the film increases in thickness, strain energy due to lattice mismatch between the film and substrate promotes island formation as a means of strain relaxation. ${ }^{33}$ A schematic of the three different growth modes is shown in Figure 2-1.
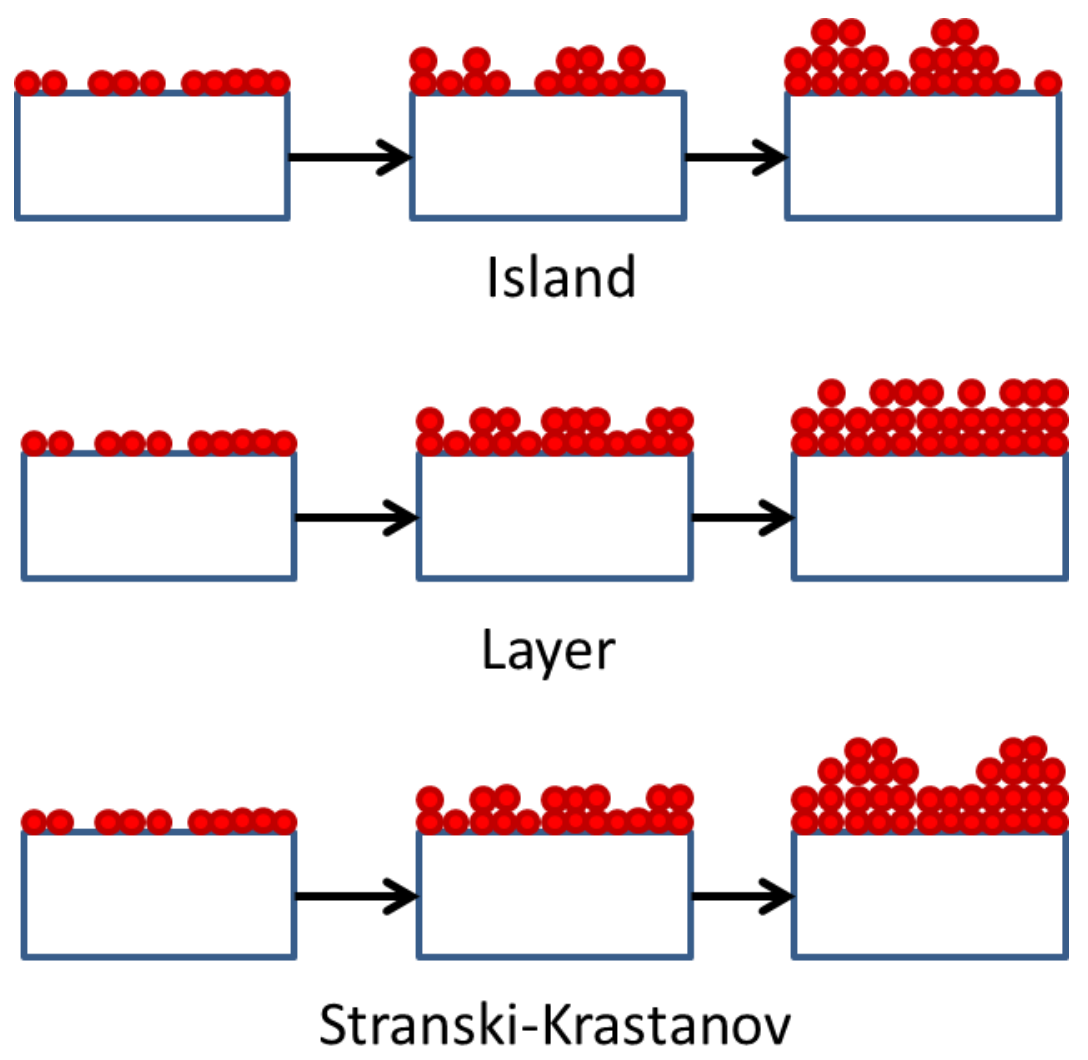

Figure 2-1. A schematic of the various growth modes possible during thin film growth.

Competition between nucleation in the formation of islands and crystal growth in the case of layerby-layer growth is a key governing feature in epitaxial film growth. The growth mode of a thin film is determined by the interfacial energy between the film and substrate, the surface energy of the film and the surface energy of the substrate. ${ }^{34}$ If one assumes that the atoms in the vapor are in equilibrium with those on the film surface it is possible to write the criteria for layer-by-layer growth as: 
$\Delta \gamma=\gamma_{F}+\gamma_{i}-\gamma_{S} \leq 0$

where $\gamma_{F}$ is the surface energy of the film, $\gamma_{i}$ is the interfacial energy between the film and substrate, and $\gamma_{S}$ is the substrate surface energy. The interfacial energy is governed by the chemical bonds between the substrate and film and the strain interactions due to lattice mismatch. In the case of island growth, the surface energy of the film or the interfacial energy between the materials is large enough that the film will not wet the surface and layer-by-layer growth is impossible. In this case, island nucleation occurs.

\section{Film Strain}

In heteroepitaxial growth with a film and substrate, strain is governed by the lattice mismatch between the two materials. The calculated lattice mismatch is given by:

$$
f=\frac{2\left(a_{f}-a_{s}\right)}{a_{f}+a_{s}} \sim \frac{a_{f}-a_{s}}{a_{f}}
$$

where $f$ is the dimensionless lattice mismatch, $a_{s}$ is the substrate lattice parameter, and $a_{f}$ is the bulk lattice parameter of the film. ${ }^{35}$ In the case of layer-by-layer growth, the film grows with an in-plane lattice parameter equal to the lattice parameter of the substrate. This is referred to as coherent strain and results

in a biaxial strain in the film. ${ }^{33}$ A schematic showing the strain in a heteroepitaxial film is shown in Figure 1. The strain energy, $E_{e}$, in the film is given by:

$$
E_{e}=Y d f^{2} /(1-v)
$$

where $Y$ is the Young's modulus of the film, $d$ is the film thickness, and $v$ is the Poisson's ratio of the film. For sufficiently small film thicknesses, $d$, the strain energy in the film is small enough to preserve coherent growth.

As film thickness increases, the elastic strain energy, $E_{e}$, increases linearly with the thickness. At a critical thickness, $d_{c}$, the strain energy becomes too large to preserve coherent epitaxial growth and misfit dislocations form to relax strain. ${ }^{33}$ The Matthews-Blakeslee critical thickness, ${ }^{36} d_{c}$, is given by the transcendental equation: 


$$
d_{c}=\frac{b}{8 \pi(1+v) f} \ln \left(\frac{\beta d_{c}}{b}\right)
$$

where $b$ is the burger's vector of the dislocation and $\beta$ is a constant between 0.701 and 2.72 , which is a proportionality constant for the radius of the strain field around the dislocation in units of $d_{c}$. A schematic showing the film strain due to lattice mismatch and the relaxation process through the formation of misfit dislocations is shown in Figure 2-2. Initially, dislocations form with a low density because the strain energy is insufficient to support sufficient dislocations to fully relax away all strain. As the thickness increases further, the dislocation density increases, relaxing strain and driving the lattice parameter of the film closer to the bulk value.

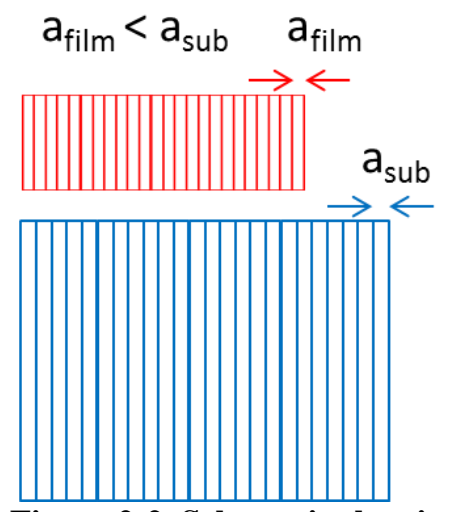

$$
\mathrm{d}<\mathrm{d}_{\mathrm{c}}
$$
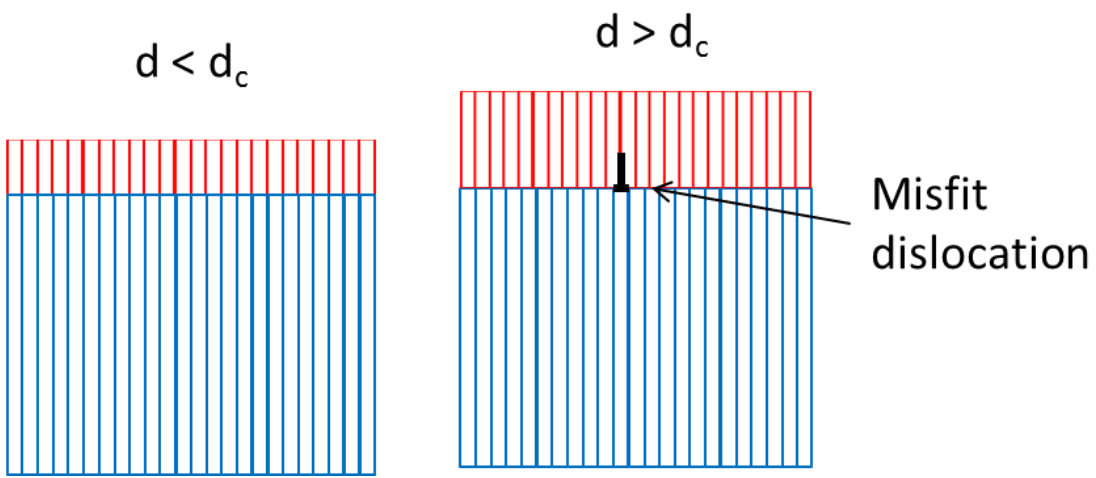

Figure 2-2. Schematic showing the growth of a heteroepitaxial film with strain due to lattice mismatch. (left) For thicknesses less than $d_{c}$ the film is coherently strained to the substrate in plane lattice. (right) For thicknesses greater than $d_{c}$ misfit dislocations form with a dislocation density that is thickness dependent.

\section{2. $\mathrm{CoFe}_{2} \mathrm{O}_{4}$}

$\mathrm{CoFe}_{2} \mathrm{O}_{4}(\mathrm{CFO})$ is a ferrimagnetic oxide with unusual magnetic properties that make it an ideal candidate for multiferroic and magnetoelectric devices. CFO has the cubic inverse spinel crystal structure, with $\mathrm{Co}^{2+}$ ions occupying half of the $\mathrm{B}$-site locations in the crystal, and $\mathrm{Fe}^{3+}$ ions at the remaining B-site locations and all A-site locations. ${ }^{37}$ This crystal structure corresponds to the space group $\mathrm{Fd} 3 \bar{m} .{ }^{38}$ Reports on the CFO lattice parameter vary slightly, but the most accepted value in the literature is $8.38 \AA^{39} .^{39}$ A rendering of the spinel CFO crystal structure is shown in Figure 2-3. 


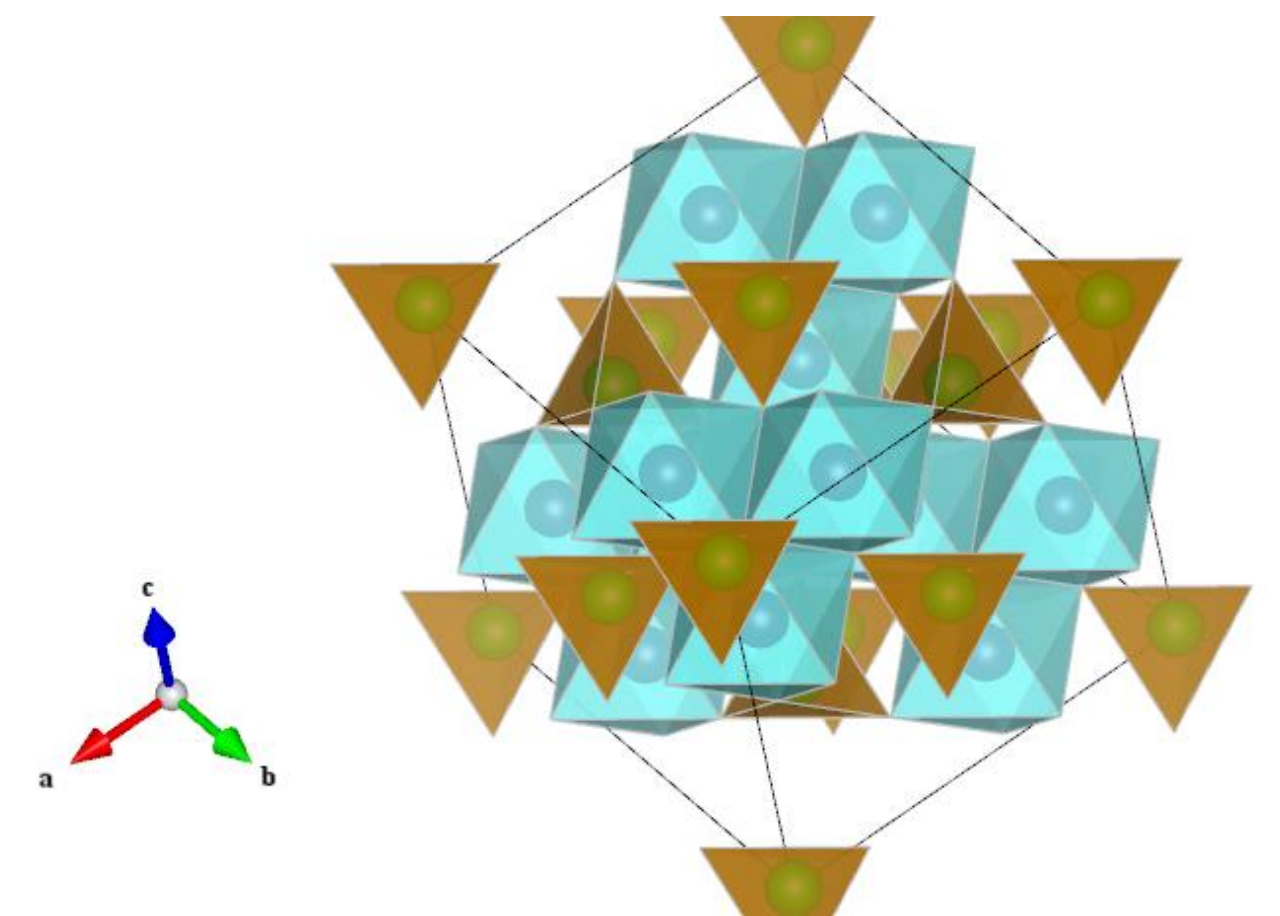

Figure 2-3. Rendering of a single unit cell of $\mathrm{CoFe}_{2} \mathrm{O}_{4}$ using VESTA. ${ }^{40} \mathrm{Fe}^{3+}$ ions occupy all tetrahedrally coordinated sites (brown) and half of the octahedrally coordinated sites (blue), while $\mathrm{Co}^{2+}$ ions occupy the remaining octahedral sites.

Due to the high degree of spin-orbit coupling in CFO, the magnetocrystalline anisotropy coefficient, $K_{l}$, has been reported to be $4 \times 10^{6} \mathrm{erg} / \mathrm{cm}^{3}$, while the magnetostriction coefficient, $\lambda_{100}$, can be as large as $5.9 \times 10^{-4} .41,42$ The negative value for $\lambda_{100}$ means that uniaxial compressive strain along a particular $<100>-$ type axis will induce uniaxial magnetic anisotropy in the same direction, making the axis of strain into the magnetic easy axis of the material. In the CFO magnetic lattice, the $\mathrm{Fe}^{3+}$ ions couple antiferromagnetically, producing no net magnetization, while the $\mathrm{Co}^{2+}$ ions couple ferromagnetically to give the material its magnetic moment. The saturation magnetization for CFO has been reported to be between 3.7 and $3.9 \mu_{\mathrm{B}} / \mathrm{Co} \mathrm{ion.}^{42}$

Much work has been done studying the effects of substrate choice on the magnetic properties of

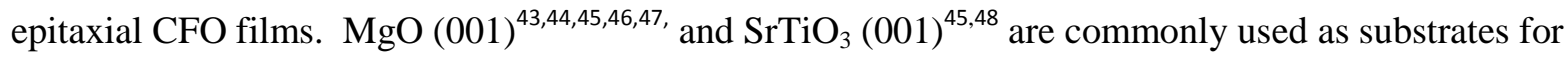
CFO films. $\mathrm{MgO}$ substrates share an excellent lattice match with the $\mathrm{CFO}$ film, as the $\mathrm{MgO}$ lattice parameter $(a=4.21 \AA)$ is almost exactly half of the CFO lattice parameter $(a=8.38 \AA) .{ }^{39}$ This 
commonly leads to $\mathrm{MgO}(002) \| \mathrm{CFO}(004)$ epitaxial correspondence and perpendicular magnetic anisotropy due to biaxial in-plane tensile strain in the film that results from the small lattice mismatch $(0.5 \%) .^{43,46,47}$ Films grown on $\mathrm{SrTiO}_{3}$ (STO) are also of interest because of its use as a substrate for many perovskite multiferroic materials. STO has a lattice parameter of $3.91 \AA$, which tends to seed films in the STO (002) || CFO (004) epitaxial configuration but leads to compressively strained films due to the lattice mismatch. ${ }^{45}$ It has also been shown that due to the lattice mismatch of $\sim 7 \%$ that CFO films will grow via the Volmer-Weber epitaxial island growth mode on STO. ${ }^{49}$

\section{Perovskite Oxides}

The perovskite class of complex oxides shares a similar $\mathrm{ABO}_{3}$ crystal structure shown in Figure 2-4. In the ideal cubic perovskite structure, A-site ions are located at the corners of a simple cubic lattice, Bsite ions are located at the body center site and $\mathrm{O}^{2-}$ ions are located on the face centered sites. The effect of this structure is to place the $\mathrm{B}$-site ion at the center of an octahedron of six $\mathrm{O}^{2-}$ ions. Different materials may have different crystal space groups due to various distortions of the oxygen octahedra that surround the B-site ion. ${ }^{50}$

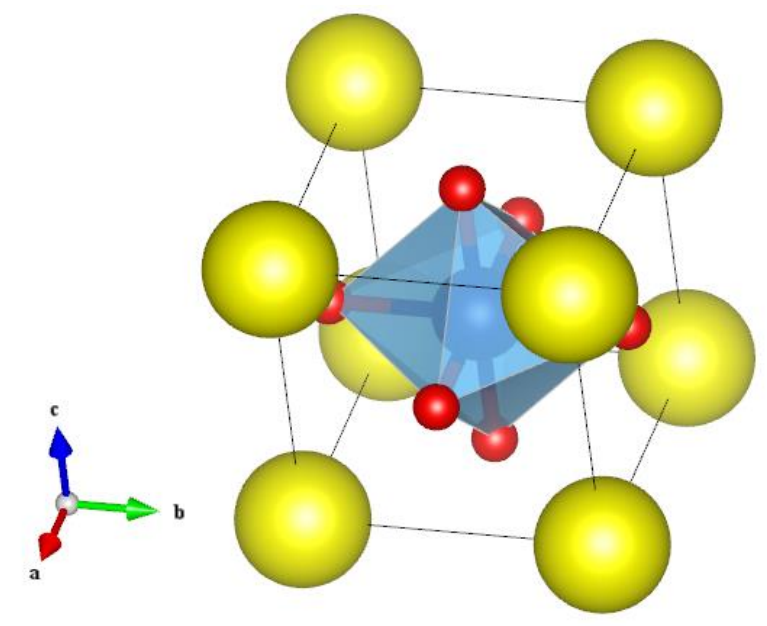

Figure 2-4. Rendering of perovskite unit cell using VESTA. ${ }^{40}$ Yellow ions represent A site ions, blue ions represent $\mathrm{B}$ site ions, and red ions are $\mathrm{O}^{2-}$ ions. The $\mathrm{O}^{2-}$ ions form an octahedron surrounding the $\mathrm{B}$ site ion. 


\section{4. $\mathrm{BiFeO}_{3}$}

$\mathrm{BiFeO}_{3}(\mathrm{BFO})$ is an anti-ferromagnetic, ferroelectric, making it a popular choice for many multiferroic applications. Bulk BFO has a rhombohedrally-distorted perovskite ${ }^{51}$ crystal structure, with a $\mathrm{Bi}^{3+}$ ion at the A-site and a $\mathrm{Fe}^{3+}$ ion at the $\mathrm{B}$-site, which produces an $R 3 c$ space group. ${ }^{52}$ The rhombohedral distortion is the result of a tilting of the oxygen octahedral cage which surrounds the central $\mathrm{Fe}^{3+}$. If the rhombohedral distortion is neglected, the unit cell can be represented with a pseudo-cubic lattice parameter of $3.965 \AA^{53}$

Ferroelectricity in the crystal is the result of the distortion of the ideal perovskite structure into the rhombohedral unit cell that is observed in BFO. A net ferroelectric polarization of $6.1 \mu \mathrm{C} / \mathrm{cm}^{2}$ along the $<111>$ axes was first observed in single crystal BFO in $1970 .{ }^{54} \mathrm{~A}$ commonly observed problem dating back to the first measurements has been the presence of leakage current when an electric field is applied to the material. It was not until 2003 that an epitaxial BFO film was demonstrated and the material became the focus of significant interest. ${ }^{55}$ In the seminal work of Wang et al., the authors grew epitaxial BFO films and found enhanced ferroelectric polarization of $55 \mu \mathrm{C} / \mathrm{cm}^{2} \cdot{ }^{55}$ Other measurements demonstrated that the value of the piezoelectric $d_{33}$ coefficient was between 40 and $90 \mathrm{pm} / \mathrm{V}$ depending on film thickness. Theoretical models have determined that the spontaneous polarization in BFO that induces ferroelectricity can primarily be attributed to the large Born effective charge on the $\mathrm{Bi}^{3+}$ ion. ${ }^{56}$ Switching of ferroelectric domains in BFO has also been shown to induce a transient strain in the film, which may be useful for magnetoelectric devices. ${ }^{57}$

Epitaxial BFO films are regularly grown on STO, which has a cubic perovskite crystal structure, similar to $\mathrm{BFO},{ }^{58}$ and on conductive $\mathrm{Nb}$-doped $\mathrm{SrTiO}_{3}{ }^{59,60}(\mathrm{Nb}: \mathrm{STO})$ when a bottom electrode is required for ferroelectric measurements. When the films are grown on STO, the BFO crystal changes from a rhombohedral unit cell to a distorted monoclinic unit cell with space group $\mathrm{Cm}$, due to the epitaxial strain at the substrate interface. ${ }^{55,61,62}$ Recent studies of BFO thin films grown with coherent epitaxial strain on $\mathrm{LaAlO}_{3}$ (LAO) substrates have demonstrated particularly interesting results. LAO is a rhombohedral 
perovskite with space group $R 3 \bar{c}^{63}$ and a pseudocubic lattice parameter of $3.79 \AA \AA^{64} \mathrm{BFO}$ films grown in the rhombohedral phase under coherent strain with LAO would be under a significant $-4.5 \%$ compressive strain, which would be expected to produce an out-of-plane expansion of the lattice parameter for a c/a ratio of approximately $1.07 .{ }^{65}$ Films grown under these conditions were found to exhibit a larger than expected out-of plane expansion, with c/a ratios of 1.23, suggesting that the BFO film had undergone a phase transition due to the epitaxial strain in the film. ${ }^{66}$ Theoretical models using density functional theory predicted that such a strain would induce a phase transition from the bulk rhombohedral phase $(R 3 m)$ to phase with a bulk tetragonal structure $(P 4 m m)$, which produces a film with a $C c$ space group. ${ }^{62}$ In the bulk phase, rotation of the oxygen octahedra in the unit cell is not present, which was confirmed in the thin film by synchrotron x-ray diffraction studies. ${ }^{62}$ Further experiments confirmed that the parent structure is in fact $\mathrm{P} 4 \mathrm{~mm}$ by growing Ba-doped BFO such that the film is fully lattice matched with LAO and does not distort to the $C c$ structure. ${ }^{67}$ Renderings showing the rhombohedral and tetragonal structures of BFO are shown in Figure 6. Very recent results have also shown that when a tensile in-plane strain is applied by growing $\mathrm{BFO}$ on a $\mathrm{NdScO}_{3}$ substrates (pseudocubic lattice parameter of $4.01 \AA$ ) a different strain induced phase transition occurs, producing an orthorhombic phase of indeterminate space group. ${ }^{68}$
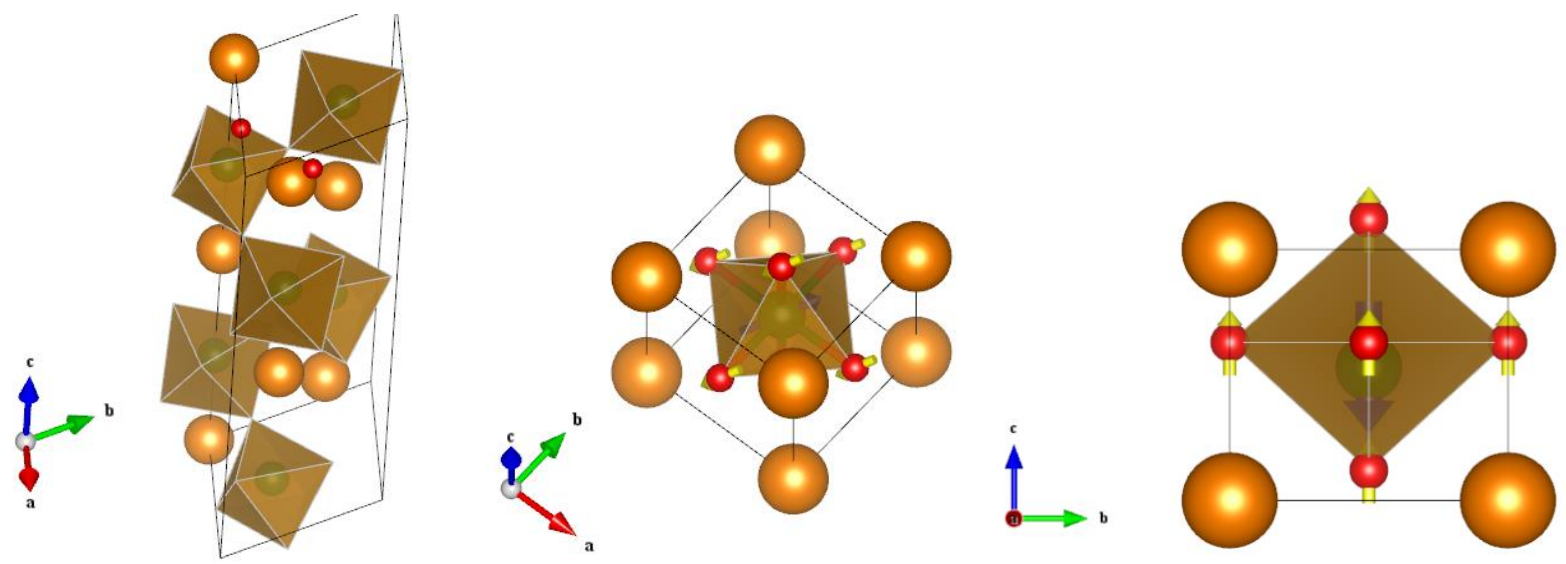

Figure 2-5. (left) The unit cell of unstrained $\mathrm{BiFeO}_{3}$ in the rhombohedral space group. (center) The pseudocubic unit cell of rhombohedral $\mathrm{BiFeO}_{3}$ showing the octahedral tilt pattern. (right) The tetragonal unit cell of $\mathrm{BiFeO}_{3}$ showing the ionic displacements in the material. All images rendered in VESTA. ${ }^{40}$ 
The so-called "morphotropic phase boundary" present in BFO is of particular interest for its potential to impart large strains by driving the system between the rhombohedral and tetragonal phases. When tetragonal-phase (T-phase) films are grown to thicknesses greater than $\sim 25 \mathrm{~nm}$ the strain begins to relax and regions of rhombohedral-phase (R-phase) BFO begin to develop with uniform stripes of epitaxial T and $\mathrm{R}$ phases present. ${ }^{65}$ Various works have shown that it is possible to electrically pole the system between this "stripe" state and the T phase. ${ }^{65,69,70}$ In particular, it has been shown that strains in excess of $1 \%$ can be induced in localized regions of the film through electrical poling. ${ }^{65}$

\section{Spinel-Perovskite Nanocomposites}

When mixed together to form a composite thin film, CFO and BFO have very interesting properties. It has been shown that when a ferroelectric perovskite oxide, such as BFO, is codeposited with a spinel oxide, such as CFO, the two materials will spontaneously phase segregate due to their immiscibility. ${ }^{13,71,72}$ This spontaneous phase segregation is the result of the different surface energies between the spinel and perovskite phases, where the $\{001\}$ surfaces of perovskites are the minimum energy surfaces, while the $\{111\}$ surfaces of spinels represent the minimum energy surface. ${ }^{73}$ The choice of substrate orientation thus affects the morphology of the resulting nanocomposite, since the perovskite phase will have the minimum surface energy on a (001) substrate, while the spinel phase will have the minimum energy on a (111) substrate. When grown on (001) substrates, which are commonly chosen to be STO, these composites segregate such that spinel pillars form in a perovskite matrix. ${ }^{73}$ The CFO pillars form faceted structures in BFO, with edges along the $<110>$-directions and pyramidal faces above the surface with $<111>$ facets to minimize the surface energy. ${ }^{73}$ A schematic of this structure is shown in Figure 2-6. 

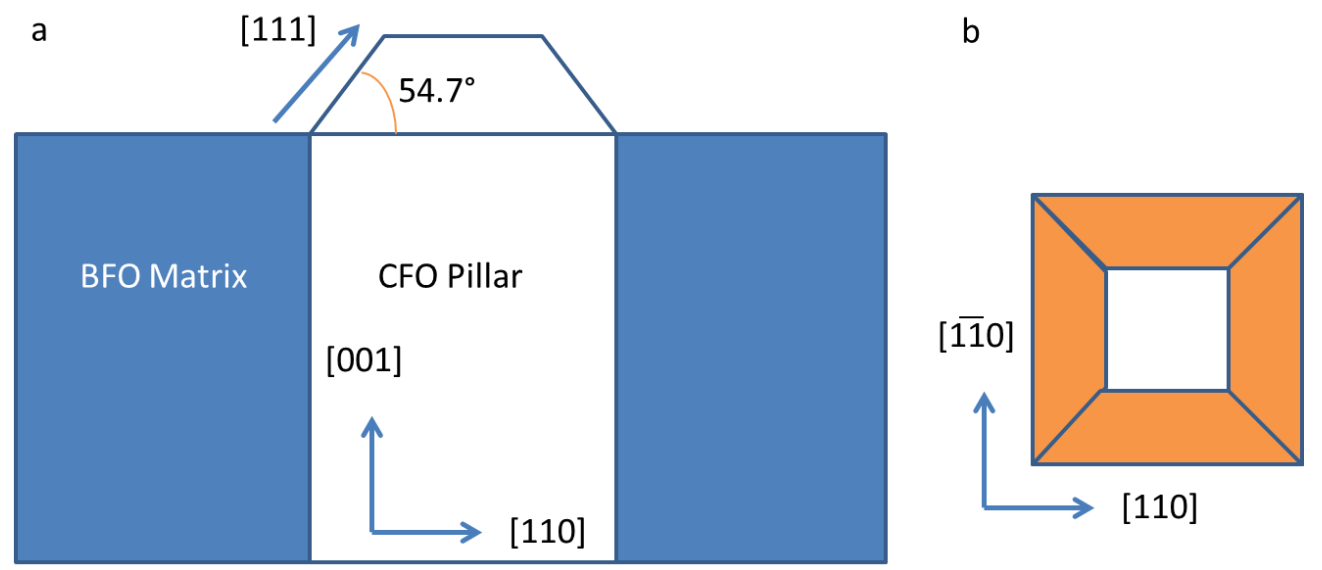

Figure 2-6. (a) Cross-sectional view of CFO pillar; (b) Top-down view of pillar.

Numerous works have shown that changing the ferroelectric polarization in the matrix induces a change in the magnetic anisotropy of the ferrimagnetic spinel pillar. ${ }^{7,57,74,75,76}$ A particularly intriguing result was presented by Zavaliche, et al, ${ }^{57}$ which showed that a change in the coercivity of the CFO nanopillars could be induced by selectively poling the BFO matrix via piezoresponse force microscopy (PFM). This result was attributed to transient strains induced in the CFO pillar during the ferroelectric switching process. However, work by others using $\mathrm{x}$-ray nanodiffraction has shown that it may be possible to induce a non-volatile strain in $\mathrm{Pb}(\mathrm{Zr}, \mathrm{Ti}) \mathrm{O}_{3}$ by poling the ferroelectric film via $\mathrm{PFM},{ }^{77}$ If such a non-volatile strain state were possible, it would represent an exciting prospect for use in the Reconfigurable Array of Magnetic Automata (RAMA) architecture. ${ }^{3}$

Some works have reported perpendicular magnetic anisotropy in CFO-BFO composites due to out-ofplane compressive strain in the CFO films. ${ }^{78,79,80}$ This strain is believed to arise from the vertical interface between the CFO pillar and the BFO matrix, which, if coherent, would yield a compressive strain. However, other works have shown that the interface between the two films is fully relaxed. ${ }^{72}$ Thus, the origin of the perpendicular anisotropy found in some composite films is not fully understood.

Spinel-perovskite nanocomposites have also been grown with a variety of other materials. Other ferroelectrics, such as $\mathrm{BaTiO}_{3},{ }^{13,75} \mathrm{PbTiO}_{3}{ }^{81,82}$ and $\mathrm{Pb}(\mathrm{Zr}, \mathrm{Ti}) \mathrm{O}_{3}{ }^{74,83-85}$ have been used with $\mathrm{CFO}$ pillars to 
produce composite magnetoelectric materials. Composites have also been grown with a tetragonal-phase BFO matrix through the use of an LAO substrate, which changes the magnetic anisotropy of the CFO pillar. ${ }^{86,87}$ An intriguing recent work demonstrated that by growing metallic $\mathrm{SrRuO}_{3}(\mathrm{SRO})$ as the matrix material with a CFO pillar, it is possible to optically control the magnetic anisotropy due to the photostrictive properties of SRO. ${ }^{88}$

\section{Patterned Complex Oxides and Nanocomposites}

Previous work in patterning multiferroic nanocomposites has been limited. One method to produce patterned magnetoelectric composites is to use a porous anodic aluminum oxide (AAO) film as a liftoff mask during deposition, which produces a hexagonal array pattern. ${ }^{89,84}$ In one approach, a $\mathrm{BaTiO}_{3^{-}}$ $\mathrm{CoFe}_{2} \mathrm{O}_{4}$ (BTO-CFO) multilayer is deposited onto the AAO film on an STO substrate, which yields a small amount of magnetoelectric response. ${ }^{89}$ Another technique is to use the AAO film to form CFO islands and then overcoat the islands with ferroelectric $\mathrm{Pb}(\mathrm{Zr}, \mathrm{Ti}) \mathrm{O}_{3}(\mathrm{PZT})$, which yields a composite that is both ferroelectric and ferromagnetic. ${ }^{84}$ Others have used a SiN membrane as a shadow mask to grow ferromagnetic $\mathrm{La}_{0.7} \mathrm{Sr}_{0.3} \mathrm{MnO}_{3}$ islands and overcoat them with ferroelectric $\mathrm{PbTiO}_{3}$, which produces an epitaxial composite structure with sub-micron dots and intriguing ferroelectric domain structures. ${ }^{90}$ However, none of these techniques offers the degree of magnetoelectric control found in the BFO-CFO 13 epitaxial nanocomposites..$^{80,57}$ Additionally, the AAO and membrane masks are not practical for the formation of a square array of pillars needed for the proposed memory and logic architectures. Another approach involves the use of block copolymers to order the formation of polycrystalline CFO pillars in a polycrystalline PZT matrix using a sol-gel process. ${ }^{91}$ This technique also produces a hexagonal array of pillars, but demonstrates a stronger magnetoelectric response than found in the other works. Larger, micron-scale polycrystalline $\mathrm{CFO}$ islands embedded in $\mathrm{PbTiO}_{3}$ matrix have been fabricated using ebeam lithography via a liftoff process, but such techniques are more difficult for smaller nanoscale islands 
and may not produce the epitaxial pillars and matrix that are desired. ${ }^{83} \mathrm{~A}$ bottom-up technique to arbitrarily define the location of individual pillars in the composite would be ideal for future technologies.

Limited work has been performed examining the formation of spinel islands on the surface of a substrate. Using an electron-beam lithography (EBL) liftoff process, one group patterned an array of pits in polymethyl methacrylate (PMMA) resist and spin coated CFO on an STO substrate using a sol-gel technique. $^{92}$ This process resulted in square arrays CFO islands of sizes ranging from 100 to $500 \mathrm{~nm}$ in diameter with pitch of $1 \mu \mathrm{m}$. After annealing, the authors found that the CFO islands facetted to form the same kind of dome structure that is seen in the epitaxial nanocomposites discussed above. A (001)oriented face sits on the top of the islands, with $\{111\}$-type facets rising from the substrate. Faceting of this type is also seen in CFO nanoparticles, which preferentially form $\{111\}$ facets. $^{93}$

Templated self-assembly is a popular technique to control the formation of a self-assembled structure. ${ }^{94,95}$ In this approach, the surface of a substrate is modified to constrain how a pattern will form during thin film deposition. Focused ion beam (FIB) patterning of a Si substrate to form pits has been shown to be effective in creating a preferred nucleation site for the formation of Ge epitaxial quantum dots. ${ }^{96,97}$ Similarly, EBL has been used to form SiC on the substrate surface, which acts as a nucleation site during Ge quantum dot growth. ${ }^{98}$ The approach chosen in this work was to define a CFO island on the surface of the substrate to act as a template for the self-assembly.

A similar technique was independently pursued by another group ${ }^{99}$ and was recently published after the initial results in this thesis. ${ }^{100}$ In this work, the group fabricated $(\mathrm{Fe}, \mathrm{Zn})_{3} \mathrm{O}_{4}$ nanoscale pads with lateral dimensions ranging from $100 \mathrm{~nm}$ to $3 \mu \mathrm{m}$ and deposited a BFO film on top of the template substrate. They found that BFO adatoms preferentially diffuse off of the pads if the diffusion length is sufficient for the adatoms to reach the edge of the pad. This phenomenon is a result of the large interface energy between the spinel and perovskite phases, which is the same physics that governs the growth of the epitaxial nanocomposites. Following the demonstration of directed self-assembly in this work, the same 
group also demonstrated the patterning of Fe pillars in a matrix of $\mathrm{LaSrFeO}_{4}$ using a similar technique to what was presented here. ${ }^{101,102}$ 


\section{Thin Film Growth and Nanofabrication}

\section{Epitaxial Thin Film Growth}

\section{Introduction}

Ferroelectric, multiferroic and correlated oxides have traditionally been grown by several different techniques, including chemical vapor deposition (CVD), sputtering, pulsed laser deposition (PLD) and molecular beam epitaxy (MBE). ${ }^{35}$ Detailed discussion of films grown by CVD and sputtering is beyond the scope of this work, though high quality epitaxial films can be grown using both techniques. ${ }^{103} \mathrm{MBE}$ became a popular technique for the layer-by-layer growth of complex oxides with the increase in research in the high temperature superconductors and is now in wide use for the growth of magnetic and correlated oxide films as well. ${ }^{104,105}$ MBE techniques, such as reflection high energy electron diffraction (RHEED), ${ }^{106}$ have been employed to grow multilayer and superlattice films and examine interfacial phenomena that depend on precise, atomic-layer control of film thickness and surface termination. ${ }^{107}$

PLD is a popular laser ablation approach to the growth of complex oxide films due its unique ability to grow a stoichiometric film. ${ }^{33}$ This capability makes it ideal for complex oxides, where maintaining the stoichiometry of the various cation elements within the crystal is critical. In recent years, MBE approaches to film growth have been applied to PLD, which has led to the term "laser MBE" to describe films grown via PLD with in situ RHEED apparatus to monitor film epitaxy. ${ }^{108,109}$ It is now widely accepted that films, multilayers and superlattices grown using the laser MBE technique may have crystal and interface quality comparable to those grown via traditional MBE. ${ }^{35}$ However, PLD may be inconvenient because of the need for a large, expensive excimer laser and an optics setup to focus the laser beam into the deposition chamber, and the toxic gases.

A relatively new technique, pulsed electron-beam deposition (PED), offers an intriguing alternative to PLD. Instead of a laser source to ablate the target, a high current, high voltage electron pulse is employed. 
This allows for a self-contained deposition system which allows multiple sources to deposit simultaneously. The PED technique uses a channel spark mechanism to produce energetic electron pulses. ${ }^{110}$ When directed at a target these pulses penetrate the surface material and cause rapid evaporation due to non-equilibrium heating. The pulse duration is $\sim 100 \mathrm{~ns}$ with energies on the order of a Joule. PED has many similarities to PLD, such as average pulse duration, energy and non-equilibrium ablation of target materials. Table 3-1 lists the characteristic properties of Pulsed Electron Beam Sources. Due to the smaller absorption depth of electrons as compared to UV photons, PED is capable of depositing wide band gap materials, semiconductors, highly reflective metals, and organic materials.

Table 3-1. Pulsed Electron Beam Sources parameters. ${ }^{110}$

\begin{tabular}{|c|c|}
\hline Maximum discharge voltage & $20 \mathrm{kV}$ \\
\hline Electrical efficiency & $30 \%$ \\
\hline Stored Energy & $3 \mathrm{~J}$ \\
\hline Gas pressure & $4-30 \mathrm{mTorr}$ \\
\hline Discharge time & $\sim 80-100 \mathrm{~ns}$ \\
\hline Repetition rate & $1-100 \mathrm{~Hz}$ \\
\hline Electron energy in the beam & $\leq 15 \mathrm{keV}$ \\
\hline Electron current & $1.5 \mathrm{kA}$ \\
\hline Electron beam diameter at the target & $\sim 2-3 \mathrm{~mm}$ \\
\hline Beam Current density & $\leq 10^{5} \mathrm{~A} / \mathrm{cm}^{2}$ \\
\hline Power in the beam & $\leq 15 \mathrm{MW}$ \\
\hline Power density in the beam & $\leq 500 \mathrm{MW} / \mathrm{cm}^{2}$ \\
\hline Maximum Range of electrons & $0.4 \mu \mathrm{m}$ \\
\hline
\end{tabular}

The technique, first referred to as "pseudospark ablation", was initially demonstrated in 1990 via the growth of a $\mathrm{YBaCuO}(\mathrm{YBCO})$ film. ${ }^{111}$ Since that time, PED technology has progressed and the conditions required for optimized growth have been studied. ${ }^{112}$ To date, many of the oxide films grown via PED have been high temperature superconductors, such as $\mathrm{YBCO}^{113-115}$ and $(\mathrm{La}, \mathrm{Sr})_{2} \mathrm{CuO}_{4} \cdot{ }^{116-118}$ One group also grew the correlated magnetic oxide $\mathrm{La}_{0.6} \mathrm{Pd}_{0.4} \mathrm{Mn}_{0.8} \mathrm{Ru}_{0.2} \mathrm{O}_{3}$ using the technique. ${ }^{119}$ In most of these cases, however, the films grown were deposited at high growth rates, leading to either textured films or thick epitaxial films with rough surfaces and particulate on the surface. For films grown via this technique to be comparable to those grown via conventional or laser MBE, reduced growth rates and particulate-free 
films are needed. In particular, optimal film growth conditions typically occur with thermalized adatoms, which have velocities governed by their thermal energy rather than the energy imparted during ablation. ${ }^{120}$

\section{Pulsed Electron Deposition Procedures}

In this thesis, all complex oxide thin films are grown using a Neocera Pulsed Electron-Beam Deposition (PED) system. PED is a relatively new technology for thin-film deposition, but has been shown to produce high quality complex oxide thin films. ${ }^{120.112,121}$ PED is somewhat similar to the more common pulsed laser deposition (PLD) technique, but differs in some key ways. Most notably, the optimal deposition rate for PED is roughly four times lower than that for PLD to deposit material. ${ }^{112}$ The growth conditions for samples in this work, such as substrate temperature, gun voltage, and operating pressure and gas mixture, were varied depending on the material being deposited and the desired film morphology. The appendix to this thesis provides a chart with a detailed list of the growth conditions for each sample shown in this work.

A view inside the system vacuum chamber is shown in Figure 3-1. The electron guns are mounted to the side of the chamber and electron pulses from each gun are directed to the target surface via an alumina tube mounted inside the end of the electron gun. Two different configurations of the gun were used during this work, as the system design was evolved by the manufacturer over the time. Initial films were grown with an alumina tube protruding from the electron gun held in place by a cooling water coil, which kept the tubes from overheating. This configuration can be seen in Figure 3-1(A) on the right electron gun. The updated design incorporates a metallic sleeve that encases the tube and is believed to improve the electrostatic conditions to prevent electrical discharge to the cooling water coil. This configuration is shown on the left gun in Figure 3-1(A). An alumina cap is mounted to the end of the sleeve to prevent any shorting between the sleeve and the tube. Examples of the sleeve and tube are shown in Figure 3-2. 


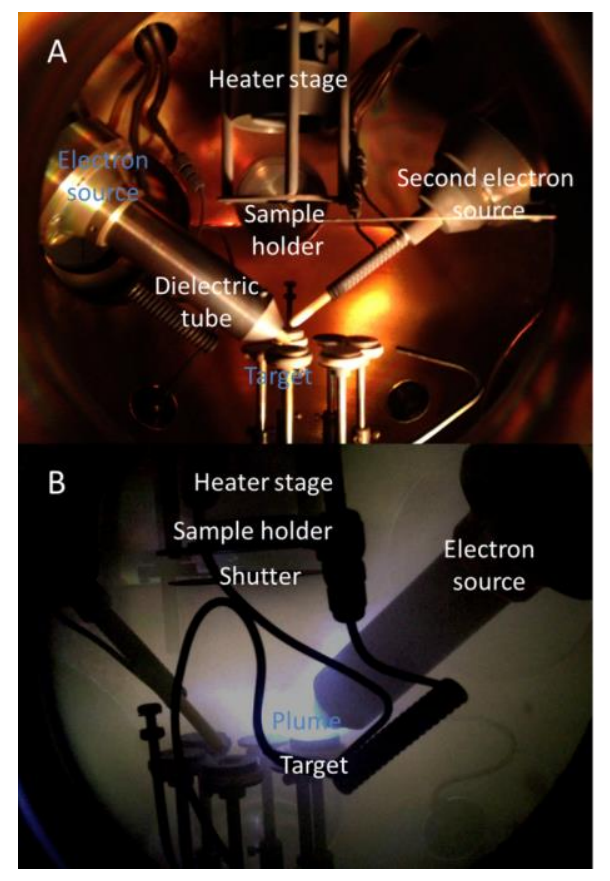

Figure 3-1. Photographs of the PED system. (A) A view inside the chamber showing the left electron gun with the new metallic sleeve and the right electron gun with the older cooling coil holder, the substrate holder and heater, and the target stage. (B) An image of the plume produced during ablation of the $\mathrm{CoFe}_{2} \mathrm{O}_{4}$ target.

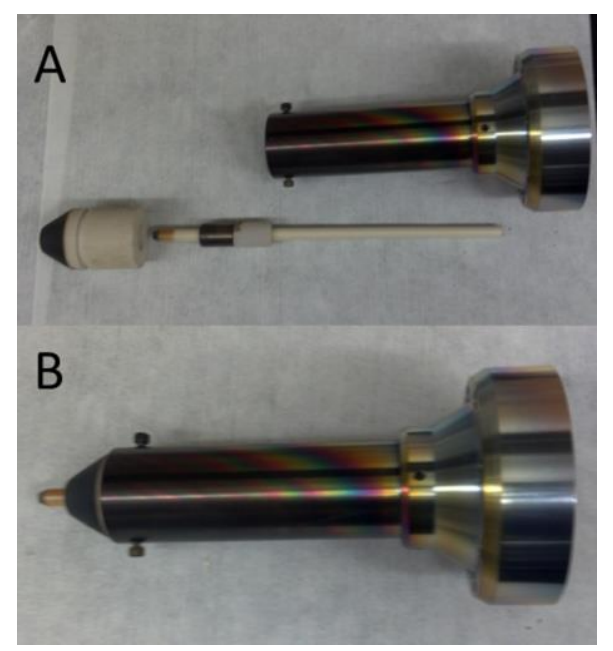

Figure 3-2. (A) Disassembled sleeve (top), tube (bottom) and alumina cap (left). (B) Assembled system.

The two materials that are the primary focus of this research are CFO and BFO. Additional

$\mathrm{La}_{0.72} \mathrm{Sr}_{0.28} \mathrm{MnO}_{3}$ (LSMO) films have been grown to demonstrate the epitaxial growth modes possible in films grown via PED and for use as a bottom electrode for ferroelectric measurements. In the case of 
CFO, a stoichiometric $\mathrm{CoFe}_{2} \mathrm{O}_{4}$ target is used to synthesize films with the same composition. A stoichiometric LSMO target was also used for those films. For BFO two different targets have been used. Films were grown using a $\mathrm{Bi}_{1.15} \mathrm{FeO}_{3}$ target and a $\mathrm{Bi}_{1.2} \mathrm{FeO}_{3}$ target. The additional $\mathrm{Bi}$ is required due to the high volatility of $\mathrm{Bi}$ at elevated temperatures, which leads to the loss of $\mathrm{Bi}$ from the films. ${ }^{122}$ Ceramic targets (diameter $=1$ inch) used in this work were fabricated by Kurt J. Lesker Inc. The targets are placed in an alumina dish and held on the carousel stage beneath the electron gun. A representative target and alumina dish are shown in Figure 3-3. To ensure uniform ablation of the targets, the carousel rasters the target about its center and the target itself rotates beneath the electron gun. This technique allows for ablation of the entire target surface during the deposition process, and minimizes the formation of any "racetrack" grooves. For this work, the targets were rotated at a rate of $20^{\circ} / \mathrm{sec}$ and rastered at a rate of $2 \% \mathrm{sec}$. The rotation rate produces a rotational period of 13 seconds to complete $360^{\circ}$, while the raster rate produces a period of 15-20 sec to traverse the entire target (the target surface covers an angle of approximately $20^{\circ}$ out of the carousel's circumference).

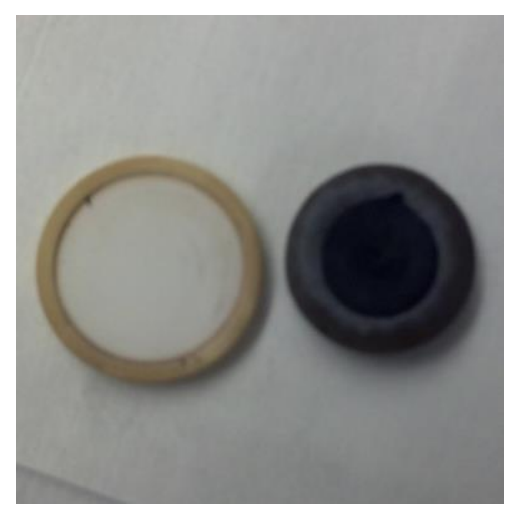

Figure 3-3. Alumina holder (left) and ablated Bi1.2FeO3 target (right). Target is 1 inch in diameter.

A load-lock chamber is attached to the main chamber to quickly transfer samples without breaking the vacuum in the main/growth chamber. This chamber also uses a turbo-molecular pump manufactured by Pfeiffer Vacuum Inc. Samples are mounted on an inconel holder using commercially available Fast Drying Silver Paint (Ted Pella Inc.). The use of different varieties of silver paste is believed to change the actual substrate temperature slightly and changes the growth conditions, so it is important that only the 
Fast Drying Silver Paint is used. An example of three mounted substrates is shown in Figure 3-4. The sample holder is placed within a transfer collar that sits on the heater stage within the chamber. The stage heater is lowered into close proximity with the sample holder, but not placed in direct contact so as to allow the sample holder to rotate for uniform deposition. The sample holder is rotated at an angular velocity of $120 \% \mathrm{sec}$. The presence of the space between the sample and heater means that the temperature setting used within the computer control program is not equal to the substrate surface temperature.

Previous calibrations were performed using a thermocouple mounted to a sample holder to determine the relationship between the set temperature and the temperature on the surface of the sample holder. ${ }^{123}$ This graph is shown in Figure 3-5. These calibrated temperatures should be treated as estimates, given that the system is not equipped with a pyrometer or other system to directly measure the substrate temperature. In previous works, an uncertainty in the substrate temperature of $25^{\circ} \mathrm{C}$ has been assumed. ${ }^{100}$

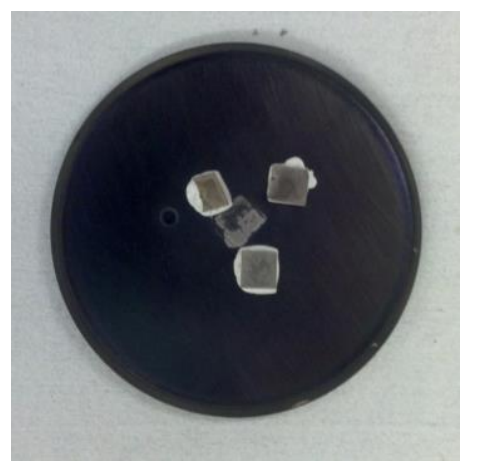

Figure 3-4. Sample plate with substrates mounted using fast drying silver paint.

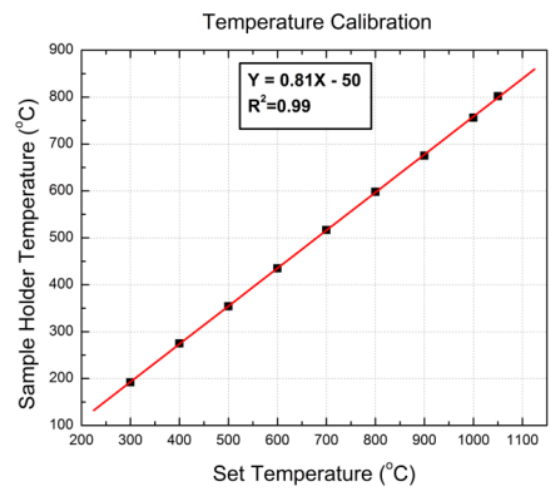

Figure 3-5. Temperature calibration data showing substrate temperature as a function of setpoint temperature. ${ }^{123}$ 


\section{Substrate Preparation}

Substrates in this study were prepared in different ways depending on the desired surface properties. For samples that did not require a specific surface termination, substrates were cleaned via sonication in acetone and isopropanol before use, but not treated with any etching or annealing process. The "asreceived" substrates (Crystec GMBH) show minimal roughness and exhibit step-edges, making treatments unnecessary if single surface terminations are not needed for a sample. Representative atomic force microscopy (AFM) surface topography scans are shown in Figure 3-6.

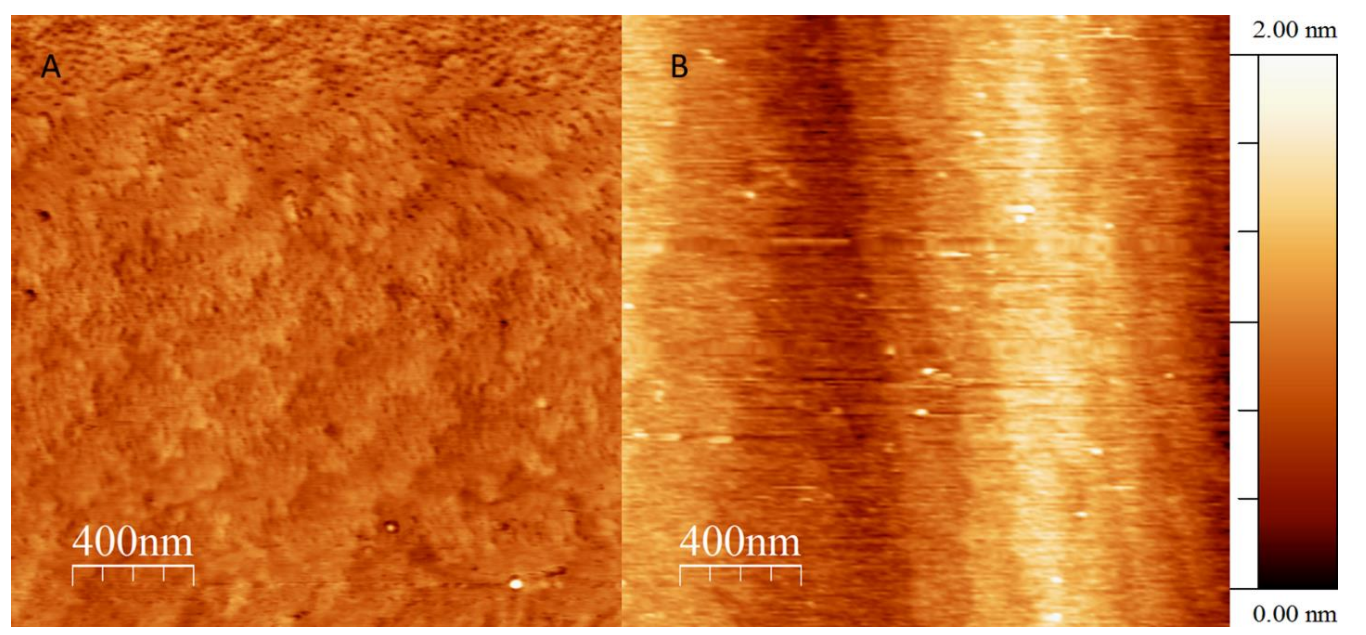

Figure 3-6. As received SrTiO3 (A) and LaAlO3 (B) substrates showing multiple terminations and minimal surface roughness with visible step edges. Note the presence of twinning in the LaAlO3 substrate due to the rhombohedral room temperature crystal structure.

To prepare $\mathrm{TiO}_{2}$-terminated surfaces on $\mathrm{SrTiO}_{3}(\mathrm{STO})$ and Nb-doped $\mathrm{SrTiO}_{3}(\mathrm{Nb}: \mathrm{STO})$ substrates, a commonly used etching and annealing technique was employed. ${ }^{58}$ Substrates were initially cleaned using an ultrasonic agitator for 5 minutes each in acetone and isopropanol baths, followed by 10 minutes in deionized water. The substrates were then etched for 30 seconds in a bath of commercially available buffered oxide etchant (Sigma-Aldrich) containing hydrofluoric acid. The samples were then rinsed in deionized water for at least 1 minute and annealed in an open air tube furnace for 1 hour at $1100{ }^{\circ} \mathrm{C}$. This procedure produced atomically smooth substrates with regular step-edges for STO and atomically smooth 
substrates with jagged step-edges that were generally multiple unit cells in height for Nb:STO.

Representative AFM scans of both surfaces are shown in Figure 3-7.
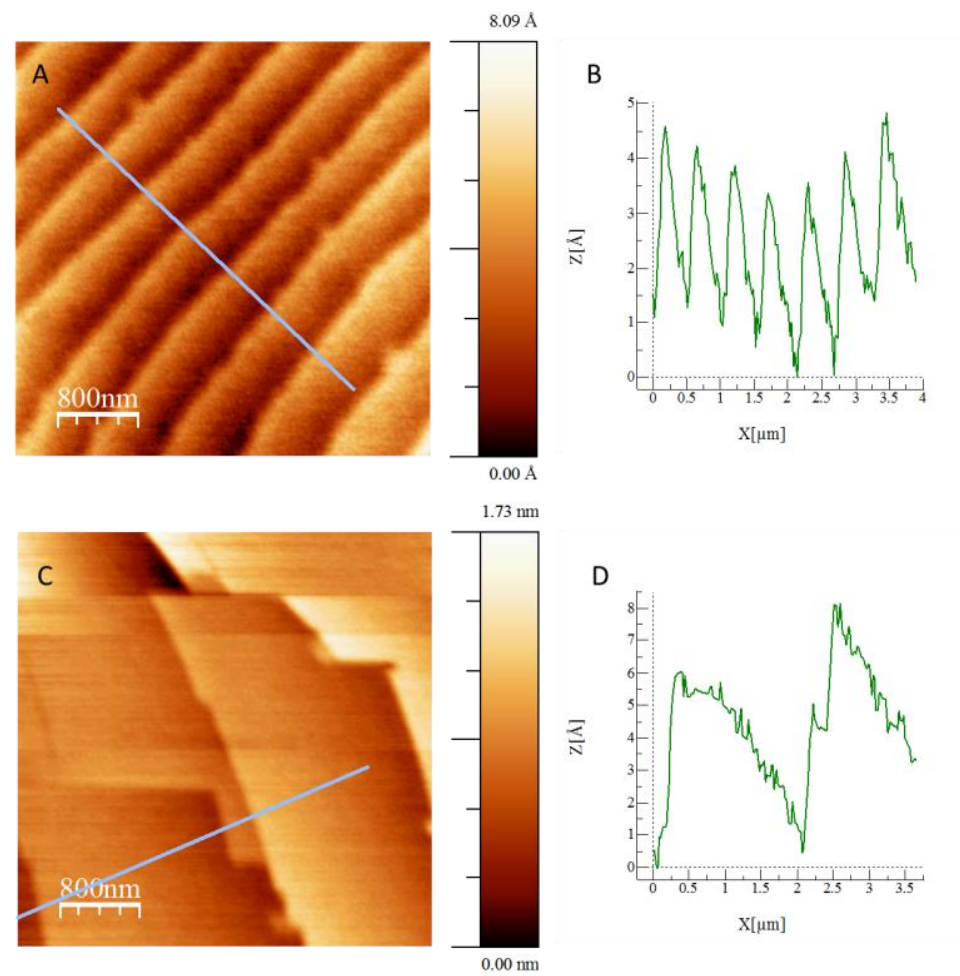

Figure 3-7. A) AFM topography scan for representative $\mathrm{SrTiO}_{3}$ substrate; $\mathrm{B}$ ) Line profile along gray line in (A); C) AFM topography scan for representative $\mathrm{Nb}$-doped $\mathrm{SrTiO}_{3}$ substrate; D) Line profile along gray line in $(\mathrm{C})$.

$\mathrm{MgO}(001)$ substrates were prepared to have atomically-smooth surfaces for growth of $\mathrm{CoFe}_{2} \mathrm{O}_{4}$. The substrates were sonicated in acetone and isopropanol baths and annealed at $1200^{\circ} \mathrm{C}$ for 1 hour in a tube furnace. The resulting surface had wavy step-edges and sparse surface islands and pits due to internal contaminants diffusing to the surface, but was otherwise smooth. ${ }^{124}$

\section{Nanoscale Fabrication Techniques}

To direct the self-assembly of CFO-BFO epitaxial nanocomposites, nanoscale fabrication in a cleanroom environment was performed to pattern small CFO islands on various substrates. All fabrication work presented here was performed at the National Institute of Standards and Technology 
Center for Nanoscale Science and Technology in Gaithersburg, Maryland. In this section, the procedures for amorphous Si sputtering, electron-beam lithography (EBL), and reactive ion etching (RIE) of the patterned samples are described. A schematic outlining the entire fabrication process is shown in Figure $3-8$.

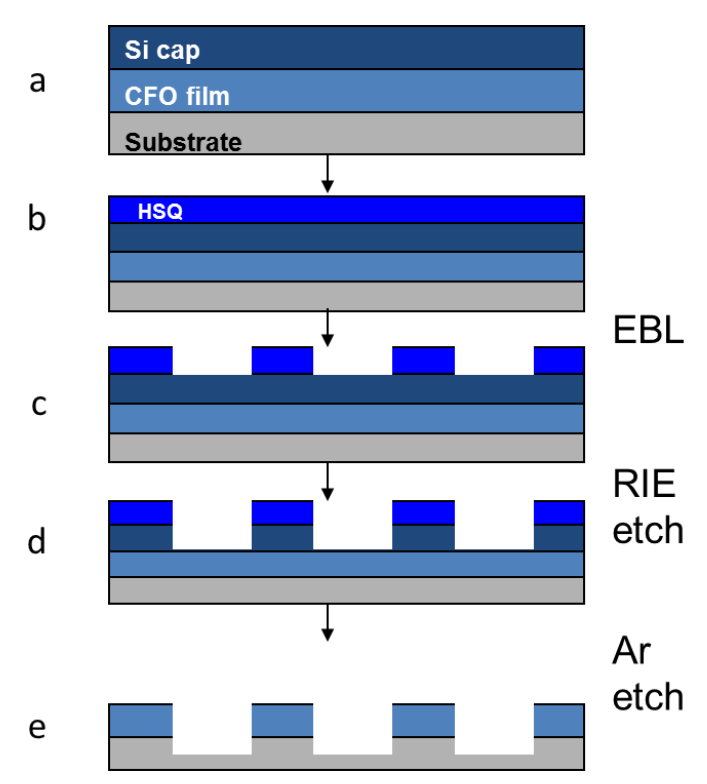

Figure 3-8. (a) Initial structure before patterning; (b) HSQ resist spun onto Si cap; (c) Resist is patterned to form dots using e-beam lithography; (d) Pattern is transferred through the Si cap using RIE; (e) Pattern is transferred through CFO film using Ar mill. The STO substrate is partially etched during this process.

\section{Silicon Sputtering}

The Si capping layer was grown at room temperature using an RF sputter system in the NIST Center for Nanoscale Science and Technology (CNST) NanoFab. The system has a base pressure of better than $3 \times 10^{-6} \mathrm{~T}$. An Ar atmosphere of 3.7 mTorr was used, with $400 \mathrm{~W}$ RF power applied to an undoped $\mathrm{Si}$ sputter target. This produces a deposition rate of $1.1 \AA$ A/sec based on recipes provided by NIST staff. In practice, the deposition rate varies by $\sim 10 \%$ from run to run due to long term drift in the equipment between uses. Deposition times were 180 seconds to produce films with calibrated thickness of approximately $20 \mathrm{~nm}$. The Si film is expected to be amorphous and produces what appears to be a conformal coating of the CFO islands. ${ }^{125}$ 


\section{E-beam Lithography Patterning}

Dow Corning XR-1541 hydrogen silsequioxane (HSQ) negative tone e-beam resist was used for patterning. The resist was diluted in a 1:1 mixture with methyl isobutyl ketone (MIBK) to form a 3\% solids content HSQ solution. HSQ is currently the most used negative tone resist available for EBL and has been used to produce 10-nm nanopillar arrays with pitches (center-to-center distance) as small as 25 nm. ${ }^{126,127}$ However, HSQ has been shown to adhere more readily to Si than to other materials, which necessitates the Si capping layer on top of the CFO film. ${ }^{125}$ The resist was spin coated on the sample at $3000 \mathrm{rpm}$ for 35 seconds to produce a resist thickness of between 60 and $70 \mathrm{~nm}$, as measured by optical ellipsometry.

Electron beam lithography (EBL) was performed using a Vistec VB300 EBL and JEOL JBX 6300-FS systems at the CNST NanoFab with area exposure doses of $5-30 \mathrm{mC} / \mathrm{cm}^{2}$ with an accelerating voltage of $100 \mathrm{kV}$. The EBL pattern was designed so that a variety of pillar shapes, widths and pitch sizes would be patterned onto the same sample. Alignment marks surrounding a $100 \mu \mathrm{m}$ x $100 \mu \mathrm{m}$ square of width $1 \mu \mathrm{m}$ were also patterned to aid in finding the pattern after development. To account for some variation during the patterning, development and etching process, several copies of the same pattern were made on each sample with a variety of doses. This "dose array" improved the effective yield on samples throughout the process.

The pattern was developed using either 25\% TMAH developer for 1 minute or CD-26A developer for 3 minutes followed by a rinse in a 1:9 mixture of CD-26A:DI water for 1 minute and then a final rinse in deionized water. After development, the alignment marks were clearly visible using a standard optical microscope and arrays with smaller pitches had sufficient feature density to be visible in some cases. This optical inspection served as a good indicator of patterning and development success. Further inspection via scanning electron microscopy was performed in some cases to verify that the nanoscale features developed properly. 


\section{Reactive Ion Etching}

A Unaxis 790 reactive ion etching system was used to transfer the pattern through the $\mathrm{Si}$ and $\mathrm{CFO}$ films through a two-step etching process. An initial etch using a mixture of $\mathrm{O}_{2}$ and $\mathrm{SF}_{6}$ was performed to selectively remove the exposed amorphous Si capping layer. The mixture was calibrated sample-tosample to remove a sufficient amount of Si to prepare the substrate for the next etch. The base pressure of the system was less than $5 \times 10^{-4}$ Torr and the operating pressure was 10 mTorr. The RF power was set to a value to produce a DC bias of $330 \mathrm{~V}$, with powers ranging from 100 to $125 \mathrm{~W}$. The Si cap is partially removed during the process and the patterned HSQ pillars are preserved. A second etch is performed using the same RIE system, with $50 \mathrm{sccm}$ Ar flow, 8 mTorr operating pressure, $500 \mathrm{~W}$ RF and a $750 \mathrm{~V}$ DC bias. This produces a CFO etch rate of 3-5 nm/min with some variation from sample to sample. Si and HSQ rates are considerably higher. The etching time was varied so as to fully remove the CFO layer and re-expose the substrate for each sample. Typical etch times were 150-210 seconds in total. The completion of the etch was confirmed through AFM inspection of the arrays, which showed that stepedges from the original substrate treatment were visible and that the surface topography no longer showed the island morphology that develops during the growth of the CFO film on the Nb:STO substrate. 


\section{Thin Film Characterization Techniques}

\section{X-Ray Diffraction}

\section{Diffraction System}

X-ray diffraction was employed to characterize thin film thickness, strain, structural phase, and other properties using a Rigaku Smartlab diffractometer. The system is equipped with a $\mathrm{Cu}$ x-ray source, parallel beam optics and optional monochromators, which produces a monochromatic x-ray beam with wavelength of $1.54 \AA{ }^{128}$ Samples are mounted on a goniometer with 4-circle diffraction capabilities. These capabilities allow for determination of both out-of-plane and in-plane lattice parameters of the thin film samples prepared using the PED system.

For all results presented in this work, the Ge (220) two-bounce monochromator was used during measurement. Incident and receiving optical slits were generally between $0.2 \mathrm{~mm}$ and $2 \mathrm{~mm}$ for diffraction measurements, depending on the signal intensity and angular resolution desired in the measurement. For most measurements, all slits were set to $1 \mathrm{~mm}$. Scan speeds were set such that integration times would be sufficient to reduce the effects of background noise in the data. In most cases, one second integration time (i.e. time to collect a single data point) per step was sufficient, though some $\mathrm{CoFe}_{2} \mathrm{O}_{4}-\mathrm{BiFeO}_{3}$ nanocomposite films required longer integration time due to the low density of $\mathrm{CoFe}_{2} \mathrm{O}_{4}$ pillars. Prior to measurement, the samples were mounted on the goniometer on an amorphous glass slide. An standard sample alignment procedure was executed to align the optics with the sample weight and tilt. Further alignment was performed manually to align the goniometer to the single crystal substrate diffraction peak by iteratively scanning the goniometer $\omega$ and $2 \theta$ angles with decreasing slit sizes. This procedure removed the impact of substrate miscut or misorientation on the final measurement, i.e. peak intensities and shapes. 


\section{Diffraction Measurement Techniques}

\section{1. $2 \theta$ scan}

For many films, out-of-plane diffraction measurements were performed by scanning $\omega$ and $2 \theta$ such that the step in $\omega, \Delta \omega$, was equal to half the step in $2 \theta, 1 / 2 \Delta(2 \theta)$. This configuration is analogous to the traditional $\theta / 2 \theta$ scans in powder diffraction but allows for the alignment to the substrate peak that is needed for single crystal substrates. In epitaxially grown films, it is common to see what are referred to as thickness fringes. These thickness fringes are oscillations in the diffracted intensity around a thin film diffraction peak in reciprocal space which result from refraction of the incoming $x$-rays at the interface between the film and the substrate. The periodicity of the thickness fringes, $\Delta \eta_{i}$, in radians is given by:

$$
\Delta \eta_{i}=\frac{\lambda}{2 T \cos \theta_{B}}
$$

where $T$ is the film thickness, $\theta_{B}$ is the Bragg diffraction angle and $\lambda$ is the x-ray wavelength. ${ }^{129}$ Empirically, these fringes are measured most commonly in epitaxial films that grow with few defects and a coherent or semi-coherent interface with the substrate. The presence of these fringes is indicative of reasonably high quality of thin films. In some cases, the thicknesses of the films were calculated using the periodicity of the thickness fringes.

\section{Reciprocal space mapping}

Reciprocal space maps are commonly used to characterize the in-plane strain conditions of an epitaxial film. In these measurements, diffracted intensity is measured over a two-dimensional parameter space. Most commonly, the goniometer coordinates that are varied are $2 \theta$ and $\omega$. This approach allows for the determination of both in-plane and out-of-plane reciprocal lattice components using the formulas ${ }^{129}$ :

$$
\begin{aligned}
& Q_{x}=2 \pi / \lambda(\cos (2 \theta-\omega)-\cos \omega) \\
& Q_{z}=2 \pi / \lambda(\sin \omega+\sin (2 \theta-\omega))
\end{aligned}
$$


Many samples in this work were characterized via reciprocal space mapping in order to determine the degree of strain relaxation in the thin films which were grown.

To perform reciprocal space maps, an asymmetric diffraction condition was generally chosen to reduce the degrees of freedom in the system. A particular substrate diffraction peak containing a nonzero in-plane coordinate was chosen which would also be in the vicinity of a thin film peak. For films grown on perovskite substrates, such as $\mathrm{SrTiO}_{3}$ and $\mathrm{LaAlO}_{3}$, the $\{103\}$ planes were usually ideal. Using the Diffraction Space Simulation (DSS) package in the Rigaku Guidance software system, the $2 \theta$ diffraction peak for the substrate was calculated, along with the angle $\chi$, which becomes non-zero if the reciprocal lattice point has a non-zero in-plane component. If a symmetric scan were performed, the calculated value for $\chi$ from the DSS package would be entered into the goniometer control. However, in an asymmetric scan, the value for $\omega$ is not equal to $2 \theta / 2$, but instead given by:

$\boldsymbol{\omega}=\frac{2 \boldsymbol{\theta}}{2}-\chi$

which also satisfies the diffraction condition. ${ }^{129}$ The values for $\omega$ and $2 \theta$ were then entered into the goniometer control and a $360^{\circ}$ scan of the goniometer variable $\varphi$ is performed to find the diffraction peaks that satisfy this Bragg condition. In general, there are multiple peaks present in the $\varphi$-scan since the Bragg condition for $\{\mathrm{H} 0 \mathrm{~L}\}$ is satisfied by $(\mathrm{H} 0 \mathrm{~L}),(0 \mathrm{H} \mathrm{L}),(-\mathrm{H} 0 \mathrm{~L})$ and $(0-\mathrm{H} \mathrm{L})$ and a scan in $\varphi$ will pass through all four such diffraction conditions. One of the peaks was chosen for $\varphi$ and the built-in Guidance alignment about the substrate peak was performed to optimize the value of $\omega$ accounting for substrate miscut. Finally, a reciprocal space map is performed by independently scanning $\omega$ and $2 \theta$, with optical slit sizes similar to those used for the scans described above. The range of $2 \theta$ and $\omega$ values is chosen to incorporate all the desired substrate and film peaks, with integration times sufficient to reduce the effects of statistical noise. The measurement is made by starting at a particular $(2 \theta, \omega)$ coordinate given by:

$(2 \theta, \omega)=\left(2 \theta_{B}, \omega_{B}-\delta\right)$ 
where $2 \theta_{B}$ and $\omega_{B}$ are the coordinates of the Bragg peak chosen and $\delta$ is an offset angle. At each starting point, a $2 \theta / \omega$ scan is performed over the chosen range of $2 \theta$ with the step in $2 \theta$ equal to twice the step in $\omega$. Data analysis is performed using the Rigaku 3D Explore software, which uses the formulas given in Equation 4-4 to convert the goniometer coordinates, $(2 \theta, \omega)$ to reciprocal space coordinates, $\left(Q_{x}, Q_{z}\right)$. A schematic showing the configuration of these coordinates is shown in

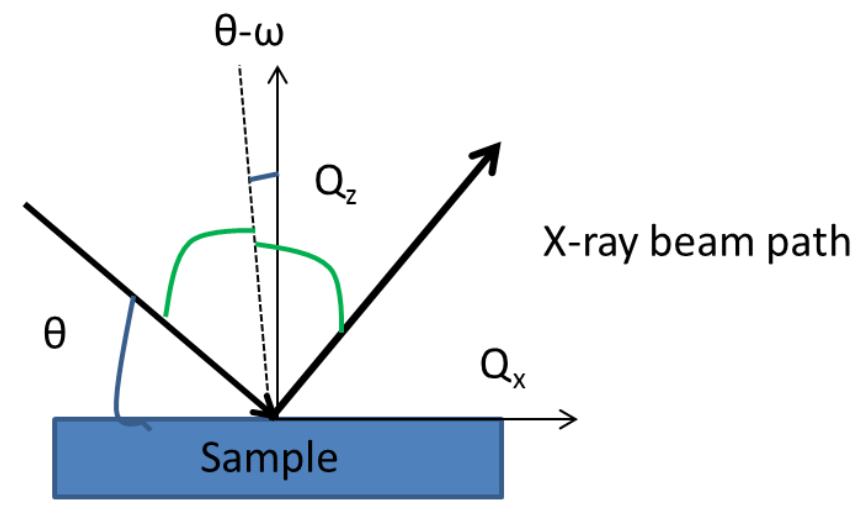

Figure 4-1. Geometry of diffraction system for reciprocal space maps. Green angle marks indicate equivalent angles centered about the dashed line.

\section{X-ray reflectivity (XRR)}

X-ray reflectivity (XRR) is a common technique used to determine the thickness of films. ${ }^{129}$ Just as thickness fringes occur around the (H K L) diffraction peaks when there is a smooth interface with a substrate, oscillations also occur around the (000) point in reciprocal space. The (000) point occurs at $\omega=2 \theta=0^{\circ}$. Scattering of incoming x-rays near the reciprocal space origin is governed primarily by refraction, rather than by Bragg diffraction. By performing a $2 \theta / \omega$ scan from $0^{\circ}$ to approximately $5^{\circ}$, it is possible to measure the thickness, or Kiessig fringes, which are produced due to refraction between the substrate and film interface. ${ }^{129}$ At extremely small angles - generally less than $1^{\circ}$ - the incoming beam is totally reflected by the air-film interface. The angle at which total external reflection ceases is called the critical angle, $\alpha_{c}$, and is given by:

$\sin \alpha_{c}=\sqrt{2(1-n)}$ 
where $n$ is the refractive index of the film. The refractive index is a density dependent quantity, so different materials have different critical angles, but all are generally between $0.3^{\circ}$ and $1.0^{\circ}$. At angles less than $\alpha_{c}$ the incoming beam is fully reflected and measured by the detector. For angles greater than $\alpha_{c}$, the intensity decays with increasing angle and undergoes oscillations which are governed by the thickness of the film. The thickness of the film, $T$, is given by:

$\boldsymbol{T}=\frac{2 \pi}{\Delta \boldsymbol{Q}_{z}}$

where $\Delta Q_{z}$ is the period of oscillation in reciprocal space. ${ }^{129}$ Film roughness and the difference in density at the interfaces affects the rate of decay of the reflected intensity, with rougher films decaying more rapidly with increasing angle. For multilayer films, a more complex reflectivity profile results due to the additional interfaces present in the sample. Theoretical models are generally very effective for fitting the profiles and determining the thicknesses and densities of each layer, along with the interfacial roughness between layers.

X-ray reflectivity measurements are fully automated in the Rigaku Guidance software package. The sample was mounted on the stage and the optical alignment routine was performed with the Ge (220) 2bounce monochromator mounted. The default XRR routine in the system scans over a range of $\left[0^{\circ}, 10^{\circ}\right]$ in $2 \theta$, with slit sizes $0.05 \mathrm{~mm}$ for both the incident and first receiving slits. In practice, the scattered beam was generally below the noise level at angles greater than about $5^{\circ}$ and the scans were often aborted above those angles to save time. The scan time (minutes for a $10^{\circ} \mathrm{scan}$ ) was chosen depending on the material of interest, the expected roughness and the calibrated thickness of the film. For most single layer samples with thicknesses of $50 \mathrm{~nm}$ or less, a scan time of 20 minutes was sufficient. Curve fits of the intensity profile were then performed using the Rigaku GXRR analysis software, which allows for calculation of film thickness, density and surface roughness. A screen capture of a fit performed using the software package is shown in Figure 4-2. Although CFO-BFO nanocomposites are not uniform, if the area fraction of CFO is sufficiently small a reflectivity oscillation from the BFO matrix is generally found 
to be present. In these samples, fitting the profile by assuming that the material was composed entirely of BFO produced reasonable matches to the measured profiles and was taken to be an accurate model.

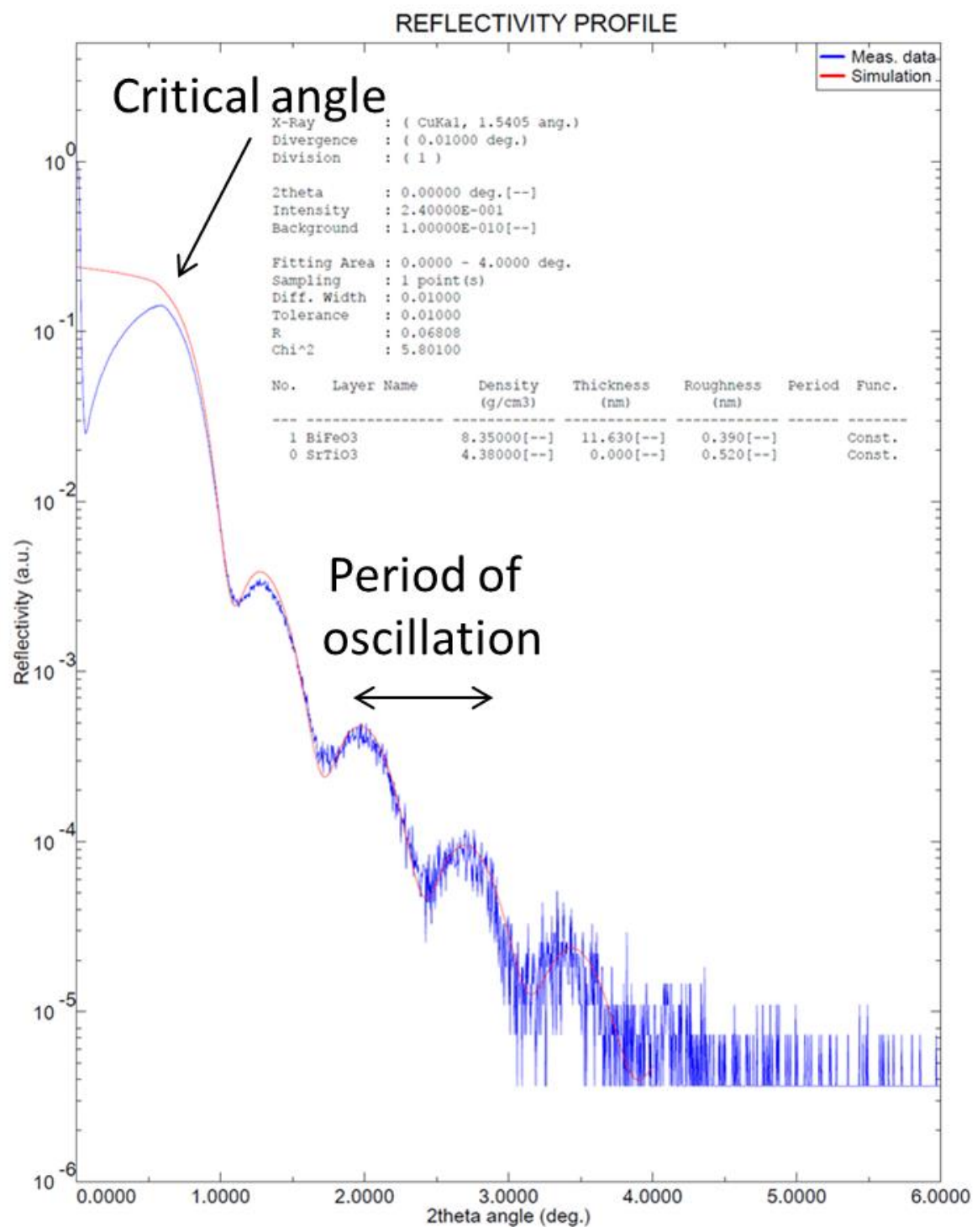

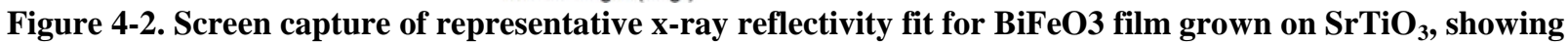
the critical angle and oscillation period.

\section{Magnetic Characterization}

The magnetic properties of the samples were characterized via vibrating sample magnetometry (VSM) and magnetic force microscopy (MFM). MFM will be discussed in detail below, as it is a scanning probe microscopy technique. The VSM is a commonly used system, first developed by Simon 
Foner in 1959 , to measure the total magnetization of a material in response to an applied magnetic field. ${ }^{130}$ VSM systems operate by mounting the sample along a nonmagnetic rod, which is placed between the poles of an electromagnet. The rod is driven at a specific vibrational frequency that moves the sample along the axis of the rod. A pickup coil is mounted such that the sample oscillates within the coil, which induces an alternating electromotive force (emf) in the coil. The frequency of the emf is equal to the vibrational frequency of the rod and the amplitude is proportional to the magnetization of the sample. The measured emf is compared to a reference signal with the same frequency as the vibrating sample using a lock-in amplifier. ${ }^{131}$ In this fashion, the instantaneous magnetization of the sample is detected. Magnetic hysteresis loops are performed by applying an external field with the electromagnet in the system, scanning the field in time and measuring the magnetization of the sample at regular intervals.

VSM measurements in this work were performed using two cryogenic Quantum Design systems, the Physical Property Measurement System (PPMS) and the VersaLab. The PPMS is a cryogenic system capable of applying magnetic fields up to 7 Tesla using a superconducting coil inside of a liquid helium dewar. The system is capable of variable environmental temperatures from less than $5 \mathrm{~K}$ to $400 \mathrm{~K}$, which allows for temperature dependent magnetization measurements and characterization of samples with magnetic phenomena that occur below room temperature. The VSM is sensitive to sample magnetizations as small as $10^{-7} \mathrm{emu}$, which is sufficient to detect the magnetization of ferromagnetic films on $5 \times 5 \mathrm{~mm}$ substrates only a few nanometers thick.

The VersaLab system operates in a similar fashion to the PPMS, but does not use liquid helium or a superconducting coil. Instead, a cryogenic pump is used to achieve minimum temperatures of $50 \mathrm{~K}$. The applied field is limited to 3 Tesla and the system is sensitive to magnetizations as small as $10^{-6}$ emu. For many applications, however, these capabilities are more than sufficient. For room temperature measurements that did not require applied fields of greater than 3 Tesla, the VersaLab so as not to unnecessarily consume the liquid helium that is needed to operate the PPMS. 
Hysteresis loops were performed by mounting samples on two different sample holders. Samples were mounted on an acrylic plastic holder with double sided tape for in-plane VSM measurements. For perpendicular measurements, samples were mounted in a metallic diamagnetic holder with a notch cut into it to hold a $0.5 \mathrm{~mm}$ thick substrate. The substrate was held in place by an acrylic insert that provided a pressure fit to maintain stability. For in-plane measurements, a $5 \mathrm{~mm}$ bore coil was used that provided ideal signal to noise characteristics. For out-of-plane measurements, a larger bore coil was used to allow the entire $5 \mathrm{~mm}$ square substrate to be measured without the need for dicing. Examples of all these configurations are shown in Figure 4-3.

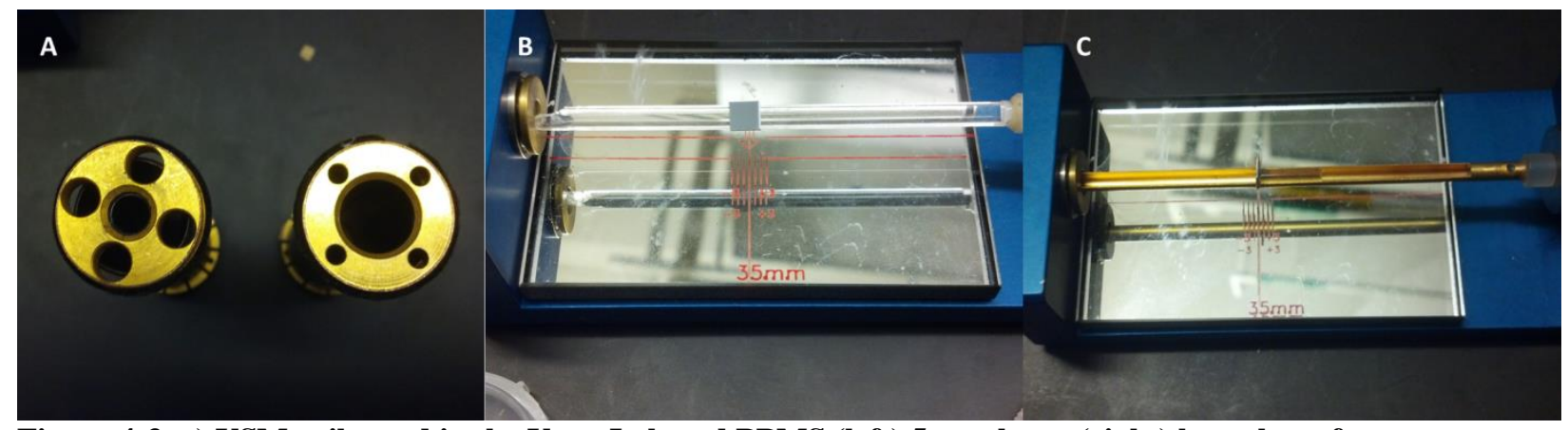

Figure 4-3. a) VSM coils used in the VersaLab and PPMS (left) $5 \mathrm{~mm}$ bore, (right) large bore for perpendicular measurements; (b) Example of sample mounted for in-plane VSM measurement; (c) Example of sample mounted for perpendicular VSM measurement.

\section{Electron Microscopy}

\section{Scanning Electron Microscopy}

Scanning electron microscopy (SEM) was employed throughout this work for nanoscale imaging of both EBL patterned samples and unpatterned nanocomposites. Several different field-emission SEM systems were employed depending on the site of the research and the availability of the systems. A Zeiss Supra system at the University of Virginia, along with a Zeiss Ultra-60 and a Zeiss NVision 40 dual-beam SEM/focused ion beam (FIB) system at the NIST Center for Nanoscale Science and Technology (CNST) were all used for SEM imaging. Imaging was performed using the in-lens detector to measure secondary electrons emitted from the sample. ${ }^{33}$ Due to the insulating nature of nanocomposite films, low electron 
gun voltages of $2 \mathrm{kV}$ were used whenever possible. SEM imaging was employed exclusively for observation, with no efforts made to perform quantitative analysis of the materials.

\section{Transmission Electron Microscopy}

Transmission electron microscopy (TEM) is a valuable technique for the characterization of thin films, providing atomic resolution of crystals under proper conditions. In this technique, a high energy electron beam (100-300 keV) bombards a sample that has thickness of less than $100 \mathrm{~nm}$. By focusing the beam that is transmitted through the sample, it is possible to acquire structural and chemical information of the portion of the sample that the electrons passed through. It is also possible to perform chemical analysis of the samples by analyzing the energy spectra of x-rays that are emitted when the electron beam excites the atoms in the crystal. In this section, I will discuss the experimental techniques employed in this work and describe the analytical procedures used to interpret the results. Dr. Kerry Siebein, a microscopy expert at the National Institute of Standards and Technology helped prepare the samples and acquire the TEM images and data presented here.

\section{Sample Preparation}

To prepare the samples for TEM analysis, a dual-beam scanning electron microscope (SEM)/focused ion beam (FIB) system was used to extract a cross-sectioned lamella. An FEI Helios 650 system was used for this work with a Ga ion source and a field emission electron gun. The system is equipped with several gas injection system (GIS) sources, which are used to inject a variety of different precursor metal-organic gases for deposition. To deposit these materials, either the SEM or FIB gun is used to crack the precursor gas on the surface of the sample. The system is also equipped with an Oxford Omniprobe sample manipulator, which is used during the TEM sample preparation process to lift lamella from the substrate.

Figure 4-4 shows the progression of the lamella extraction process from a patterned nanocomposite. For the patterned nanocomposite, initial $1 \mu \mathrm{m}$ tall, $1 \mu \mathrm{m}$ diameter Pt metallic pillars were deposited around the array of interest for use as alignment marks using the electron gun with a beam current of 800 
$\mathrm{pA}$ and $5 \mathrm{kV}$ accelerating voltage. Both patterned and unpatterned nanocomposites were then coated with approximately $100 \mathrm{~nm}$ of amorphous carbon using a Gatan Precision Etching and Coating System. The carbon serves as a conductive coating to reduce the effects of charging that occur due to the insulating nanocomposite film. The samples were then placed in the dual-beam system for the lift-out process. A 2 $\mu \mathrm{m}$ thick initial Pt rectangle with length of $20 \mu \mathrm{m}$ and width of $2 \mu \mathrm{m}$ was deposited along the <110> surface axis using the ion beam source with $30 \mathrm{kV}$ and $0.23 \mathrm{nA}$ beam conditions. This serves as a protective coating during the extraction process. An image of the rectangle on the patterned sample is shown in Figure 4-4(A). The FIB gun is then used to etch a trench into the substrate surrounding the Pt rectangle, with gun conditions set to $30 \mathrm{kV}, 9.3 \mathrm{nA}$. A reduced beam current of $2.5 \mathrm{nA}$ is used to clean any residual material from the trench. The sample is then tilted to undercut the substrate beneath the Pt rectangle. Using the Omniprobe manipulator, a micron scale tip is then mounted to the lamella by depositing Pt using the ion beam source with a beam current of $24 \mathrm{pA}$ at the interface between the probe and lamella. This step is shown in Figure 4-4(B). With the probe attached, the lamella is then cut from the substrate using the FIB with a beam current of $2.5 \mathrm{nA}$. The sample is then attached to a Cu TEM grid by depositing Pt with the ion beam source with $80 \mathrm{pA}$ current. The probe is then cut free using the FIB, leaving the lamella attached to the grid. This is shown in Figure 4-4(C). Finally, a portion of the sample is progressively thinned from its initial $2 \mu \mathrm{m}$ thickness to less than $100 \mathrm{~nm}$ using the ion gun with $230 \mathrm{pA}$ beam current. A view of the final thinned lamella is shown in Figure 4-4(D). 


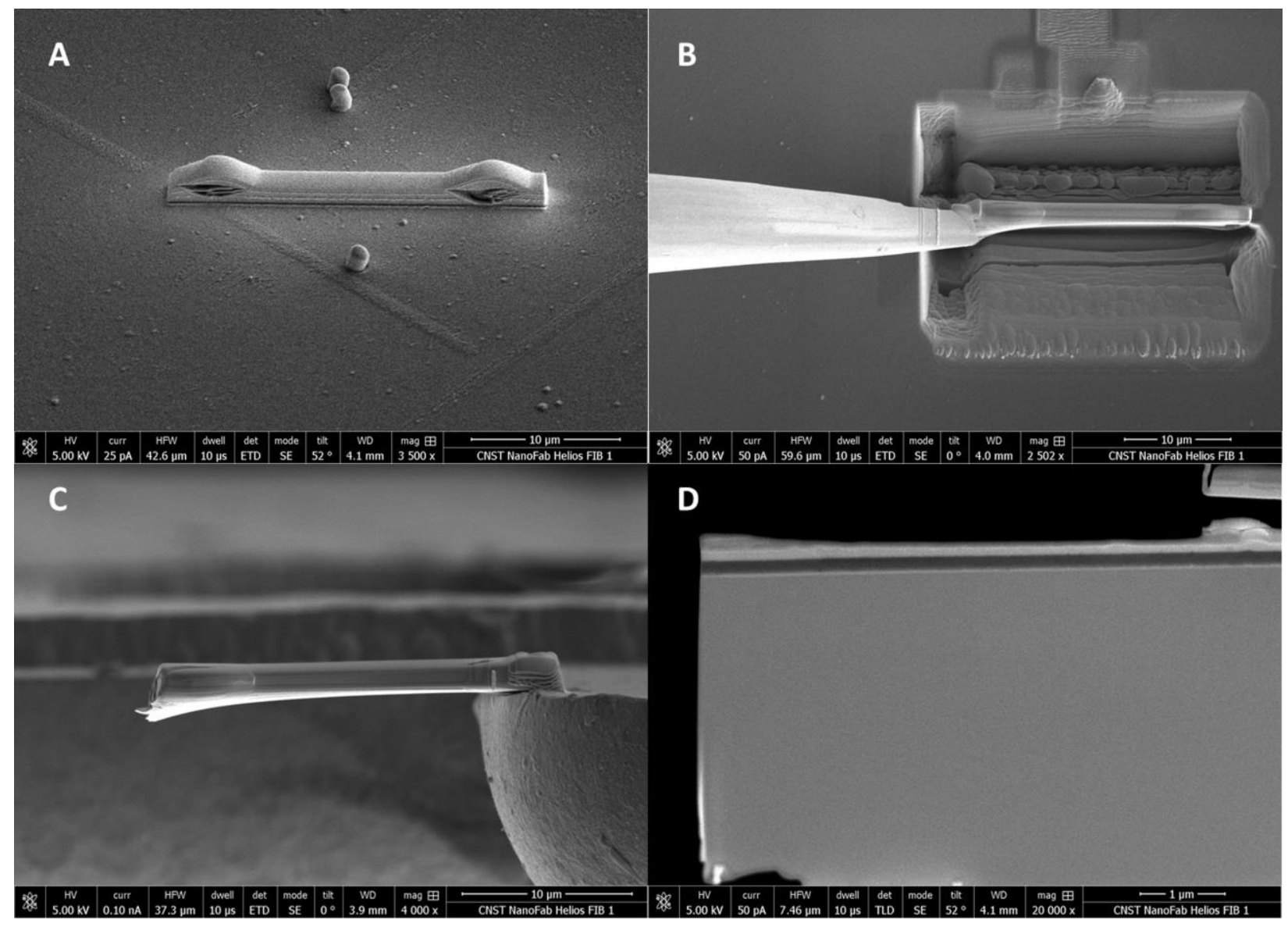

Figure 4-4. Preparation process for TEM lamella. A) Deposition of Pt protective barrier along $\langle 110\rangle$ axis. B) Trench milling and Omniprobe attachment to lamella. C) Mounting of lamella on Cu grid. D) View of final thinned lamella.

\section{High resolution TEM Analysis}

Cross-sectional high-resolution TEM (HRTEM) was used in this work to characterize the interfaces of both patterned and unpatterned $\mathrm{CoFe}_{2} \mathrm{O}_{4}(\mathrm{CFO})-\mathrm{BiFeO}_{3}(\mathrm{BFO})$ nanocomposites. During HRTEM imaging, the parallel electron beam illuminates the entire region of interest, with the sample magnification determining the area that is illuminated. In the image plane on the detector, the phase shift of electron waves coherently diffracted through by the specimen is used to reconstruct the real-space crystal structure of the sample. ${ }^{132}$ This reconstruction occurs as a result of the superposition of the diffracted electron waves on the surface, which interfere either constructively, giving a bright region, or destructively, giving a dark region. To perform these measurements, the sample is tilted until the incident beam is parallel to 
the desired crystal zone axis and the electromagnetic lenses are then focused and the beam stigmation adjusted to achieve optimal resolution. In the case of a TEM system that does possess aberration correction for the magnetic lenses (as is the case in this work), a slight defocus of the transmitted beam must be used to achieve optimal resolution in the image plane. The thickness of the sample affects the intensity of the transmitted beam, with thicker regions providing lower contrast than ideal thin regions. This is a result of absorption of the electrons through incoherent processes as they travel through the width of the sample.

Using HRTEM, cross-sectional images of both patterned and unpatterned samples were examined along the [110] Nb-doped $\mathrm{SrTiO}_{3}(\mathrm{Nb}: \mathrm{STO})$ substrate zone axis. This axis was chosen due to the nature of the faceting of the CFO pillars, which produce $\{110\}$-type in-plane facets. Thus, by examining the samples along this zone axis, the CFO pillars will have uniform thickness along the beam axis. The interfaces between the CFO pillars and the Nb:STO substrate, the BFO matrix and the substrate, and the CFO pillar and the BFO matrix were all characterized to determine the coherency of the interfaces. Bright-field images with magnification of between 56,000x and 620,000x were acquired for both samples. Image processing was performed using the Gatan Digital Micrograph analysis software.

To analyze crystal faces and measure lattice strain, fast Fourier transform (FFT) analysis of the images was performed. These images are analogous to the selected-area electron diffraction images that are often used to measure lattice parameters using TEM. By measuring the distance in reciprocal lattice units between indexed peaks, it is possible to calculate the lattice parameter of the pillar and the pseudocubic lattice parameter of the matrix. Line profiles of the reciprocal space peaks were calculated and non-linear least-squares fits were performed to determine the peak position. The uncertainty in the calculated lattice parameters was determined based on the uncertainty in the fit of the peak position. 


\section{High-angle Annular Dark Field STEM}

High-angle annular dark field scanning TEM (HAADF-STEM) is a commonly used technique to obtain chemically sensitive contrast from a sample. In HAADF-STEM, unlike TEM, the electron beam is focused to the smallest achievable area and scanned across the region of interest to achieve a spatial reconstruction of the sample. ${ }^{132}$ The image is reconstructed using incoherent elastically-scattered electrons. These electrons scatter to higher angles than those in phase-contrast TEM. By placing an annular detector in the beam path that allows the unscattered beam to pass through, the incoherently scattered electrons are measured. The intensity of the scattering at each scanning point is used to generate the HAADF-STEM image. This approach is referred to as "Z-contrast imaging", because the scattering intensity is proportional the atomic number, $Z$, of the atom that scatters the electron. Thus, HAADFSTEM images are more readily interpreted to understand chemical composition of a material than are HRTEM images.

The same samples that were characterized using HRTEM were also analyzed using the HAADFSTEM detector in the Titan microscope. The beam was focused to a minimum probe size of approximately $2 \AA$ and images with 2000x2000 pixels were acquired at various magnifications. Due to the thickness of the samples, it was not possible to obtain atomic resolution using STEM due to electron absorption through the film. The Gatan TEM Imaging and Analysis software on the Titan microscope was used to acquire the images. To analyze the images, the Fiji image processing software, which is an opensource platform based on ImageJ was employed. ${ }^{133}$

\section{Energy Dispersive X-ray Spectroscopy}

During TEM analysis of a sample, the electron beam incident on the sample in some cases may excite core level electrons of the atoms in the sample, ionizing the atoms. This excited state will then rapidly relax back to a ground state electronic configuration, which results in the emission of an x-ray. Through the use of energy dispersive x-ray spectroscopy (EDS), it is possible to use measure the energy of the emitted x-rays to determine the chemical composition of the sample. By placing an x-ray spectrometer 
with line of sight view to the sample, an x-ray spectrum can be acquired from the sample using the electron beam as the excitation probe.

Using STEM mode in this work, EDS maps were taken of both patterned and unpatterned nanocomposites with spatial resolution of 1-2 $\mathrm{nm}$. The electron beam was scanned over the sample and an EDS spectrum was acquired at each point in a grid, with side lengths of 20 to $50 \mathrm{~nm}$ depending on the feature. An example of one such EDS spectrum is shown in Figure 4-5. A HAADF-STEM image was initially used to select the region of interest, and the HAADF intensity at each point was also measured during the map to correlate structural features with the chemical composition. Measurements using the Gatan TEM Imaging and Analysis (TIA) software package took between 30 and 60 minutes and were automatically corrected for drift by imaging a reference region outside of the region of interest. After data collection, post-processing was performed using TIA. The intensity of the various x-ray peaks from different elements were generated by integrating the area of the peak over the width of the peak. The integration regions are shown by the green rectangles in Figure 4-5. A spatial map of these intensities was automatically generated by the software as a grayscale image. This image was then edited using the GNU Image Processing (GIMP) software to produce a false color image which overlaid multiple elemental maps into a single image with different colors. 


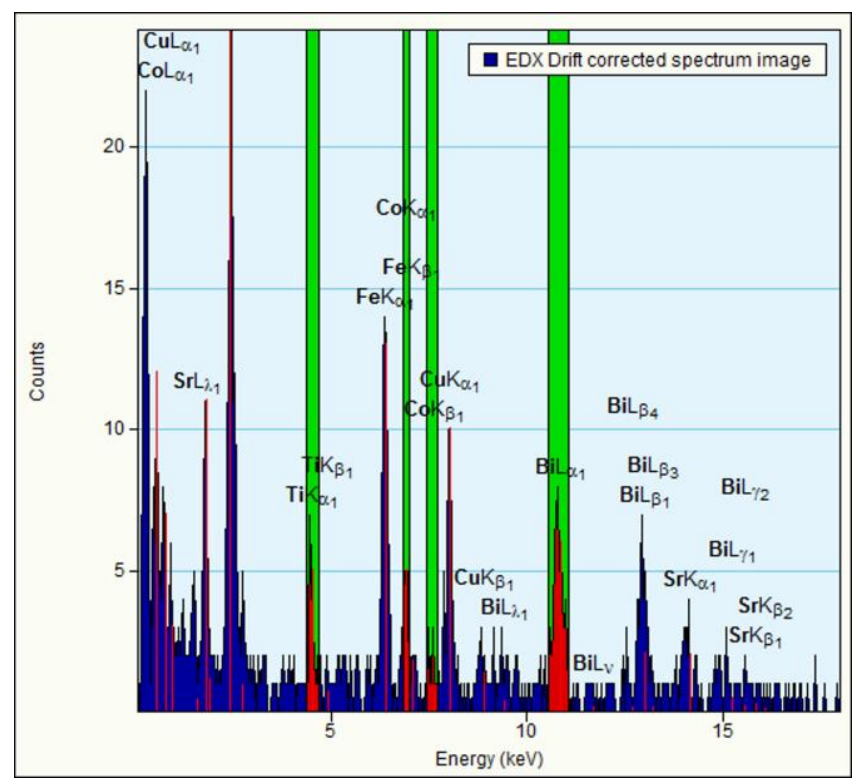

Figure 4-5. Energy Dispersive X-Ray Spectroscopy (EDS) spectrum showing relevant x-ray peaks for $\mathrm{CoFe}_{2} \mathrm{O}_{4}-\mathrm{BiFeO}_{3}$ nanocomposite grown on $\mathrm{SrTiO}_{3}$.

\section{Description of Microscope}

All TEM and STEM experiments were performed on an FEI Titan 80-300 scanning transmission electron microscope. The microscope uses a high-brightness Schottky field emission source, with accelerating voltages between $80 \mathrm{kV}$ and $300 \mathrm{kV}$. All images and spectra in this work were acquired at $300 \mathrm{kV}$ accelerating voltage. The system does not have aberration correction, limiting resolution to between 1 and $2 \AA$ in STEM mode. A Fischione HAADF-STEM detector is used for STEM image acquisition. For EDS measurements, the system is equipped with an EDAX $\mathrm{Si}(\mathrm{Li})$ detector that can be inserted above the sample plane. All images are acquired using a Gatan Orious camera with 2000-by2000 pixel resolution.

\section{Scanning Probe Microscopy}

Scanning probe microscopy (SPM) is a common technique to characterize the morphology of thin film samples, along with their magnetic and ferroelectric properties. Initially developed by the inventors of the scanning tunneling microscope (STM) ${ }^{134}$, atomic force microscopy (AFM) is a simpler technique which involves the scanning of a nanoscale tip mounted on a cantilever along the surface of a sample 
while either maintaining constant contact with the surface (contact mode) or oscillating the tip at the mechanical resonance frequency of the cantilever (tapping mode). ${ }^{135,136}$ For most topography applications, the tapping mode technique allows for improved resolution and reduced wear of the tip. ${ }^{136} \mathrm{~A}$ laser beam is reflected off the cantilever to measure small displacements due to changes in the tip-surface interactions. Creative uses of AFM systems have been developed to measure other nanoscale forces. These include magnetic force microscopy $(\mathrm{MFM})^{137}$, which uses a magnetic material on the probe tip to detect small variations in the magnetic field emanating from the sample, and piezoresponse force microscopy (PFM) ${ }^{138,139}$, which uses the tip as an electrical contact to the surface to probe the ferroelectric properties of a thin film.

\section{Description of Systems}

SPM was employed to characterize the thin film properties throughout this work. Several different scanning probe systems were used over the course of the work, but final analysis for this work was performed exclusively on either an NT-MDT system or Asylum Research Cypher system. The NT-MDT system was used for initial magnetic force microscopy measurements prior to the purchase of the Cypher

system and has been described elsewhere. ${ }^{140}$ The Asylum Research Cypher scanning probe microscope is designed to permit easy operation of a variety of scanning probe techniques. A photograph of the entire system is shown in Figure 4-6. A video camera system with high resolution zoom is employed to align the laser detection system and to find features on the surface of the sample. The standard open air cantilever holder is designed to allow for up to $\pm 10 \mathrm{~V}$ to be applied to the cantilever. For higher applied voltages that are necessary for some PFM applications, a separate cantilever with high voltage grounding is provided, though that cantilever was not needed in this work. Samples are set on a small metallic disk for general measurements or mounted using colloidal silver paint for PFM measurements, which is held in place using a magnetic chuck. The entire scanning probe system is housed inside of an environmentally controlled cabinet that maintains a temperature 5-10 degrees Celsius above ambient temperature, which minimizes thermal drift. 


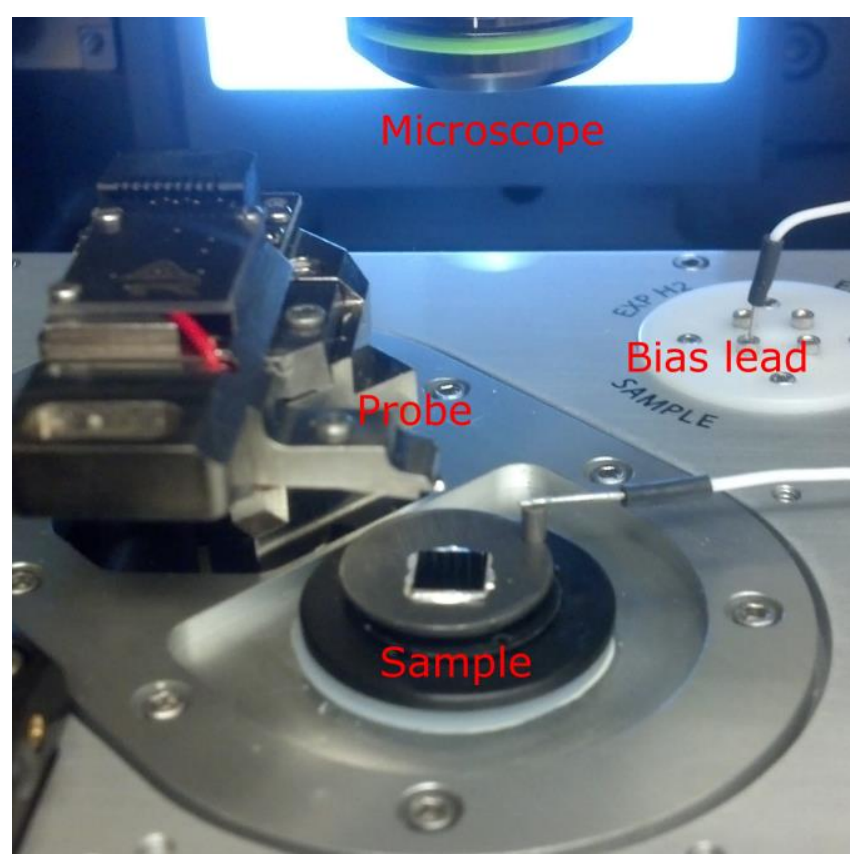

Figure 4-6. Photograph of Asylum Cypher configuration for piezoresponse force microscopy (PFM) measurement. For non-PFM measurements, the bias lead was not used.

For conventional AFM measurements, an AC160TS tip was employed. These tips have radius of curvature of approximately $9 \mathrm{~nm}$. The free air resonance frequency of these tips was approximately 300 KHz. For topography measurements, the AC semi-contact mode was used. The set point voltage was less than $0.8 \mathrm{~V}$, which controlled the force applied between the tip and surface. Scanning rates were chosen to keep tip scanning rates in the range of $1-3 \mu \mathrm{m} / \mathrm{sec}$.

\section{Magnetic Force Microscopy}

Magnetic force microscopy (MFM) is a scanning probe technique used to measure the magnetic domain structure of thin films and nanostructures. First developed in $1987,{ }^{137}$ the technique uses an AFM tip which has been coated in a ferromagnetic material, such as $\mathrm{CoCr}$ alloy, to measure the magnetic field near the surface of the sample. To perform these measurements, an initial scan is made with the tip in semi-contact (AC) mode to measure the topography of the sample surface. After this measurement, the tip is then lifted off the surface by a constant distance and a second $\mathrm{AC}$ measurement is made. By measuring 
the phase shift in the response of the cantilever during the second pass, it is possible to measure the magnetic field at that height above the surface of the sample. If the tip is magnetized such that its magnetization is aligned perpendicular to the surface of the sample, it will be sensitive only to the magnetic field along that axis. ${ }^{141}$ In this way, MFM is used primarily to measure the out-of-plane magnetization of a film. However, with sufficient resolution, it is possible to measure the in-plane magnetization by observing the curling of the magnetic field around a feature. Interpretation of these images generally demands some a priori knowledge of the expected magnetic properties of the material.

For MFM measurements in this work, the Cypher system was used exclusively for all nanocomposite samples, while the NT-MDT system was used for measurements on uniform $\mathrm{CoFe}_{2} \mathrm{O}_{4}$ films. This section will describe the procedures for the Cypher system. Settings for the NT-MDT system were analogous. Using the Cypher system, an ASYMFM tip was installed, which is coated in CoCr alloy and has a radius of curvature of approximately $50 \mathrm{~nm}$. The cantilever free air resonance frequency is approximately 70 KHz. Scans were performed on nanocomposite samples with lift heights of 20-30 nanometers so as to prevent the rough surface from interfering with the phase response during measurement. In the Cypher system, the "napping" mode was employed to perform the two-pass routine. The initial topography scan was performed in the same manner as in conventional AFM, followed by the second pass. In the second pass, the driving amplitude was reduced by a factor of 2 and the "nap height" was set to the desired distance. All other control parameters were held constant. Scans were performed over a variety of scan sizes, with the scanning speed and line count chosen to produce small enough step sizes to resolve all features and minimize tip damage. In cases where the sample was demagnetized prior to measurement, the Versalab system was used to first saturate the field in the out-of-plane direction and then perform a de-Gaussing routine by applying a field that alternated direction and gradually decayed in amplitude until it approached zero field. 


\section{Piezoresponse Force Microscopy}

Piezoresponse force microscopy (PFM) is a common technique for nanoscale characterization of

ferroelectric and piezoelectric thin films, first demonstrated by multiple groups in the mid-1990s. ${ }^{138,139,142}$ Using an atomic force microscope equipped with a lock-in amplifier, an AFM tip coated with a metallic material is brought into direct contact with a ferroelectric material. A small alternating current (AC) bias voltage is applied to the tip, which induces a deformation in the surface due to the piezoelectric response of the ferroelectric. The displacement of the tip is governed by the equation $d=d_{0}+A \cos (\omega t+\varphi)$, where $d_{0}$ is the equilibrium displacement, $A$ is the amplitude of the mechanical response of the surface, $\omega$ is the frequency of the $\mathrm{AC}$ bias voltage, and $\varphi$ is the phase change that results from the surface polarization. ${ }^{143}$ During the scan, the amplitude, $A$, and the phase shift, $\varphi$, are recorded as data to show the magnitude and direction of the ferroelectric polarization of the sample respectively. The phase, $\varphi$, will be either 0 or $180^{\circ}$ in the case of out-of-plane polarization.

Lateral, or in-plane, PFM is employed to measure the in-plane polarization direction of a ferroelectric material. ${ }^{144,145}$ The Vector PFM technique is a recently developed approach to incorporate lateral PFM into the Asylum Cypher system. ${ }^{146}$ Through repeated measurements, this approach can be employed to measure all three spatial components of the ferroelectric polarization vector, though such an approach was not employed in this work. Instead, a single measurement may be performed to determine both the out-ofplane spatial component and one in-plane component which is parallel to the scan direction. ${ }^{146}$ The Cypher system has a built-in module to perform this measurement. This technique employs a two-pass approach, where an initial PFM measurement is made using the conventional PFM technique outlined in the paragraph above with drive frequency near the out-of-plane contact resonance. To measure the inplane polarization, a second pass is performed at a different drive frequency near the lateral contact resonance. In practice, the out-of-plane resonance is approximately $300 \mathrm{KHz}$, while the in-plane resonance is approximately $1.1 \mathrm{MHz}$. Through this approach, it is possible to visualize one component of the in-plane ferroelectric domain structure of the material, which is sufficient for the work presented here. 
Dual AC resonance tracking (DART) is a new technique developed to improve the signal-to-noise ratio during PFM measurements. ${ }^{147}$ The DART technique was employed for all PFM measurements in this work through the use of an automated software package in the Asylum Cypher system. In this approach, the conductive tip is brought into contact with the surface of the sample and a frequency response curve is taken to measure the cantilever contact resonance frequency. In conventional PFM, the drive frequency would be chosen so as to be slightly less than the resonance frequency, but far enough removed that drift in the resonance frequency will not shift the drive frequency to the opposite side of the Gaussian frequency response peak. In DART measurements, two different drive frequencies are employed on each side of the contact resonance. By using a lock-in amplifier to track the resonance, it is possible to take advantage of the higher amplitude response near the resonance frequency.

Switching-spectroscopy PFM (SS-PFM) is another new technique developed within the past decade to measure the ferroelectric response of a material using a conductive PFM tip. ${ }^{148}$ This approach employs the DART technique and measures the amplitude and phase of the ferroelectric response of the material at various applied voltages. In this manner, a local ferroelectric hysteresis loop can be measured at specific points on the surface of the material. A voltage is applied to the tip according to the representative signal shown in Figure 4-7. Measurements of the ferroelectric response are made both with the voltage applied and at remanence immediately after the bias is applied, to measure both the on and off states in the film. The measurements made with the bias turned off are taken to be the accurate representation of the hysteresis loop for the purposes of measuring the switching characteristics. Several loops are taken at each point and the results are averaged together to improve the statistical precision of the measurements. 


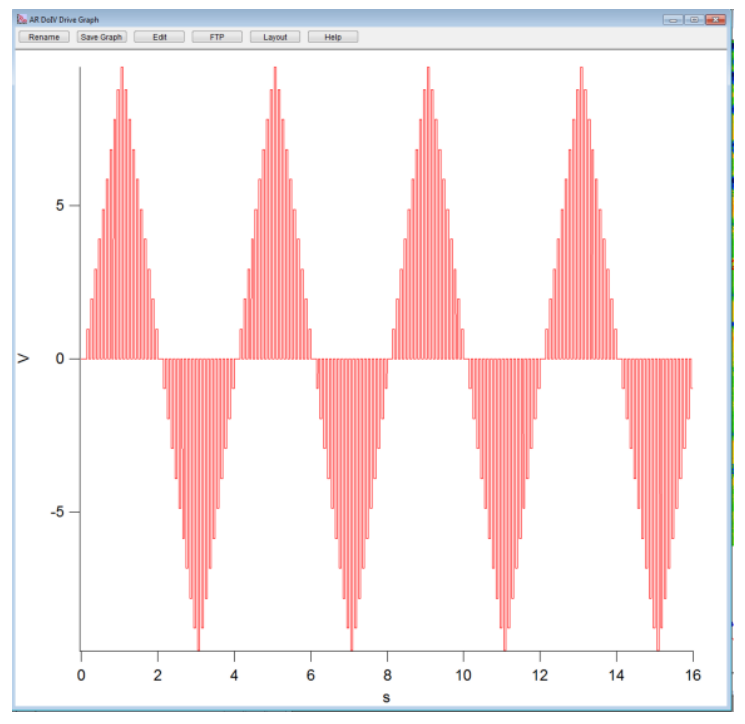

Figure 4-7. Representative applied bias signal for switching spectroscopy PFM measurements.

For all PFM measurements, an ASYELEC-01 tip, which has a Ti/Ir coating, was employed for all types of measurements. The contact resonance between the tip and sample was generally approximately $300 \mathrm{KHz}$ for out-of-plane measurements. For lateral PFM measurements, the contact resonance is typically about 1.1 MHz. Drive voltages were chosen to be as small as possible to produce sufficient signal for measurement, with values typically in the range of $0.5 \mathrm{~V}$ to $2 \mathrm{~V}$ for both out-of-plane and lateral measurements. For DART measurements, scan speed was set to $0.4 \mathrm{~Hz}$ per the manufacturer's recommendation. For conventional PFM measurements, speeds of 0.5 to $0.8 \mathrm{~Hz}$ were generally effective over sufficiently small regions $(<3 \mu \mathrm{m})$. Line densities were chosen to achieve the desired resolution and to allow for post-process cropping and zooming using analytical software.

\section{Analytical Techniques}

WSxM 4.0 software was used to analyze acquired data to produce figures and do quantitative analysis of the results. ${ }^{149}$ Files were imported into the software package and processed in a variety of ways for presentation and to remove scan artifacts. Line profiles of scanning data were commonly measured using 
the software. Flooding analysis was also performed in some cases to quantitatively determine feature sizes, which is particularly important for nanocomposites.

In AFM topography images, it is common for the measured surface to have some long range curvature that is an artifact of the scanning process. To account for this, analytical software packages such as WSxM offer a variety of "flattening" options to more accurately present the topography of the actual surface. WSxM provides three options to flatten the results: "Simple Flatten"; "Flatten discarding regions"; and "Flatten using paths". Each approach has applications depending on the surface being measured. For uniform films that do not have any particulate on the surface, a simple flatten with parabolic subtraction along the scanning rows to account for higher order artifacts was generally sufficient. If irregular particulate was present, discarding regions allows the user to selectively remove that region from the flattening algorithm and treat the rest of the scan area as a flat surface. This was employed in the case of samples with particulate on the surface or in some unpatterned nanocomposite films. Using paths for the flattening process was particularly important for the regular arrays of islands and nanopillars that were formed for the patterned nanocomposites. The nature of periodic arrays of features makes other flattening techniques difficult, as the rows of pillars will confuse the algorithm and make the unpatterned regions appear irregular. In these cases, a series of paths was drawn between the features in areas that were expected to be flat on the substrate surface or in the matrix of the nanocomposite. A parabolic subtraction was then performed. An example of this flattening process is shown in Figure 4-8. 


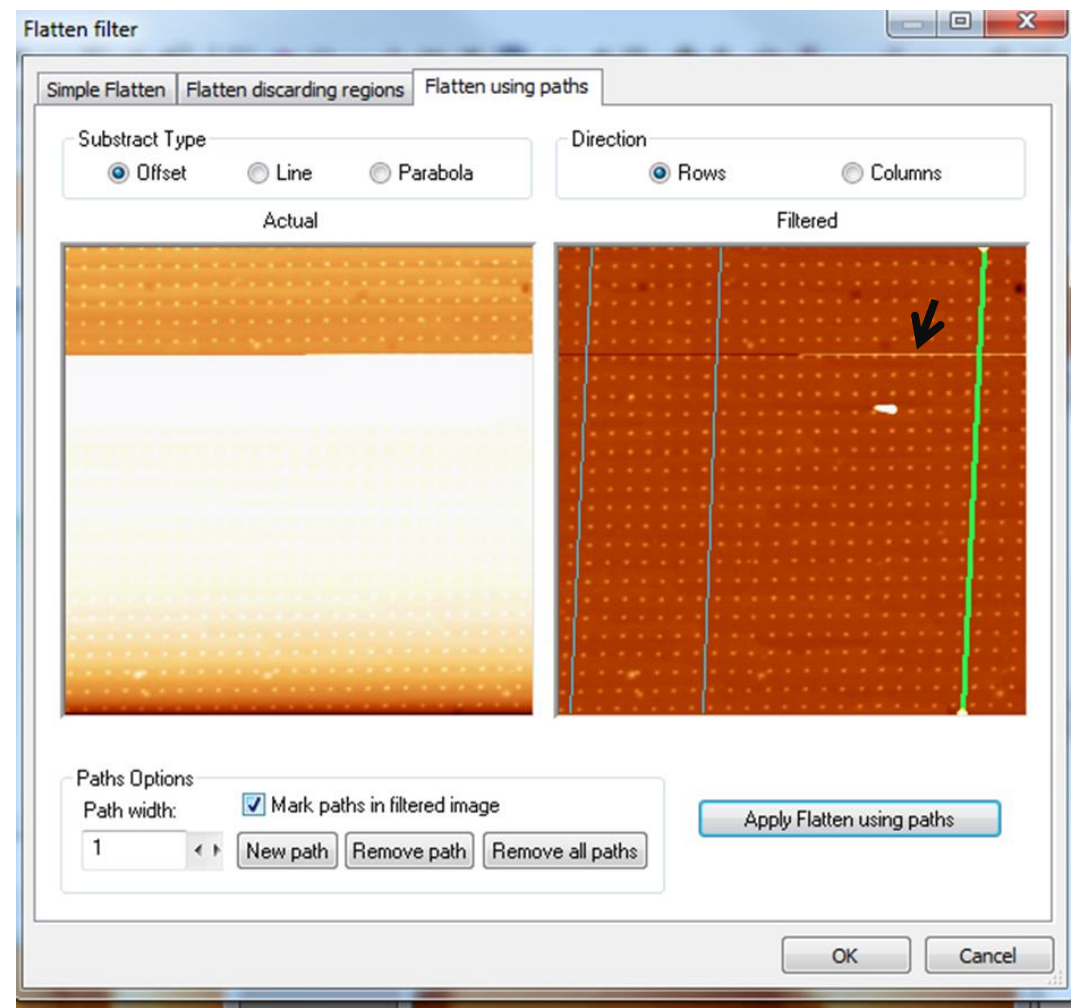

Figure 4-8. Screencapture from WSxM software showing the flattening process for an array of CoFe2O4 islands on the surface of SrTiO3. Note the bad scan line approximately $20 \%$ from the top in the filtered image marked with an arrow.

In some cases, a single scan line of an AFM image is disturbed do a surface feature or control system error. An example of this can be seen in the filtered image of Figure 4-8, denoted by an arrow. These scanning artifacts must be corrected to perform good analysis of the data. When present, the Remove Lines feature of WSxM was used to average any bad lines with the scan lines above and below the artifact. This provides a simple solution in most cases with no need for further corrections.

Flooding analysis is a key analytical technique to quantitatively study surface morphology. In this approach, all heights below a certain value are set to zero and only features above that height remain in the image. WSxM provides statistics which show the number of features above the flooding threshold, the fractional area coverage of these features in the overall scan, and the volume of features above the surface. Using this information and a priori knowledge of the sample, it is generally possible to estimate 
average lateral dimensions and heights of features in a nanocomposite. An example of a flooded AFM scan is shown in Figure 4-9.

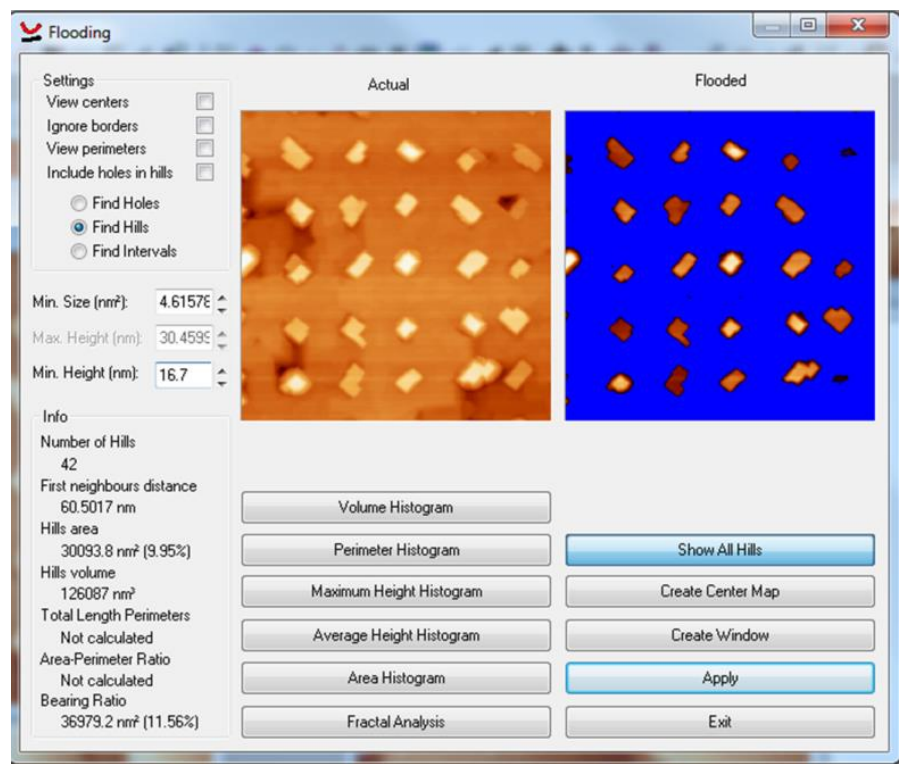

Figure 4-9. Screencapture from WSxM software showing the flooding process for an patterned nanocomposite with $\mathrm{CoFe}_{2} \mathrm{O}_{4}$ pillars in a $\mathrm{BiFeO}_{3}$ matrix. 


\section{Epitaxial Complex Oxide Films Grown by PED}

To benchmark the growth of the relevant materials for the $\mathrm{CoFe}_{2} \mathrm{O}_{4}-\mathrm{BiFeO}_{3}$ nanocomposites examined in the upcoming chapters, uniform epitaxial $\mathrm{CoFe}_{2} \mathrm{O}_{4}(\mathrm{CFO})$ and $\mathrm{BiFeO}_{3}(\mathrm{BFO})$ films were grown via pulsed electron deposition (PED). The microstructure and strain conditions of these films were examined via atomic force microscopy (AFM) and x-ray diffraction (XRD). These experiments enabled the characterization of the ferrimagnetic properties of $\mathrm{CFO}$, in particular the magnetic anisotropy. To enable measurements of the ferroelectric properties of $\mathrm{BFO}$ with a bottom electrode, $\mathrm{La}_{0.72} \mathrm{Sr}_{0.28} \mathrm{MnO}_{3}$ (LSMO) films were grown and their epitaxial growth modes were characterized. Finally, a bilayer structure of BFO on top of LSMO was grown and examined via piezoresponse force microscopy (PFM). For all samples, cation stoichiometry was inferred by examining the lattice parameter measured through x-ray diffraction. If volume in the unit cell of the film was conserved, it was assumed that the film was sufficiently stoichiometric for the purposes of this work.

\section{1. $\mathrm{CoFe}_{2} \mathrm{O}_{4}$ Films}

\section{Mg0 Substrates}

Films grown on $\mathrm{MgO}$ exhibited in-plane tensile strain due to the lattice mismatch with the substrate. The calculated lattice mismatch is given by:

$$
f=\frac{2\left(a_{f}-a_{s}\right)}{a_{f}+a_{s}} \sim \frac{a_{f}-a_{s}}{a_{f}}
$$

where $f$ is the dimensionless lattice mismatch, $a_{s}$ is the substrate lattice parameter, and $a_{f}$ is the bulk lattice parameter of the film. ${ }^{35}$ For CFO films grown on $\mathrm{MgO}$ (lattice parameter, $a=4.212 \AA$ ), the lattice mismatch is approximately $0.5 \%$, Out-of-plane XRD scans of the MgO (002) peak and CFO (004) peak are shown in Figure 5-1. These curves possess the thickness fringes that result from highly epitaxial layerby-layer growth. The presence of thickness fringes in an XRD scan is unusual for this system and is not seen in other works, indicating extremely high quality films. ${ }^{45,46,150}$ The in-plane lattice parameters for 
each film were determined by performing a reciprocal space map about the $\mathrm{MgO}(224)$ and $\mathrm{CFO}(448)$ peaks. These reciprocal space maps are shown in Figure 5-2. The extracted CFO lattice parameters and other results of these measurements are shown in Table 5-1. The $20 \mathrm{~nm}$ thick sample shows complete coherence with the substrate. The full-width at half-maximum (FWHM) of the rocking curves is less than $0.02^{\circ}$ in all cases, while the substrate FWHM was only slightly better, with values between $0.01^{\circ}$ and $0.015^{\circ}$. AFM analysis revealed that the films grew layer-by-layer on the $\mathrm{MgO}$ substrate and that the step edges were visible at thicknesses as large as $40 \mathrm{~nm}$. Step-edges were $\sim 8 \AA$ in height, corresponding to a single CFO unit cell. These scans, with corresponding MFM phase images, are shown in Figure 5-3. As misfit dislocations begin to form in thicker films, the surface morphology changes due to the strain fields present near the dislocations. It has been shown in other materials systems that islands form on the surface after dislocations have nucleated and are visible in AFM scans. ${ }^{151}$ The presence of islands is thus an indication that the film has begun to relax and dislocations have formed.

\begin{tabular}{|c|c|c|c|c|c|c|}
\hline Sample & $\begin{array}{l}\text { Out-of-plane } \\
\text { lattice } \\
\text { parameter, } c \\
(\AA) \\
(\AA)\end{array}$ & $\begin{array}{l}\text { In-plane } \\
\text { lattice } \\
\text { parameter, } \\
\text { a }(\AA)\end{array}$ & $\begin{array}{l}\text { FWHM of } \\
\text { CFO }(004) \\
\text { rocking } \\
\text { curve }\left({ }^{\circ}\right)\end{array}$ & $\begin{array}{l}\text { Saturation } \\
\text { magnetization, } \\
M_{s}(\text { emu/cc })\end{array}$ & $\begin{array}{l}\text { Coercivity } \\
\text { along out- } \\
\text { of-plane } \\
\text { axis (kOe) }\end{array}$ & $\begin{array}{l}\text { Squareness, } \\
\left(M_{r} / M_{s}\right)\end{array}$ \\
\hline $20 \mathrm{~nm}$ & $8.331 \pm 0.002$ & $8.410 \pm 0.004$ & $\begin{array}{l}0.0190 \pm 0.00 \\
08\end{array}$ & 170 & 1.3 & 0.39 \\
\hline $40 \mathrm{~nm}$ & $8.341 \pm 0.002$ & $8.401 \pm 0.004$ & $\begin{array}{l}0.0090 \pm 0.00 \\
15\end{array}$ & 180 & 7.4 & 0.41 \\
\hline $82 \mathrm{~nm}$ & $8.346 \pm 0.002$ & $8.400 \pm 0.004$ & $\begin{array}{l}0.0166 \pm 0.00 \\
07\end{array}$ & 235 & 7.9 & 0.43 \\
\hline
\end{tabular}

Table 5-1. Crystallographic and magnetic data for films in the study. 


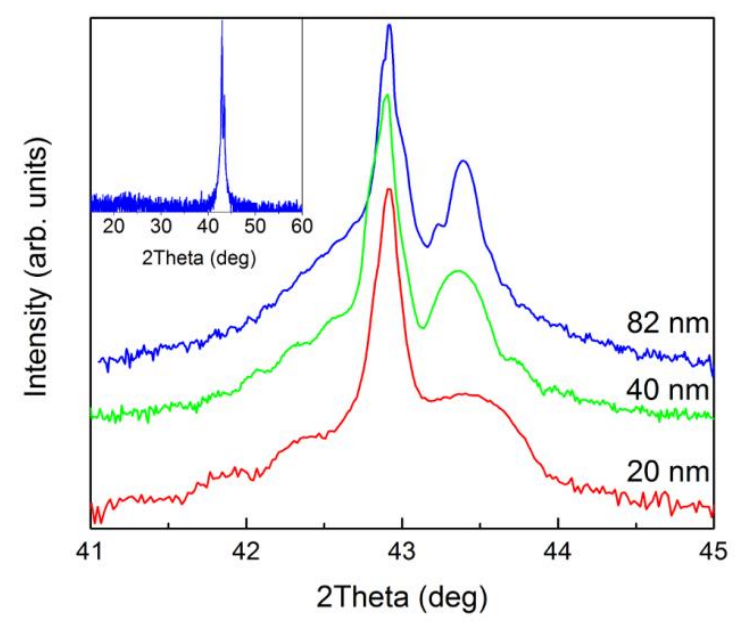

Figure 5-1. X-ray diffraction patterns of $\mathrm{MgO}(002)$ (left peak) and $\mathrm{CoFe}_{2} \mathrm{O}_{4}(004)$ (right peak) for films with thicknesses of $20 \mathrm{~nm}, 40 \mathrm{~nm}$, and $82 \mathrm{~nm}$ showing thickness fringes in all three cases. (Inset) Wide range scan for thickest film showing that no secondary phases are present.

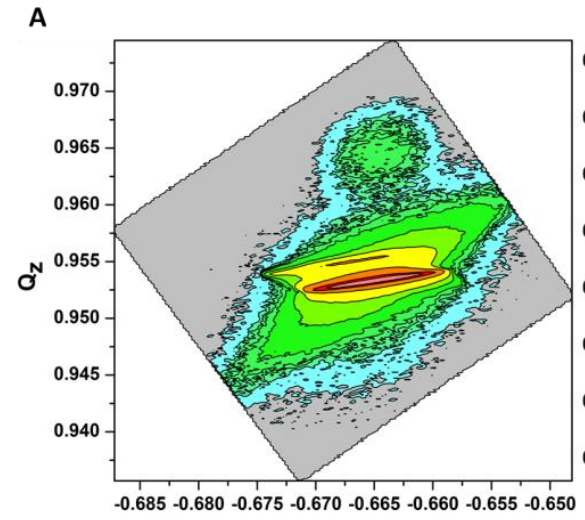

$Q_{x}$
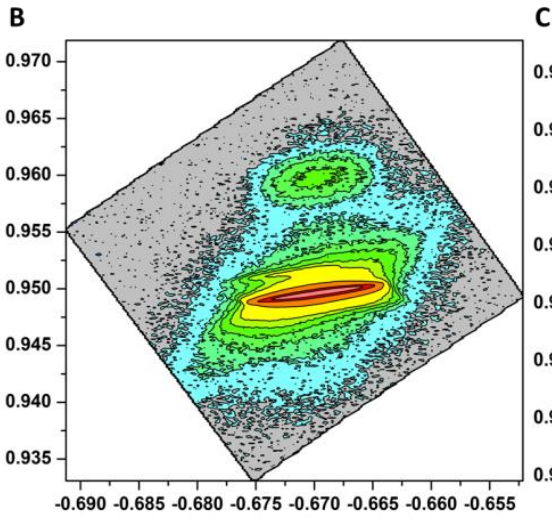

$Q_{x}$

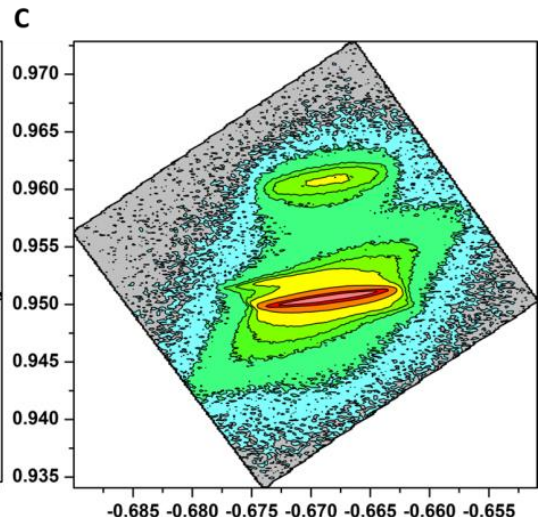

$Q_{\mathrm{x}}$

Figure 5-2. Reciprocal space maps about the MgO (224) peak (bottom peak) for CFO films (top peak) with thicknesses: (A) $20 \mathrm{~nm},(B) 40 \mathrm{~nm}$, and (C) $82 \mathrm{~nm}$. Units of both $Q_{x}$ and $Q_{z}$ are $\AA^{-1}$, with $Q_{x}$ representing the [110] direction and $Q_{z}$ representing the [001] direction. 


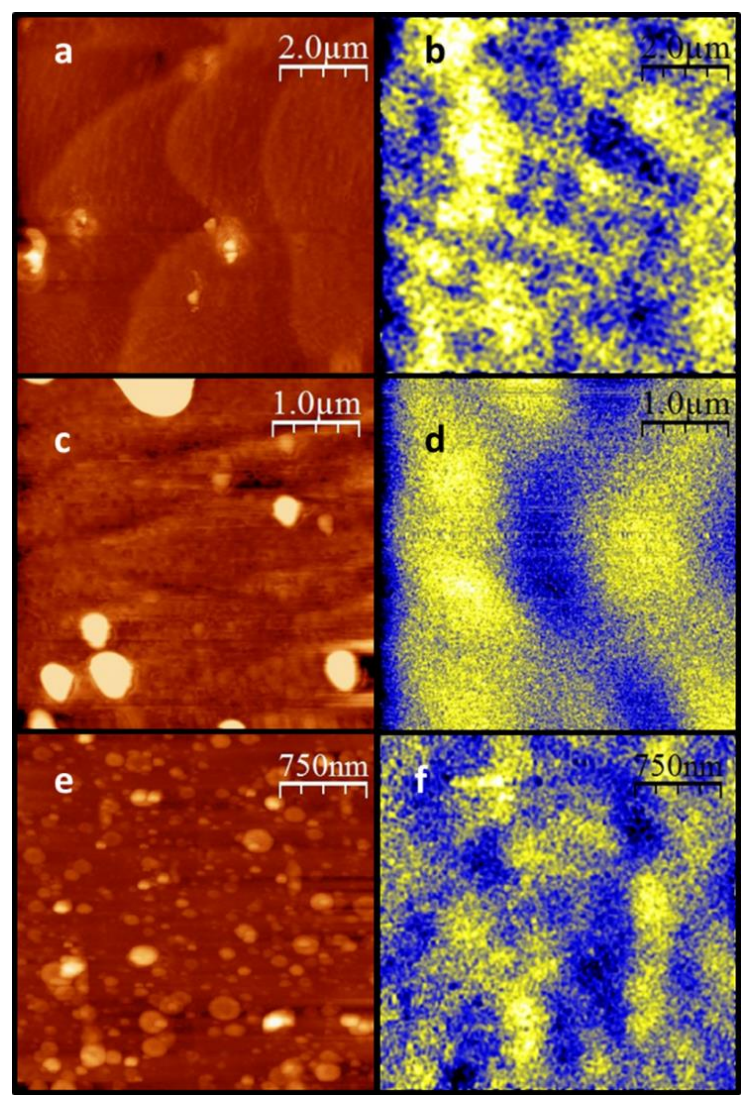

Figure 5-3. (a) AFM topography of $20 \mathrm{~nm}$ film; (b) MFM phase corresponding to (a); (c) AFM topography of $40 \mathrm{~nm}$ film; (d) MFM phase corresponding to (c); (e) AFM topography of $82 \mathrm{~nm}$ film; (f) MFM phase corresponding to $(\mathrm{e})$. Note that the scale bars vary between images. Image scales were chosen to include a comparable number of domains in each image.

Misfit dislocations are expected to form in CFO with a Burgers vector of $\frac{1}{4}[110]$, with a magnitude of $1.48 \AA$ for films grown on $\mathrm{MgO} .{ }^{152}$ By adapting the Matthews-Blakeslee formula, it is possible to estimate the critical thickness at which a misfit dislocation form in the film. ${ }^{36,153}$ Following the work of Kale et al., who used the same approach for $\mathrm{Fe}_{3} \mathrm{O}_{4}$ films on $\mathrm{MgO}$, and assumed a Poisson's ratio of 0.28, the critical thickness for the formation of dislocations in the system is approximately $35 \mathrm{~nm} .{ }^{154}$ Films with thicknesses of less than $35 \mathrm{~nm}$ are expected to be fully coherent with the $\mathrm{MgO}$ substrate, while thicker films should have some misfit dislocations. This result is in good agreement with the interpretation of the AFM and x-ray results, in that there are no islands visible on the surface for the $20 \mathrm{~nm}$ film and the 
reciprocal space map shows that the film is coherent with the substrate in-plane. In the $40 \mathrm{~nm}$ film, the islands protrude $\sim 5 \mathrm{~nm}$ above the surface and are reasonably scarce, while the reciprocal space map shows slight relaxation. In the $82 \mathrm{~nm}$ film, the islands dominate the morphology and are larger than those in the $40 \mathrm{~nm}$ film, indicating a larger dislocation density and additional film relaxation, which is confirmed in the reciprocal space map.

MFM measurements were performed on all three samples after demagnetizing the samples in a perpendicular magnetic field. Topography and magnetic phase images are shown in Figure 5-3. For the two thinner films (Figure 5-3(a-d)), the domains were stripe-like in nature, indicating $180^{\circ}$ domain walls and perpendicular anisotropy. ${ }^{131}$ The presence of stripe-like domains in the system was predicted but not shown by Yanagihara et al., who grew films with similar epitaxial quality and hysteretic properties to those shown in this work. ${ }^{47}$ Domain width decreased with thickness, which agrees with expectations given that the magnetic moment per unit area will increase with thickness. $180^{\circ}$ domain walls were also present in the thickest film. However, a more complex domain structure was found, most likely due to the effects of surface morphology and the high density of misfit dislocations.

Hysteresis loops for both in-plane and out-of-plane directions are shown in Figure 5-4, with coercivity and remanence data presented in Table 5-1. The effects of shape anisotropy have been removed from the figures, by setting $H=H_{\text {applied }}-4 \pi M$ in the case of perpendicular measurements. The in-plane loops exhibit minimal hysteresis, indicating that the magnetic easy axis is perpendicular to the plane of the film. 

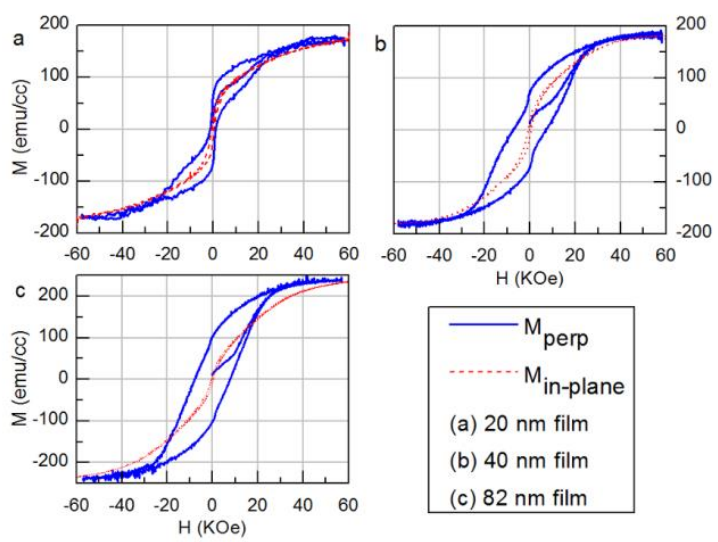

Figure 5-4. Perpendicular (solid, blue) and in-plane (dotted, red) M vs. H hysteresis loops for all three films: (a) $20 \mathrm{~nm}$ film; (b) $40 \mathrm{~nm}$ film; (c) $82 \mathrm{~nm}$ film.

We propose a model for the out-of-plane demagnetization process in the thinnest sample, as the field is swept from the positive to the negative saturation fields. At small positive fields, domain walls nucleate in the film, reducing the net magnetization. The nucleation of domain walls is most likely due to the presence of antiphase boundaries in the film. Previous work in $\mathrm{Fe}_{3} \mathrm{O}_{4}$ and $\mathrm{CFO}$ films grown on $\mathrm{MgO}$ showed that the inverse spinel structure naturally forms antiphase boundaries at the film-substrate interface due to the asymmetry between the oxygen sublattices in each system. ${ }^{155,156}$ The reduction in remanent magnetization for CFO grown in other systems has been attributed to antiphase boundaries as well. ${ }^{157,158}$ Thus, the presence of domain walls accounts for the relatively low remanence in a film with large perpendicular anisotropy. As $H$ becomes negative and passes the coercive field, the domain walls travel unimpeded through the film since there are few defects to pin their motion. Finally, the moment saturates in the negative direction at a saturation field of approximately $-40 \mathrm{kOe}$. The large saturation field is required to completely annihilate the domain walls.

The increase in coercive fields from the $20 \mathrm{~nm}$ sample to the thicker samples can be attributed to the presence of misfit dislocations at the interface between CFO and $\mathrm{MgO}$. In the case of the $20 \mathrm{~nm}$ film, few or no misfit dislocations are present, as seen in the AFM scan in Fig. 3a. Hence, domain wall pinning was 
not pronounced for the thinnest film and the coercivity is relatively low $(1.3 \mathrm{kOe})$. In the $40 \mathrm{~nm}$ film, the defect density is low, allowing the domain walls to move finite distances before impinging on a dislocation. This is shown in the initial drop-off in the moment at small negative fields (Fig. 4b). In the case of the thickest film, the defect density is high enough that the walls traverse a negligible distance before impinging on a dislocation. Hence, there is a smooth decrease in moment from remanence to the coercive field (Fig. 4c). An increase in the saturation magnetization is also observed in the thicker films. Given that antiphase boundaries reduce the magnetic moment at the substrate interface, it is reasonable to expect that thicker films will have larger saturation moments since the contribution of the interface to the overall moment is smaller. ${ }^{159}$

\section{2. $\mathrm{SrTiO}_{3} \mathrm{Substrates}$}

Unlike MgO, STO shares a relatively poor lattice mismatch, with a lattice parameter of $3.905 \AA$. For CFO films on STO, if one divides the CFO lattice parameter by two to account for the unit cell doubling, the lattice mismatch is equal to $7.0 \%$. Given such a large lattice mismatch, we would expect CFO to grow under the Volmer-Weber island growth mode, which has been demonstrated elsewhere. ${ }^{49}$ Through the formation of islands, the film-substrate interface is incoherent and strain is almost entirely relaxed in the film. Optimized CFO films were grown at $515^{\circ} \mathrm{C}$ on STO substrates at $12 \mathrm{mTorr} \mathrm{O}_{2}$ pressure, with $9 \mathrm{kV}$ gun voltage and $8 \mathrm{~Hz}$ pulse rate. The thickness of the optimized film was found to be $11 \mathrm{~nm}$ based on $\mathrm{x}$ ray reflectivity analysis. An AFM image of the surface topography is shown in Figure 5(a), demonstrating uniform islands covering the surface. A representative film grown at $12 \mathrm{kV}$ gun voltage with $17 \mathrm{mTorr}_{2}$ pressure is also shown in Figure 5-5(b). 

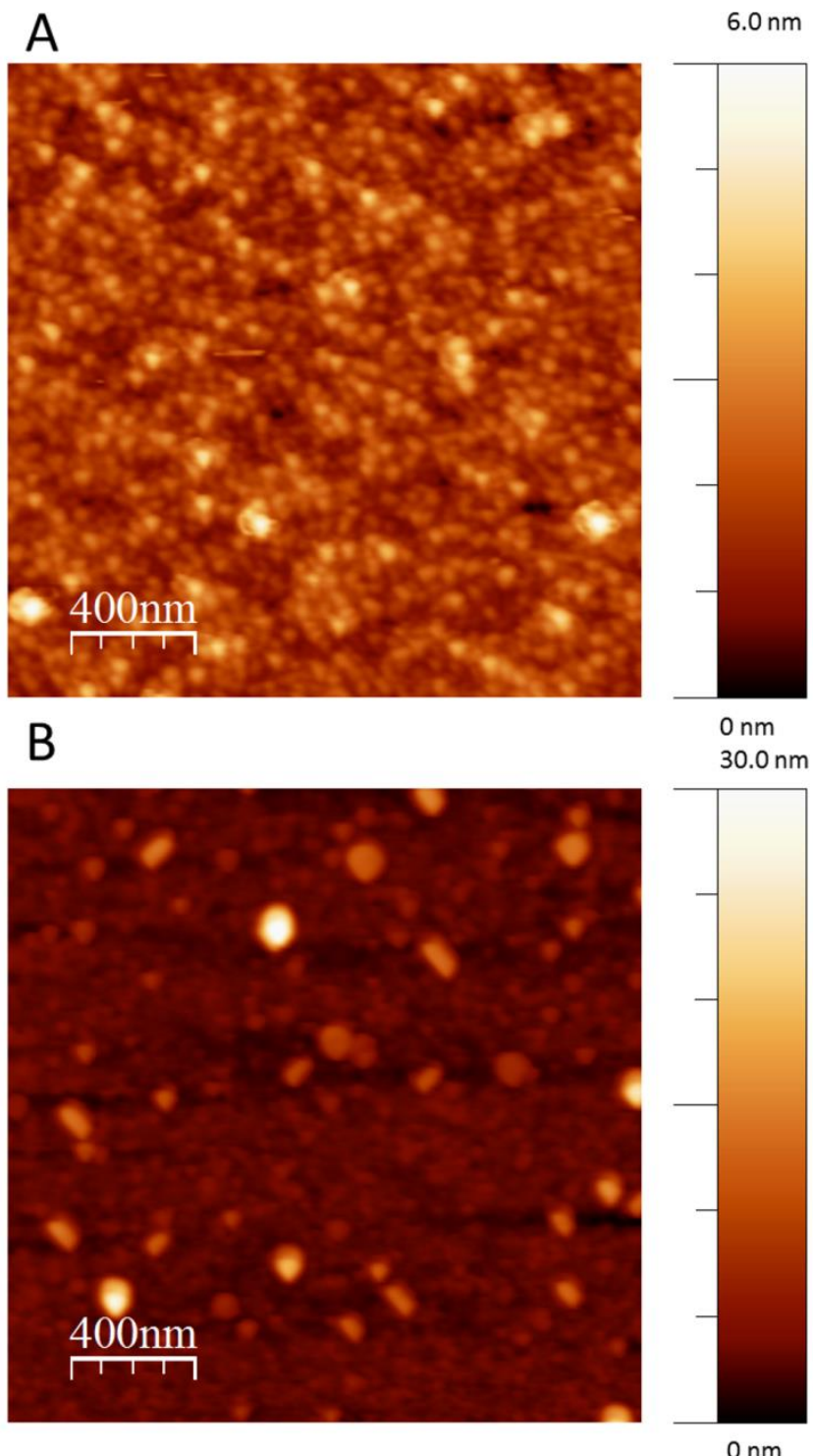

Figure 5-5. Atomic force microscopy topography scans of $\mathrm{CoFe}_{2} \mathrm{O}_{4}$ films grown at (a) $9 \mathrm{kV}$ and (b) $12 \mathrm{kV}$, showing similar island growth modes but different particulate densities. Note the difference in scale between the images.

We note that higher gun voltages produce films with more particulate on the surface, which has been demonstrated previously. In the previous work, Nistor et al. (Ref. 18) demonstrated that total pulse energy, which is equal to $1 / 2 C V^{2}$, where $C$ is the capacitance of the electron gun and $V$ is the discharge voltage, affects the density of particulates on the surface of $\mathrm{ZnO}$ films. ${ }^{160}$ They were able to reduce, but not fully eliminate particulate in the films. Our optimized CFO films do show some particulate over larger 
scales, though the density is significantly less than in the Nistor work. The low particulate density in these films is particularly important for applications that involve the patterning of the films, such as the directed self-assembly of CFO-BFO nanocomposites, which will be discussed in Chapter $7 .^{100}$

\section{2. $\mathrm{La}_{0.72} \mathrm{Sr}_{0.28} \mathrm{MnO}_{3}$ Films}

$\mathrm{La}_{0.72} \mathrm{Sr}_{0.28} \mathrm{MnO}_{3}$ films were grown on both $\mathrm{STO}$ and $\mathrm{LAO}$ as a means of developing a bottom electrode for ferroelectric BFO films. LSMO has a bulk lattice parameter of $3.895 \AA$, giving it a lattice mismatch with STO of $-0.3 \%$ and with LAO of $2.7 \%$. Such a small lattice mismatch with STO should readily promote layer-by-layer growth, while LSMO grown on LAO could be expected to fall in the Stranski-Krastanov (S-K) growth regime. ${ }^{31}$ In S-K growth, a highly strained film initially begins to grow layer-by-layer, as it uniformly wets the surface. As the thickness increases, the film begins to relax through the formation of islands on the film surface, which relieve strain energy by allowing the lattice to expand or contract within the island.

LSMO films of thicknesses $2.1 \mathrm{~nm}$ and $4.4 \mathrm{~nm}$ were deposited simultaneously onto STO and LAO substrates mounted side-by-side on the sample stage to compare the morphology that results from the different strain conditions and from relaxation effects as thickness increases. The films were deposited in a $70 \% \mathrm{O}_{2}: 30 \%$ Ar mixture with chamber pressure of $15 \mathrm{mTorr}$ and a set temperature of $577^{\circ} \mathrm{C}$. The gun voltage was set to the minimum $9 \mathrm{kV}$ with a $5 \mathrm{~Hz}$ pulse rate. X-ray reflectivity of the film grown on STO indicates an LSMO film thickness of $4.4 \mathrm{~nm}$, which equates to a deposition rate of $0.002 \AA /$ pulse or $0.7 \AA / m i n$. AFM topography measurements for the films grown on LAO are shown in Figure 5-6. Similar measurements for films simultaneously grown on STO are shown in Figure 5-7. In the case of the films grown on STO, a smooth, uniform surface is present with a small amount of particulate deposited from the target. In the case of LSMO films, a low voltage is required to reduce particulate emission, while relatively high oxygen background pressures are needed to fully oxidize the film, which enhances the magnetic and electrical properties. Thus, a tradeoff must be made to grow the films very slowly in order to achieve the desired morphology and physical properties. The films grown on LAO show similar 
particulate density to those grown on STO. The $2.1 \mathrm{~nm}$ film shows layer-by-layer growth and a uniform surface, while islands dot the surface in the case of the thicker $4.4 \mathrm{~nm}$ film (Figure 5-6(c)). A crosssection of three of the islands is shown in Figure 5-6(d). The islands rise approximately $1 \mathrm{~nm}$ above the surface at an angle of 4 to $6^{\circ}$. Thus, it seems safe to conclude that the islands are an example of StranskiKrastanov growth.
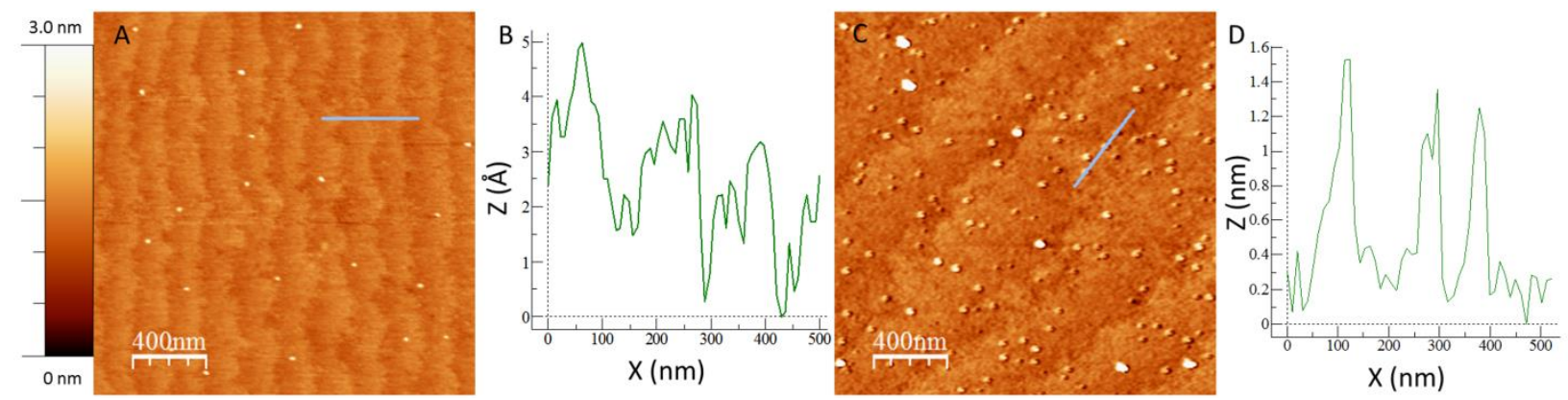

Figure 5-6. Atomic force microscopy topography scans of $\mathrm{La}_{0.72} \mathrm{Sr}_{0.28} \mathrm{MnO}_{3}$ films grown on (a) $\mathrm{SrTiO}_{3}$ and (b) $\mathrm{LaAlO}_{3}$ showing Frank-van der Merwe and Stranski-Krastanov growth modes. (c) A cross-sectional line scan along the gray line shown in (b) showing the height of islands above the film surface.
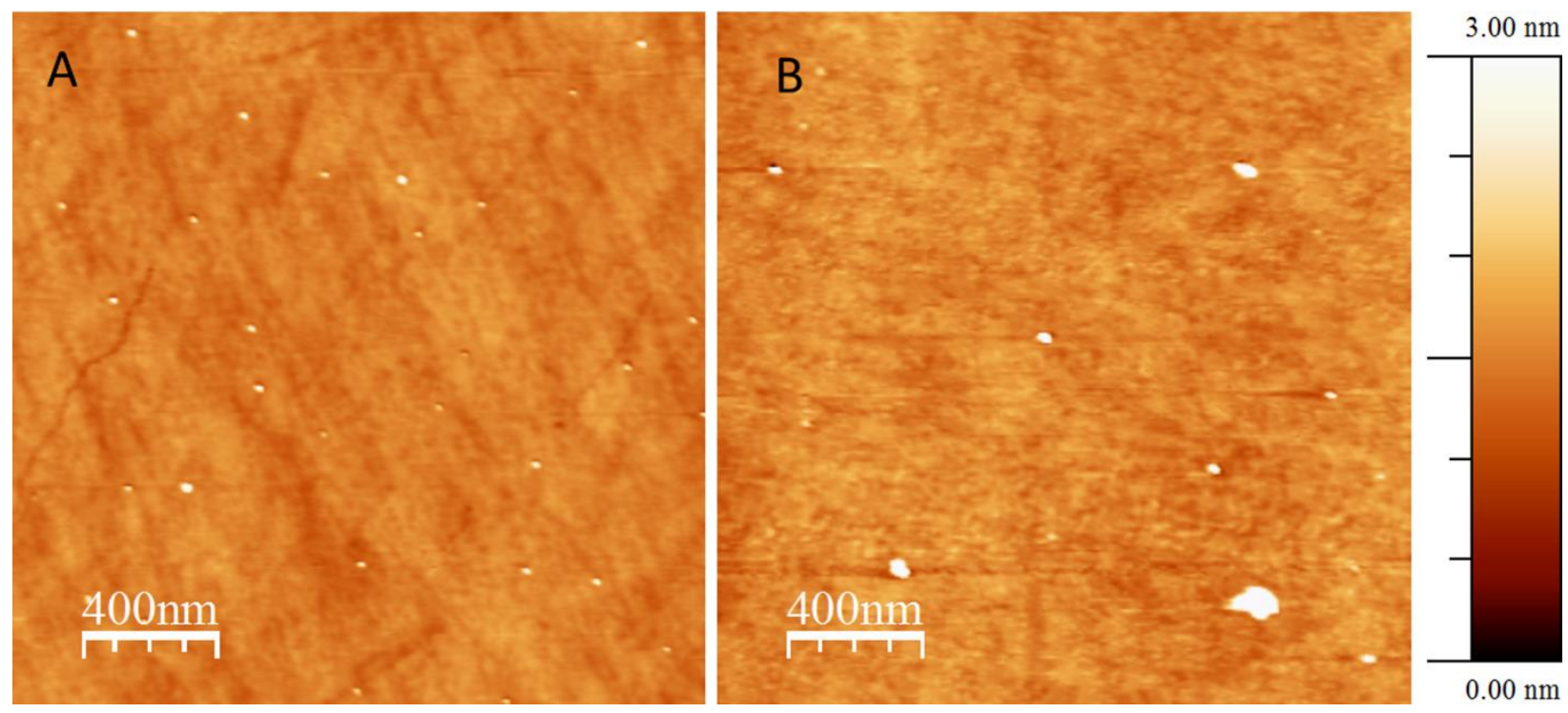

Figure 5-7. Atomic force microscope topography images of (A) $2.1 \mathrm{~nm}$ thick LSMO film grown on STO substrate; (B) $4.4 \mathrm{~nm}$ thick LSMO film grown on STO substrate. 


\section{3. $\mathrm{BiFeO}_{3}$ Films}

\section{Structural Properties of Films}

Rhombohedral-phase BFO is commonly grown on STO, since the pseudocubic lattice parameter of $3.96 \AA$ provides a lattice misfit of only $1.4 \% .{ }^{161}$ Such a small misfit should allow for layer-by-layer (Frank-van der Merwe) growth. Meanwhile, $\mathrm{LaAlO}_{3}(\mathrm{LAO})$, with a pseudocubic lattice parameter of 3.79 A shares a relatively large misfit with the rhombohedral BFO of $4.4 \% .{ }^{35}$ However, recent results have demonstrated that when significant in-plane compressive strains are applied — as is the case with LAOthat an alternative tetragonal phase of BFO is stabilized. ${ }^{65-67}$ This phase has an in-plane lattice parameter very close to that of LAO, with theoretical models predicting values of 3.67 to $3.73 \AA$, with out-of-plane lattice parameter 1.2 to 1.3 times larger than the in-plane lattice parameter. ${ }^{162,163}$ Layer-by-layer growth of these films on LAO was demonstrated using PLD with clear step edges visible in AFM topography scans. ${ }^{164}$ BFO films were deposited separately onto STO and LAO substrates in a mixture of $70 \% \mathrm{O}_{2}$ and $30 \%$ Ar gas at a chamber pressure of 12 mTorr and substrate temperature of $577^{\circ} \mathrm{C}$. The thickness of the film on STO was calculated to be $5.9 \mathrm{~nm}$ based on $\mathrm{x}$-ray reflectivity measurements and the thickness of the film on LAO was calculated to be $9.3 \mathrm{~nm}$ based on Kiessig fringe spacing from diffraction data. A $\mathrm{Bi}_{1.15} \mathrm{FeO}_{3}$ target was ablated with $12 \mathrm{kV}$ gun voltage and $5 \mathrm{~Hz}$ pulse rate. The target with excess $\mathrm{Bi}$ was chosen due to the high reactivity and vapor pressure of $\mathrm{Bi}$ atoms, which leads to desorption of $\mathrm{Bi}$ ions from the sample during growth.

We have achieved comparable layer-by-layer growth of BFO on both STO (rhombohedral phase) and LAO (tetragonal phase). AFM topography images are shown in Figure 5-8, along with x-ray diffraction for both films showing Kiessig fringes, which indicates a high quality interface between film and substrate. ${ }^{164}$ We see clear indication of step-edges in both films, indicating layer-by-layer growth. In the case of the film grown on STO, however, we see that islands with unit cell height have formed within the terrace. In the case of the film on LAO, there is some evidence of step-bunching, where step edges merge together such that some terrace steps are multiple unit cells in height. For both films, this phenomenon 
can most likely be traced to the variation in terrace length between the substrates. Other work has shown that terrace length plays a significant role in determining whether layer-by-layer growth is achieved via step-flow growth, island formation or step-bunching. ${ }^{32}$
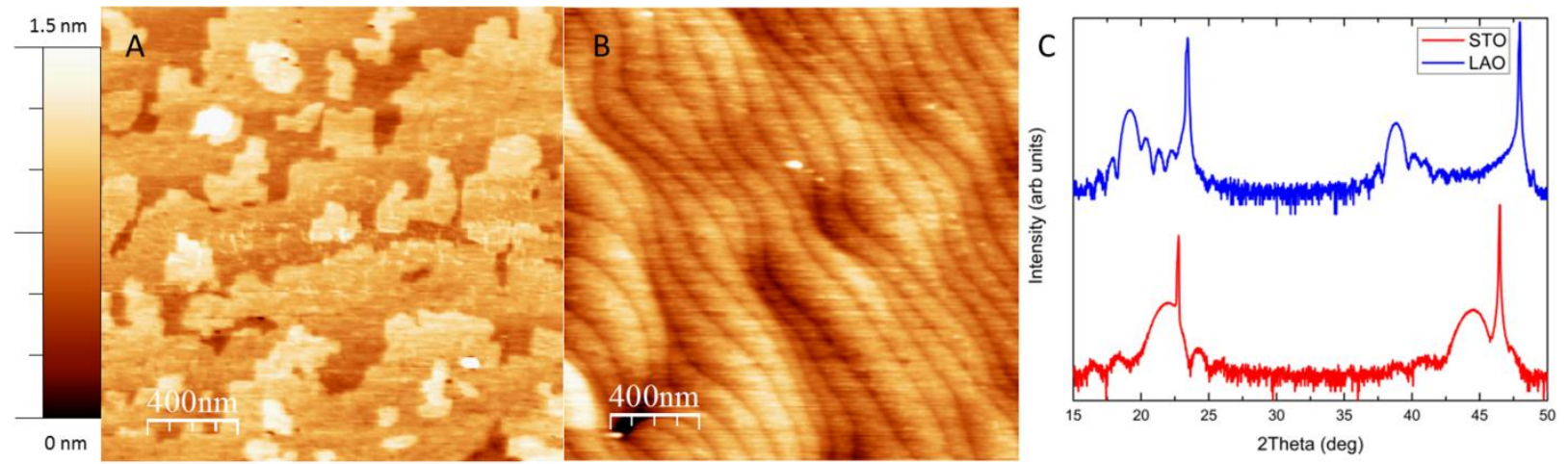

Figure 5-8. Atomic force microscopy topography scans of $\mathrm{BiFeO}_{3}$ films grown on (a) $\mathrm{SrTiO}_{3}$ and (b) $\mathrm{LaAlO}_{3}$. (c) $\mathrm{X}$-ray diffraction measurement of the (001) and (002) substrate and film peaks for both films showing Kiessig fringes.

\section{Ferroelectric Properties of $\mathrm{BiFeO}_{3}$ Films}

To test the ferroelectric quality of the BFO films grown via PED, a bilayer structure was grown on an STO substrate, producing a BFO/LSMO/STO structure. The thicknesses for each layer of the film were determined using x-ray reflectivity and found to be approximately $10.9 \mathrm{~nm}$ for BFO and $4.6 \mathrm{~nm}$ for LSMO. X-ray diffraction measurements were performed and are shown in Figure 5-9(a). The results show clear BFO (001) and (002) pseudocubic peaks with Kiessig fringes, but the LSMO peak is shadowed by the overlapping STO substrate peak. 

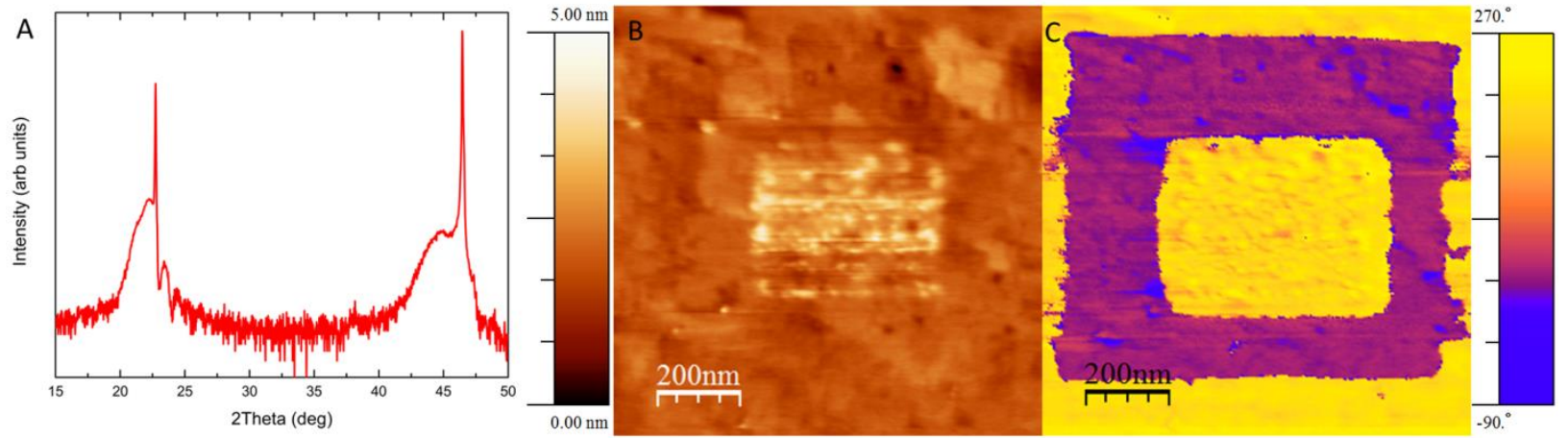

Figure 5-9. (a) X-ray diffraction measurement of $\mathrm{BiFeO}_{3}-\mathrm{La}_{0.72} \mathrm{Sr}_{0.28} \mathrm{MnO}_{3}$ bilayer grown on $\mathrm{SrTiO}_{3}$; (b) Piezoresponse force microscopy (PFM) topography scan of sample; (c) PFM out-of-plane phase scan of sample showing switched regions.

Dual AC resonance-tracking piezoresponse force microscopy (DART PFM) measurements ${ }^{147}$ were performed on the film by making a contact to the conductive LSMO bottom layer with conductive silver paste. ${ }^{165}$ A $750 \mathrm{~nm}$ square region was initially scanned with a positive bias on the tip of $3 \mathrm{~V}$ to switch the out-of-plane polarization away from the single domain as-grown state. A second $375 \mathrm{~nm}$ scan was performed inside of the switched region with a $-3 \mathrm{~V}$ tip bias to switch that region back to the as-grown out-of-plane polarization. The topography and phase PFM images measured after this switching process are shown in Figure 5-9(b-c). We note that there is clear evidence of $180^{\circ}$ switching in the out-of-plane polarization in Figure 5-9(c). A change in height within the $375 \mathrm{~nm}$ scan of approximately $1 \mathrm{~nm}$ can also be seen. This morphology change is attributable to chemical interactions of the surface with atmospheric water vapor when the biased tip is in contact with the region, which can produce adsorbates on the surface. ${ }^{166}$ Surface roughness is calculated to be $\sim 2 \AA$ (Root Mean Square roughness) in the regions unaffected by the adsorbates. These results demonstrate that the achievement of an insulating and ferroelectric BFO film on top of a conductive LSMO film, while maintaining a smooth, uniform surface morphology. Similar results have been observed in $\mathrm{BFO}$ films grown on $\mathrm{SrRuO}_{3}$ bottom electrodes in our research group. ${ }^{167}$ 


\section{Film Growth Kinetics}

Competition between nucleation in the formation of islands and crystal growth in the case of layerby-layer growth is a key governing feature in epitaxial film growth. The growth mode of a thin film is determined by the interfacial energy between the film and substrate, the surface energy of the film and the surface energy of the substrate. ${ }^{34}$ If one assumes that the atoms in the vapor are in equilibrium with those on the film surface it is possible to write the criteria for layer-by-layer growth as:

$\Delta \gamma=\gamma_{F}+\gamma_{i}-\gamma_{S} \leq 0$

where $\gamma_{F}$ is the surface energy of the film, $\gamma_{i}$ is the interfacial energy between the film and substrate, and $\gamma_{S}$ is the substrate surface energy. The interfacial energy is governed by the chemical bonds between the substrate and film and the strain interactions due to lattice mismatch. In the case of Volmer-Weber island growth, the surface energy of the film or the interfacial energy between the materials is large enough that the film will not wet the surface and layer-by-layer growth is impossible and island nucleation occurs. Given the large lattice mismatch between CFO and STO, along with the fact that the minimum energy surface for spinel oxides is the $\langle 111\rangle$ surface rather than the $\langle 100\rangle,{ }^{168}$ it is not surprising to observe pure island growth. We have observed some temperature dependence in the morphology of CFO films grown on STO. For films grown at lower substrate temperatures, there is insufficient energy to promote island nucleation, resulting in smoother films that have lower crystalline quality. At temperatures higher than in this report, we see more strongly facetted islands due to the additional adatom thermal energy. In the case of BFO films grown on STO and LAO, we see layer-by-layer growth due to the low interfacial energy between film and substrate, since the lattice mismatch is low and both the film and substrate exhibit the same perovskite crystal structure. For LSMO on LAO, both film and substrate have the same perovskite structure, but the lattice mismatch is large enough that layer-by-layer growth cannot be maintained beyond $\sim 4 \mathrm{~nm}$. 
It is worthwhile to consider the kinetics of the growth that lead to the high quality films demonstrated in this work. We have shown that we can achieve all three epitaxial growth modes in a variety of complex oxide materials using the PED technique. Traditionally, the PED technique has been used to grow much thicker films than we have shown here using significantly higher deposition rates. In one work, YBCO films were grown at a variety of target to substrate distances and growth rates at the $9 \mathrm{~cm}$ distance used in our chamber were $0.36 \AA$ /pulse. ${ }^{112}$ By contrast, the LSMO films presented in this work were grown at a rate of $0.002 \AA$ /pulse, which is more than 150 times slower than the growth rate in the previous work. In Strikovski et al., the authors showed that for a deposition rate of $0.1 \AA$ /pulse, the plasma emitted from the target after ablation propagated a distance of approximately $8 \mathrm{~cm}$ in a chamber with gas pressure of 17 mTorr. ${ }^{120}$ At this distance, they argued that half of the emitted species from the target would have undergone a collision with the ambient gas molecules and no longer have velocity directed at the substrate. This was suggested to be the optimal growth condition, with adatom energies in the range of 2$8 \mathrm{eV}$, which is an order of magnitude greater than the typical energy range of $0.1-1 \mathrm{eV}$ assumed in molecular beam epitaxy. ${ }^{169}$ In this work, deposition rates that are roughly 50 times slower than in the work discussed in Strikovski et al. ${ }^{120}$, and have demonstrated layer-by-layer growth that was not achieved in the previous work. Thus, we believe that it is likely that the growth conditions used fully thermalize the adatoms, producing higher quality films. We have found that the morphology of the samples is degraded at substantially higher deposition rates, both from increased particulate emission at higher gun voltages and changes in the morphology of the film in the clean regions from unknown kinetic changes, further supporting our hypothesis.

In the case of LSMO and BFO films, the addition of Ar gas to the system proved important to optimizing the film quality. It has been noted that the highest energy ions emitted from a target via PED may have energies in the range of several $\mathrm{keV}$, which is somewhat higher than the most energetic ions emitted during the PLD process. ${ }^{120}$ Heavy adatoms, such as Bi and La, with energies greater than $100 \mathrm{eV}$ can readily induce sputtering in the film and damage the surface of the film, which could prevent layer- 
by-layer epitaxial growth. Given the complex interactions governing the plasma dynamics in the spark, it is difficult to make categorical claims regarding the effect that Ar gas might have on the pulse energy. However, the empirical evidence presented in this work suggests that the addition of Ar may lower the energy imparted to the target during the pulse, which could reduce the number of high energy ions emitted from the target that can damage the film surface. In complex oxide films grown via PLD, Ar is not generally used as a process gas since the $\mathrm{O}_{2}$ operating pressures are significantly higher ( 100 mTorr) than in PED. However, the requirements to form an electron spark dictate that operating pressures be held between roughly 5 and 20 mTorr. Thus, addition of an inert process gas such as Ar can counteract the lower operating pressures needed for PED. Further studies are needed to fully understand the origin of this effect.

\section{Summary}

In this chapter, the growth of a variety of different complex oxide films has been demonstrated via pulsed electron deposition (PED). $\mathrm{CoFe}_{2} \mathrm{O}_{4}$ (CFO) films have been grown on $\mathrm{MgO}$ substrates, demonstrating layer-by-layer growth and perpendicular magnetic anisotropy due to tensile in-plane strain. CFO films on $\mathrm{SrTiO}_{3}$ (STO) exhibit the Volmer-Weber island growth mode due to the significant lattice mismatch between CFO and STO. $\mathrm{La}_{0.72} \mathrm{Sr}_{0.28} \mathrm{MnO}_{3}$ (LSMO) films have been grown on both STO and $\mathrm{LaAlO}_{3}(\mathrm{LAO})$ substrates and their epitaxial growth modes have been characterized. Due to an excellent lattice match with STO, the LSMO films have an extremely smooth surface that suggests layer-by-layer growth. Moderate compressive strain from growth of LSMO on LAO produces the Stranski-Krastanov layer-then-island growth mode. Finally, BFO films were grown on STO and LAO, demonstrating layerby-layer growth in both cases with the expected rhombohedral to tetragonal phase transition for films grown with compressive strain on LAO. A bilayer structure of BFO with an LSMO bottom electrode demonstrated that the BFO film was ferroelectric, as expected.

The various epitaxial growth modes demonstrated here via PED represent a significant breakthrough for the growth technique. Previous results in the literature were for thick films with rough surfaces due to 
particulate deposition and high growth rates. In this work, by carefully calibrating the growth conditions, all three epitaxial growth modes have been demonstrated for films grown via PED. Such results indicate that PED may be competitive with the more commonly used pulsed laser deposition (PLD) in the coming years. A recent result has demonstrated similarly smooth LSMO films grown via PED, suggesting that the technique is gaining traction in the field. ${ }^{170}$ 


\section{Epitaxial $\mathrm{CoFe}_{2} \mathrm{O}_{4}-\mathrm{BiFeO}_{3} \mathrm{Nanocomposites}$}

To develop an understanding of the structural properties of $\mathrm{CoFe}_{2} \mathrm{O}_{4}(\mathrm{CFO})-\mathrm{BiFeO}_{3}(\mathrm{BFO})$

nanocomposites grown via pulsed electron deposition (PED), a series of nanocomposite films was grown and characterized, which were use to compare these properties to those grown via pulsed laser deposition (PLD). As discussed in Chapter 2, the origin of the magnetic anisotropy in these nanocomposite films was not fully understood due to the combined effects of magnetoelastic and shape anisotropies in the materials. For device applications in systems such as the Reconfigurable Array of Magnetic Automata (RAMA), the ability to predictably control the magnetic anisotropy of the CFO nanopillars is critical. ${ }^{3}$ Thus, a substantial portion of this work focused on determining the effects of elastic strain and pillar shape on the magnetic anisotropy of the nanopillars. Examination of the ferroelectric properties of the BFO matrix also proved fruitful, as intriguing in-plane domain wall pinning in the matrix was observed.

\section{Structural Characterization}

\section{X-Ray Analysis}

In the literature, most CFO-BFO nanocomposites had thicknesses of several hundred nm when grown via pulsed laser deposition. The aspect ratio of the pillars in films with this thickness would be expected to produce perpendicular magnetic shape anisotropy in the pillars. There are also commonly reports of out-of-plane compressive strain observed in the CFO pillars in these samples. ${ }^{79}$ To understand the effect of strain and shape in the pillars, a series of composite films with different thicknesses was prepared. By growing films with different thicknesses, one might expect the shape anisotropy of the pillars to vary while the magnetoelastic anisotropy was expected to remain constant.

Films with BFO matrix thickness of $13 \mathrm{~nm}, 43 \mathrm{~nm}$ and $150 \mathrm{~nm}$ were grown and examined in this study. X-ray diffraction (XRD) analysis was performed to determine the CFO strain conditions in each film. The results are summarized in Table 6-1. The in-plane lattice parameters were calculated by 
performing reciprocal space maps about STO (224), which shares an epitaxial match to the pseudo-cubic BFO (224) peak and the CFO (448) peak. A representative XRD spectrum, along with an atomic force microscopy (AFM) scan showing the surface topography is shown in Figure 6-1. The average pillar aspect ratios for each film were determined using AFM and scanning electron microscope (SEM)

measurements and are shown in Table 6-1 as well. For AFM measurements, flooding analysis was used to determine the number of pillars in a $5 \mu \mathrm{m}$ square region and the fraction of area covered by the pillars.

The average height of the pillar above the surface was also determined using this approach to improve the estimation of the aspect ratio. SEM measurements were used to corroborate the area coverage results for sample 1 and 3, which had greater pillar area coverage, making AFM area analysis more difficult. A visual representation of the strain conditions in the samples is shown in Figure 6-1(c).

\begin{tabular}{|c|c|c|c|c|c|c|c|}
\hline Sample & $\begin{array}{l}\text { BFO } \\
\text { matrix } \\
\text { thickness, } \\
(\mathrm{nm})\end{array}$ & $\begin{array}{l}\text { Pillar } \\
\text { Aspect } \\
\text { Ratio } \\
\text { (height/ } \\
\text { width) }\end{array}$ & Material & $\begin{array}{l}\text { In-plane } \\
\text { lattice } \\
\text { parameter, } \\
a(\AA)\end{array}$ & $\begin{array}{l}\text { Out-of-plane } \\
\text { lattice } \\
\text { parameter, } c \\
(\AA)\end{array}$ & $\begin{array}{l}\text { In- } \\
\text { plane } \\
\text { strain }\end{array}$ & $\begin{array}{l}\text { Out-of- } \\
\text { plane } \\
\text { strain }\end{array}$ \\
\hline \multirow[t]{2}{*}{1} & \multirow[t]{2}{*}{12.8} & \multirow[t]{2}{*}{0.7} & $\mathrm{CoFe}_{2} \mathrm{O}_{4}$ & $8.34 \pm 0.01$ & $8.383 \pm 0.004$ & -0.005 & 0.000 \\
\hline & & & $\mathrm{BiFeO}_{3}$ & $3.918 \pm 0.003$ & $4.045 \pm 0.001$ & -0.011 & 0.020 \\
\hline \multirow[t]{2}{*}{2} & \multirow[t]{2}{*}{43.4} & \multirow[t]{2}{*}{1.2} & $\mathrm{CoFe}_{2} \mathrm{O}_{4}$ & $8.37 \pm 0.01$ & $8.386 \pm 0.002$ & -0.001 & 0.001 \\
\hline & & & $\mathrm{BiFeO}_{3}$ & $3.902 \pm 0.003$ & $4.063 \pm 0.001$ & -0.016 & 0.025 \\
\hline \multirow[t]{2}{*}{3} & \multirow[t]{2}{*}{150} & \multirow[t]{2}{*}{2.7} & $\mathrm{CoFe}_{2} \mathrm{O}_{4}$ & $8.37 \pm 0.01$ & $8.374 \pm 0.004$ & -0.001 & -0.001 \\
\hline & & & $\mathrm{BiFeO}_{3}$ & $3.926 \pm 0.003$ & $4.034 \pm 0.001$ & -0.010 & 0.017 \\
\hline
\end{tabular}

Table 6-1. Lattice parameters, strain conditions and anisotropy values for composite films in the study.
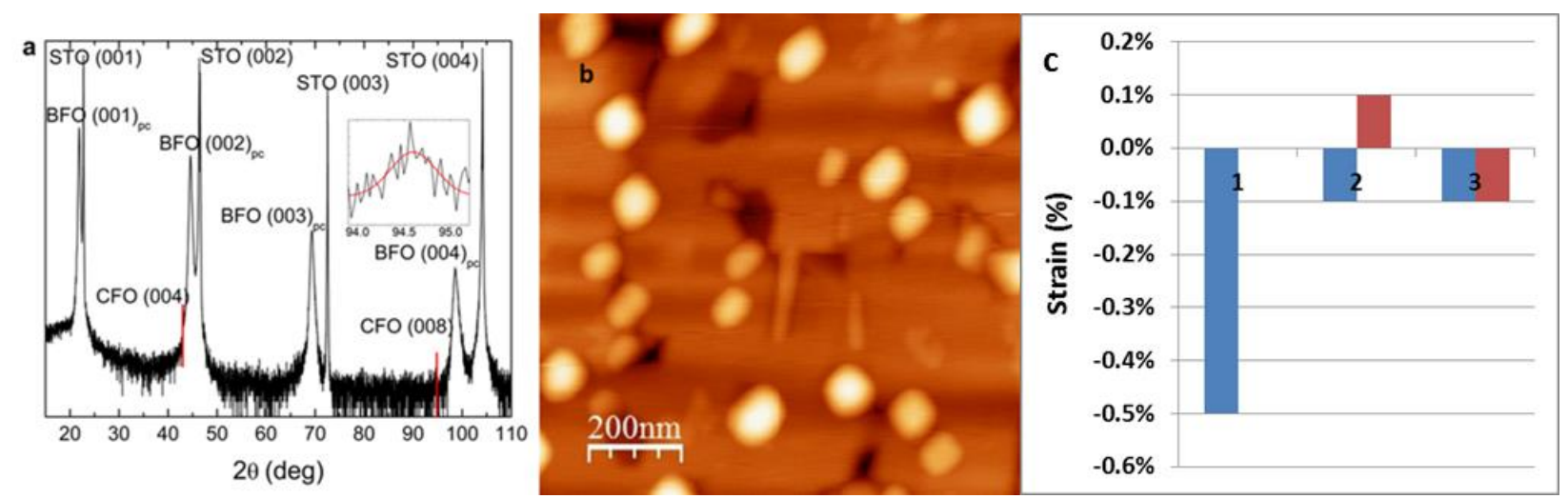

Figure 6-1. a) XRD measurement for Sample 2 (43 nm BFO thickness), with the CFO (008) peak inset and Gaussian curve fit to peak; b) AFM topography of Sample 2; c) Graph depicting in-plane (blue) and out-ofplane (red) strain conditions for samples examined in this section. 
For all three samples, XRD results indicated that the CFO pillars are relaxed along the out-of-plane axis. For the thinnest sample, the in-plane CFO lattice parameter is $8.34 \AA$, which is a compressive strain of $0.5 \%$ relative to the bulk lattice parameter. In the $43 \mathrm{~nm}$ sample and $150 \mathrm{~nm}$ sample, a slight in-plane compressive strain of $0.1 \%$ was found. These results disagree somewhat with previous results, which have often found out-of-plane compressive strain in the CFO pillars due to the lattice mismatch between CFO and the BFO matrix. ${ }^{78,79}$ Zhan et al. had previously shown that in-plane strain in BFO-NFO composites with thicknesses of greater than $100 \mathrm{~nm}$ was fully relaxed through the formation of misfit dislocations at the NFO-BFO lateral interface. ${ }^{72}$

\section{Model of Strain Relaxation in Pillars}

Some works have reported perpendicular magnetic anisotropy in CFO-BFO composites due to out-of-plane compressive strain in the CFO films. ${ }^{78,79,80}$ This strain is believed to arise from the vertical interface between the $\mathrm{CFO}$ pillar and the $\mathrm{BFO}$ matrix, which, if coherent or semicoherent, yield a compressive strain. However, others have demonstrated through transmission electron microscopy (TEM) analysis that the interface between similar $\mathrm{NiFe}_{2} \mathrm{O}_{4}(\mathrm{NFO})$ inverse spinel pillars and the BFO matrix is fully relaxed along the in-plane and out-of-plane

directions. $^{72}$ Additionally, most works have examined composite films with thicknesses much greater than $100 \mathrm{~nm}$, which would induce perpendicular magnetic shape anisotropy in the pillars. Thus, the origin of the anisotropy found in some composite films is not fully understood.

The presence of CFO in-plane strain for ultrathin BFO-CFO nanocomposites is an unexpected result that conflicts with previous studies in the literature. Most works have found that residual perpendicular compressive strain is present in the CFO pillars, which has been attributed to the lattice mismatch between BFO and CFO. To our knowledge, there have been no studies showing in-plane compressive strain in the CFO pillars. However, the films grown in this 
work are grown by a unique method when compared to those that are typically presented in the literature: the growth rate is slower and the film thickness is several times smaller than most other composites. The analysis of Zhan et al. ${ }^{72}$ showed that the equilibrium strain state along the out-of-plane axis is for NFO pillars to be fully relaxed through the formation of misfit dislocations at the BFO-NFO interface. ${ }^{72}$ Given that NFO and CFO share the same inverse spinel structures and have comparable lattice parameters $(8.38 \AA \text { for } \mathrm{CFO} \text { and } 8.34 \AA \text { for NFO })^{39}$, it seems reasonable to conclude that when these films grow more slowly any residual out-of-plane compressive strain is relaxed. This can be explained by considering that during film growth the addition of a repeating dislocation every $N$ unit cells with Burgers vector along the [001] direction is sufficient to relax away perpendicular strain. This was observed in the work of Zhan et al for the NFO-BFO vertical interface, with the value of $N$ equal to 20, which is approximately equal to the inverse of the lattice misfit between NFO and BFO. ${ }^{72}$ However, in-plane compressive strain in the pillars is more unusual. To explain this result, we consider the dislocation mechanics to relax strain within the pillars. Extra half planes form within the BFO matrix along the $\langle 110\rangle$ directions as an interfacial strain reduction mechanism. ${ }^{72}$ In the same way that uniform thin films have an expected critical thickness for the formation of misfit dislocations, it appears that there is thickness dependence for the density of misfit dislocations between the matrix and pillar. The thinnest film examined in this study has a BFO matrix that is $13 \mathrm{~nm}$ thick with in-plane pillar strain of $0.5 \%$. Given that the bulk lattice mismatch between $\mathrm{BFO}$ and $\mathrm{CFO}$ is $5.7 \%$, the pillars are mostly relaxed at this thickness. Further studies are needed on thinner films to measure the dislocation density as a function of thickness. Additionally, the mechanism for misfit dislocation formation in this system is not entirely clear. Detailed analysis of the Burger's vector and slip system via transmission electron microscopy could prove fruitful, 
but is beyond the scope of this work. A schematic representation of the hypothesized relaxation process in both the in-plane and out-of-plane directions is shown in Figure 6-2.

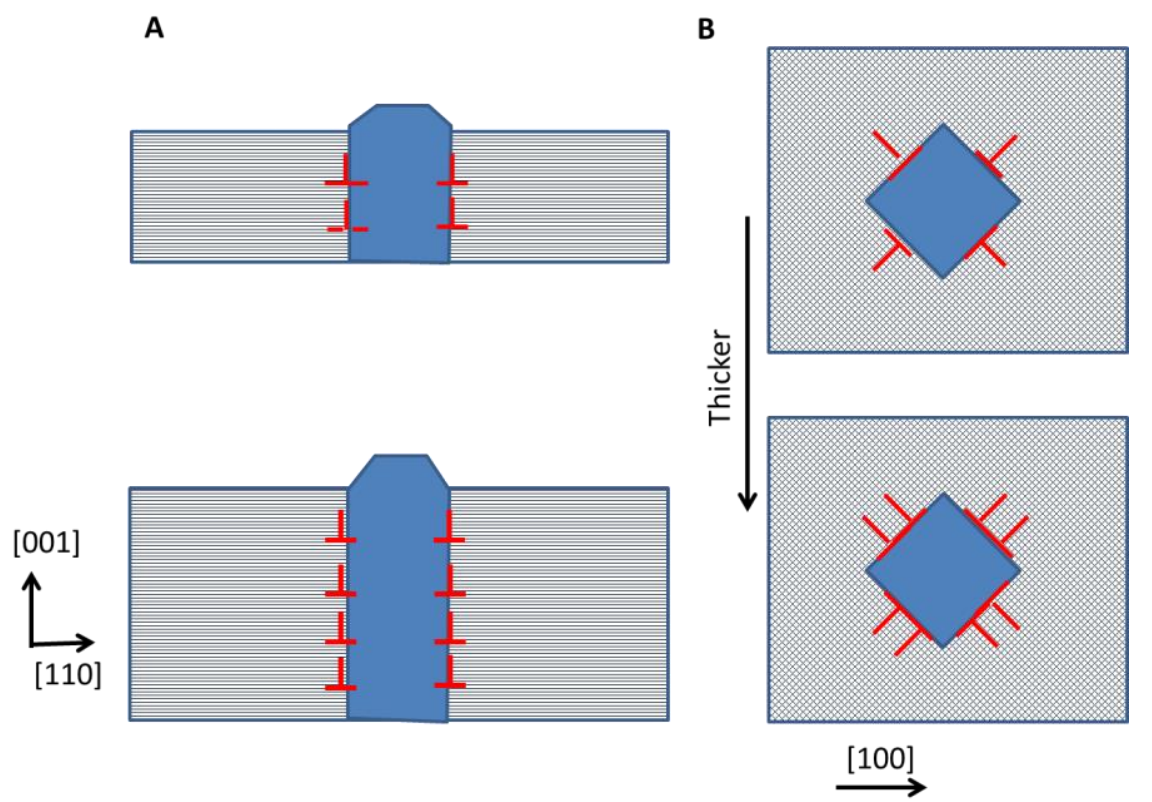

Figure 6-2. Models of strain relaxation (A) along the perpendicular axis and (B) along the in-plane $\langle 110\rangle$ axes as the thickness of the nanocomposite increases.

\section{Transmission Electron Microscopy Analysis of Nanocomposite}

To further examine the structural properties of the nanocomposite, an additional sample was grown on $\mathrm{Nb}$-doped STO with a thickness of approximately $20 \mathrm{~nm}$. A low density of CFO pillars was chosen so as to make comparisons with a patterned sample that will be discussed in the next chapter more straightforward. The sample was characterized using x-ray diffraction, MFM and VSM to confirm that it exhibited similar properties to those of the previous nanocomposites. Out-of-plane x-ray diffraction showed Kiessig fringes around the BFO diffraction peak, indicating excellent film quality. The presence of Kiessig fringes in a nanocomposite has thus far not been observed in the literature, which can be attributed to the fact that films presented in the literature are generally much thicker and have a greater area density of CFO pillars. Because of the thin film and low density of CFO pillars, it was not possible to resolve the $\mathrm{CFO}$ diffraction peaks above the background noise and nearby BFO peak in the measurement. 
The out-of-plane pseudocubic BFO lattice parameter was found to be $4.076 \AA$ A. A reciprocal space map about the STO (103) peak was performed and confirmed that the BFO film is coherently strained to the substrate. VSM measurements showed significant in-plane anisotropy, which is in agreement with the results that will be presented in the following section. The results of these measurements are summarized in Figure 6-3.
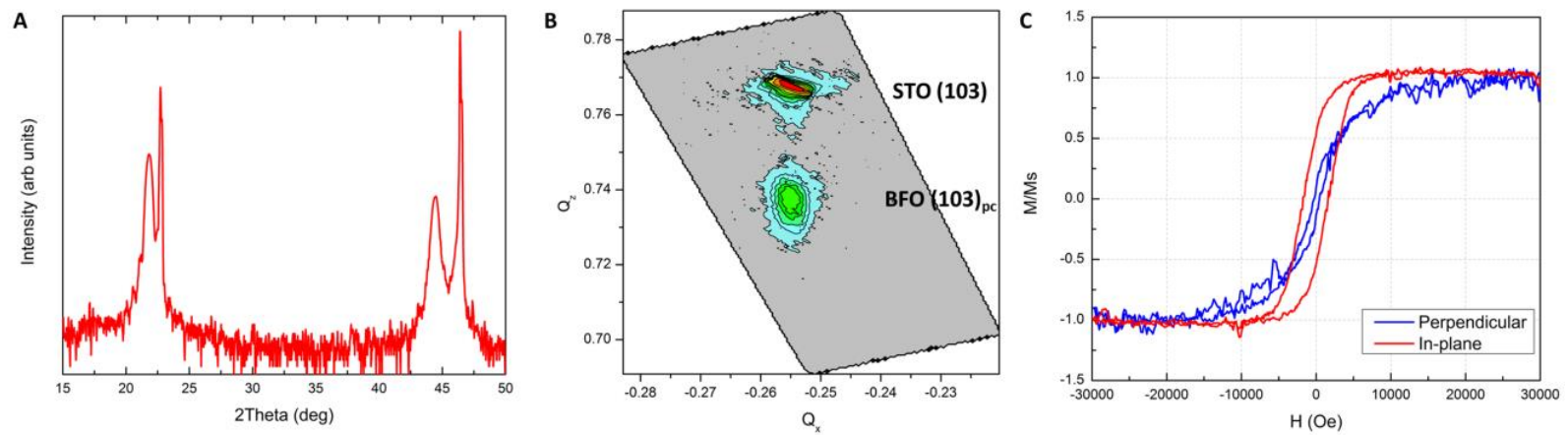

Figure 6-3. (A) Out-of-plane x-ray diffraction measurement for $20 \mathrm{~nm}$ thick BiFeO3-CoFe2O4 nanocomposite; (B) Reciprocal space map about SrTiO3 (103) peak; (C) In-plane and out-of-plane magnetic hysteresis loops of sample.

A cross-sectional lamella of the sample was then prepared using the focused ion beam liftout technique. The lamella was prepared so that the cross-sectional zone axis would be along the $\langle 110\rangle$ direction, making the $\{110\}$ facetted CFO nanopillars have uniform width along the cross-section. The sample was then characterized using high-angle annular dark field scanning transmission electron microscopy (HAADF-STEM), energy dispersive x-ray spectroscopy (EDS) and high-resolution transmission electron microscopy (HRTEM). HRTEM was used to examine the various interfaces in the nanocomposite system, while EDS and HAADF-STEM were used to characterize the chemical properties of the composite.

A wide-view HAADF-STEM image is shown in Figure 6-4. Two CFO nanopillars are visible in the image as gray pentagon-shaped structures embedded in the bright BFO matrix. Because HAADF-STEM is sensitive to the atomic number, $Z$, of the elements in the material, BFO produces the brightest contrast in the image because it has atomic number $83 . \mathrm{Sr}, \mathrm{Ti}, \mathrm{Co}$ and $\mathrm{Fe}$ on the other hand have atomic numbers 
of 38, 22, 27 and 26 respectively. Thus, the $\mathrm{Nb}$ :STO substrate will have somewhat brighter contrast than the CFO pillars. The dark region above the surface of the film is amorphous carbon that was deposited during the sample preparation process as a protective barrier.

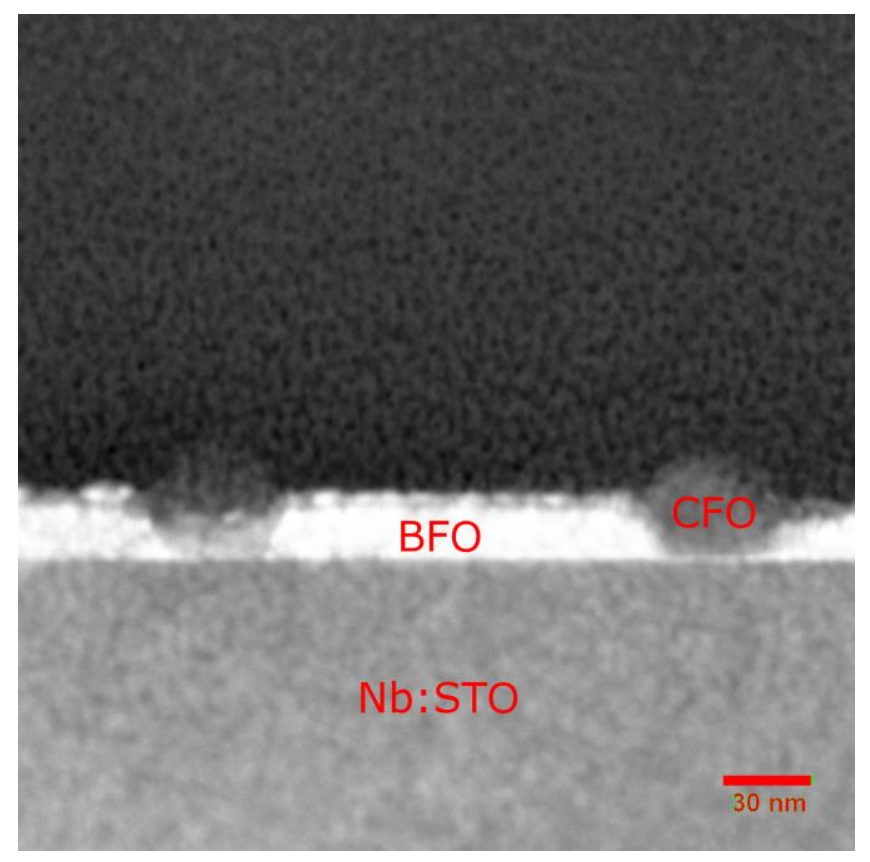

Figure 6-4. HAADF-STEM image of two nanopillars viewed along the $\langle 110\rangle$ zone axis.

The unusual shape of the pillars compared to the vertical structure that one would expect to see in a pillar is due to the film's reduced thickness compared to what is commonly grown in the literature. For example, in Zheng et al. ${ }^{71}$, the authors grew CFO-BFO nanocomposites that were several hundred nm in thickness. In this work, they observed that the pillars did not form $\{110\}$ in-plane facets during the growth process until the pillars were more than $50 \mathrm{~nm}$ in height. ${ }^{71}$ The pillars initially formed as small islands and then expanded outwards as the thickness increased, before maintaining a uniform diameter at thickness of greater than $50 \mathrm{~nm}$. Thus, because this sample is only $25 \mathrm{~nm}$ thick, one would expect the pillars to have less defined diameters as they expand outward and reach an equilibrium diameter.

To confirm the hypothesis of the chemical makeup of the nanocomposite, an EDS map was obtained with $2 \mathrm{~nm}$ spatial resolution around a nanopillar. The Ti $K_{\alpha}$, Co $K_{\alpha}$, and Bi $L_{\alpha}$ peaks were then isolated in 
the spectra and images were obtained showing the intensity of each element in the sample at each point. The full EDS map is shown in Figure 6-5, with the individual element maps shown in Figure 6-5(B-C) and an overlay of the images shown in Figure 6-5(A). It is important to note that the Co $K_{\alpha}$ peak lies very near the $\mathrm{Fe} K_{\beta}$ peak on the energy spectrum. Thus, there is non-zero Co intensity observed in the BFO matrix, which is an artifact of the measurement and is not an indication of Co presence in the BFO lattice. This makes quantitative analysis of the Co concentration in the matrix impossible. The Bi map (Figure 6-5 (C)) shows that there is some BFO that overlaps with the CFO nanopillar. This is the result of the sample preparation process and the geometry of the pillar, rather than a sign of Bi residing within the CFO crystal lattice. The thickness of the lamella is greater than the diameter of the nanopillar at the base of the pillar, meaning that some BFO is present in front of and behind the nanopillar. This explanation will be confirmed later when discussing the HRTEM images. The Co map (Figure 6-5 (B)) shows a reduced intensity above the matrix surface, which is the result of the reduced pillar thickness in that region due to the $\{111\}$ faceting structure. The Ti map (Figure 6-5(D)), show that the substrate is uniform and homogeneous across the scanning area, suggesting that there has not been significant chemical change in the substrate after growth. This is further confirmed by the absence of a detected signal in the $\mathrm{Co}$ and $\mathrm{Bi}$ maps in the substrate region. 


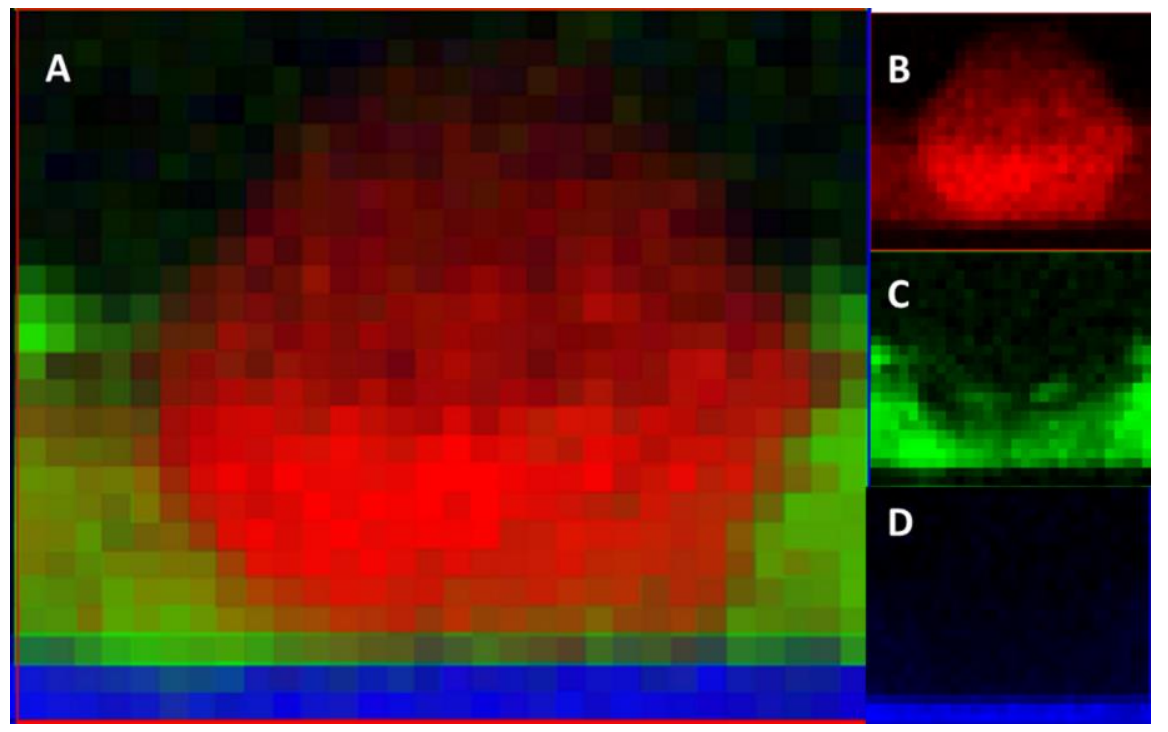

Figure 6-5. EDS map of $\mathrm{CoFe}_{2} \mathrm{O}_{4}$ nanopillar embedded in $\mathrm{BiFeO}_{3}$ matrix. A) Overlay of all three elements mapped; B) Intensity of Co $K_{\alpha}$ peak; C) Intensity of Bi $L_{\alpha}$ peak; D) Intensity of Ti $K_{\alpha}$ peak.

To analyze the interfacial structure and strain in the nanopillars, HRTEM was employed. While several pillars were examined, this analysis will focus on the pillar presented above for EDS analysis. Other pillars exhibited similar interfacial and crystallographic features. Figure 6-6 shows a HRTEM image of the pillar under examination, with a fast Fourier transform (FFT) image of the region shown in the inset. The FFT image is analogous to a selected-area electron diffraction pattern acquired directly via TEM in many other works. ${ }^{132}$ The FFT image along the $\langle 110\rangle$ zone axis shows the same diffraction pattern as other nanocomposite samples in the literature along this zone axis. ${ }^{71}$ The CFO peaks are clearly distinct from the perovskite peaks that result from the BFO matrix and $\mathrm{Nb}$ :STO substrate, indicating that the strain in the pillar is mostly relaxed. At this resolution, it is not possible to precisely state whether there is any residual strain in the pillar either in-plane or out-of-plane. Analysis of the FFT pattern did indicate that the strain was in agreement with a fully relaxed pillar, but could not rule out some degree of strain along either direction. Separation of the BFO and Nb:STO lattice parameters was not possible in this particular image. Thus, confirming the hypothesis discussed in Section 1.2 of this chapter will require more detailed analysis using other techniques. 


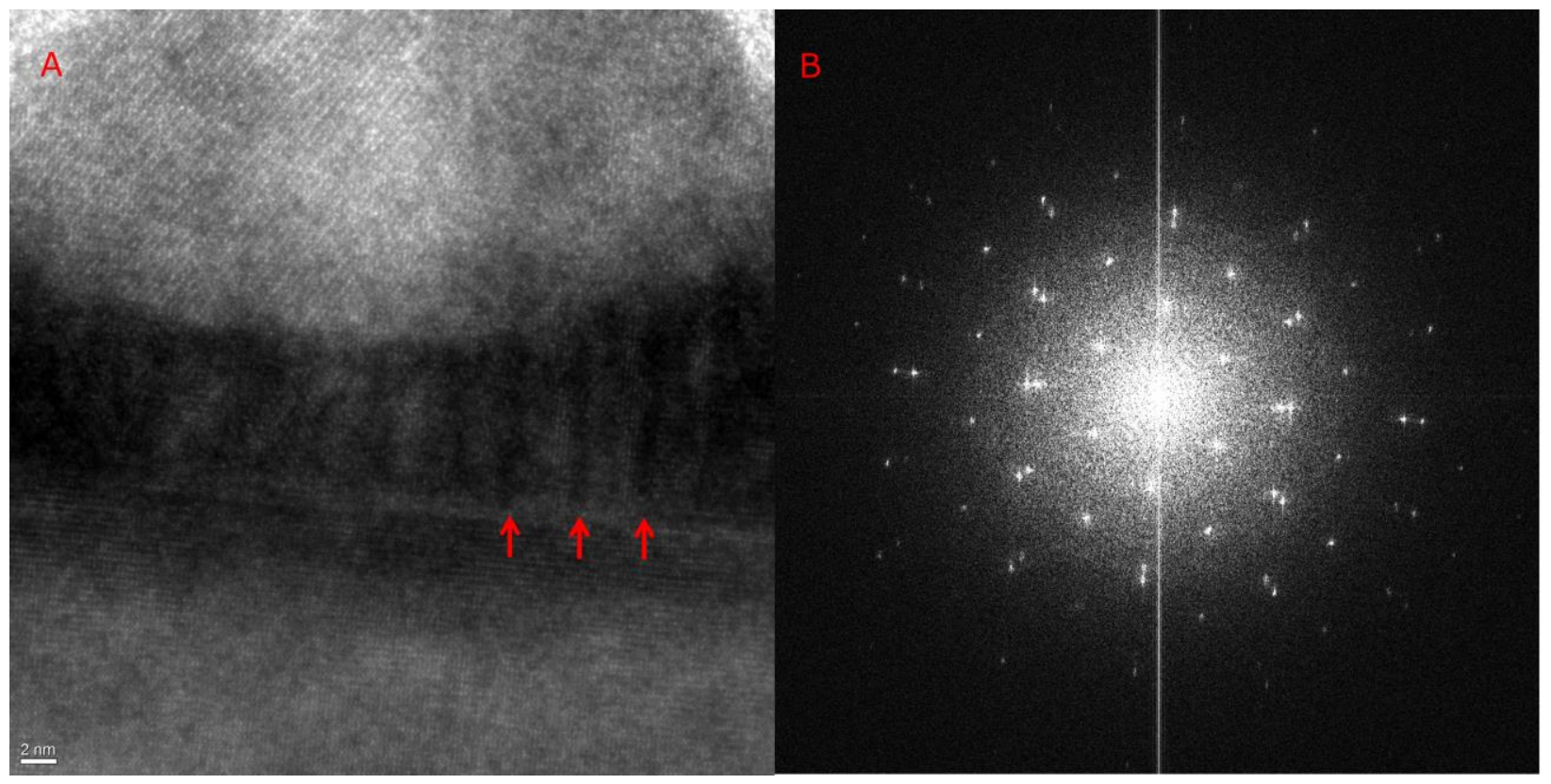

Figure 6-6. A) HRTEM image of pillar measured via EDS in Figure 6-5. Arrows indicate examples of dark Moire fringes in pillar. B) Fast Fourier transform of entire field in A.

Figure 6-6(A) also shows the presence of Moire fringes at in front of the CFO pillar, which are visible as periodically spaced light and dark stripes. Arrows in Figure 6-6(A) indicate examples of the dark bands of the Moire fringes. Moire fringes occur as an interference effect when a TEM image is acquired with the beam passing through two incoherent crystal lattices. ${ }^{132}$ The pattern of Moire fringes in this pillar shows the same feature shape as the $\mathrm{Bi} L_{\alpha}$ edge map in Figure 6-5(C). This confirms the argument put forth above regarding the presence of residual BFO either in front of or behind the CFO pillar along the path of the beam.

To analyze the different phases present in the material in more detail, selected area FFT analysis was performed on a separate image showing both the matrix and the pillar. These results are shown below in Figure 6-7. Selected area FFT images of the Nb:STO substrate (B), the BFO matrix (C) and the CFO pillar above the surface of the matrix (D) were all acquired to independently index the peaks in reciprocal space. In the substrate, only a cubic diffraction pattern is evident with the (110) and (001) peaks noted. In the BFO matrix, the same pseudocubic peaks are observed, but additional peaks at shorter distances from 
the origin in reciprocal space are also observed. These peaks cannot be indexed in the pseudocubic scheme used throughout this work, but occur as a result of the rhombohedral distortion of the perovskite crystal in BFO. This distortion produces a crystal structure that can be indexed by assuming a hexagonal unit cell along the $[111]_{\mathrm{pc}}$ direction. ${ }^{171}$ The hexagonal index is in agreement with the pattern shown in Figure 6-7(C). A green diamond is shown as a guide to the eye, connecting two of the non-indexed peaks to the $(0 \overline{01})$ peak. The diffraction pattern in Figure 6-7(D) for the CFO pillar shows a similar hexagonal pattern, which is expected for the [110] zone axis in CFO since it exhibits a face-centered cubic crystal structure. ${ }^{132}$ A strong (004) diffraction peak is observed, along with a (220) peak, which are the result of the unit cell doubling that occurs in the spinel oxides. A weak (002) peak is also observed, in spite of the fact that it is a forbidden peak based in the $F d \overline{3 m}$ space group. The presence of this weak peak can most likely be attributed to point defects such as anti-site defects in the CFO pillar, where Co resides on the spinel A-site instead of $\mathrm{Fe}$, or oxygen vacancies that result during growth. A green diamond is again shown as a guide to the eye, showing the hexagonal pattern in the FFT image.

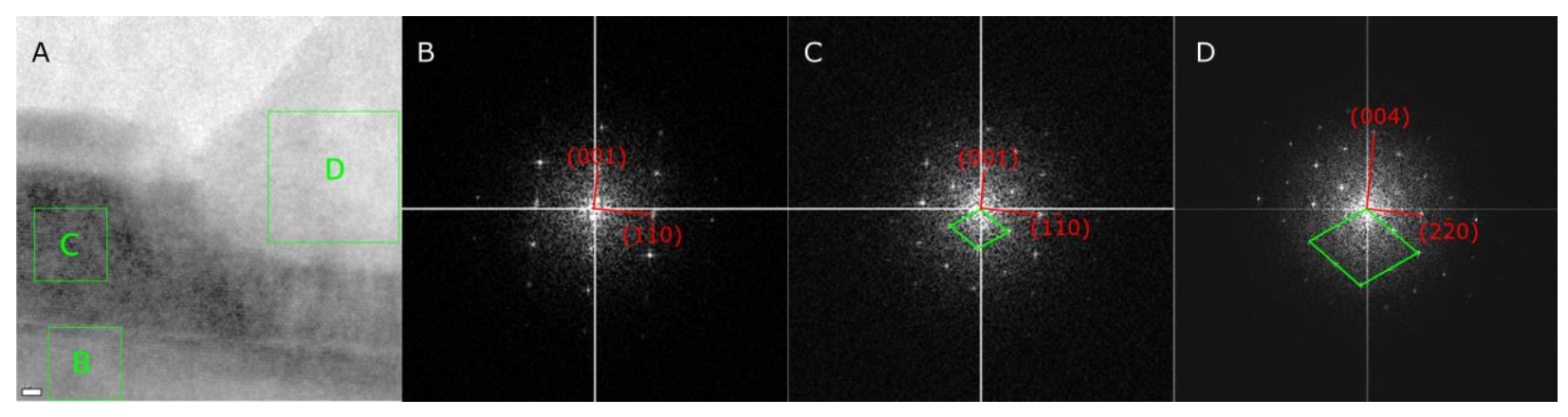

Figure 6-7. HRTEM FFT analysis of substrate pillar and matrix of image in (A). Annotations in image show selected regions for FFT images in following images. B) Nb:STO substrate; C) BFO matrix; D) CFO pillar. Cubic or pseudocubic indices are noted in all three images with low index diffraction peaks. Green diamonds showing hexagonal pattern of peaks are shown as a guide to the eye in (C) and (D).

\section{Magnetic Properties of Nanocomposites}

\section{Thickness Dependent Magnetic Anisotropy}

To determine if any magnetic anisotropy was present in the films, VSM measurements were made along both the in-plane [100]-direction and the out-of-plane [001]-direction for the samples. The results 
of these measurements are shown in Figure 6-8(a-c). For the thinnest sample, in-plane anisotropy is clearly present, with the in-plane coercivity 1.3 kOe greater than the perependicular coercivity. In Sample 2 , there is slight in-plane anisotropy, as the coercivity is $1.6 \mathrm{kOe}$ in-plane and $1.3 \mathrm{kOe}$ out-of-plane. For the thickest film, the hysteresis loops are nearly identical, with no difference in coercivity or remanence between scans.
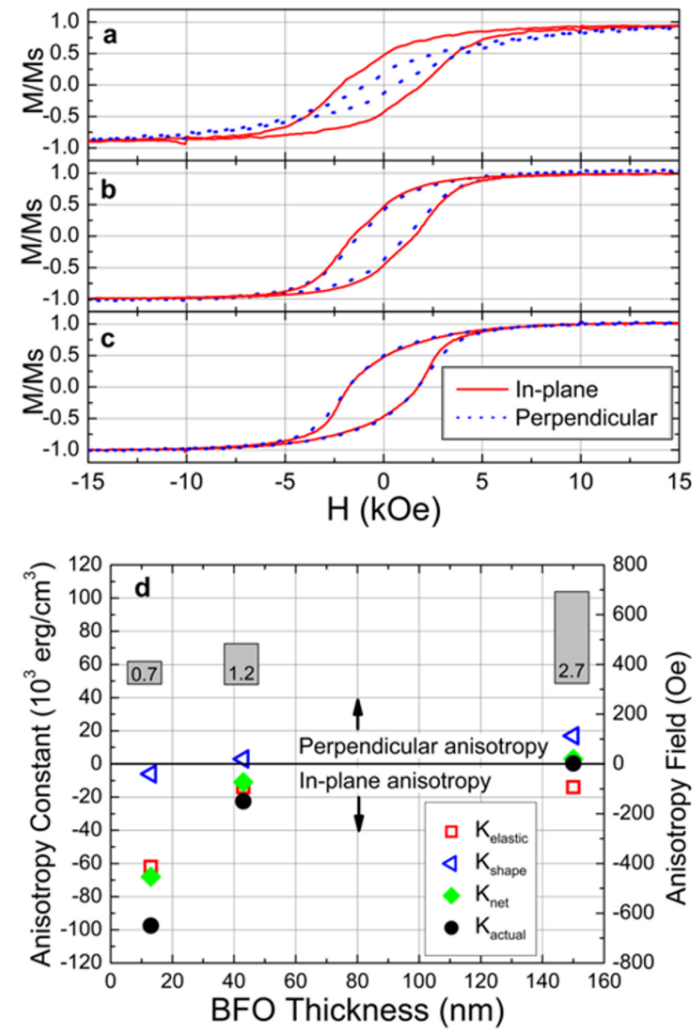

Figure 6-8. a-c) Magnetic hysteresis loops for CFO-BFO composite film along in-plane [100] and out-of-plane [001] directions. a) Sample 1, with BFO thickness of $13 \mathrm{~nm}$; b) Sample 2, with BFO thickness of $43 \mathrm{~nm}$; c) Sample 3, with BFO thickness of $150 \mathrm{~nm}$. d) Predicted magnetoelastic ( $K_{\text {elastic }}$, square), shape $\left(K_{\text {shape }}\right.$, triangle) and net ( $K_{\text {net }}$, diamond) uniaxial anisotropy constants (left axis) and fields (right axis) for each film based on strain and pillar aspect ratios. Measured anisotropy fields $\left(K_{\text {actual }}\right.$, circle $)$ are also shown. Rectangles are drawn with the pillar aspect ratio of each sample.

In order to confirm these results and ensure that the pillars were the source of the magnetic signal, magnetic force microscopy (MFM) was performed on Sample 2 from Table 6-1. Results of this measurement are shown in Figure 6-9. The pillar was demagnetized in a perpendicular magnetic field and 
then measured with no applied field via MFM. The results showed that only the CFO pillars were ferromagnetic at room temperature, and that the majority of the pillars had in-plane magnetization after being demagnetized in a perpendicular field. In-plane magnetization in a pillar was inferred from a change from red to blue contrast across the width of the pillar, which can be seen in the red circle highlighting a pillar in Figure 6-9(B). The change in contrast results from the curling of the in-plane magnetic dipole moment over the pillar, which produces a positive out-of-plane field on one side of the pillar and a negative out-of-plane field on the other side. Perpendicular magnetization was highlighted in the red box, where a pillar had uniform color contrast across the entire diameter.

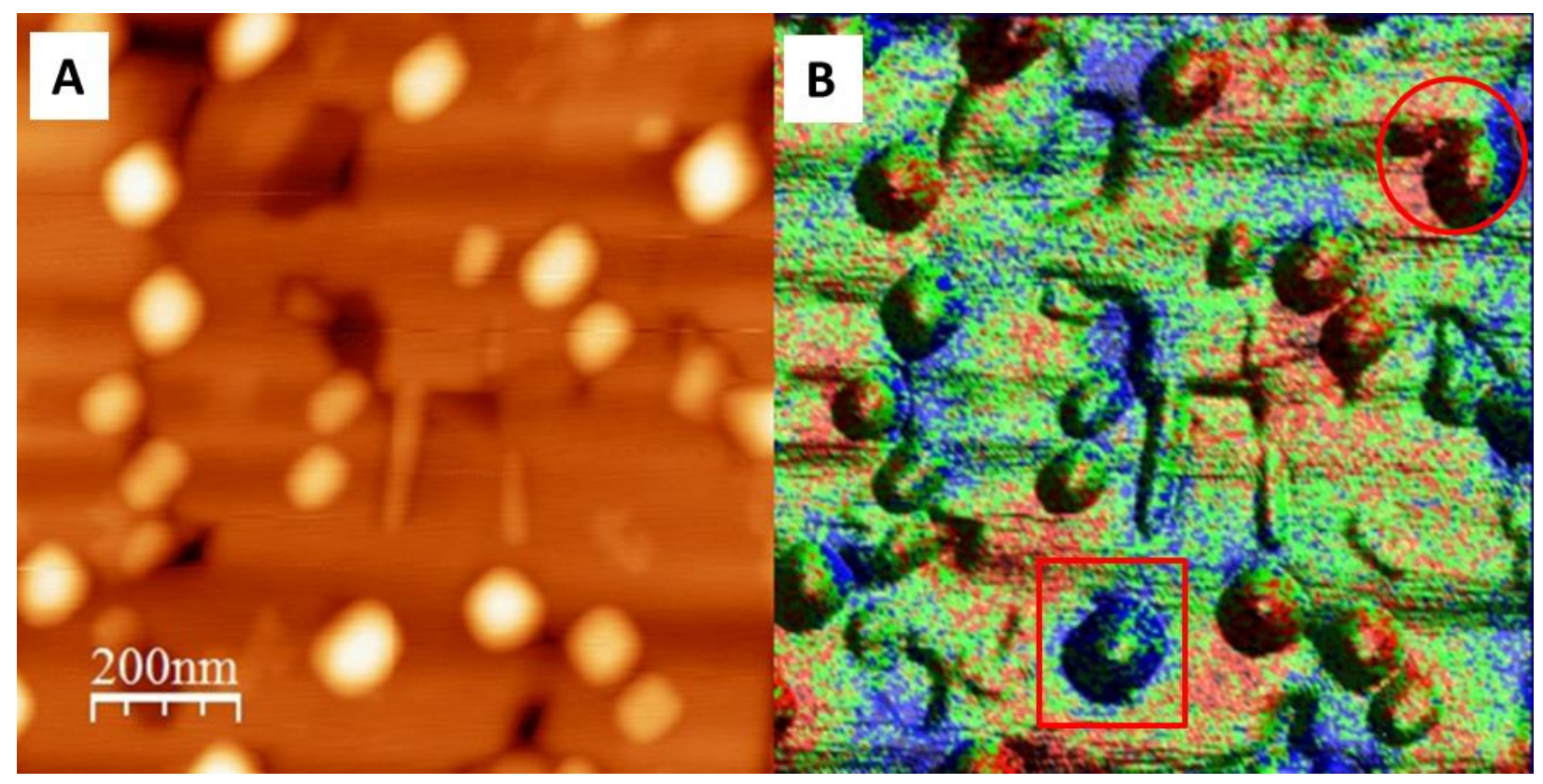

Figure 6-9. Magnetic force microscopy image of nanocomposite. A) Topography; B) Phase overlaid as color on three-dimensional rendering of topography. Red and blue represent positive and negative out-of-plane fields (arbitrary sign), while green is neutral. The circle highlights a pillar with in-plane magnetization, while the box highlights a pillar with perpendicular moment.

\section{Discussion of Properties}

We consider the combined effects of shape and magnetoelastic anisotropy to explain the measured anisotropy. Using the published values for the CFO magnetostriction coefficient $\left(\lambda_{100}=\right.$ $\left.-590 \times 10^{-6}\right)^{41}$ and a Young's modulus of $140 \mathrm{GPa},{ }^{13}$ the expected magnetoelastic anisotropy 
values for each sample are calculated, using the formula $K_{m e}=3 / 2 \lambda_{100} \sigma_{100}$, where $\sigma_{100}$ is the inplane stress in the pillars. To determine the shape anisotropy, a model which treats the pillars as cylinders was chosen since typical rectangular models assume magnetization along the faces of the rectangular prism, rather than the edges as we have for the $\left\langle 110>\right.$-faceted pillars. ${ }^{172}$ The shape anisotropy values for each aspect ratio are calculated assuming a saturation magnetization of $350 \mathrm{emu} / \mathrm{cc}$ for CFO. The predicted elastic, shape and net anisotropies are plotted in Figure 6-8(d), with $K_{\text {net }}=K_{\text {elastic }}+K_{\text {shape }}$. The anisotropy field, $H_{k}=2 K / M_{s}$, for these samples is also shown. ${ }^{131}$ The measured anisotropy field was determined by taking half of the difference in the coercivities of the in-plane and out-of-plane hysteresis loops and is also shown. Both shape and elastic anisotropies contribute to the large in-plane anisotropy found in Sample 1. In-plane elastic anisotropy is partially canceled by a slight out-of-plane shape anisotropy in Sample 2. The larger pillar aspect ratio in Sample 3 increases the shape anisotropy and yields a very small predicted out-of-plane anisotropy field, which is within the margin of error in the VSM measurement. The measured results show good agreement with the predicted values given the simplicity of the model for the shape anisotropy. Thus, the overall anisotropy in the system can be understood by considering the combined effects of shape and elastic anisotropies. The results also demonstrate that the contribution of the shape anisotropy to the net anisotropy of the pillar is substantially less than the contribution of elastic anisotropy. This is due to the large magnetostriction coefficient of CFO. In other spinel-perovskite nanocomposites, such as $\mathrm{NiFe}_{2} \mathrm{O}_{4}-\mathrm{BFO}$ composites, one would expect the shape anisotropy to play a larger role because the magnetostriction coefficient is substantially less in $\mathrm{NiFe}_{2} \mathrm{O}_{4}{ }^{39}$ 


\section{Ferroelectric Properties of Nanocomposites}

Prior to microscopy measurements, the sample discussed in Section C was characterized using piezoresponse force microscopy (PFM). Two separate approaches were used: Dual AC Resonance Tracking (DART) and vector PFM. In addition to the DART PFM measurements, switching-spectroscopy PFM (SS-PFM) was performed in the BFO matrix of the sample to measure a ferroelectric hysteresis loop. The results of the DART and SS-PFM measurements are shown in Figure 6-10, with the site of the SS-PFM measurement marked with an ' $\mathrm{X}$ ' in Figure 6-10(A). The measured domain structure shows that the film is primarily single domain out-of-plane, with a few small reversal domains with opposite polarity. These domains appear to originate at the interface between the CFO pillar and the BFO matrix, and may result from local point defects, such as oxygen vacancies, that are induced at the interface with the CFO pillar. ${ }^{173}$ Similar domain characteristics have been observed in PFM measurements of BFO-CFO nanocomposites performed by other groups. ${ }^{174}$ The amplitude image (Figure 6-10(B)) shows that the CFO pillars do not exhibit any ferroelectric response, with the blue regions of zero amplitude corresponding to the locations of the pillars in the topography image (Figure 6-10(A)). 


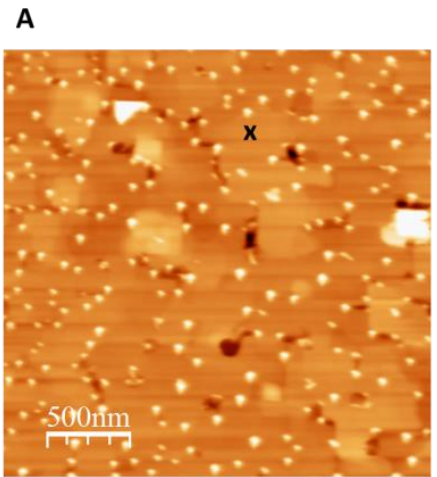

D
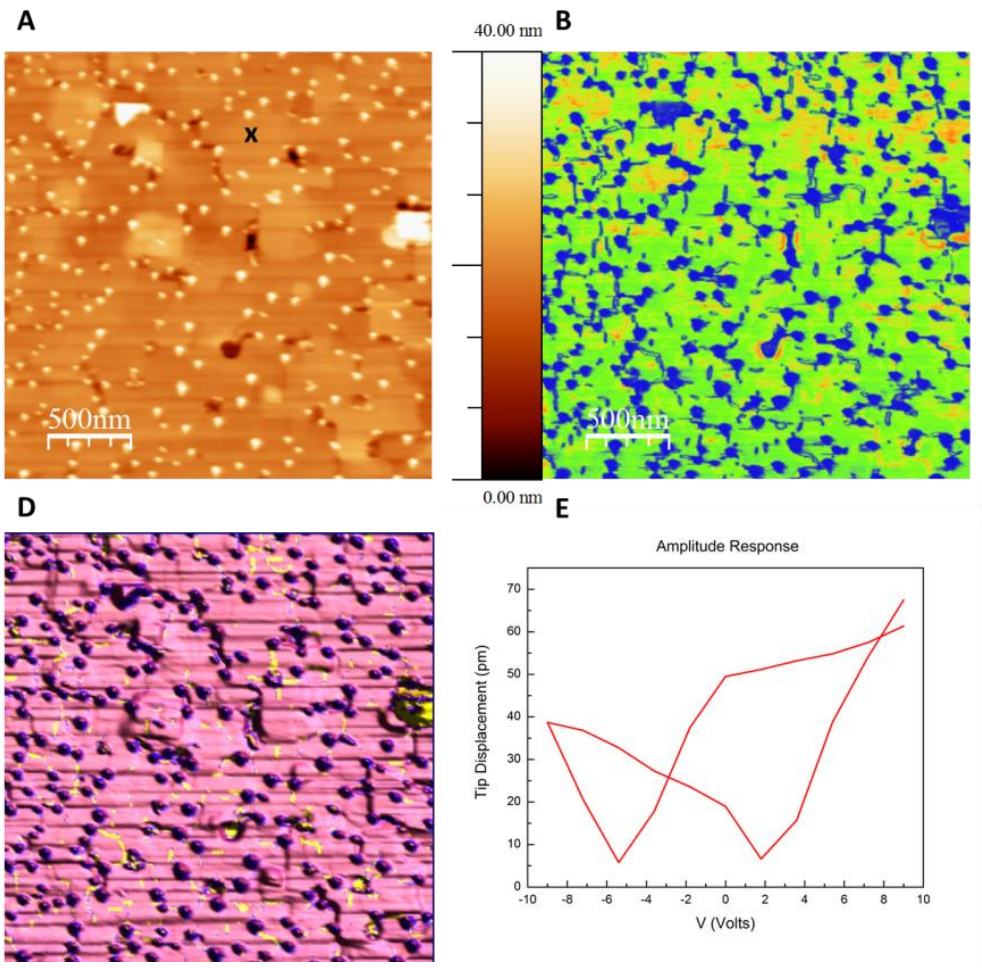

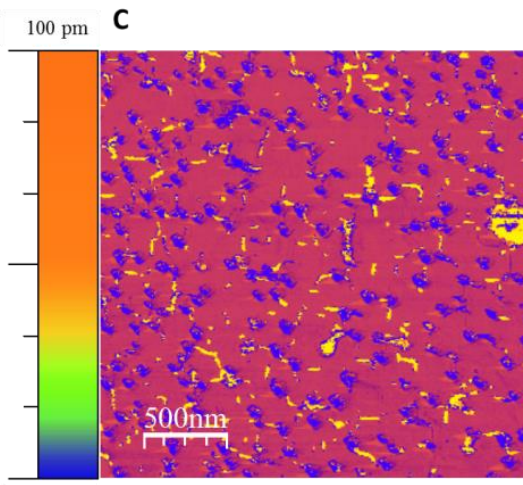

$0 \mathrm{pm} \quad \mathbf{F}$

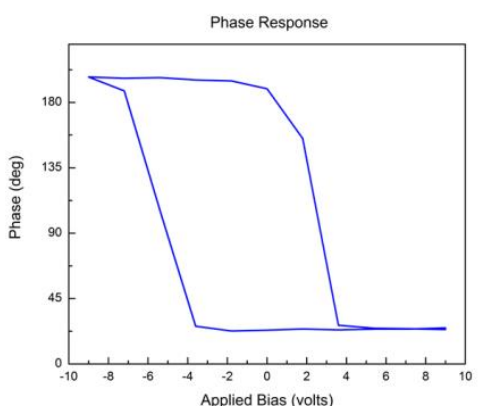

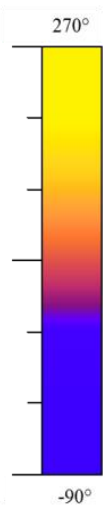
nanocomposite made using Dual AC Resonance Tracking (A) Surface topography; (B) PFM amplitude; (C) PFM out-of-plane phase; (D) 3D rendering of the surface topography with the out-of-plane phase overlaid. Switching-spectroscopy PFM (E) amplitude and (F) phase measurements made at the $X$ in (A).

SS-PFM measurements made in the matrix are shown in Figure 6-10(E-F). These hysteresis loops were acquired simultaneously using the conductive probe tip as an electrode. The amplitude response is shown in Figure 6-10(E) and exhibits the "butterfly" response that is expected for ferroelectric hysteresis. The phase response is shown in Figure 6-10(F) and shows complete $180^{\circ}$ switching of the out-of-plane polarization. The majority polarization of the matrix is approximately $20^{\circ}$, while the reversed polarization is approximately $200^{\circ}$. The hysteresis loop shows asymmetric switching, with the coercivity to reverse the polarization is between -5 and $-6 \mathrm{~V}$ applied bias, while the coercivity to return the polarization to the majority state is between 2 and $3 \mathrm{~V}$. This is likely the result of a significant depolarizing field due to the nature of ferroelectric domain formation when the PFM tip is used as an electrode. ${ }^{81}$ In order to reverse the polarization, a reversal domain must nucleate at the surface of the BFO matrix, where the applied electric field from the tip is at a maximum. This reversal domain must then travel through the thickness of 
the film and propagate outwards to create a sufficiently large out-of-plane domain to create a remanent state that can overcome the depolarizing field of the rest of the BFO matrix. ${ }^{175}$ However, when a smaller positive field is applied, the depolarizing field aids in the destruction of the reversal domain, which produces a smaller coercivity than for negative bias. This type of asymmetric hysteresis loop has been observed via SS-PFM in thicker BFO-CFO nanocomposites by other groups previously. ${ }^{174}$

For vector PFM measurements, both out-of-plane and in-plane ferroelectric domain structures in the BFO matrix were measured. Because the axis of polarization in BFO is along the pseudocubic <111> directions, there are expected to be 4 distinct in-plane polarization vectors even if the matrix is exhibits uniform out-of-plane domain structure. The results of the vector PFM measurement are shown in Figure 6-11. Figure 6-11(B-C) show the out-of-plane domain structure that was measured during the first pass of the scan, while Figure 6-11(E-F) show the in-plane domain structure which was measured during a second pass at a different drive frequency. The in-plane phase was overlaid as a color map onto a 3D rendering of the measured topography and is shown in Figure 6-11(D). 

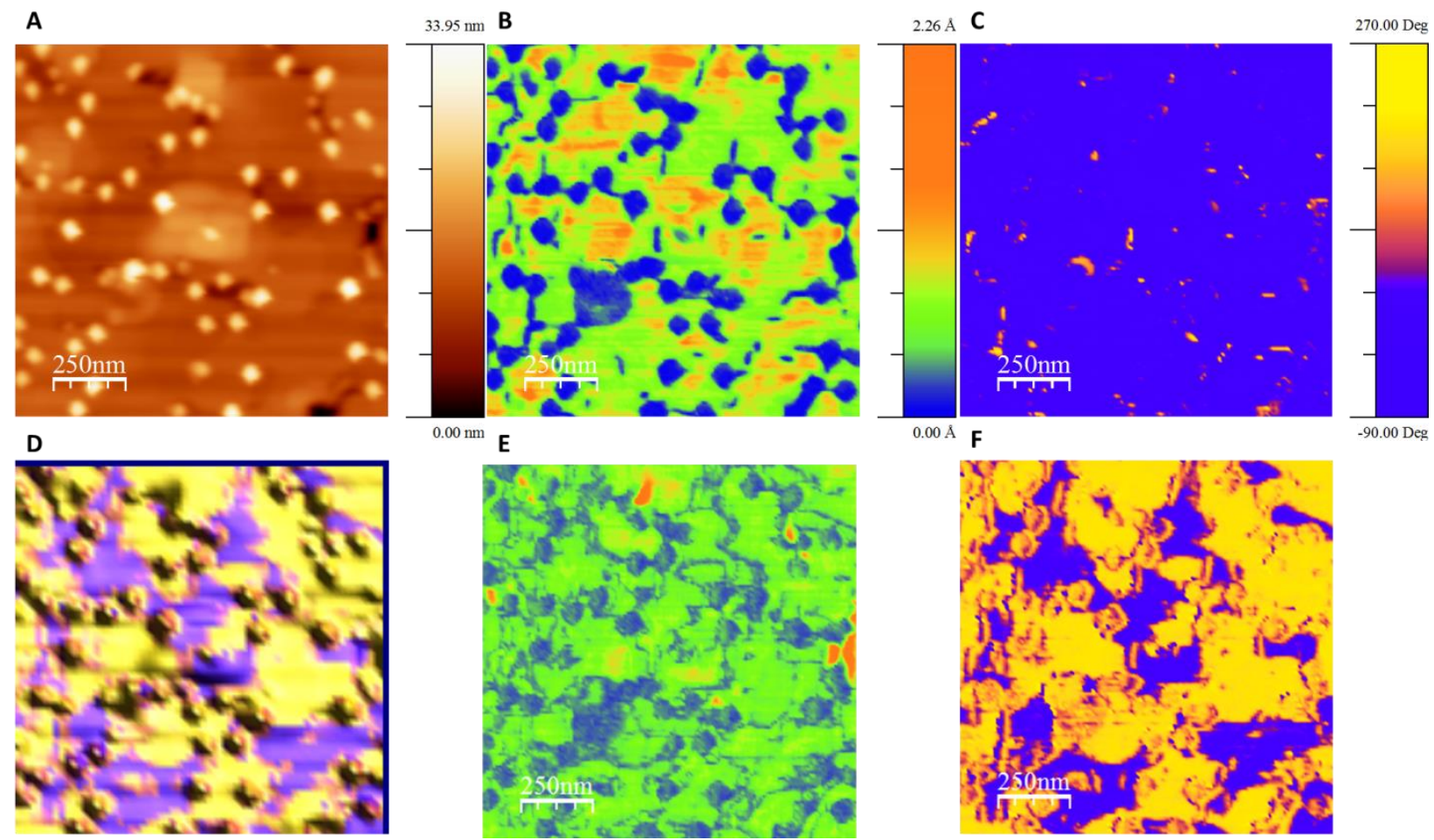

Figure 6-11. Vector piezoresponse force microscopy (PFM) measurements made on the same sample as in YY. (A) Sample topography; Out-of-plane (B) amplitude and (C) phase; (D) In-plane phase overlaid on 3D rendering of surface topography; In-plane (E) amplitude and (F) phase measurements with the same color maps as in the out-of-plane direction (arb. units for amplitude).

The out-of-plane domain structure matches the results obtained using the DART technique. The inplane domain structure is more complex, as the vector technique measures the polarization along the direction perpendicular to the scan axis. This means that only two possible polarization states can be observed, which will be out-of-phase $180^{\circ}$ from each other. To measure the polarization along the other axis, the sample must be physically rotated $90^{\circ}$ and a second scan must be performed over the same region. ${ }^{146}$ Without patterning of alignment marks to find the same region of interest for repeated scans this approach is impossible. However, it is possible to extract some information from a single component of the in-plane domain structure. Domains which were $180^{\circ}$ out-of-phase with each other were observed with roughly equal area coverage, indicating that there is symmetric polarization along the symmetric $<110>$ in-plane directions. Given that both in-plane directions are crystallographically equivalent for a cubic substrate, this is to be expected. There is also evidence that in-plane domain walls pin at the 
interface with the CFO pillars. In the in-plane amplitude image (Figure 6-11(E)) domain walls appear in blue because no ferroelectric response is present in these regions. Qualitative observations show that most domain walls start and end at a pillar site, indicating that it is likely that the interface between the matrix and pillar acts as a pinning site.

The observation of in-plane domain wall pinning in the nanocomposites is intriguing. A good deal of work in uniform BFO films has focused on the in-plane domain structure under a variety of strain conditions. Our group has found that for thin BFO films grown on $\mathrm{SrRuO}_{3}(\mathrm{SRO})$-buffered STO that the in-plane domain structure shows domains oriented along both directions with no evidence of pinning effects. ${ }^{176}$ In another work for uniform BFO films grown on SRO-buffered $\mathrm{DyScO}_{3}$, groups have shown that is possible to control the domain structure through the use of epitaxial strain from the substrate. ${ }^{177}$ This work showed that when a BFO film is grown via step-flow layer-by-layer growth on the $\mathrm{DyScO}_{3}$ substrate, the domain structure will produce uniform periodic stripe domains along the $[110]_{\mathrm{pc}}$ direction. This occurs due to the domain structure of the orthorhombic SRO buffer layer, which is single domain when grown via step-flow growth. The buffer layer seeds the growth to permit polarization only along two of the four possible in-plane <110> polarization directions. In another work, the vicinality of an STO substrate was used to control the in-plane domain structure of the BFO film. ${ }^{178}$ By choosing a variety of different substrate miscut angles for STO (001) substrates, the authors were able to produce different inplane domain configurations due to the broken symmetry induced by the high density of substrate step edges. For the highest miscut angle of $3^{\circ}$, they demonstrated a single domain polarization in-plane. In this work, however, an entirely different mechanism to control the domain structure has been used.

No previous work has been performed examining the in-plane domain structure of the BFO matrix in CFO-BFO nanocomposite films. The sample examined here is very unique compared to other films studied in the literature. The density of CFO pillars is significantly lower than in other works and the film thickness is roughly one order of magnitude less, producing a coherently strained BFO matrix. Given that in-plane domain walls appear to pin at pillar sites, a lower density of CFO pillars would produce larger 
BFO domains to examine. The cause of the domain wall pinning is most likely due to the structural defects induced at the interface between the pillar and matrix. This work has shown that the BFO-CFO interface is incoherent, which necessitates a high density of misfit dislocations surrounding the vertical interface. Other groups have also shown that oxygen vacancies occur with a higher density at the vertical interface. ${ }^{173}$ Each of these could promote domain wall pinning at the interface. Further exploration of these findings is needed. Through the use of directed self-assembly of the CFO nanopillars, it might be possible to control the in-plane domain structure in a systematic way. ${ }^{100}$ Initial results in this study will be explored in the following chapter.

\section{Summary}

A series of $\mathrm{CoFe}_{2} \mathrm{O}_{4}(\mathrm{CFO})-\mathrm{BiFeO}_{3}(\mathrm{BFO})$ epitaxial nanocomposites grown by pulsed electron deposition (PED) has been presented in this chapter and their microstructural and multiferroic properties have been examined. Unexpected in-plane compressive strain was observed in the CFO pillars for ultrathin samples. This result was attributed to the lattice mismatch between CFO and the STO substrate and BFO matrix, which both promote in-plane compressive strain. In addition, the absence of out-ofplane compressive strain in the $\mathrm{CFO}$ pillars was surprising. This difference from the literature was explained by considering the different growth kinetics between the PED technique employed here and the more commonly used pulsed laser deposition (PLD) technique. By reducing the growth rate, complete out-of-plane strain relaxation at the interface between the CFO pillar and BFO matrix is possible. The magnetocrystalline anisotropy of the CFO pillars was characterized via VSM and MFM and found to exhibit in-plane anisotropy for the thinnest samples. The in-plane anisotropy observed is expected for pillars with in-plane compressive strain, but has not previously been demonstrated in the literature. Finally, ferroelectric characterization of the BFO matrix was performed using piezoresponse force microscopy (PFM). Out-of-plane PFM measurements were in agreement with results observed previously, but in-plane measurements demonstrated domain wall pinning at the pillar sites. This result is novel in CFO-BFO nanocomposites and merits further exploration. 


\section{Templated $\mathrm{CoFe}_{2} \mathrm{O}_{4}-\mathrm{BiFeO}_{3} \mathrm{Nanocomposites}^{-}$}

In order to be used for practical applications, a means of controlling the growth of $\mathrm{CoFe}_{2} \mathrm{O}_{4}-\mathrm{BiFeO}_{3}$ (CFO-BFO) nanocomposites is required. For the Reconfigurable Array of Magnetic Automata (RAMA) architecture, a square grid of CFO nanopillars with uniform size and precise positioning in a BFO matrix is required. ${ }^{3}$ To develop this capability, templated self-assembly of these nanocomposites using electronbeam lithography to pattern epitaxial CFO islands on the surface of $\mathrm{Nb}$-doped $\mathrm{SrTiO}_{3}(\mathrm{Nb}: \mathrm{STO})$ substrates is investigated. In this chapter, I will discuss the fabrication process, electron and scanning probe microscopy analysis of the nanocomposites that are produced, and the applications of this patterning capability.

\section{Fabrication Process}

A top-down lithrographic process is employed to pattern CFO islands on the substrate surface, followed by a bottom-up self-assembly process to produce the ordered CFO-BFO nanocomposite. A full schematic of the fabrication process is shown in Figure 7-1. In the following sub-sections, detailed analysis of the steps of the fabrication process is discussed. 
a
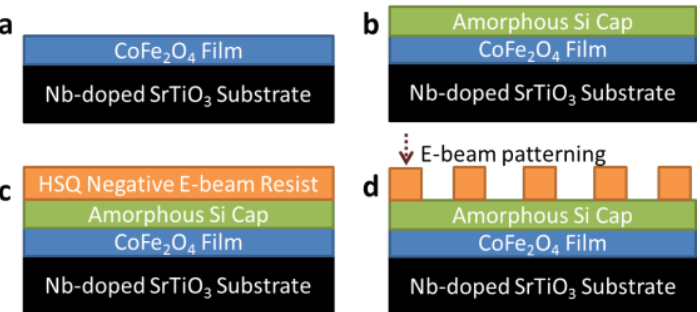

e

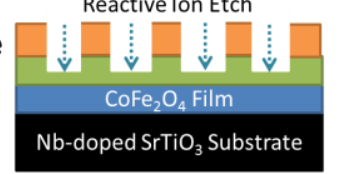

g

$\mathrm{BiFeO}_{3}$ Film Growth

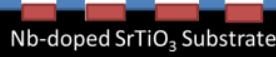

f

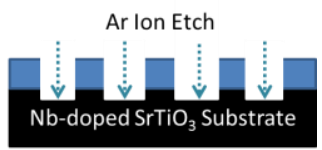

Composite Film Growth

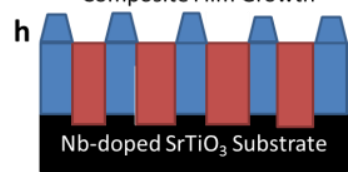

Figure 7-1. Flow chart for the fabrication process. (a) Deposition of $\mathrm{CoFe}_{2} \mathrm{O}_{4}(\mathrm{CFO})$ film using pulsed electron deposition (PED); (b) Deposition of amorphous Si capping layer using RF sputtering; (c) Spin coating of sample with HSQ negative-tone e-beam resist; (d) Patterning of pillars using e-beam lithography; (e) Reactive ion etching of Si cap; (f) Ar ion etching of CFO film; (g) Deposition of $1 \mathrm{~nm}$ thick $\mathrm{BiFeO}_{3}(\mathrm{BFO})$ film using PED; (h) Co-deposition of CFO and BFO using PED to form an epitaxial nanocomposite.

\section{1. $\mathrm{CoFe}_{2} \mathrm{O}_{4}$ Uniform Film}

A $0.5 \%-\mathrm{Nb}$-doped STO (Nb:STO) (001) conductive substrate was prepared using common etching and annealing techniques to produce a $\mathrm{TiO}_{2}$ terminated surface with step-edges due to substrate miscut. ${ }^{58}$ A 12-nm CFO film was then deposited on the substrate using pulsed electron deposition (PED). ${ }^{112,179}$ The CFO film grows via the Volmer-Weber epitaxial growth mode, producing epitaxial islands on the surface. ${ }^{49}$ An atomic force microscopy (AFM) surface topography scan after the growth is shown in Figure 7-2. The sample was found to have islands uniformly coating the surface with diameters between 25 and $50 \mathrm{~nm}$. This result is in agreement with the observations presented in Chapter 5 for uniform CFO films grown on $\mathrm{SrTiO}_{3}$. 


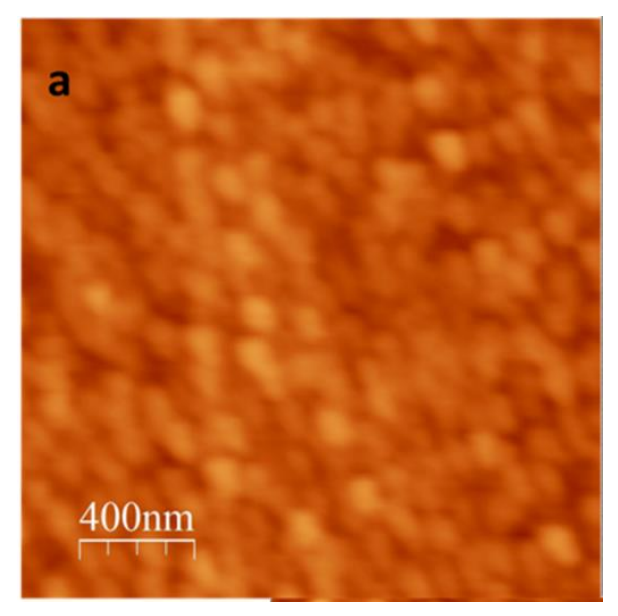

Figure 7-2. Atomic force microscope (AFM) image of uniform CFO film used for template pattern showing regular epitaxial islands on the surface.

\section{Si Capping Layer}

To serve as a capping layer, $\mathrm{Si}$ was deposited onto the $\mathrm{CFO}$ uniform film via radio frequency magnetron sputtering. Si is used to promote adhesion with the HSQ resist and then as a sacrificial etch mask during the reactive ion etching process. ${ }^{125}$ The sputtering deposition parameters were described in Chapter 3. Given that the deposition was performed at room temperature, the Si layer is expected to be amorphous following deposition. To test this, $\mathrm{x}$-ray diffraction and $\mathrm{x}$-ray reflectivity measurements were performed on a sample prior to patterning and etching. Out-of-plane diffraction data and a fit of the x-ray reflectivity measurement are shown in Figure 7-3. The Si film thickness was found to be approximately $25 \mathrm{~nm}$, with an underlying CFO film thickness of approximately $13 \mathrm{~nm}$. These values are in agreement with the calibrations for both deposition rates. The diffraction measurements show the strong STO (001) and (002) substrate diffraction peaks, along with a small CFO (004) film peak, with no other peaks present. These results indicate that the Si film is amorphous. Atomic force microscopy measurements (not shown) have also demonstrated that the Si capping layer conformally coats the CFO island grains on the surface. 
A

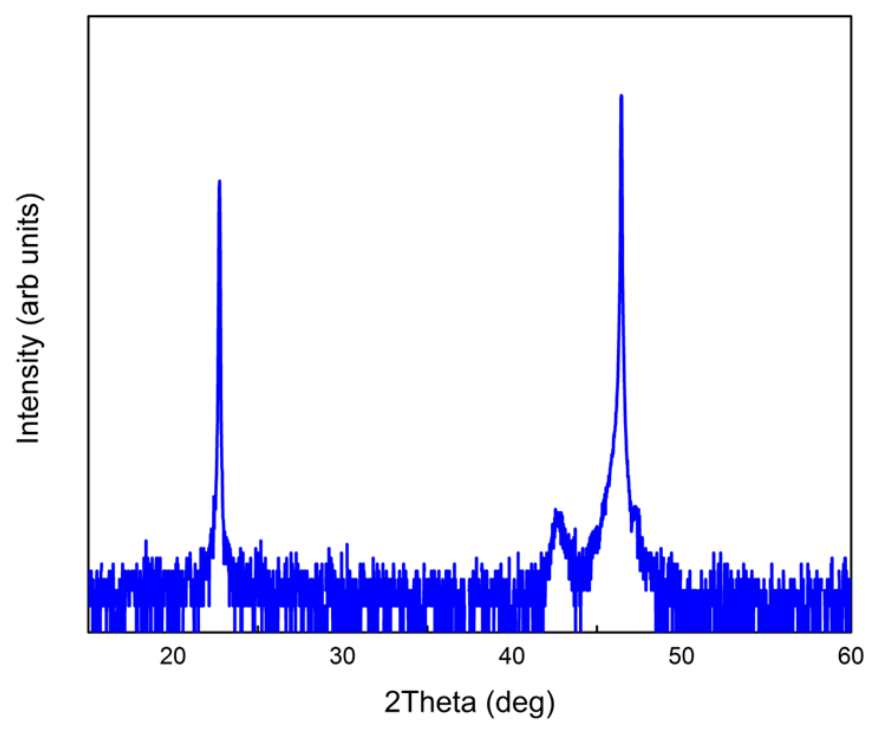

B

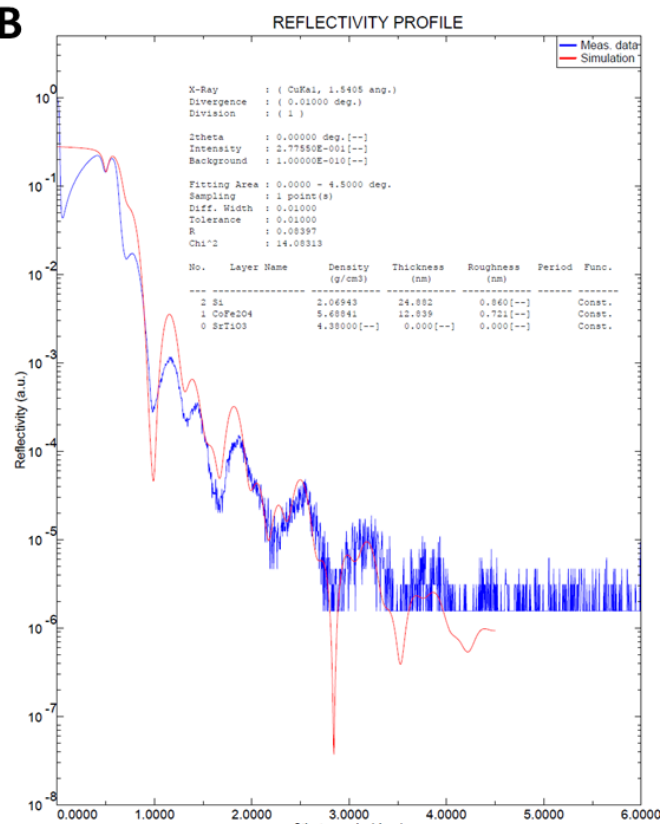

Figure 7-3. (A) Out-of-plane x-ray diffraction data for $\mathrm{Si} / \mathrm{CoFe}_{2} \mathrm{O}_{4} / \mathrm{Nb}: \mathrm{SrTiO}_{3}$ heterostructure. The peak at $\sim 43^{\circ}$ is the CFO (004) peak, while the other two peaks are substrate peaks. (B) X-ray reflectivity data for same structure.

\section{Electron Beam Lithography}

The electron-beam lithography (EBL) fabrication process was outlined in Chapter 3 of this work, with information on the hyrdrosilsesquioxane (HSQ) negative tone resist coating, post-exposure development and optimal doses to produce the patterns. The patterning of pillars in the HSQ resist was achieved with pitch resolution of better than $100 \mathrm{~nm}$ between the center of neighboring pillars. While it was possible to produce smaller pitch sizes during the EBL process, attempts to transfer the pattern through the $\mathrm{Si}$ capping layer and CFO film during the reactive ion etching (RIE) failed.

An example of a $100 \mathrm{~nm}$ pitch array is shown below in Figure 7-4. A uniform array of pillars with diameter slightly less than $50 \mathrm{~nm}$ is observed with the island grains from the underlying Si-capped CFO film visible in the area not covered by the pillars. It is worth noting that the diameter of the pillars is at least as large as the diameter of the CFO islands in the film below, so it is likely that some or all of the patterned islands will be patterned across the boundaries of multiple islands. This non-uniformity in the island template could cause the various sizes and shapes of nanopillars observed via transmission electron 
microscopy later in this chapter. Atomic force microscopy (not shown) indicated that the pillars were generally between 40 and $50 \mathrm{~nm}$ in height, with some variation both within the arrays and between samples. In terms of pattern fidelity, it was found that the area density of the pillars and the proximity of the pillar to the edge of the array affected the diameter of the pillar following development. This can be attributed to unintended electron exposure of resist outside of the pattern area via secondary and backscattered electrons during the exposure of a patterned pillar. Thus, pillars in the center of the array will receive a higher dose than those near the edge of the array, producing larger pillar heights and diameters. This effect is known as the proximity effect and is well understood in the EBL community. ${ }^{180}$ Techniques exist to correct for this but were not pursued in this work.

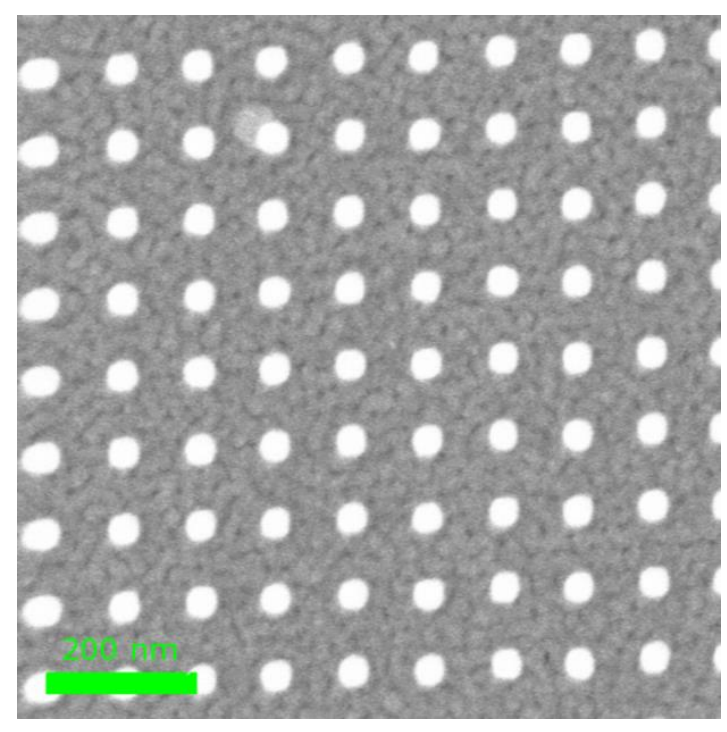

Figure 7-4. Scanning electron microscope image of EBL-patterned HSQ pillars on the surface of the Si capping layer. Scale bar represents $200 \mathrm{~nm}$.

\section{Reactive Ion Etching}

Following the EBL process, reactive ion etching (RIE) was employed to transfer the pattern of HSQ pillars through the Si capping layer and CFO film to produce template islands on the surface of the substrate. A description of the process was given in Chapter 3 of this work. An example of the resulting topography is shown in Figure 7-5. This image was taken at the edge of the patterned array, allowing for 
clear inspection of the substrate surface quality. It is clear that unit cell terraces are present on the surface of the substrate following the etching process, indicating that the surface quality is ideal for the growth of an epitaxial film. The observation of these step edges, along with a smooth terrace showing no evidence of the original CFO island grain structure, was used to verify the completion of the RIE process. A few defect areas in the array are observed, which is the result of particulate that was deposited on the surface during the growth of the initial CFO film. The density of this particulate varied from sample to sample but was generally not an impediment to the overall fabrication process. This particular template sample was not used for any other results presented in this thesis, but is representative of the surface morphology observed for all templates used. The height of the CFO islands after patterning was approximately 5 for most samples, as measured via AFM. An AFM image of the array of pillars characterized via electron microscopy, along with a profile of the pillar height is shown in Figure 7-6. The measurement indicates that the pillar height is between 4 and $5 \mathrm{~nm}$ for this sample. Templating was generally not successful for islands that were smaller than this height. There is no direct means to characterize the depth of etching into the substrate without the use of cross-sectional transmission electron microscopy (TEM), which was not employed. The effect of any substrate etching, along with the thickness of the CFO portion of the island will be discussed later in this chapter during TEM analysis of a nanocomposite grown on the etched template. 


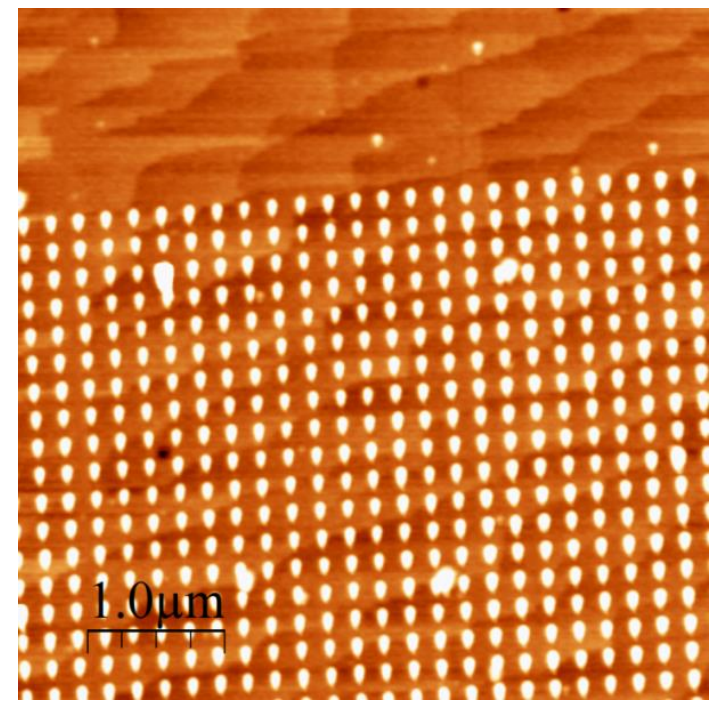

Figure 7-5. Atomic force microscope topography image of island array with $200 \mathrm{~nm}$ pitch after RIE showing atomic terraces on substrate surface.
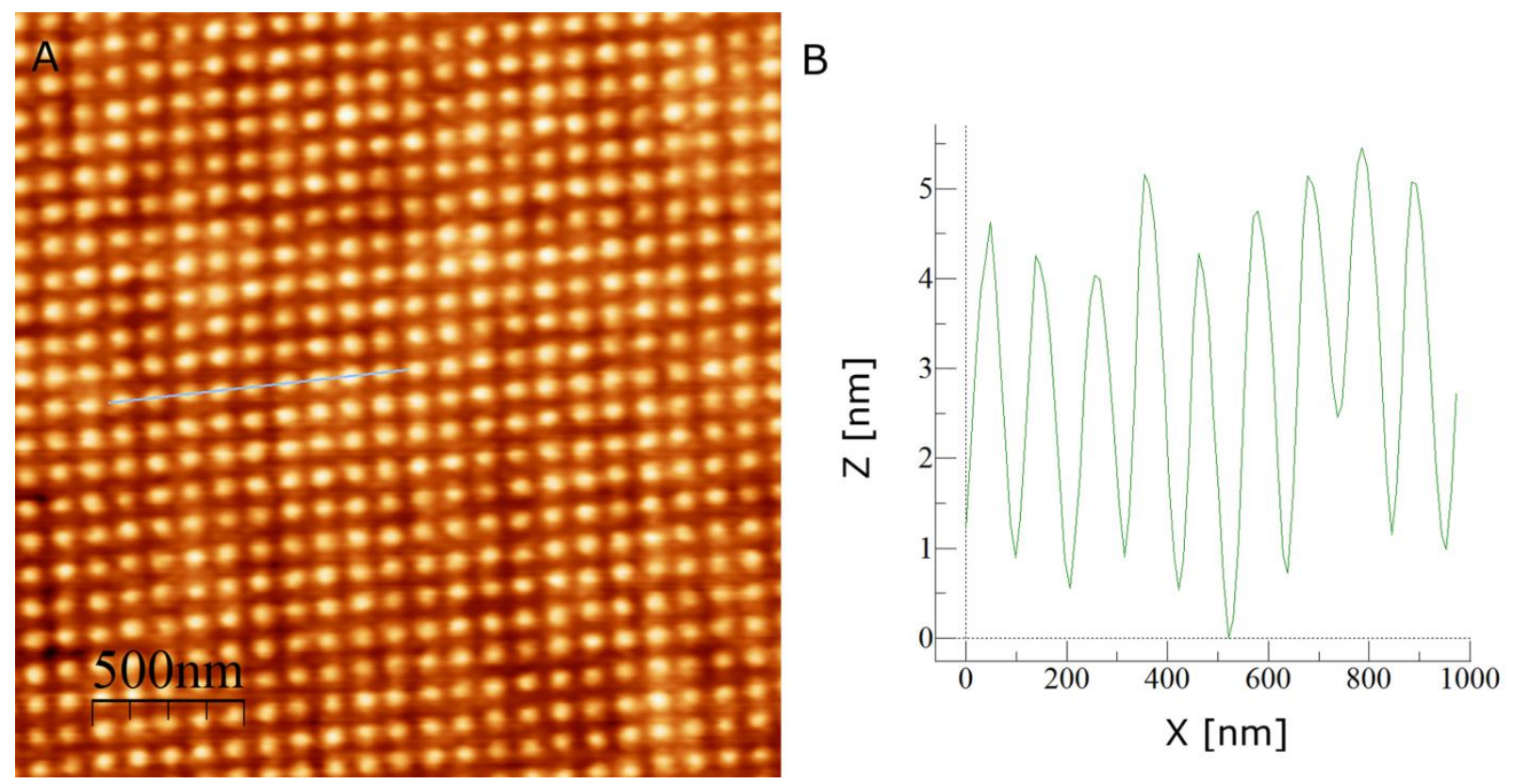

Figure 7-6. Array of template islands patterned for sample characterized via electron microscopy. A) Atomic force microscopy topography image of array; B) Height profile along gray line in (A). 


\section{Structural Characterization of Templated Composites}

To characterize the structural and chemical properties of the patterned nanocomposite, a sample with calibrated BFO matrix thickness of $25 \mathrm{~nm}$ was grown on the template substrate shown in Figure 7-6 with a pillar pitch of $100 \mathrm{~nm}$. The growth conditions were chosen to match those of the unpatterned sample characterized via transmission electron microscopy (TEM) in Chapter 6. This sample was then characterized via x-ray diffraction, scanning electron microscopy and cross-sectional TEM. Vector-mode piezoresponse force microscopy measurements were also made prior to the electron microscopy analysis and are presented later in this chapter.

\section{X-Ray Characterization}

The sample was characterized via x-ray reflectivity (XRR), out-of-plane x-ray diffraction (XRD) and a reciprocal space map (RSM) about the Nb:STO (103) peak. Due to the small area coverage of the template across the entire area of the substrate $(10 \mu \mathrm{m} \times 10 \mu \mathrm{m}$ array on a $5 \mathrm{~mm} \times 5 \mathrm{~mm}$ substrate), the measurements are based almost entirely on the structural properties of the film away from the patterned region. Electron microscopy or synchrotron nanodiffraction would be required to examine the structural properties of the patterned arrays. The XRR measurement confirmed that the thickness of the matrix was approximately $24.9 \mathrm{~nm}$, matching well with the calibrated growth rate. XRD measurements were used to determine the out-of-plane lattice parameter of the BFO film, which was found to be $4.07 \AA$. The lattice parameter of any CFO pillars could not be determined due to the low area volume density of the CFO phase and the close overlap between the CFO (004) diffraction peak and the BFO (002) $)_{\mathrm{pc}}$ diffraction peak. The RSM, which is shown in Figure 7-7, indicated that the BFO film was coherent with the Nb:STO substrate along the in-plane direction. The reciprocal lattice coordinate, $Q_{x}$, represents the diffraction peak along the [100] in-plane direction, while the $Q_{z}$ coordinate represents the out-of-plane [001] direction. Both the BFO and Nb:STO peaks fall at the same value of $Q_{x}$, indicating coherent strain at the interface. 


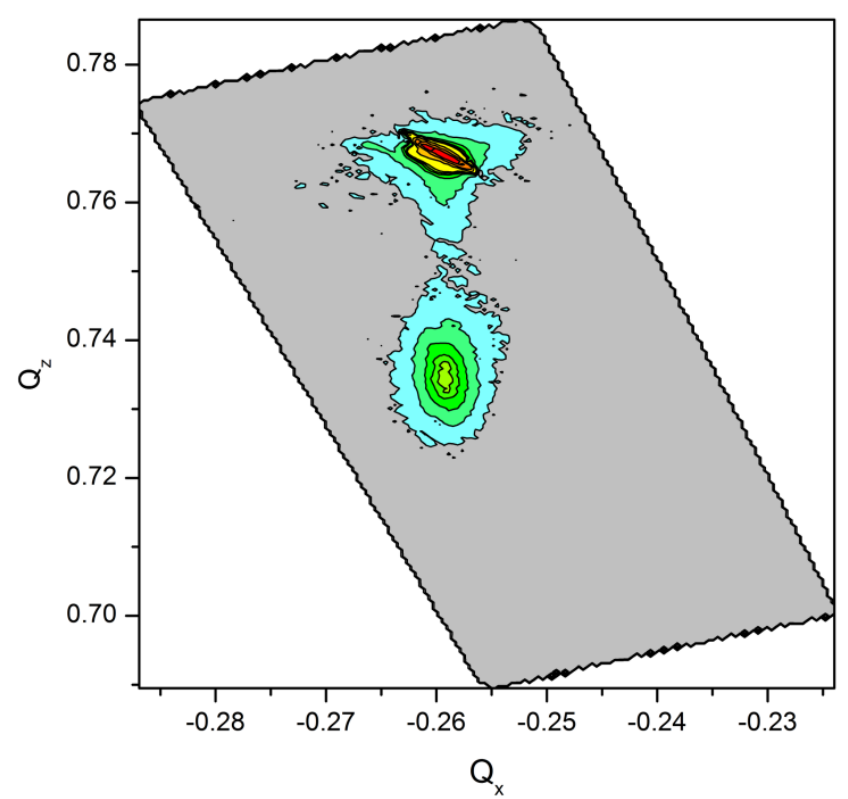

Figure 7-7. Reciprocal space map of sample showing the (103) diffraction peak of the $\mathrm{Nb}$-doped $\mathrm{SrTiO}_{3}$ substrate (top) and pseudocubic (103) peak of the $\mathrm{BiFeO}_{3}$ matrix (bottom).

\section{Electron Microscopy}

To characterize the templating effects of the $\mathrm{CFO}$ island on the resulting nanocomposite, the sample characterized by x-ray diffraction was also examined using scanning electron microscopy (SEM), transmission electron microscopy (TEM) and high-angle annular dark field scanning transmission electron microscopy (HAADF-STEM). A single array with pitch of $100 \mathrm{~nm}$ along the [100] in-plane directions was used for all measurements. These measurements were useful to determine the epitaxial configuration of the pillars, which are expected to have $\{110\}$-type in-plane facets with the BFO matrix. ${ }^{71}$ This was confirmed for the unpatterned samples examined in Chapter 6. For TEM measurements, a crosssectional sample was extracted using a dual-beam focused ion beam-scanning electron microscope system with orientation along the $\langle 110\rangle$ substrate axis. Details of this process are discussed in Chapter 4.

An SEM image of the array is shown in Figure 7-8. The horizontal axis of the figure is aligned to correspond to the [100] substrate axis. The pillars are clearly facetted along the $\{110\}$ planes, indicating an epitaxial interface with the BFO matrix. There is some variation in pillar size from site to site, but there is $100 \%$ pattern fidelity within the image. Over larger regions of the array, which is a 100x100 array 
covering a $10 \mu \mathrm{m}$ by $10 \mu \mathrm{m}$ area, there are some defective regions that are most likely the result of the deposition of particulate during the PED growth process.

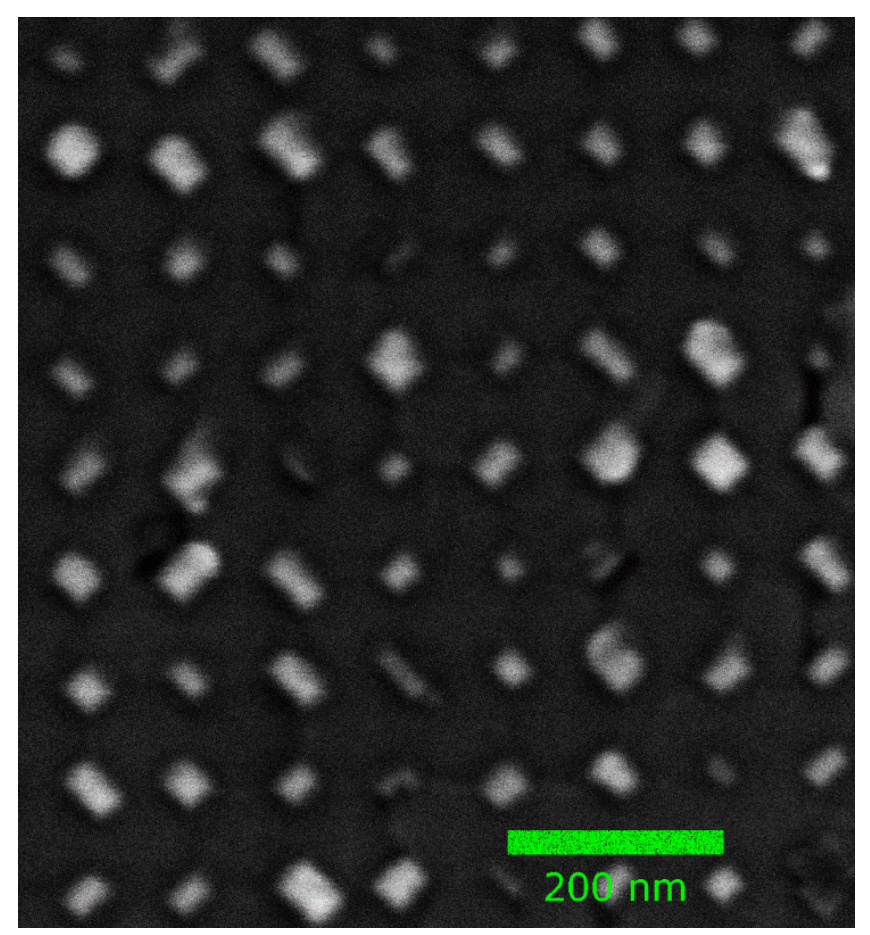

Figure 7-8. Scanning electron microscope image of templated array of $\mathrm{CoFe} 2 \mathrm{O} 4$ nanopillars in $\mathrm{BiFeO3}$ matrix with $100 \mathrm{~nm}$ pitch. Horizontal axis is parallel to [100] direction.

After characterizing the plan-view morphology using SEM, a cross-sectional lamella was extracted along the [110] diagonal and examined using a FEI Titan 80-300 TEM and STEM system. The sample was cut along the diagonal to produce a more uniform view of the CFO pillars. Had the cut been made along the [100] axis, the thickness of the CFO pillar would vary in the image and make analysis more difficult. HAADF images of two template pillars that were characterized in detail are shown in Figure 7-9. The spacing between the center of the pillars is approximately $140 \mathrm{~nm}$, which is approximately equal to $\sqrt{2} * 100 \mathrm{~nm}$, the spacing that results from extracting the sample along the diagonal. Two additional pillars are visible to the left of the primary two shown in Figure 7-9(A), but are more faint. The low contrast of these two pillars is likely the result of the cross sectioning process hitting a smaller portion of the pillar, reducing the contrast with the BFO. The sensitivity of HAADF measurements to the atomic 
number, $Z$, means that $\mathrm{Bi}$, which has atomic number 83 will produce the brightest contrast, while $\operatorname{Sr}(Z=$ 38), $\mathrm{Ti}(Z=22), \mathrm{Co}(Z=27)$, and $\mathrm{Fe}(Z=26)$, will be fainter. There is a brighter contrast around the portion of the pillar that is below the surface of the matrix, which can be attributed to residual BFO in front of or behind the pillar. The thickness of the cross-section sample is estimated to be between 50 and $75 \mathrm{~nm}$, which is greater than the width of the pillar shown in the SEM image in Figure 7-8.

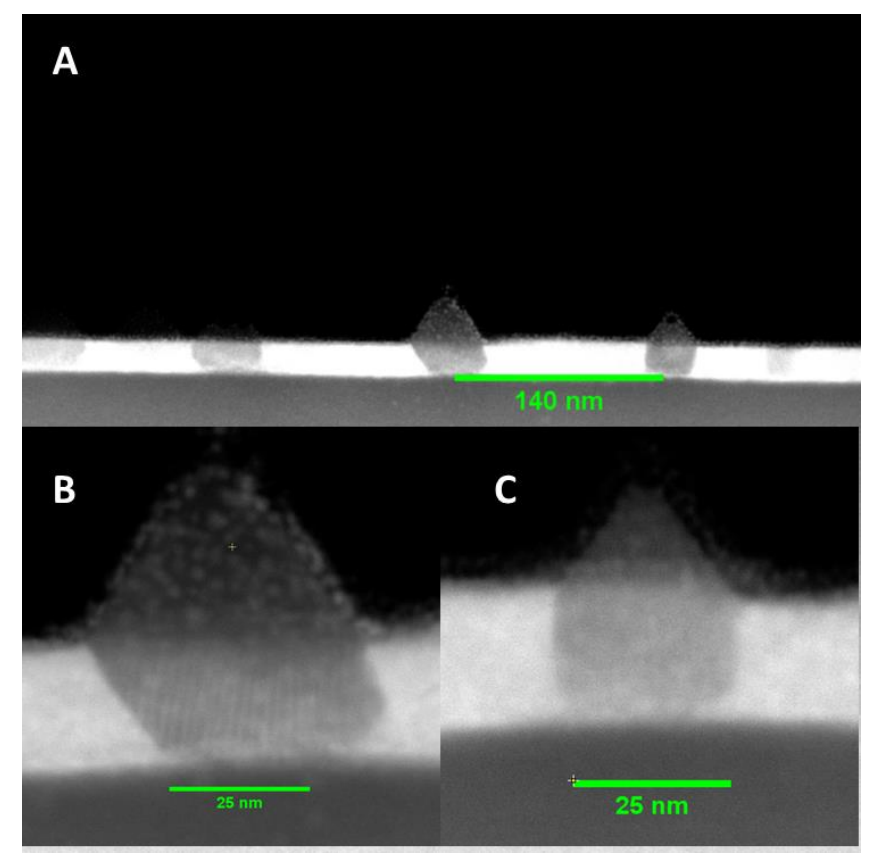

Figure 7-9. HAADF-STEM image of patterned nanocomposite. A) Wide image showing uniform spacing of pillars; B) Image of left pillar in (A); C) Image of right pillar in (A).

High resolution HAADF images of the two pillars of interest are shown in Figure 7-9(B-C). The base of the pillar is approximately $25 \mathrm{~nm}$ in width in both cases and $\{111\}$-facets are visible above the surface of the matrix, which was confirmed by measurements of the angle between the facet and the horizontal matrix surface. Interestingly, the interfaces beneath the surface for the two pillars are very different. In Figure 7-9(B), the pillar tilts to one side, while in Figure 7-9(C) the pillar is vertical. This can most likely be attributed to the difference in nucleation behavior at the surface of the CFO island that was patterned on the substrate. To further understand the nature of the interface, energy dispersive x-ray spectroscopy 
(EDS) maps were taken for both pillars. In these measurements, a spectrum of x-ray energies is obtained across a uniform grid of points using the STEM mode of the Titan microscope.

The results of the EDS map for the pillar shown in Figure 7-9(C) are shown in Figure 7-10, with the acquired HAADF STEM image (A), green map (B) corresponding to the Bi $L_{\alpha}$ peak, the red map (C) corresponding to the $\mathrm{Co} K_{\alpha}$ peak, and the blue map (D) corresponding to the Ti $K_{\alpha}$ peak. The peak was acquired over a grid with $2 \mathrm{~nm}$ It should be noted that the Co $K_{\alpha}$ peak is very close in energy to the Fe $K_{\beta}$ peak, meaning that the meaning that the peak at the $\mathrm{Co} K_{\alpha}$ location attributed to the BFO matrix will be non-zero as a result of the Fe present in the matrix. Additionally, the presence of the BFO in front of or behind the pillar means that the Bi map will also have significant intensity in the pillar region. To clarify the results, image processing was performed so that lower intensity regions were partially transparent and a single image with all color maps overlaid was created. The result is shown in Figure 7-11(A). An additional EDS map was also collected with $1 \mathrm{~nm}$ resolution at the interface between the pillar and substrate to examine the nucleation effects. The same overlay procedure was performed and the results of this map are shown in Figure 7-11(B). 


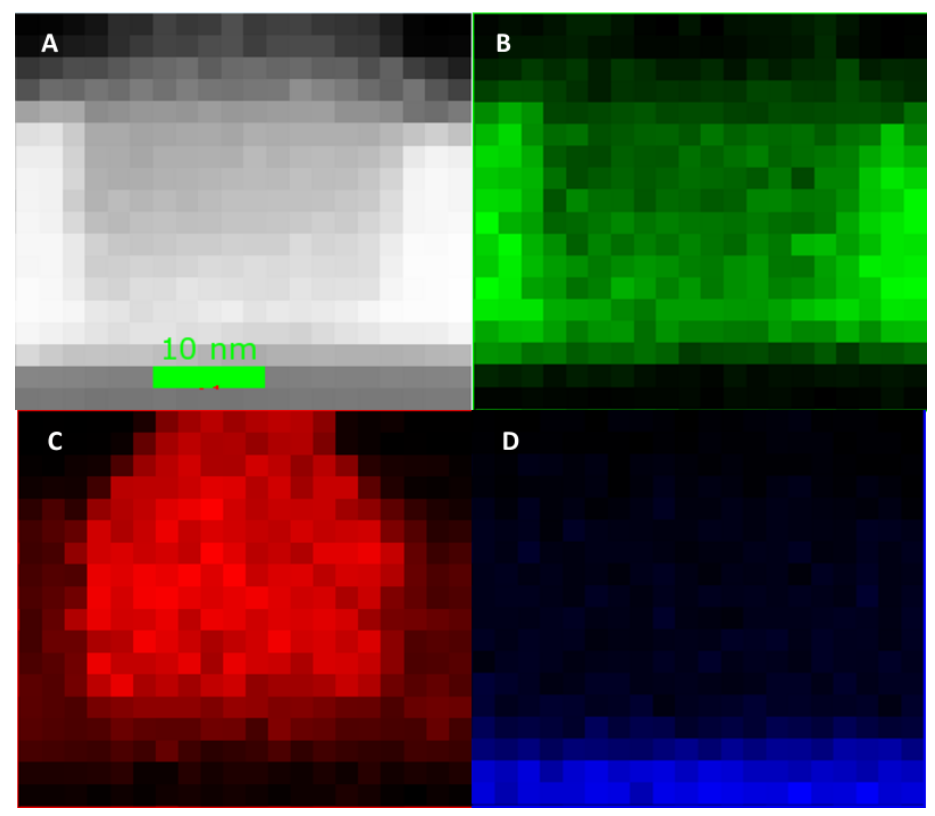

Figure 7-10. Energy dispersive x-ray spectroscopy map of pillar from Figure 7-9(C). A) HAADF signal at each pixel measured; B) Bi $L_{\alpha}$ edge map; C) Co $K_{\alpha}$ edge map; D) Ti $K_{\alpha}$ edge map.

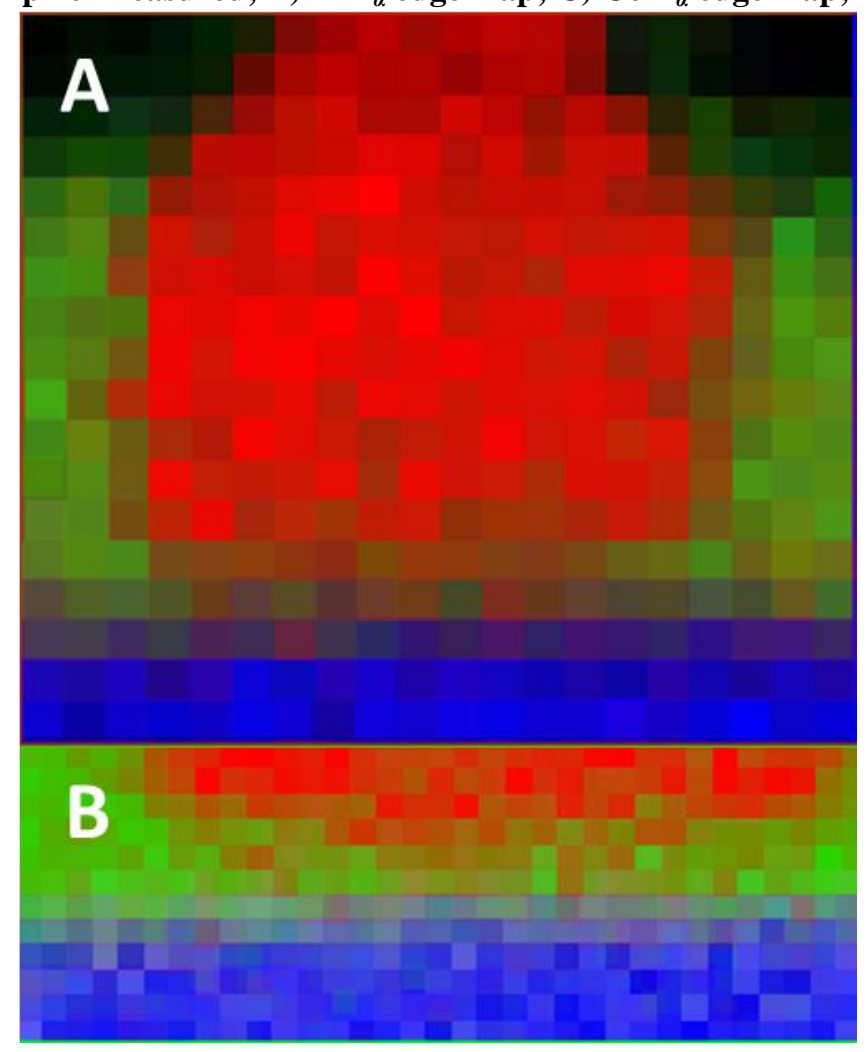

Figure 7-11. A) Overlay of EDS map images from Figure 7-10. b) Overlaid images from high resolution EDS map of pillar-substrate interface. 
Analysis of the maps in Figure 7-11 is enlightening to explain the growth process and supports the hypothesis for the chemical nature of the pillar templating. The Ti map was relatively smooth, with no apparent mound of $\mathrm{SrTiO}_{3}$ present beneath the pillar. This indicates that the reactive ion etching process likely removed at most $1 \mathrm{~nm}$ of the substrate based on the pixel size chosen for the scan, leaving at least 3 nanometers of CFO on the surface of the island to serve as a chemical template. Further analysis of the interface between the substrate and template island will be presented below with TEM images of the lattice. The Bi map shows that there is a greater Bi density within $5 \mathrm{~nm}$ of the substrate than in the areas farther away. This can likely be attributed to two features of the growth: the initial $1 \mathrm{~nm}$ layer of BFO deposited to coat the surface of the substrate, and the outgrowth of the pillar as it increases in height. The initial BFO layer may partially overcoat the edges of the CFO island, producing a greater density of BFO around the edge. Additionally, the narrow base of the CFO pillar means that there would be additional BFO along the beam path in this region.

Further analysis of this pillar was performed using high-resolution TEM. A series of TEM images of the pillar at various magnifications is shown in Figure 7-12. As in the unpatterned composite analyzed in Chapter 6, these images show the presence of Moire fringes at due to the incoherent interface between the CFO pillar and BFO matrix. The Moire fringing pattern, however, is somewhat less uniform than in the case of the spontaneously formed pillar examined. It is possible that the strain interactions in the pillar are more complex due to the presence of the template island. Further analysis of the effect of the template island on the pillar morphology and strain is required. The substrate appears to be fairly smooth, with a slight rise near the pillar. This could be attributed to overetching during the RIE step in the fabrication process. As in the EDS map, however, any rise is no more than 1-2 $\mathrm{nm}$ in height, indicating that there was still residual CFO in the island template. This confirms that the islands act to chemically control the growth of the CFO pillars, rather than simply to promote the growth of the pillars due to the topographic templating, as observed in focused ion beam templating of Ge quantum dots. ${ }^{96}$ 


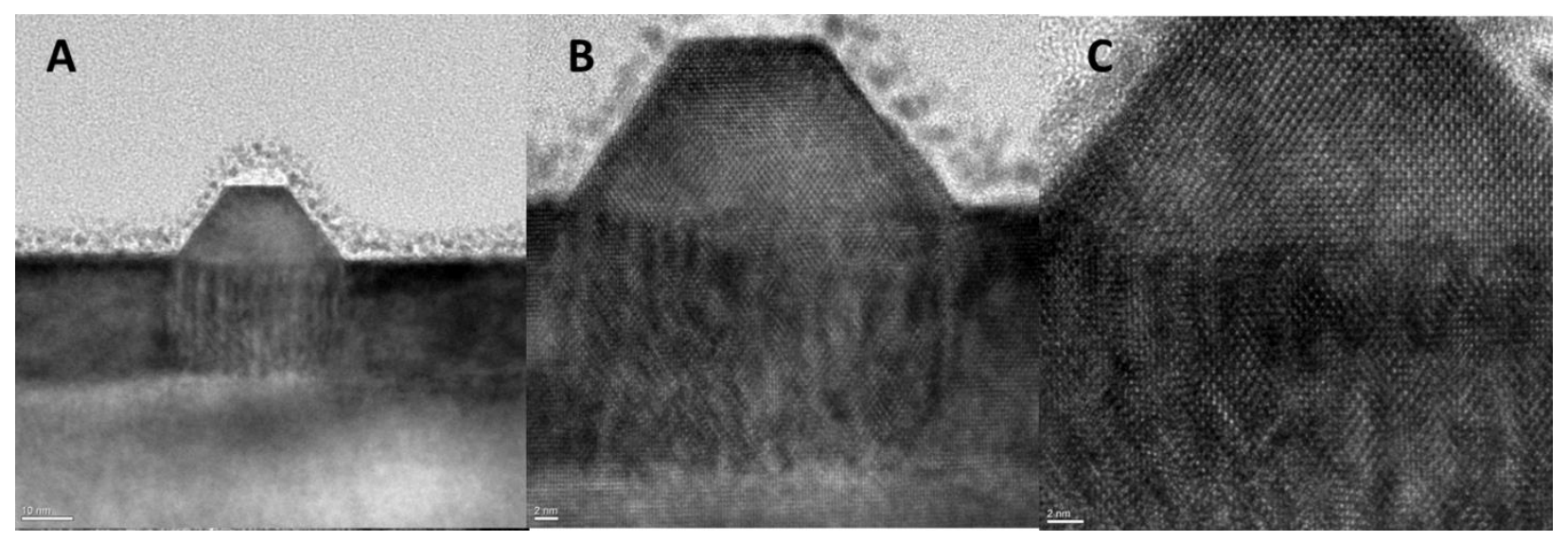

Figure 7-12. TEM images of pilar from Figure 7-9(C). (A) Wide view (scale bar represents $10 \mathrm{~nm})$; (B) Tight view showing Moire fringing due to incoherent pillar-matrix along zone axis (scale bar represents $2 \mathrm{~nm}$ ); (C) View of pillar facet above surface of matrix (scale bar represents 2 nm).

To characterize the strain in the pillar, FFT analysis was performed on several high magnification images acquired at 380k magnification. Real space images, along with filtered images used to analyze the coherency of the interfaces are shown in Figure 7-13. For the pillar-substrate interface, horizontal line profiles were acquired in the filtered image (Figure 7-13(D)) and the number of unit cells in both the pillar and substrate over a $10 \mathrm{~nm}$ window were counted to determine the strain at the interface. For the $\mathrm{Nb}$ :STO substrate, 36 peaks were observed over $10 \mathrm{~nm}$, which is in agreement with the $3.905 \AA$ lattice parameter expected for STO. Depending on the window chosen, either 33 or 34 CFO peaks occur every $10 \mathrm{~nm}$, which is in agreement with a fully incoherent interface. It is impossible to measure small residual strains to sufficient precision to predict magnetic anisotropy using this approach. Analysis of the FFT patterns, shown in Figure 7-14 and Figure 7-15 also produces uncertainties too large to determine the inplane lattice parameter of the CFO pillar to sufficient precision as to predict the anisotropy. Similar measurements were made on the BFO-CFO vertical interface and showed that the dislocation density at the interface was in agreement with a fully relaxed interface. The FFT patterns shown match those of the unpatterned composite presented in Chapter 6, further indicating that the sample has the expected crystal structure. 


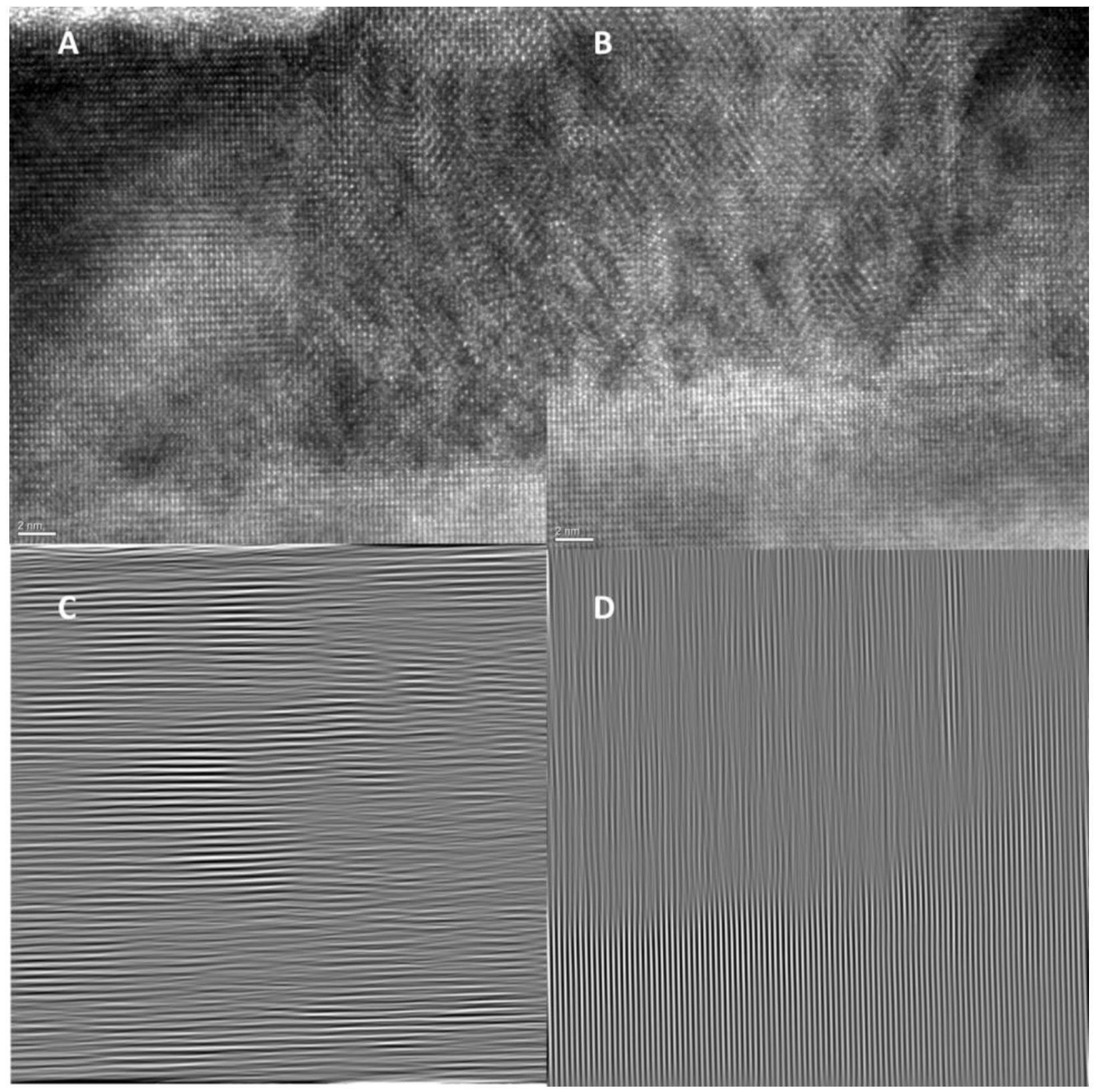

Figure 7-13. HRTEM images of matrix-pillar (A) and pillar-substrate (B) interfaces. C) Fourier-filtered image of (A) used to examine coherency of matrix-pillar interface. D) Fourier-filtered image of (B) used to examine pillar-substrate interface. Scale bars in (A) and (B) represent 2 nm. 


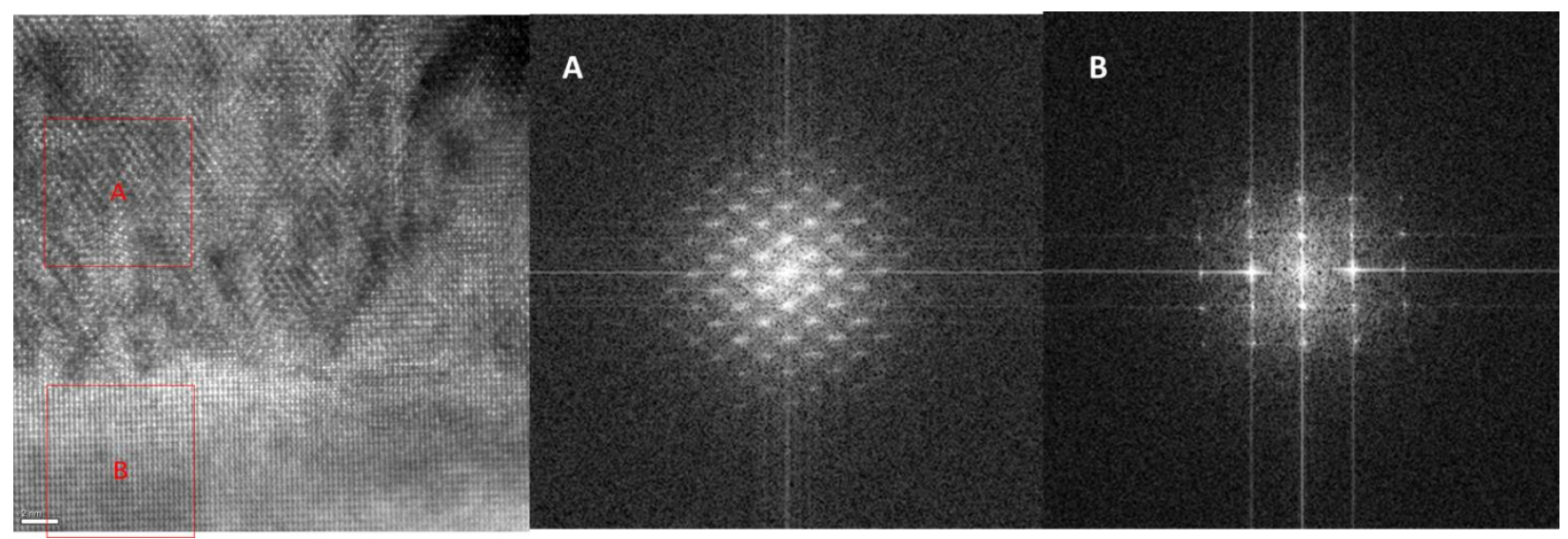

Figure 7-14. FFT analysis of pillar-substrate interface, with regions of interest outlined in the image to the left. A) FFT image of pillar; B) FFT image of substrate.
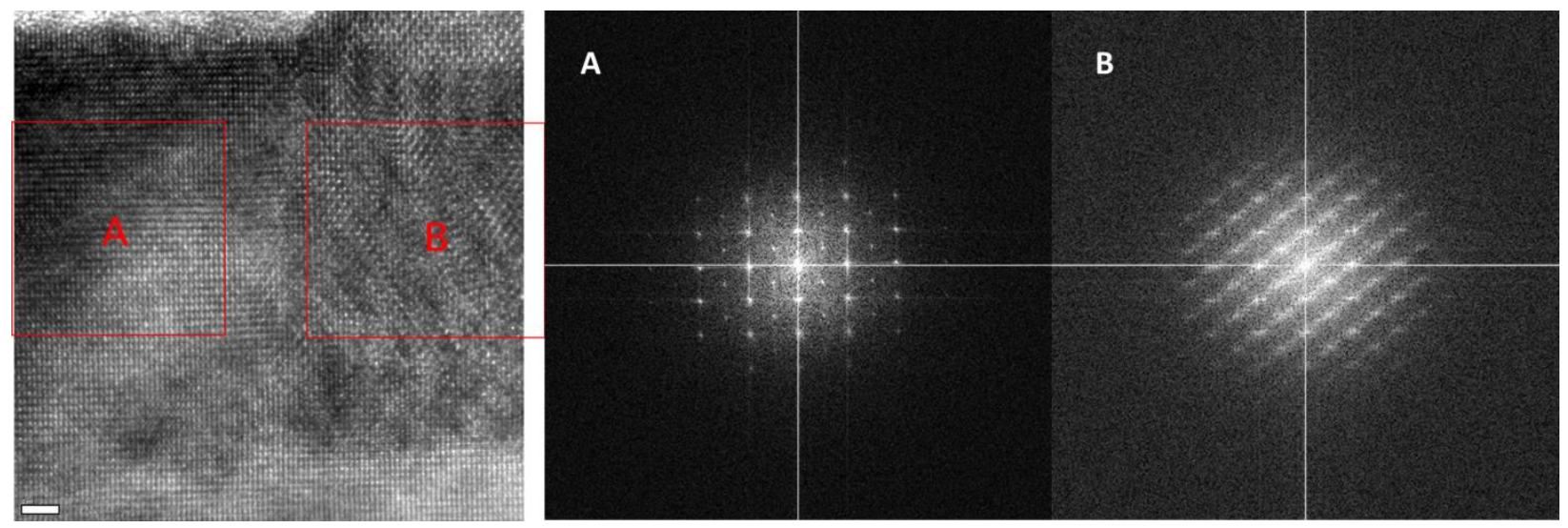

Figure 7-15. FFT analysis of pillar-matrix interface, with regions of interest outlined in the image to the left. A) FFT analysis of matrix; B) FFT analysis of pillar.

While the pillar presented above shows what might be considered the ideal geometry, it is also worthwhile to examine other pillars with more unusual microstructure. A series of TEM images of a single pillar at various magnifications is shown in Figure 7-16. In Figure 7-16(B), it is clear that the patterned island produced some overetching into the substrate, as an $\sim 3 \mathrm{~nm}$ tall island of $\mathrm{Nb}: \mathrm{STO}$ is visible at the base of the CFO pillar. Based on the AFM analysis presented in Figure 7-6, which showed that the islands for this sample were between 4 and $5 \mathrm{~nm}$ in height, it is likely that some CFO phase was still present on the top of the island after etching. This would support the notion that the templating effect 
is primarily chemical rather than topographic. An additional HRTEM image, along with associated color coding of the phases present is shown in Figure 7-17. In Figure 7-17(A), there is a dark region visible at the interface between the STO island and the CFO pillar. This could be attributed to a region of high defect density due to the etching process. However, the resulting CFO pillar shows good crystal quality above the interface in spite of the non-ideal template island. The Moire fringes are more pronounced than in the previous pillar, which could be the result of a thicker BFO layer along the beam axis.

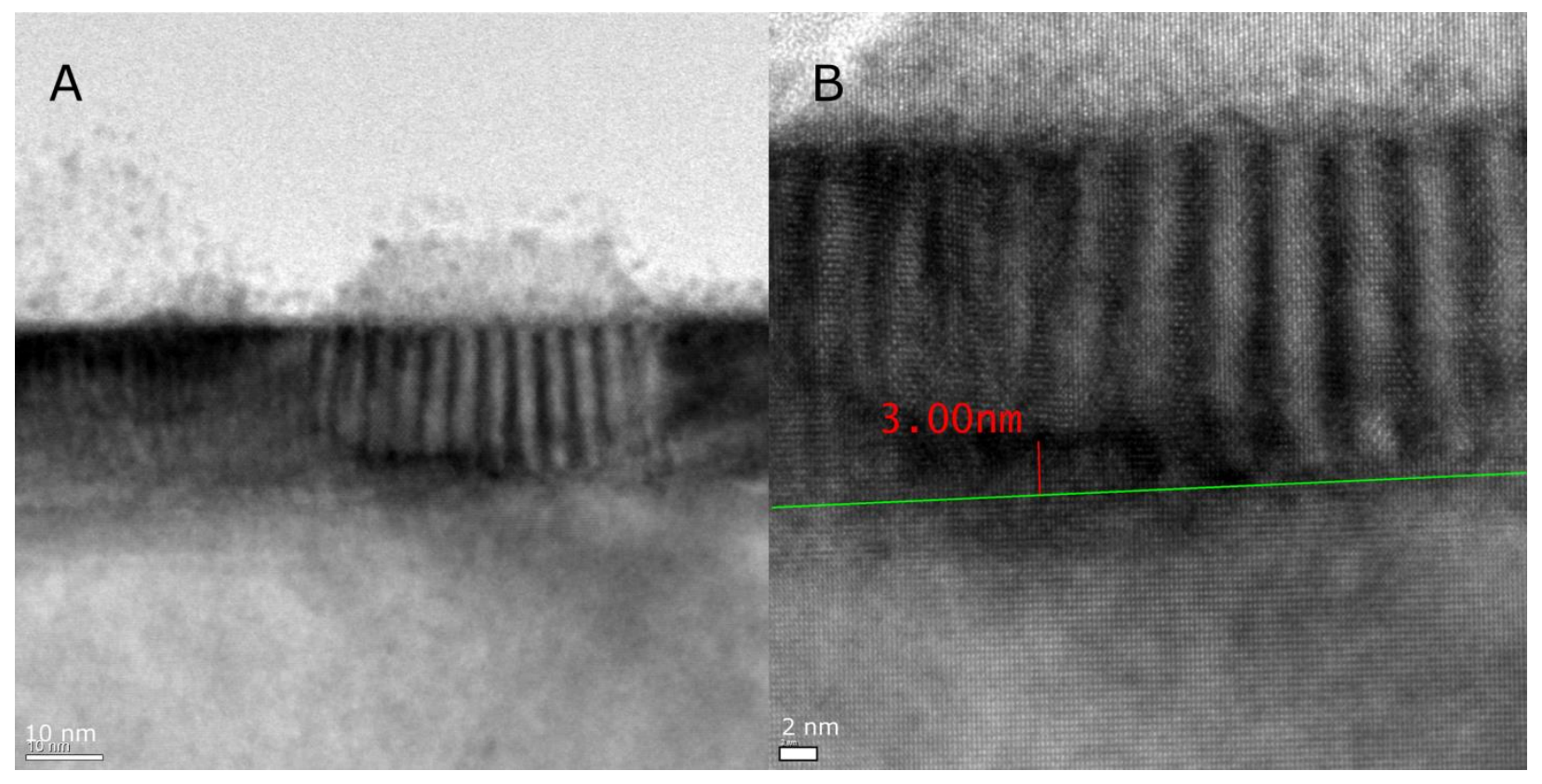

Figure 7-16. High-resolution TEM images at two diffrent magnifications showing a pillar formed on top of an island comprised of some of the Nb:STO substrate. A) 115,000x magnfication; B) 245,000x magnfication with annotations showing the substrate surface (green) and $3 \mathrm{~nm}$ tall $\mathrm{Nb}: \mathrm{STO}$ mound (red) that is formed beneath the CFO island. 


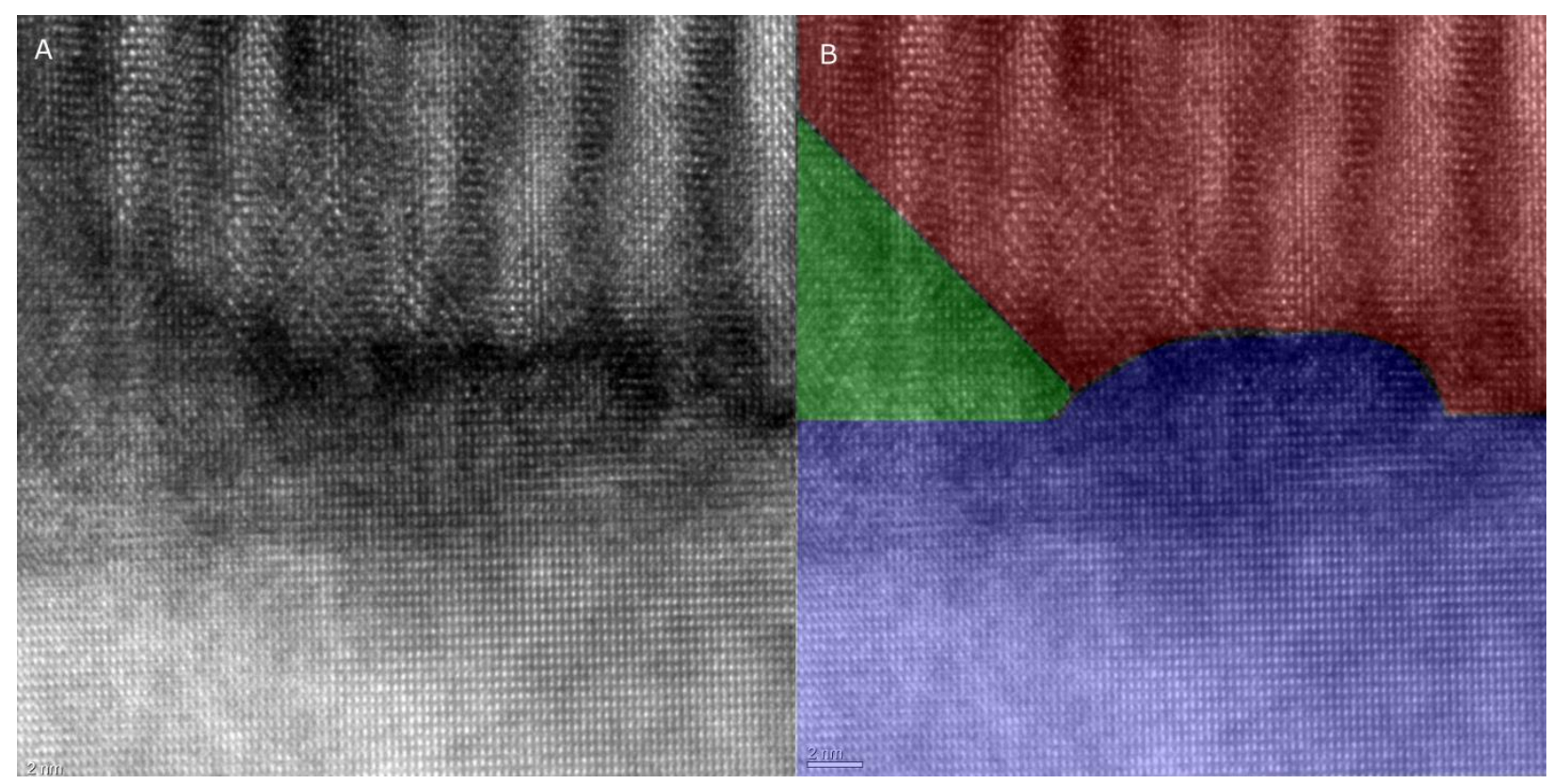

Figure 7-17. High-resolution TEM image at 380,000x magnification of the same pillar as BLAH (A), with false color overlay $(\mathrm{B})$ showing the estimated interface boundaries between $\mathrm{Nb}: \mathrm{SrTiO}_{3}(\mathrm{blue}), \mathrm{BiFeO}_{3}(\mathrm{green})$ and $\mathrm{CoFe}_{2} \mathrm{O}_{4}$ (red).

\section{Faulty Templating}

While an ideal template can produce arrays of pillars where all CFO flux diffuses to the template sites, a template that is not properly prepared will have very different effects on the growth process. Figure 7-18 shows an SEM image of an array where the template did not have the desired effect. In this case, it is likely that the etching process did not fully expose the STO substrate between the patterned CFO islands. Because of the large lattice mismatch between $\mathrm{CFO}$ and $\mathrm{BFO}$, the epitaxial growth of BFO on top of the residual CFO does not produce the high quality film that can be achieved on a smooth STO substrate. Instead, facetted structures of unknown composition form on the surface. In this particular case, at the corner of the patterned array the substrate was exposed sufficiently to achieve the desired pattern. This result can be attributed to variations in the EBL dosage, where features at the edge of the pattern have a lower effective dose because secondary electron emission from the exposure of neighboring pillars is reduced. 


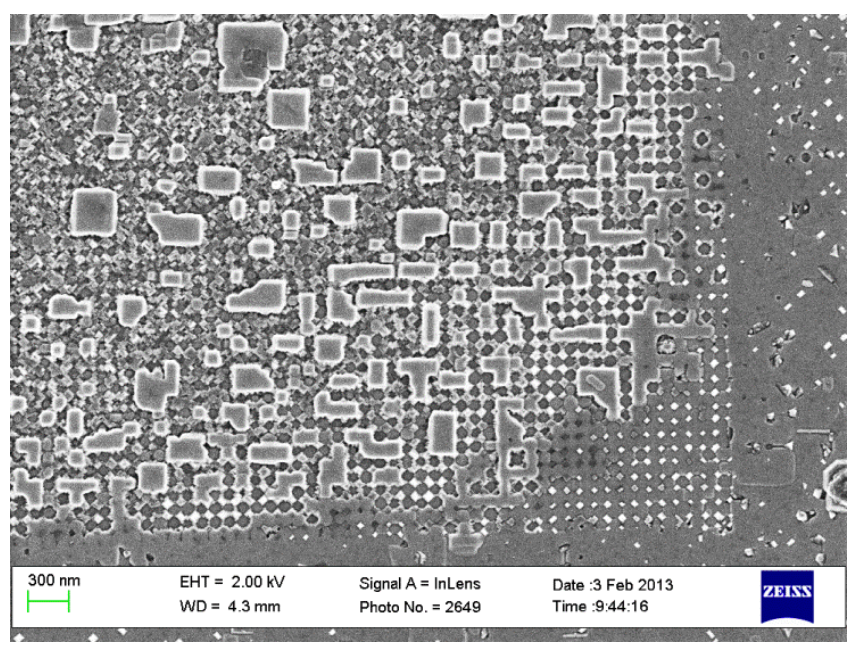

Figure 7-18. Scanning electron microscope image of array exhibiting faulty templating. Note that properly templated pillars form at the corner of the array due to variation in the electron beam lithography process near the edges of the array.

In the case of islands that are smaller than the ideal of $\sim 5 \mathrm{~nm}$, the opposite effect occurs during the growth process. When grown on such a template, no observable templating occurs and CFO pillars form randomly via the spontaneous nucleation process that occurs in unpatterned nanocomposites. This could be attributed to the loss of the CFO phase at the top of the island. As observed in Error! Reference ource not found., if the island has been etched an additional 1-2 nm, it is likely that all CFO on the island would have been removed. Thus, a topographic variation from the presence of a mound of $\mathrm{Nb}: \mathrm{STO}$ on the substrate surface appears to be insufficient to promote the nucleation of a pillar.

\section{Growth Kinetics}

To characterize the effect of different pitch sizes on the templating effects of the islands, three different arrays of different pitches were patterned onto the same substrate and a nanocomposite film was grown on the sample. An SEM image showing the pattern is visible in Figure 7-19. Slight astigmation is present in the image, making the arrays appear to contain lines rather than pillars, which is an artifact of the measurement. Higher resolution images such as the one presented in Figure 7-4 confirm this. By 
preparing this template with three different pitch sizes, it is possible to examine the effects of surface diffusion and growth kinetics on the templating process. ${ }^{100}$

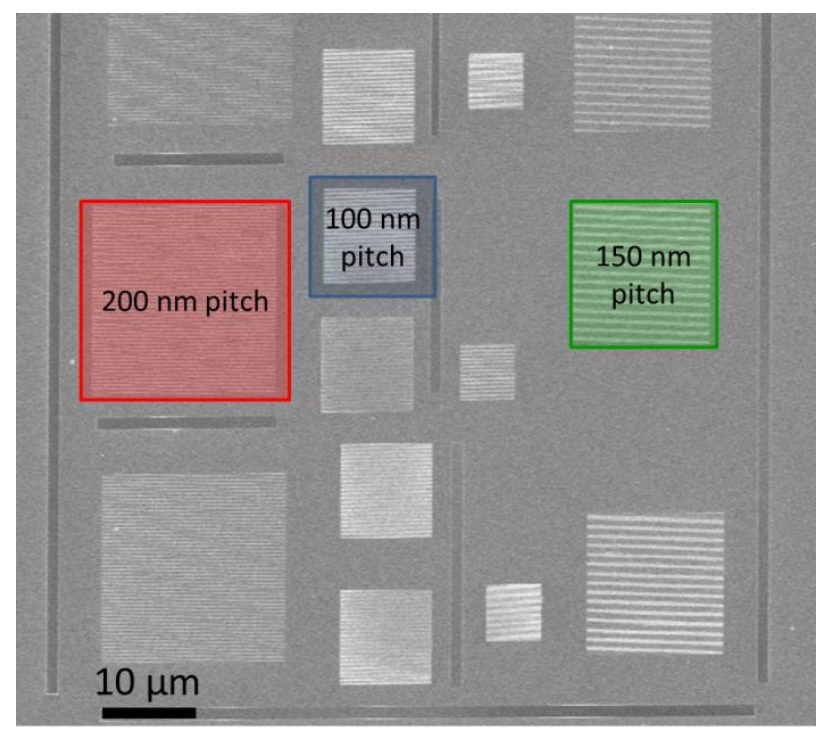

Figure 7-19. Scanning electron microscope image of arrays with three different pitch sizes patterned on the same substrate.

The resulting film was characterized using an Asylum Research Cypher AFM system. AFM analysis was performed on the arrays with pillar pitches of 100, 150 and $200 \mathrm{~nm}$. Images for all three dimensions are shown in Figure 7-20, along with cross-sectional height data for each image. The $100 \mathrm{~nm}$ and $150 \mathrm{~nm}$ pitch arrays have uniformly distributed pillars with no interstitial pillars present. In the $200 \mathrm{~nm}$ arrays some small CFO pillars nucleated in spite of the initial BFO wetting layer, an example of which is highlighted with a diamond in Figure 7-20(c). A key issue is whether the co-deposited CFO preferentially and completely segregates to the pillar sites. To address this, detailed statistical analysis of the AFM measurements for each of the three pitch sizes is shown in Table 7-1. The average pillar height and width above the surface are shown for each of the pitch sizes, which allows for the calculation of the volume of CFO phase held above the surface of the BFO matrix at the template sites for each array. The calculated volume per unit area for all three pitch sizes is shown in Table 7-1, with the values normalized such that the value for the $100 \mathrm{~nm}$ array is equal to 1 . Based on this data, it is shown that the $100 \mathrm{~nm}$ and $150 \mathrm{~nm}$ 
arrays have similar CFO volume per unit area (1 and 1.02, respectively) above the surface. This means that CFO mass is conserved above the surface of the matrix. The pillar area coverage for the $100 \mathrm{~nm}$ and $150 \mathrm{~nm}$ arrays is also similar. Since the pillars are not expected to deviate substantially in diameter during the film growth, this is an indication that the volume of CFO below the surface of the BFO matrix is equal as well. This result agrees with the work of Zheng, et al, which showed that the area coverage of the pillars beneath the matrix surface is equal to the volume fraction of CFO in the adatom flux. ${ }^{71}$ Thus, all CFO flux deposited in the 100 and $150 \mathrm{~nm}$ pitch arrays segregates to the pillars at the template sites.
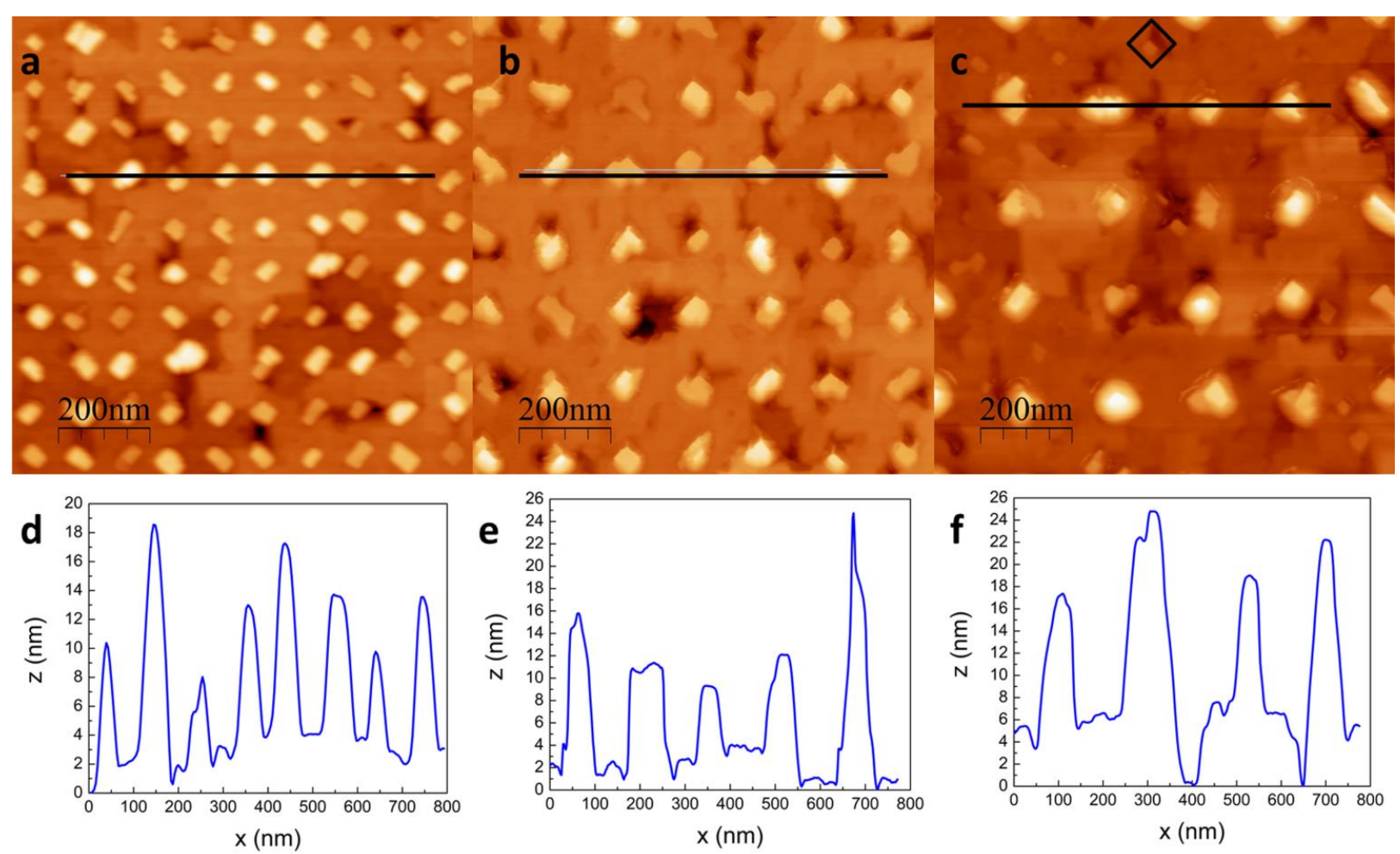

Figure 7-20. (a) Pillar topography with $100 \mathrm{~nm}$ center-to-center distance (pitch); (b) Pillar topography with $150 \mathrm{~nm}$ pitch; (c) Pillar topography with $200 \mathrm{~nm}$ pitch, with defect CFO pillar highlighted; (d-f) Cross section data for: (d) $100 \mathrm{~nm}$, (e) $150 \mathrm{~nm}$, and (f) $200 \mathrm{~nm}$ AFM images along the black lines shown in (a-c). 


\begin{tabular}{|c|c|c|c|c|c|c|}
\hline $\begin{array}{l}\text { Pillar Spacing } \\
\text { (nm) }\end{array}$ & $\begin{array}{l}\text { Mean Pillar Side } \\
\text { Length }(\mathrm{nm})\end{array}$ & $\begin{array}{l}\text { Mean } \\
\text { Height } \\
\text { Matrix } \\
(\mathrm{nm})\end{array}$ & $\begin{array}{l}\text { Pillar } \\
\text { Above } \\
\text { Surface }\end{array}$ & $\begin{array}{l}\text { Fractional } \\
\text { Area Coverage } \\
(\%)\end{array}$ & $\begin{array}{l}\text { CFO } \\
\text { Volume } \\
\text { Surface } \\
\text { Constant } \\
\text { (normalized) }\end{array}$ & $\begin{array}{r}\text { Pillar } \\
\text { Above } \\
\text { in } \\
\text { Area }\end{array}$ \\
\hline 100 & 41 & 6.1 & & 16 & 1.00 & \\
\hline 150 & 55 & 7.6 & & 15 & 1.02 & \\
\hline 200 & 61 & 11.8 & & 9 & 1.10 & \\
\hline
\end{tabular}

Table 7-1. Pillar Dimensions Measured via AFM in Figure 7-20.

For the $200 \mathrm{~nm}$ pitch array, there is excess CFO volume above the surface, as shown by the 1.10 normalized volume per unit area in Table 7-1. A close inspection of Figure 7-20(c) shows that the pillars are more irregularly shaped than in the two arrays with smaller pitch, which indicates that the pillars are most likely not forming the ideal faceted interface with the BFO matrix that is seen in the other arrays. The fractional area coverage of the pillars at the template sites in array is also substantially reduced, meaning that the pillar volume is not sufficient to account for the amount of CFO deposited in the region. The interstitial CFO pillars which form within the BFO matrix in the $200 \mathrm{~nm}$ pitch array account for the remainder of the CFO mass within the array.

A separate work by Zheng et al. estimated the activation energy for surface diffusion of CFO adatoms during PLD growth. ${ }^{71}$ The authors grew a series of unpatterned BFO-CFO nanocomposites at various deposition rates and temperatures using a single 1:1 CFO:BFO composite target. They determined the lateral size of the resulting CFO pillars, $d$. By assuming that the diffusion length was approximately equal to $d$, they showed that the diffusion length was well modeled by assuming:

$d=\sqrt{4 D t}$

where $D$ is the surface diffusivity and $t$ is a characteristic time inversely proportional to the deposition rate. They were able to show that the diffusivity, $D$, had the expected Arrhenius temperature dependence: 
$D=D_{0} e^{-E_{a} / k T}$

where $E_{a}$ is the activation energy for diffusion, $k$ is Boltzmann's constant, $T$ is the growth temperature and $D_{0}$ is a constant that defines the diffusivity in the limit $k T \gg E_{a}$. By fitting the temperature dependence of $d$ they were able to estimate the value of $E_{a}$ as $1.66 \mathrm{eV}$ for CFO adatoms on the surface of the BFO matrix. No uncertainty in $E_{a}$ was published, but based on comments in the text of the article stating that the value is reasonable when compared with that for $\mathrm{CFO}$ adatoms in $\mathrm{CFO}-\mathrm{BaTiO}_{3}(\mathrm{BTO})$ composites $(1.56 \mathrm{eV})$, an uncertainty of $\pm 0.05 \mathrm{eV}$ for $E_{a}$ has been assumed in the calculations. No mention is made of the value for $D$ or $D_{0}$, which is critical for a more precise estimation of the diffusion length.

In order to determine $D_{0}$, the data from the paper was tabulated and fits were performed. ${ }^{2}$ A table showing the pillar diameter, $d$, and the growth rate, $v$, from the paper is shown in Table 1 . In the original work, the authors assumed that the diffusion length was approximately equal to the pillar diameter. However, a more precise estimation is required to quantify $D_{0}$. Since the composition of the films was $1: 1$ BFO:CFO, it is possible to geometrically determine the maximum distance that an adatom must travel if we assume that the pillars are arranged in a close-packed (hexagonal) array. While this assumption is more valid for CFO-BTO composites than it is for CFO-BFO composites, it should still improve the estimate when compared to the assumption presented in the paper. ${ }^{3}$ The maximum capture radius, $r$, for a pillar in a close-packed array was determined geometrically for a 50\% pillar area coverage and found to be:

$$
r=d / \sqrt{3 \sqrt{3}}
$$

The relevant time, $t$, was calculated by assuming that adatoms would only diffuse for a period of time equal to the time required to form one unit cell monolayer of BFO $(\sim 4.1 \AA$ if BFO is strained by the STO substrate $^{4}$ ) in the matrix. The capture radius and monolayer formation time are also shown in Table 7-2. A linear fit was performed to determine the slope of a line: 
where $C$ is an unknown constant that depends on $D_{0}$. From this fit, $D_{0}$ may be extracted using:

$D_{0}=\frac{C^{2}}{4} e^{E_{a} / k T}$

The value of $D_{0}$ was found to be $1.2 \pm 0.8 \times 10^{10} \mathrm{~nm}^{2} / \mathrm{sec}$ after accounting for the uncertainty in both the fit and the assumed uncertainty in $E_{a}$ from the published work.

\begin{tabular}{|l|l|l|l|}
\hline Pillar diameter, $\boldsymbol{d}(\mathbf{n m})$ & Capture radius, $\boldsymbol{r}(\mathbf{n m})$ & $\begin{array}{l}\text { Growth rate, } \boldsymbol{v} \\
(\mathbf{n m} / \mathbf{m i n})\end{array}$ & $\begin{array}{l}\text { Monolayer formation } \\
\text { time, } \boldsymbol{t}(\mathbf{s e c})\end{array}$ \\
\hline 235 & 103 & 0.5 & 49 \\
\hline 170 & 77 & 2 & 12 \\
\hline 125 & 55 & 4 & 6 \\
\hline 100 & 44 & 8 & 3 \\
\hline
\end{tabular}

Table 7-2. Published data for CFO pillar diameter and film growth rate taken from Zheng, et al. ${ }^{71}$ Calculated data for capture radius and monolayer formation time.

With this result, it is possible to calculate the diffusion length of the CFO adatoms in this work. Using Equation (1) and assuming a reasonable uncertainty in substrate temperature of $25 \mathrm{~K}$ based on the nature of our PED heating system, it is found that the diffusion length of the CFO adatoms is $55 \pm 26 \mathrm{~nm}$ at the growth temperature of $615^{\circ} \mathrm{C}$. Since the capture radius for a pillar in an array with $150 \mathrm{~nm}$ pitch is 79 $\mathrm{nm}$, the calculations demonstrate that the diffusion length may be long enough for all adatoms to diffuse to the template sites. However, for the $200 \mathrm{~nm}$ pitch the capture radius of $111 \mathrm{~nm}$ is not in agreement with the uncertainty of the diffusion length.

An analysis of the surface diffusion length for the CFO adatoms is useful to elucidate the kinetics of the growth process. It is clear from the AFM analysis of both the $100 \mathrm{~nm}$ and $150 \mathrm{~nm}$ pitch arrays that all CFO flux is captured at the template sites. However, this is not the case in the $200 \mathrm{~nm}$ pitch array. It 
would appear that the diffusion length of the CFO adatoms is not sufficient for flux that lands far from any of the template sites to move to one of the sites. The maximum diffusion length, $L$, required for adatoms landing in the BFO matrix to reach a template site is given by:

$$
L=\frac{\sqrt{2}}{2} P-r
$$

where $P$ is the array pitch and $r$ is the radius of the pillar. This maximum distance occurs for adatoms that land equidistant from all 4 neighboring template sites at the center of the square formed by the sites. For the $150 \mathrm{~nm}$ pitch with a pillar radius of $27 \mathrm{~nm}, L$ is equal to approximately $79 \mathrm{~nm}$, and for the $200 \mathrm{~nm}$ pitch with a $30 \mathrm{~nm}$ diameter, $L$ is equal to $111 \mathrm{~nm}$. Thus we would expect the diffusion length to fall between 79 and $111 \mathrm{~nm}$ given that defect islands were observed in the $200 \mathrm{~nm}$ pitch array. The calculated diffiusion length of $55 \pm 26 \mathrm{~nm}$ is in reasonable agreement with this geometric result, with the $79 \mathrm{~nm}$ geometric minimum falling within the uncertainty of the calculated diffusion length.

The results of the AFM and simple diffusion analysis above are a good demonstration of the nature of the templating in this work. It has been shown that the CFO islands patterned on the substrate surface act as attachment sites for the epitaxial CFO pillars that form within the BFO matrix and that attachment only occurs at those sites unless there are kinetic limitations - primarily the surface diffusion length. Thus, arbitrary pitch sizes of as much as hundreds of nanometers may be possible by tailoring the growth conditions, such as the growth temperature and the deposition rate, to the template pattern. In addition, smaller pitch sizes should be readily achievable by refining the EBL and ion etching processes to improve resolution.

\section{Magnetic Properties of Templated Nanocomposites}

It is worthwhile to characterize the magnetic and ferroelectric properties of the composite as a means of comparing the template sample with other unpatterned composites in the literature. Conventional measurements to determine the magnetic anisotropy in the sample through the use of a vibrating sample 
magnetometer (VSM) or superconducting quantum interference device (SQUID) would be fruitless, however, as the patterned arrays cover only about $0.01 \%$ of the overall surface and any signal would be masked by the pillars that form spontaneously outside of the patterned arrays. Thus, magnetic force microscopy (MFM) is the only viable approach to study the anisotropy characteristics of the pillars.

The sample was demagnetized by an applied in-plane damped oscillatory magnetic field in order to drive the pillars to the minimum energy magnetization state. Figure 7-21 shows MFM scans of the 150 $\mathrm{nm}$ pitch array after the demagnetization process, with a three-dimensional rendering of the topography shown and the magnetic phase overlaid as the color in the image. In Figure 7-21(c), red and blue represent the positive and negative out-of-plane magnetizations (arbitrary sign), while green represents regions of small magnetic response. The nature of the magnetostatic interactions between neighboring pillars with in-plane magnetization makes the interpretation of the MFM images complicated. If the pillars are magnetized in-plane, the MFM phase contrast should be positive on one side of the pillar and negative on the other, with a neutral region in the center of the pillar due to the nature of the magnetic dipole fringe field that curls over the surface. For pillars with out of plane magnetization, the phase should be uniform across the surface of the pillar. A schematic of the two configurations is shown in Figure 7-21(a).
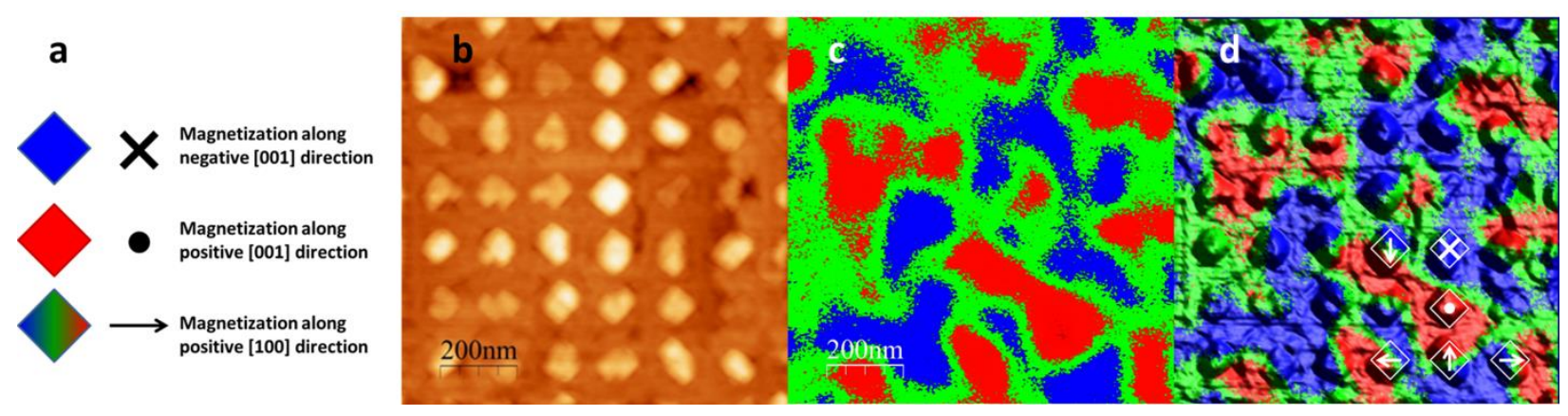

Figure 7-21. Magnetic force microscopy analysis of $150 \mathrm{~nm}$ pitch array. a) Schematic of possible pillar magnetization contrasts; b) Topography image; c) MFM phase image; d) 3-dimensional representation of pillar topography with phase overlaid as color. Arrows in (d) represenent the observed magnetization directions for selected pillars. 
Figure 7-21(d) shows the MFM phase contrast overlaid on a three-dimensional rendering of the surface topography. This technique is useful to show which pillars have in-plane and out-of-plane magnetization. Examples of magnetization along all six possible directions are highlighted in the figure. There is no clear preference for in-plane or out-of-plane magnetization, indicating that uniaxial anisotropy in the pillars is minimal and that the magnetocrystalline anisotropy in CFO along the three [100] axes dominates the magnetic behavior. This result, while different from many reports in the literature, agrees with other composite films grown by PED, as discussed in the previous chapter. ${ }^{181}$

\section{Ferroelectric Properties of Templated Nanocomposites}

To characterize the ferroelectric properties of the sample, piezoresponse force microscopy (PFM) was performed. PFM results topography, amplitude and phase scans are shown in Figure 7-22. The amplitude scan (Figure 7-22(b)) shows that there is no ferroelectric response from the CFO pillars, indicated by the black coloring at pillar sites. The amplitude scan also shows that there is a high density of $180^{\circ}$ domain walls in the BFO matrix, as indicated by the dark lines throughout the pattern where the ferroelectric response vanishes. ${ }^{147}$ The phase image shows clear domains which are $180^{\circ}$ out-of-phase, in agreement with the amplitude scan. The domain structure of the composite is consistent with results shown elsewhere, with small $180^{\circ}$ reverse domains forming near the interface with the pillars. ${ }^{182}$ The presence of reversal domains can most likely be attributed to point defects in the BFO due to the presence of the pillars. In uniform BFO films, reversal domains along the out-of-plane direction are not generally observed. $^{178}$ 


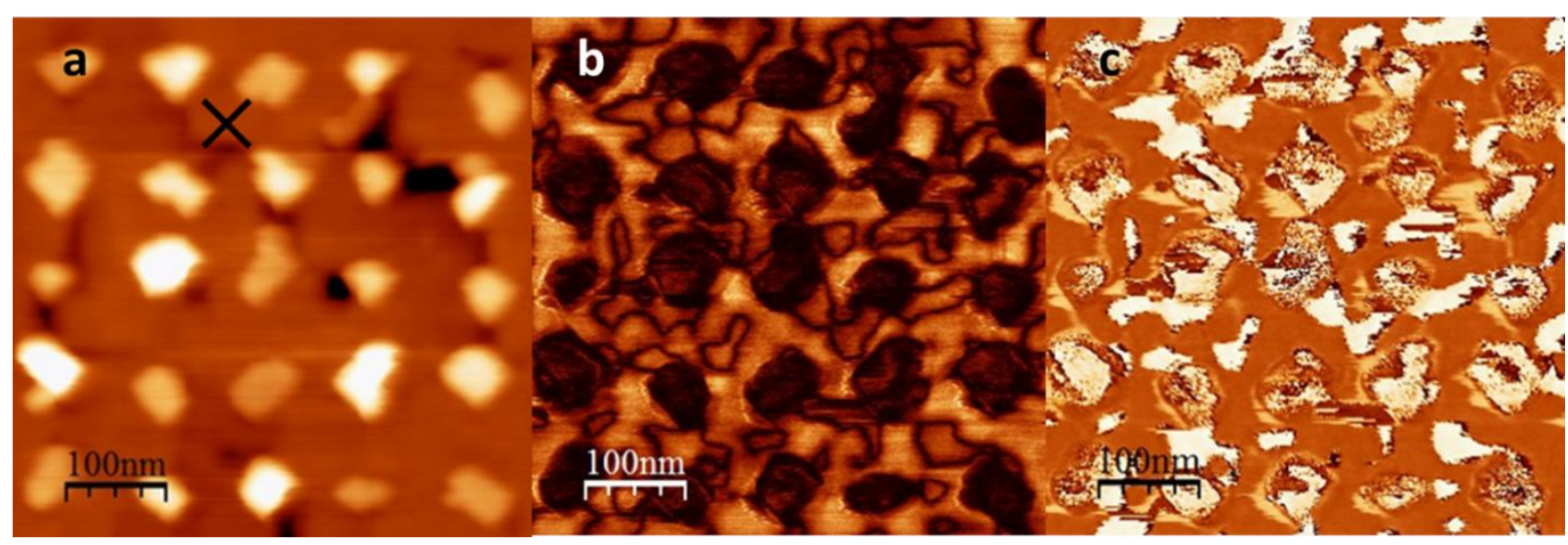

Figure 7-22. (a) Contact mode topography image taken during piezoresponse force microscopy (PFM) measurement (' $X$ ' indicates the site of switching spectroscopy-PFM (SS-PFM) measurement in Fig. 6); (b) Ferroelectric amplitude image corresponding to (a) (dark indicates low PFM response); (c) Ferroelectric phase image corresponding to (a) (white and orange correspond to oppositely oriented domains along the outof-plane direction).

Localized ferroelectric hysteresis measurements were also performed using switching spectroscopyPFM (SS-PFM) within the matrix region shown in Figure 7-23. ${ }^{148}$ The location of the measurement is marked with an ' $X$ ' in Figure 7-22(a). An Asylum Research ASYELEC-01 Ir coated tip was used for the measurements, with the conductive $\mathrm{Nb}$-doped $\mathrm{SrTiO}_{3}$ serving as a bottom electrode. The tip has a free air resonant frequency of $86.1 \mathrm{kHz}$ and a contact resonant frequency, $f$, of $310.9 \mathrm{kHz}$ and a quality factor, $Q$, of 132. Switching-spectroscopy PFM (SS-PFM) hysteresis measurements were performed using the tip to switch small domains in the matrix. The voltage was scanned three times over a range of $+8 \mathrm{~V}$ to $-8 \mathrm{~V}$ applied tip bias and the ferroelectric response was measured each time at $0 \mathrm{~V}$ applied bias, with an alternating current drive amplitude, $V_{a c}$, of $500 \mathrm{mV}$. This technique is standard for SS-PFM measurements. ${ }^{9}$ The resulting PFM amplitude and out-of-plane phase loops were then averaged to produce the loops shown in Figure 7-23.

The hysteresis loops are shown in Figure 7-23. Figure 7-23(a) shows the out-of-plane phase, with clear switching at applied voltages of approximately $2.5 \mathrm{~V}$. The measured tip displacement amplitude is shown in Figure 7-23(b), with the traditional butterfly curve shape. Estimates of the value of the 
piezoelectric $d_{33}$ coefficient are difficult due to the complex nature of the tip-sample interactions, but estimations can be made using the measured tip displacement shown in Figure 7-23(b). ${ }^{183}$

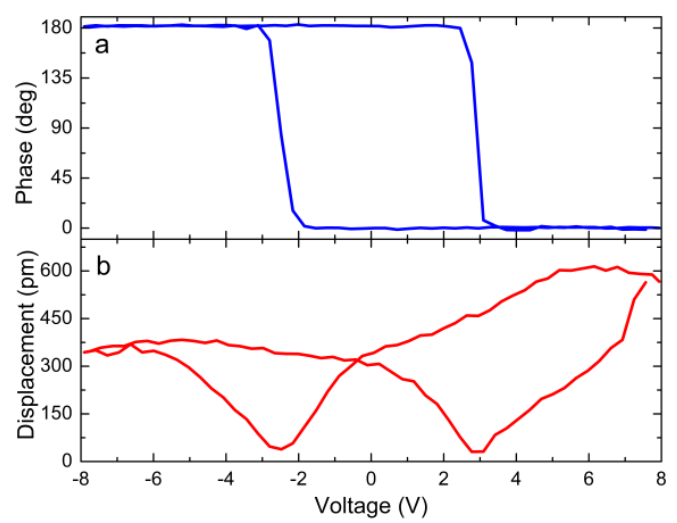

Figure 7-23. (a) Out-of-plane ferroelectric phase loop in $\mathrm{BiFeO3}$ (BFO) matrix measured using piezoresponse force microscopy (PFM); (b) Measured tip displacement showing the expected butterfly loop behavior.

Separate PFM measurements were also performed on the nanocomposite sample fabricated for electron microscopy analysis. This sample was discussed in Section 2 of this chapter. For this sample, both out-of-plane and lateral PFM analysis were performed using the "Vector PFM" option on the Asylum Cypher system. ${ }^{183}$ The results of this measurement are shown in Figure 7-24. Unlike the PFM results for the previous sample, the out-of-plane domain structure is uniform, which is commonly seen in uniform BFO films grown on STO (001) substrates. ${ }^{178}$ For the in-plane PFM phase images, shown in Figure 7-24(E), there is clear domain wall pinning at the pillar sites, in agreement with what was observed for the unpatterned nanocomposites in Chapter 6. The effect of the square grid of pillars is to make rectangular domain structures, as domain walls pin between neighboring pillars. This result serves as further proof of the argument presented in Chapter 6 regarding the pinning of domain walls at pillar sites. 


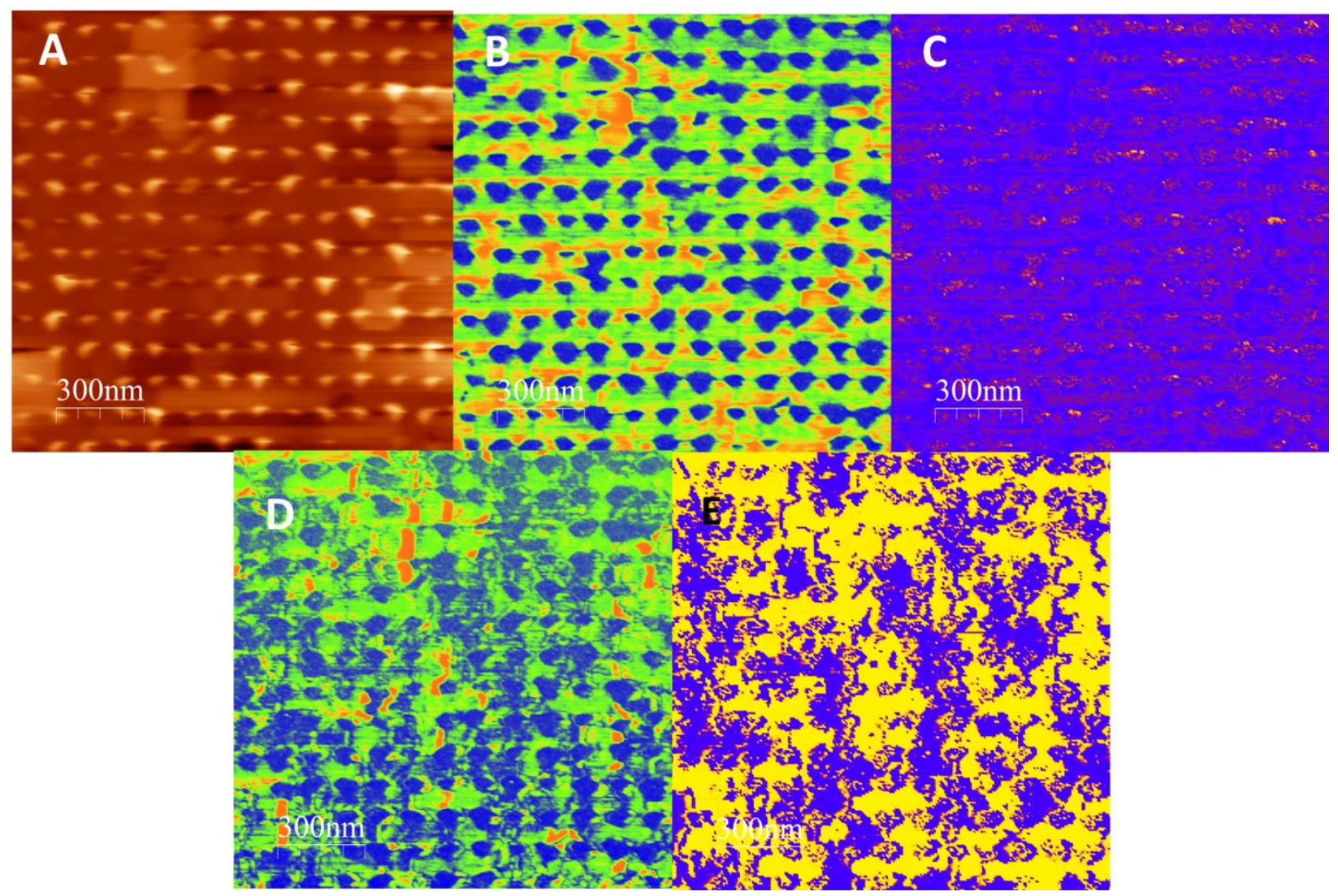

Figure 7-24. Vector piezoresponse force microscopy measurement of templated sample. A) Topography; B) Vertical amplitude response; C) Vertical phase response; D) Lateral amplitude response; E) Lateral phase response.

\section{Comparison with Unpatterned Nanocomposites}

As a means of considering further work and possible applications of these nanocomposites, it is worthwhile to examine the similarities and differences between the patterned nanocomposites presented in this chapter and the unpatterned nanocomposites presented in Chapter 6. In terms of similarities, the template composites share the majority of the same structural and functional properties as the spontaneously formed nanocomposites. This work has shown that an epitaxial island of CFO can serve as a good template for an epitaxial CFO nanopillar in an epitaxial BFO matrix, with no obvious changes in the crystal quality of the matrix and pillar away from the template interface. Through magnetic force microscopy and piezoresponse force microscopy, it has been shown that the nanocomposite exhibits the same multiferroic properties as the spontaneously formed nanocomposites. The magnetic anisotropy of 
the pillars is different from those grown by other groups in the literature, but is in agreement with what has been observed in our group when the composites are grown via PED. ${ }^{181}$ The ferroelectric domain structure, both in-plane and out-of-plane, has been characterized via PFM and shows excellent agreement with the results presented in Chapter 6.

The differences between the patterned and unpatterned nanocomposites are primarily observed through high-resolution TEM analysis. The nature of the interface between the pillar and island template is more complex than in the case of the spontaneously formed pillars. It is likely that there are dislocations present within the CFO pillar that are not observed in the spontaneously formed pillars. This could be attributed to the grain structure of the CFO island after patterning. The EBL island pattern is likely to overlap multiple CFO epitaxial grains prior to etching. It is unclear how the interface between these grains would behave during the growth process. However, more complex Moire fringing patterns and interfacial images are observed via TEM in the patterned composites than in those that form spontaneously. Further examination of these properties would be enlightening in future work.

\section{Applications}

\section{Reconfigurable Array of Magnetic Automata}

One of the primary motivations for this work was the possible application of patterned epitaxial CFOBFO nanocomposites for use in the RAMA magnetic logic architecture. ${ }^{3}$ Significant progress has been made in this regard. This work has demonstrated the ability to pattern the nanocomposites to small enough pitch sizes so that it may be possible to produce dipole coupling between neighboring pillars, according to computational modeling work performed by our collaborators. ${ }^{28}$ The demonstration of such coupling was not possible in the nanocomposites produced in this work due to the lack of perpendicular anisotropy in the CFO nanopillars. However, the technique developed should be applicable to the growth of CFO-BFO nanocomposites, along with other spinel-perovskite nanocomposites, using a variety of 
different growth techniques. Future growth of these composites via pulsed laser deposition or sputtering using substrates with island templates would be worthwhile.

The other key aspect of RAMA is the ability to reconfigure the magnetic logic circuit through the patterning of nanoscale domains in the BFO matrix. Such experiments were possible in the samples fabricated in this thesis. In the sample used for electron microscopy analysis, ferroelectric domains in the shape of an inverter were patterned in the BFO matrix via PFM. The results of this experiment are presented in Figure 7-25. The sample was poled at a bias of $-9 \mathrm{~V}$ using the PFM tip over a region that would match the desired pattern of a logical inverter in the RAMA architecture. A map of this pattern is shown in Figure 7-25(A). A second out-of-plane PFM measurement was then made to examine the domain structure after poling. The amplitude and phase images of this measurement are shown in Figure 7-25(C-D). During the measurement the tuning of the PFM tip drifted, resulting in the phase signal crossing the border between $270^{\circ}$ and $-90^{\circ}$. These two angles are equivalent physically, but are measured differently by the microscope. To correct for this, a phase map that equates the two angles was used. It is clear from the phase image that the poled region is approximately $180^{\circ}$ out-of-phase with the unpoled region, indicating a reversal of the ferroelectric polarization. While the fidelity to the original pattern is not perfect, it is clear that it is possible to pattern the domain structure on this scale for use in an architecture such as RAMA. 


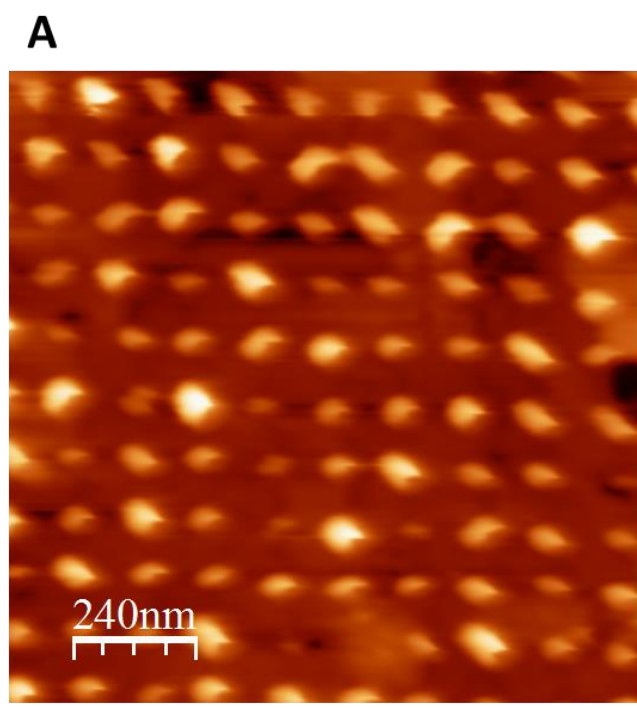

C

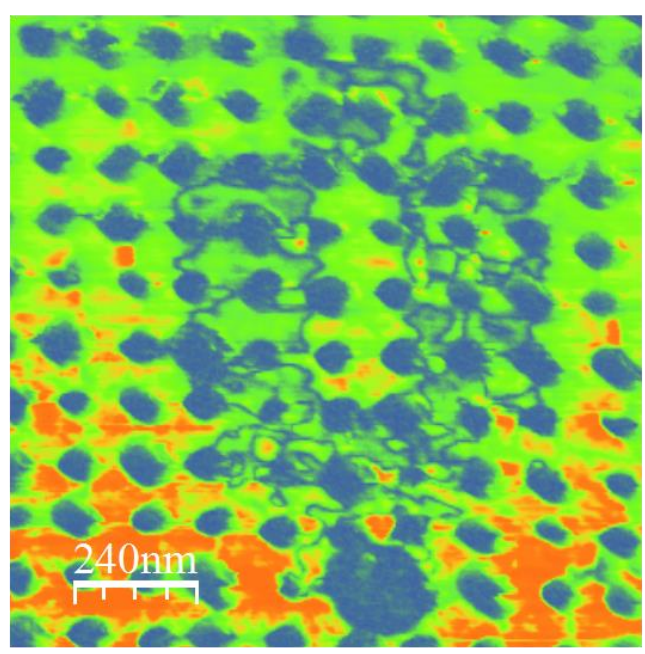

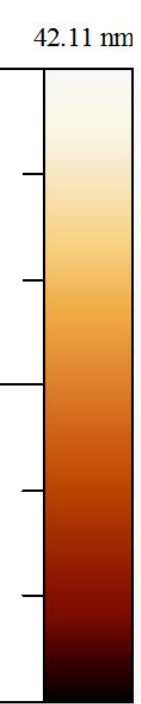

$0.00 \mathrm{~nm}$ $3.00 \AA$

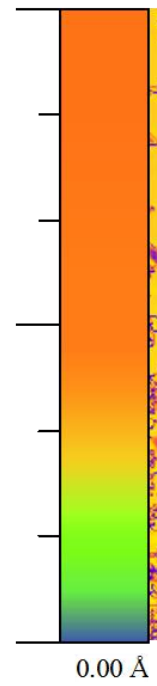

B

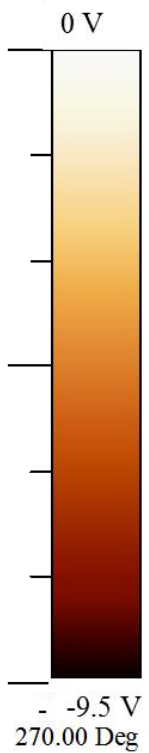

D

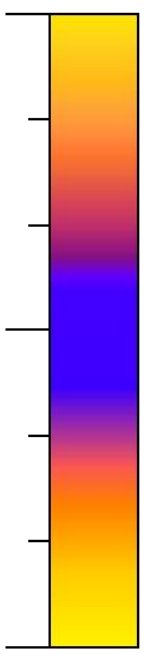

-90.00 Deg

Figure 7-25. Lithographic patterning of BFO domain structure via piezoresponse force microscopy. A) Topography of nanopillar array; B) Applied bias map; C) PFM amplitude image following poling; D) PFM phase image after poling.

\section{X-Ray Nanodiffraction}

To examine the strain induced by poling the ferroelectric matrix, scanning x-ray nanodiffraction was performed at the Nanoprobe Beamline at the Advanced Photon Source at Argonne National Lab. The proposed experiments were based on previous work done on the beamline, which indicated that a strain of approximately $0.1 \%$ is induced in $\mathrm{Pb}(\mathrm{Zr}, \mathrm{Ti}) \mathrm{O}_{3}$ films when they are poled via $\mathrm{PFM}^{77}$ The patterned nanocomposites offer a unique opportunity in this regard, as a periodic grid of pillars would allow for 
easier interpretation of the results during nanodiffraction. The nanocomposite also is an ideal candidate for Bragg x-ray ptychography, a technique recently developed by scientists at Argonne to increase the resolution of nanodiffraction measurements through the use of Fourier analysis of coherently scattered diffraction patterns from nanoscale features. ${ }^{184}$ The epitaxial interface between the CFO pillar and the BFO matrix with $\{110\}$-type facets could be expected to produce coherent diffraction of incident x-rays near the Bragg peak. This scattering would be analogous to the Kiessig fringes observed at the epitaxial interface between a film and substrate. Both experiments were pursued during a week of beam time at the Nanoprobe Beamline using the sample presented in Section 3 of this chapter for analysis of the growth kinetics.

Two intriguing preliminary results are presented below in Figure 7-26. In Figure 7-26(A), a map of the centroid of the BFO $(002)_{\mathrm{pc}}$ peak is shown. This image was taken during an alignment scan to find the patterned arrays. The centroid represents the position of the diffraction peak, analogous to the value of $2 \theta$ in a diffraction measurement performed via conventional techniques. It is clear that the area of the array has a different peak position than the unpatterned regions, indicating that some strain is induced by the underlying template. A representative image of the $\mathrm{BFO}$ peak obtained on the CCD camera is shown in Figure 7-26(B). This image show the primary BFO peak, along with what is believed to be a coherently scattered peak to the right of the primary peak. This peak would likely be the result of the interface between the CFO pillar and BFO matrix. Further analysis of the results was not possible due to low signal intensity and no evidence of the patterned ferroelectric domains in the matrix. Continued improvement of the algorithms to perform ptychography analysis would also be beneficial, as the model is not yet equipped to handle the presence of two distinct crystal phases in the same plane. It was concluded that a thicker and more highly crystalline sample would be needed to achieve the desired results. Later growths, such as the one presented in the electron microscopy section (Section 2) of this chapter achieved better crystallinity, but difficulties with the PED prevented a second set of experiments at the beamline. Future experiments continuing this line of research would be intriguing. 

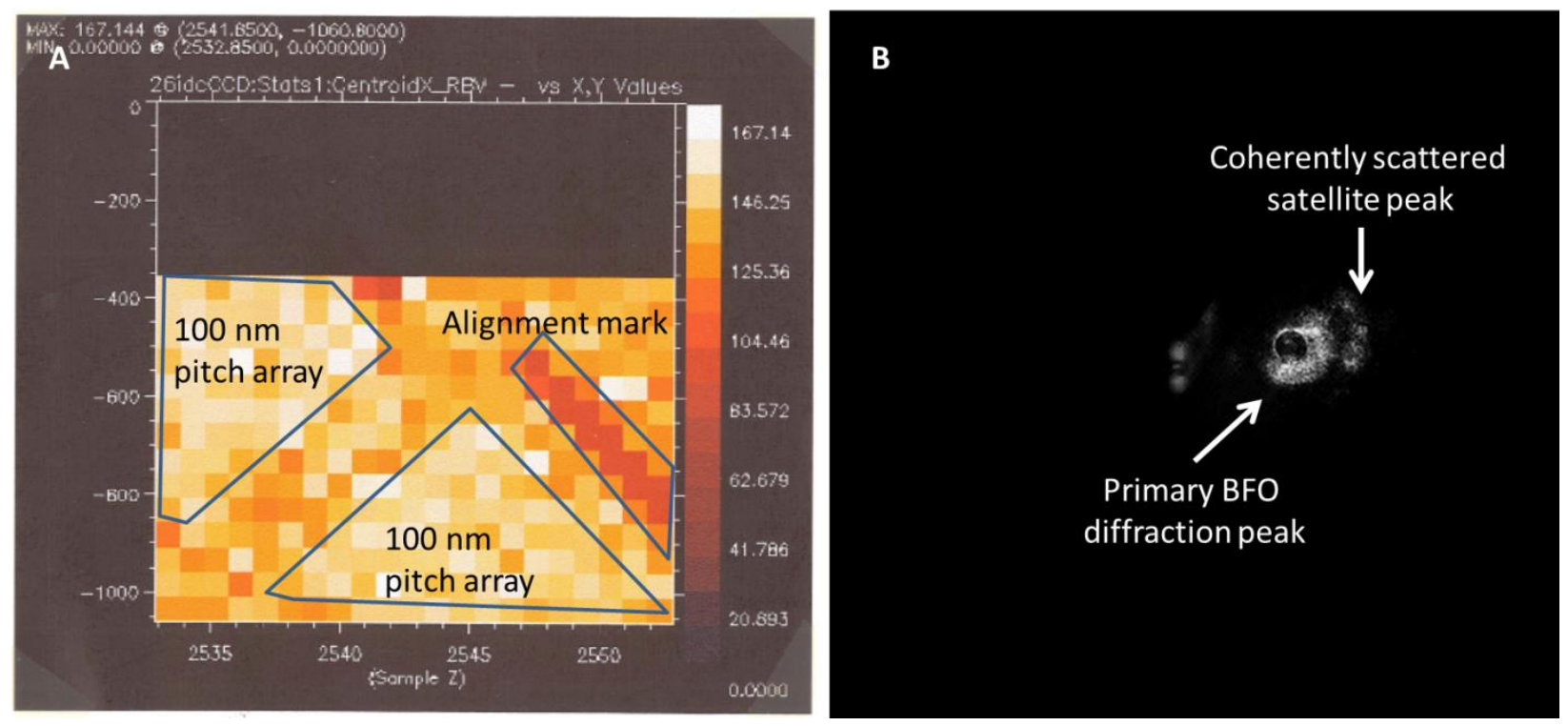

Figure 7-26. A) Nanoscale map of the centroid of the BFO diffraction peak, showing that the lattice parameter of the BFO film is different in the area of the patterned array than in the unpatterned regions; $B$ ) CCD image of diffracted peak at the BFO (002) $)_{\text {pc }}$ Bragg condition, showing coherent scattering that may be due to the presence of a pillar.

\section{Summary}

As a means of patterning the growth of the epitaxial $\mathrm{CoFe}_{2} \mathrm{O}_{4}$ pillars in a $\mathrm{CoFe}_{2} \mathrm{O}_{4}(\mathrm{CFO})-\mathrm{BiFeO}_{3}$ (BFO) nanocomposite, electron-beam lithography has been employed to produce a template substrate with CFO islands on the surface. This template has been used to direct the self-assembly of CFO-BFO nanocomposites grown via pulsed electron deposition. Structural and chemical characterization of these samples via transmission electron microscopy verified that the CFO island chemically promotes the nucleation of the pillars. The analysis showed that the pillars exhibit similar crystallinity and strain conditions to the spontaneously formed pillars that were examined in the previous chapter.

Characterization of these samples via magnetic force microscopy and piezoresponse force microscopy showed that the multiferroic properties of the patterned samples match those of the spontaneously formed nanocomposites. By demonstrating the ability to pattern these nanocomposites via directed self-assembly, this work has laid the materials foundation for future technologies employing spinel-perovskite 
nanocomposites, such as the Reconfigurable Array of Magnetic Automata, and a variety of experiments that would be more difficult on unpatterned composites. 


\section{Summary and Future Work}

This work has focused on the growth of epitaxial complex oxide films and nanocomposites via pulsed electron deposition (PED). I have presented results discussing the microstructures and properties of uniform films and nanocomposites grown via this technique and contrasted these results with those observed in films grown via pulsed laser deposition (PLD). The directed self-assembly of epitaxial nanocomposites through templating has also been demonstrated, representing the first such demonstration of patterning in complex oxide nanocomposites. This chapter summarizes these results and suggests interesting avenues for continued work.

\section{Summary}

\section{Growth of Complex Oxide Films via Pulsed Electron Deposition}

Pulsed electron deposition (PED) remains a film growth technique in its infancy when compared to the more established PLD approach. In this work, I have demonstrated the growth of epitaxial $\mathrm{BiFeO}_{3}$ (BFO), $\mathrm{La}_{0.72} \mathrm{Sr}_{0.28} \mathrm{MnO}_{3}$ (LSMO) and $\mathrm{CoFe}_{2} \mathrm{O}_{4}$ (CFO) via $\mathrm{PED}$ on various substrates. The structural properties of these materials have been characterized via atomic force microscopy (AFM) and x-ray diffraction (XRD). Layer-by-layer growth of all three materials has been observed with the appropriate substrate selection. BFO films grown on $\mathrm{SrTiO}_{3}(\mathrm{STO})$ and $\mathrm{LaAlO}_{3}(\mathrm{LAO})$ exhibit layer-by-layer growth, with those grown on LAO demonstrating the expected rhombohedral-to-tetragonal phase transition due to compressive epitaxial strain. CFO films exhibit Volmer-Weber island growth on STO, while demonstrating layer-by-layer growth on $\mathrm{MgO}$ due to an excellent lattice match. The dependence of the magnetic anisotropy of CFO films grown on $\mathrm{MgO}$ on the film thickness has also been explained through analysis of the strain relaxation and magnetic domain wall motion. Finally, LSMO films grown on STO exhibit smooth layer-by-layer growth, while films grown on LAO demonstrate the intermediate StranskiKrastanov layer-then-island growth mode. These results are the first demonstration of the various epitaxial growth modes for films grown via PED and represent a significant improvement over previous 
work in the literature. Recent works by other groups have also demonstrated similar results, ${ }^{170}$ offering encouragement that the technique will continue to improve in future years.

\section{Spontaneously-formed $\mathrm{CoFe}_{2} \mathrm{O}_{4}-\mathrm{BiFeO}_{3}$ Epitaxial Nanocomposites}

To benchmark the growth of CFO-BFO nanocomposites via PED, unpatterned composites were grown and characterized to determine their magnetic and ferroelectric properties. Nanocomposite films with excellent crystal quality were obtained, demonstrating the first example of Kiessig fringes in a nanocomposite. The PED growth technique was found to produce different strain conditions in the CFO nanopillars than in films demonstrated in the literature. This result is believed to be a result of the difference in growth rates achieved in PED when compared to PLD, which allows for full relaxation of out-of-plane residual strain. In-plane compressive strain is observed in the thinnest films, which is believed to result from the lattice mismatch with both the STO substrate and BFO matrix along the inplane directions. For thicker films, complete strain relaxation is found, which is analogous to the relaxation of strain in epitaxial films through the formation of misfit dislocations. Due to the different strain states, unexpected in-plane magnetic anisotropy was observed for thin films and was explained through analysis of the elastic and shape anisotropy contributions to the overall magnetic anisotropy. Analysis of the ferroelectric properties of the BFO matrix via piezoresponse force microscopy showed that in-plane domain walls preferentially pin at the interface of the matrix with the CFO pillar, offering a potential pathway to control the in-plane domain structure.

\section{Directed Self-Assembly of $\mathrm{CoFe}_{2} \mathrm{O}_{4}-\mathrm{BiFeO}_{3} \mathrm{Nanocomposites}$}

To enable future device applications for CFO-BFO and other spinel-perovskite nanocomposites, a large portion of this work was dedicated to developing a means to direct the self-assembly of these nanocomposites. In this thesis, I have demonstrated that by patterning CFO islands on the surface of a STO substrate, it is possible to control the growth site of the pillars that form in the nanocomposite. Structural characterization via transmission electron microscopy (TEM) has shown that the CFO islands act to chemically promote the nucleation of the nanopillars. The growth kinetics has also been explained 
through the use of atomic force microscopy characterization of pillar arrays with different pitch spacings. It was found that the template islands absorb all deposited CFO flux if the diffusion length of the adatoms is sufficiently large to allow for diffusion of all adatoms to the sites. Characterization of the structural

properties via TEM and x-ray diffraction has verified that the materials have comparable crystal quality to those that form spontaneously. Analysis by magnetic and piezoresponse force microscopy has verified that the patterned nanocomposites exhibit the same multiferroic properties as the unpatterned samples examined in Chapter 6. The work has also shown that it is possible to pin the in-plane ferroelectric domain walls at the patterned pillar sites. By patterning more complex pillar geometries, it may be possible to produce unique functional domain structures using this approach.

\section{Future Work}

\section{Growth of Complex Oxide Films via Pulsed Electron Deposition}

As a relatively new technology with complex growth dynamics, the capabilities of PED are still not fully understood. However, the ability to grow high quality epitaxial films with controllable morphology is important if the technique is to be competitive with the more developed PLD and MBE approaches. To that end, it would be useful to integrate a RHEED in situ monitoring system to fully realize the capabilities of “electron MBE." Such a RHEED system would be useful to monitor film thickness and characterize epitaxial modes during growth. This would make the PED an ideal tool for multilayer, superlattice and codeposited alloy growth given the multiple electron guns present in the system.

Other approaches to improve film quality could also be pursued. There are two primary constraints that must be considered to produce uniform films that are particulate free and exhibit smooth surfaces. First, a sufficiently large background gas pressure and long distance from target to substrate is needed to thermalize the adatoms prior to deposition on the substrate. Second, a sufficiently low electron pulse energy must be used to reduce particulate emission. Both of these factors lead to reduced deposition rates, making growth of even relatively thin films a slow process. To overcome this, future work might focus on 
the use of off-axis deposition, where the substrate is no longer in the path of the plume. This approach has been employed in PLD to produce high quality films. ${ }^{185}$ By moving the substrate off-axis, emitted atoms must be scattered by the gas in the chamber, ensuring that they are thermalized prior to deposition. In PLD, this approach leads to a lower deposition, but in the case of PED, it may actually be possible to increase the deposition rate with this approach. By moving the substrate off-axis, concerns over particulate emission and adatom thermalization would be reduced, which might allow future experimenters to use a higher pulse energy and shorter distances from target to sample.

\section{Ferroelectric Domain Wall Pinning in $\mathrm{CoFe}_{2} \mathrm{O}_{4}-\mathrm{BiFeO}_{3} \mathrm{Nanocomposites}$}

The results presented here on the investigation of in-plane domain wall pinning represent only an initial result of what should be a more detailed study of the phenomenon. Understanding the origin of the pinning process, which were suggested here to be dislocations at the CFO-BFO vertical interface or oxygen vacancies, could allow for more detailed engineering of the domain structures. The ability to pattern the nanocomposites offers a unique opportunity to study the domain structure in these nanocomposites. By templating the pillar sites with various pitches, grid orientations and pillar configurations (i.e. non-square grids), a wide variety of domain geometries may be achievable. The results presented here have shown that it should be possible to pattern a wide variety of pillar geometries

on the same substrate, ${ }^{100}$ making these experiments easy to perform. Perhaps the first step in the process would be to rotate the pillar grid $45^{\circ}$ so that the nearest neighbor pillars are along the $\langle 110\rangle$ directions, which is the axis where in-plane stripe domains have been observed in uniform BFO films. ${ }^{177}$

\section{Directed Self-Assembly of Nanocomposites}

Further analysis of the directed self-assembly of the nanocomposites from both a materials science and a device perspective should prove very rewarding. While there are clear applications of the CFO-BFO nanocomposites for the Reconfigurable Array of Magnetic Automata, ${ }^{3}$ plenty of other applications for matrix-pillar nanocomposites also exist. Others have already adapted the work presented here by demonstrating that the patterning of Fe metallic islands can promote the self-assembled growth of Fe- 
$\mathrm{LaSrFeO}_{4}$ nanocomposites. ${ }^{101,186}$ Numerous other examples of spinel-perovskite nanocomposites were discussed in Chapter 2, demonstrating several different novel properties. The technique used here should be readily applicable to spinel-perovskite nanocomposites formed from a variety of different materials. Perovskite materials also have applications for solar cells, ${ }^{187}$ catalysis, ${ }^{188}$ and thermoelectrics. ${ }^{189}$ It is easy to envision device applications for each system where periodically embedded pillars could prove useful as electrodes or for other purposes.

Continuing research should focus on reducing the spacing between the island sites, which would involve careful process engineering to optimize the electron-beam lithography (EBL) and reactive ion etching processes. An alternative to EBL might include the use of polymeric self-assembly to produce smaller pitch sizes that cover a larger area of the substrate. ${ }^{190}$ It would also be of interest to examine the effect that the initial CFO morphology and microstructure has on the subsequent pillar structure. Others have demonstrated the ability to pattern small epitaxial single-crystal islands of CFO via a combined EBL and sol-gel process. ${ }^{191,192}$ This approach might be useful as an alternative to the top-down etching process if a single crystal seeding island is desired.

The ability to pattern the growth of these nanocomposites also enables a wide variety of experiments that may not have been possible with unpatterned samples. Preliminary x-ray nanodiffraction experiments were presented in Chapter 7, but follow-up experiments were not possible in this work. The ability to produce regular arrays of pillars makes the nanodiffraction and ptychography experiments presented there far more practical, but other measurements might also prove fruitful. Scientists at Argonne National Lab suggested ${ }^{193}$ that experiments leveraging the periodic spacing of the pillars to perform grazing incidence diffraction measurements would improve the signal and produce intriguing results. ${ }^{194}$ Time-resolved synchrotron measurements measuring the transient strain induced in the pillars during the switching process would also be of interest. ${ }^{195}$ 


\section{References}

1. Eerenstein, W., Mathur, N. D. \& Scott, J. F. Multiferroic and magnetoelectric materials. Nature 442, 759-765 (2006).

2. Ramesh, R. \& Spaldin, N. A. Multiferroics: progress and prospects in thin films. Nat Mater 6, 21-29 (2007).

3. Wolf, S. A., Jiwei Lu, Stan, M. R., Chen, E. \& Treger, D. M. The Promise of Nanomagnetics and Spintronics for Future Logic and Universal Memory. Proceedings of the IEEE 98, 2155-2168 (2010).

4. Wolf, S. A., Chtchelkanova, A. Y. \& Treger, D. M. Spintronics-A retrospective and perspective. IBM Journal of Research and Development 50, 101-110 (2006).

5. Wolf, S. A. et al. Spintronics: A Spin-Based Electronics Vision for the Future. Science 294, 1488 1495 (2001).

6. Fiebig, M. Revival of the magnetoelectric effect. J. Phys. D: Appl. Phys. 38, R123-R152 (2005).

7. Yan, L. et al. Review of magnetoelectric perovskite-spinel self-assembled nano-composite thin films. J Mater Sci 44, 5080-5094 (2009).

8. Fiebig, M., Lottermoser, T., Lonkai, T., Goltsev, A. V. \& Pisarev, R. V. Magnetoelectric effects in multiferroic manganites. Journal of Magnetism and Magnetic Materials 290-291, 883-890 (2005).

9. Lee, S. et al. Giant magneto-elastic coupling in multiferroic hexagonal manganites. Nature 451, 805808 (2008).

10. Lee, J. H. et al. A strong ferroelectric ferromagnet created by means of spin-lattice coupling. Nature 466, 954-958 (2010).

11. Calderón, M. J. et al. Magnetoelectric coupling at the interface of $\mathrm{BiFeO}_{3} / \mathrm{La}_{.7} \mathrm{Sr}_{3} \mathrm{MnO}_{3}$ multilayers. Phys. Rev. B 84, 024422 (2011).

12. Kim, H.-S. et al. Self-Assembled Single-Phase Perovskite Nanocomposite Thin Films. Nano Letters 10, 597-602 (2010).

13. Zheng, H. et al. Multiferroic $\mathrm{BaTiO}_{3}-\mathrm{CoFe}_{2} \mathrm{O}_{4}$ Nanostructures. Science 303, 661-663 (2004).

14. Zavaliche, F. et al. Electric Field-Induced Magnetization Switching in Epitaxial Columnar Nanostructures. Nano Letters 5, 1793-1796 (2005).

15. Parkin, S. S. P. et al. Giant tunnelling magnetoresistance at room temperature with $\mathrm{MgO}(100)$ tunnel barriers. Nature Materials 3, 862-867 (2004).

16. Baibich, M. N. et al. Giant Magnetoresistance of (001)Fe/(001)Cr Magnetic Superlattices. Phys. Rev. Lett. 61, 2472-2475 (1988).

17. Katine, J. A., Albert, F. J., Buhrman, R. A., Myers, E. B. \& Ralph, D. C. Current-Driven Magnetization Reversal and Spin-Wave Excitations in Co /Cu /Co Pillars. Phys. Rev. Lett. 84, 3149 3152 (2000).

18. Urushibara, A. et al. Insulator-metal transition and giant magnetoresistance in $\mathrm{La}_{1-\mathrm{x}} \mathrm{Sr}_{\mathrm{x}} \mathrm{MnO}_{3}$. Phys. Rev. B 51, 14103-14109 (1995).

19. Noh, J. S. et al. Magnetotransport in manganite trilayer junctions grown by $90^{\circ}$ off-axis sputtering. Applied Physics Letters 79, 233-235 (2001).

20. O’Donnell, J., Andrus, A. E., Oh, S., Colla, E. V. \& Eckstein, J. N. Colossal magnetoresistance magnetic tunnel junctions grown by molecular-beam epitaxy. Applied Physics Letters 76, 1914-1916 (2000).

21. Sefrioui, Z. et al. Tunnel magnetoresistance in $\mathrm{La}_{0.7} \mathrm{Ca}_{0.3} \mathrm{MnO}_{3} / \mathrm{PrBa}_{2} \mathrm{Cu}_{3} \mathrm{O}_{7} / \mathrm{La}_{0.7} \mathrm{Ca}_{0.3} \mathrm{MnO}_{3}$. Applied Physics Letters 88, 022512-022512-3 (2006).

22. Cowburn, R. P. \& Welland, M. E. Room Temperature Magnetic Quantum Cellular Automata. Science 287, 1466-1468 (2000).

23. Allwood, D. A. et al. Submicrometer Ferromagnetic NOT Gate and Shift Register. Science 296, 2003-2006 (2002).

24. Imre, A. et al. Majority Logic Gate for Magnetic Quantum-Dot Cellular Automata. Science 311, 205 -208 (2006). 
25. Csaba, G., Lugli, P. \& Porod, W. Power dissipation in nanomagnetic logic devices. in 4th IEEE Conference on Nanotechnology, 2004346 - 348 (2004). doi:10.1109/NANO.2004.1392346

26. Atulasimha, J. \& Bandyopadhyay, S. Bennett clocking of nanomagnetic logic using multiferroic single-domain nanomagnets. Applied Physics Letters 97, 173105-173105-3 (2010).

27. D’Souza, N., Atulasimha, J. \& Bandyopadhyay, S. Four-state nanomagnetic logic using multiferroics. Journal of Physics D: Applied Physics 44, 265001 (2011).

28. Kabir, M., Stan, M. R., Wolf, S. A., Comes, R. B. \& Lu, J. RAMA: a self-assembled multiferroic magnetic QCA for low power systems. in Proceedings of the 21st edition of the great lakes symposium on Great lakes symposium on VLSI 25-30 (ACM, 2011). doi:10.1145/1973009.1973015

29. Frank, F. C. \& Merwe, J. H. van der. One-Dimensional Dislocations. I. Static Theory. Proc. R. Soc. Lond. A 198, 205-216 (1949).

30. Floro, J. A. et al. The dynamic competition between stress generation and relaxation mechanisms during coalescence of Volmer-Weber thin films. Journal of Applied Physics 89, 4886-4897 (2001).

31. Mo, Y.-W., Savage, D. E., Swartzentruber, B. S. \& Lagally, M. G. Kinetic pathway in StranskiKrastanov growth of Ge on Si(001). Phys. Rev. Lett. 65, 1020-1023 (1990).

32. Hong, W. et al. Persistent Step-Flow Growth of Strained Films on Vicinal Substrates. Phys. Rev. Lett. 95, 095501 (2005).

33. Ohring, M. Materials Science of Thin Films, Second Edition. (Academic Press, 2001).

34. Chambers, S. A. Epitaxial growth and properties of thin film oxides. Surface Science Reports 39, 105-180 (2000).

35. Martin, L. W., Chu, Y.-H. \& Ramesh, R. Advances in the growth and characterization of magnetic, ferroelectric, and multiferroic oxide thin films. Materials Science and Engineering: R: Reports 68, 89-133 (2010).

36. Matthews, J. W. \& Blakeslee, A. E. Defects in epitaxial multilayers: I. Misfit dislocations. Journal of Crystal Growth 27, 118-125 (1974).

37. Sickafus, K. E., Wills, J. M. \& Grimes, N. W. Structure of Spinel. Journal of the American Ceramic Society 82, 3279-3292 (1999).

38. Chandramohan, P., Srinivasan, M. P., Velmurugan, S. \& Narasimhan, S. V. Cation distribution and particle size effect on Raman spectrum of $\mathrm{CoFe}_{2} \mathrm{O}_{4}$. Journal of Solid State Chemistry 184, 89-96 (2011).

39. Goldman, A. Modern Ferrite Technology. (Springer, 2005).

40. Momma, K. \& Izumi, F. VESTA 3 for three-dimensional visualization of crystal, volumetric and morphology data. Journal of Applied Crystallography 44, 1272-1276 (2011).

41. Bozorth, R. M., Tilden, E. F. \& Williams, A. J. Anisotropy and Magnetostriction of Some Ferrites. Phys. Rev. 99, 1788 (1955).

42. Slonczewski, J. C. Origin of Magnetic Anisotropy in Cobalt-Substituted Magnetite. Phys. Rev. 110, 1341 (1958).

43. Dorsey, P. C., Lubitz, P., Chrisey, D. B. \& Horwitz, J. S. $\mathrm{CoFe}_{2} \mathrm{O}_{4}$ thin films grown on (100) MgO substrates using pulsed laser deposition. J. Appl. Phys. 79, 6338 (1996).

44. Chambers, S. A. et al. Molecular beam epitaxial growth and properties of $\mathrm{CoFe}_{2} \mathrm{O}_{4}$ on $\mathrm{MgO}\left(\begin{array}{lll}0 & 0 & 1\end{array}\right)$. Journal of Magnetism and Magnetic Materials 246, 124-139 (2002).

45. Dhakal, T. et al. Magnetic anisotropy and field switching in cobalt ferrite thin films deposited by pulsed laser ablation. J. Appl. Phys. 107, 053914 (2010).

46. Terzzoli, M. C., Duhalde, S., Jacobo, S., Steren, L. \& Moina, C. High perpendicular coercive field of $\mathrm{CoFe}_{2} \mathrm{O}_{4}$ thin films deposited by PLD. Journal of Alloys and Compounds 369, 209-212 (2004).

47. Yanagihara, H., Uwabo, K., Minagawa, M., Kita, E. \& Hirota, N. Perpendicular magnetic anisotropy in $\mathrm{CoFe} 2 \mathrm{O} 4(001)$ films epitaxially grown on $\mathrm{MgO}(001)$. J. Appl. Phys. 109, 07C122 (2011).

48. Thang, P. D., Rijnders, G. \& Blank, D. H. A. Stress-induced magnetic anisotropy of $\mathrm{CoFe} 2 \mathrm{O} 4$ thin films using pulsed laser deposition. Journal of Magnetism and Magnetic Materials 310, 2621-2623 (2007). 
49. Huang, W. et al. Epitaxial growth of the CoFe2O4 film on $\mathrm{SrTiO} 3$ and its characterization. Journal of Crystal Growth 300, 426-430 (2007).

50. Glazer, A. M. The classification of tilted octahedra in perovskites. Acta Crystallographica Section B Structural Crystallography and Crystal Chemistry 28, 3384-3392 (1972).

51. Goldschmidt, H. J. \& Rait, J. R. Silicates of the Perovskite Type of Structure. Nature 152, 356 (1943).

52. Michel, C., Moreau, J.-M., Achenbach, G. D., Gerson, R. \& James, W. J. The atomic structure of BiFeO3. Solid State Communications 7, 701-704 (1969).

53. Kubel, F. \& Schmid, H. Structure of a ferroelectric and ferroelastic monodomain crystal of the perovskite $\mathrm{BiFeO}_{3}$. Acta Crystallogr B Struct Sci 46, 698-702 (1990).

54. Teague, J. R., Gerson, R. \& James, W. J. Dielectric hysteresis in single crystal BiFeO3. Solid State Communications 8, 1073-1074 (1970).

55. Wang, J. et al. Epitaxial $\mathrm{BiFeO}_{3}$ Multiferroic Thin Film Heterostructures. Science 299, 1719-1722 (2003).

56. Neaton, J. B., Ederer, C., Waghmare, U. V., Spaldin, N. A. \& Rabe, K. M. First-principles study of spontaneous polarization in multiferroic $\mathrm{BiFeO}_{3}$. Phys. Rev. B 71, 014113 (2005).

57. Zavaliche, F. et al. Electrically Assisted Magnetic Recording in Multiferroic Nanostructures. Nano Letters 7, 1586-1590 (2007).

58. Kawasaki, M. et al. Atomic Control of the $\mathrm{SrTiO}_{3}$ Crystal Surface. Science 266, 1540 -1542 (1994).

59. Kabelac, J., Ghosh, S., Dobal, P. \& Katiyar, R. rf oxygen plasma assisted molecular beam epitaxy growth of $\mathrm{BiFeO}_{3}$ thin films on $\mathrm{SrTiO}_{3}$ (001). J. Vac. Sci. Technol. B 25, 1049 (2007).

60. Yang, H. et al. Rectifying current-voltage characteristics of $\mathrm{BiFeO}_{3} \mathrm{Nb}$-doped $\mathrm{SrTiO}_{3}$ heterojunction. Appl. Phys. Lett. 92, 102113 (2008).

61. Li, J. et al. Dramatically enhanced polarization in (001), (101), and (111) $\mathrm{BiFeO}_{3}$ thin films due to epitiaxial-induced transitions. Appl. Phys. Lett. 84, 5261 (2004).

62. Dupé, B. et al. Competing phases in $\mathrm{BiFeO}_{3}$ thin films under compressive epitaxial strain. Phys. Rev. $B$ 81, 144128 (2010).

63. Derighetti, B., Drumheller, J. E., Laves, F., Müller, K. A. \& Waldner, F. The space group of $\mathrm{LaAlO}_{3}$ below $720^{\circ} \mathrm{K}$, and the compounds $\mathrm{NdAlP}_{3}$ and $\mathrm{PrAlO}_{3}$ probably isomorphous at room temperature. Acta Crystallographica 18, 557-557 (1965).

64. Geller, S. \& Bala, V. B. Crystallographic studies of perovskite-like compounds. II. Rare earth alluminates. Acta Crystallographica 9, 1019-1025 (1956).

65. Zeches, R. J. et al. A Strain-Driven Morphotropic Phase Boundary in $\mathrm{BiFeO}_{3}$. Science 326, 977 -980 (2009).

66. Béa, H. et al. Evidence for Room-Temperature Multiferroicity in a Compound with a Giant Axial Ratio. Phys. Rev. Lett. 102, 217603 (2009).

67. Christen, H. M., Nam, J. H., Kim, H. S., Hatt, A. J. \& Spaldin, N. A. Stress-induced R-M $\mathrm{A}_{\mathrm{A}}-\mathrm{M}_{\mathrm{C}}-\mathrm{T}$ symmetry changes in $\mathrm{BiFeO}_{3}$ films. Phys. Rev. B 83, 144107 (2011).

68. Yang, J. C. et al. Orthorhombic $\mathrm{BiFeO}_{3}$. Phys. Rev. Lett. 109, 247606 (2012).

69. You, L. et al. Characterization and manipulation of mixed phase domains in highly-strained $\mathrm{BiFeO}_{3}$ thin films. arXiv:1112.6305 (2011). at <http://arxiv.org/abs/1112.6305>

70. Mazumdar, D. et al. Nanoscale Switching Characteristics of Nearly Tetragonal $\mathrm{BiFeO}_{3}$ Thin Films. Nano Lett. 10, 2555-2561 (2010).

71. Zheng, H. et al. Self-Assembled Growth of $\mathrm{BiFeO}_{3}-\mathrm{CoFe}_{2} \mathrm{O}_{4}$ Nanostructures. Advanced Materials 18, 2747-2752 (2006).

72. Zhan, Q. et al. Structure and interface chemistry of perovskite-spinel nanocomposite thin films. Appl. Phys. Lett. 89, 172902 (2006).

73. Zheng, H. et al. Controlling Self-Assembled Perovskite-Spinel Nanostructures. Nano Letters 6, 1401-1407 (2006).

74. Zhou, J., Qiu, Z. \& Liu, P. Electric and magnetic properties of $\mathrm{Pb}\left(\mathrm{Zr}_{0.52} \mathrm{Ti}_{0.48}\right) \mathrm{O}_{3}-\mathrm{CoFe}_{2} \mathrm{O}_{4}$ particle composite thin film on the $\mathrm{SrTiO}_{3}$ substrate. Materials Research Bulletin 43, 3514-3520 (2008). 
75. Zheng, H., Kreisel, J., Chu, Y.-H., Ramesh, R. \& Salamanca-Riba, L. Heteroepitaxially enhanced magnetic anisotropy in $\mathrm{BaTiO}_{3}-\mathrm{CoFe}_{2} \mathrm{O}_{4}$ nanostructures. Appl. Phys. Lett. 90, 113113 (2007).

76. Yan, L., Wang, Z., Xing, Z., Li, J. \& Viehland, D. Magnetoelectric and multiferroic properties of variously oriented epitaxial $\mathrm{BiFeO}_{3}-\mathrm{CoFe}_{2} \mathrm{O}_{4}$ nanostructured thin films. J. Appl. Phys. 107, 064106 (2010).

77. Jo, J. Y. et al. Structural Consequences of Ferroelectric Nanolithography. Nano Lett. 11, 3080-3084 (2011).

78. Oh, Y. S. et al. Quantitative determination of anisotropic magnetoelectric coupling in $\mathrm{BiFeO}_{3}-$ $\mathrm{CoFe}_{2} \mathrm{O}_{4}$ nanostructures. Appl. Phys. Lett. 97, 052902 (2010).

79. Dix, N. et al. On the strain coupling across vertical interfaces of switchable $\mathrm{BiFeO}_{3}-\mathrm{CoFe}_{2} \mathrm{O}_{4}$ multiferroic nanostructures. Appl. Phys. Lett. 95, 062907-062907-3 (2009).

80. Zavaliche, F. et al. Electric Field-Induced Magnetization Switching in Epitaxial Columnar Nanostructures. Nano Letters 5, 1793-1796 (2005).

81. Tan, Z. et al. Piezoelectric response of nanoscale $\mathrm{PbTiO}_{3}$ in composite $\mathrm{PbTiO}_{3}-\mathrm{CoFe}_{2} \mathrm{O}_{4}$ epitaxial films. Applied Physics Letters 93, 074101 (2008).

82. Levin, I., Slutsker, J., Li, J., Tan, Z. \& Roytburd, A. L. Accommodation of transformation strains in transverse multiferroic nanostructures $\mathrm{CoFe}_{2} \mathrm{O}_{4}-\mathrm{PbTiO}_{3}$. Applied Physics Letters 91, 062912 (2007).

83. Ding, L.-Y., Wu, F.-X., Chen, Y.-B., Gu, Z.-B. \& Zhang, S.-T. Controllable microstructures and multiferroic properties of $\mathrm{Pb}\left(\mathrm{Zr}_{0.53} \mathrm{Ti}_{0.47}\right) \mathrm{O}_{3}-\mathrm{CoFe}_{2} \mathrm{O}_{4}$ composite films. Applied Surface Science 257, 3840-3842 (2011).

84. Gao, X. et al. Microstructure and Properties of Well-Ordered Multiferroic $\mathrm{Pb}(\mathrm{Zr}, \mathrm{Ti}) \mathrm{O}_{3} / \mathrm{CoFe}_{2} \mathrm{O}_{4}$ Nanocomposites. ACS Nano 4, 1099-1107 (2011).

85. Ortega, N. et al. Multiferroic properties of $\mathrm{Pb}(\mathrm{Zr}, \mathrm{Ti}) \mathrm{O}_{3} / \mathrm{CoFe}_{2} \mathrm{O}_{4}$ composite thin films. J. Appl. Phys. 100, 126105 (2006).

86. Dix, N. et al. Selectable Spontaneous Polarization Direction and Magnetic Anisotropy in $\mathrm{BiFeO}_{3}-\mathrm{CoFe}_{2} \mathrm{O}_{4}$ Epitaxial Nanostructures. ACS Nano 4, 4955-4961 (2010).

87. Dix, N., Muralidharan, R., Varela, M., Fontcuberta, J. \& Sánchez, F. Mapping of the epitaxial stabilization of quasi-tetragonal $\mathrm{BiFeO}_{3}$ with deposition temperature. Applied Physics Letters 100, 122905-122905-4 (2012).

88. Liu, H.-J. et al. Epitaxial Photostriction-Magnetostriction Coupled Self-Assembled Nanostructures. ACS Nano 6, 6952-6959 (2012).

89. Lu, X. et al. Magnetoelectric Coupling in Ordered Arrays of Multilayered Heteroepitaxial $\mathrm{BaTiO}_{3} / \mathrm{CoFe}_{2} \mathrm{O}_{4}$ Nanodots. Nano Lett. 11, 3202-3206 (2011).

90. Vrejoiu, I., Morelli, A., Johann, F. \& Biggemann, D. Ordered $180^{\circ}$ ferroelectric domains in epitaxial submicron structures. Applied Physics Letters 99, 082906 (2011).

91. Ren, S., Briber, R. M. \& Wuttig, M. Diblock copolymer based self-assembled nanomagnetoelectric. Appl. Phys. Lett. 93, 173507 (2008).

92. Pan, Z., Alem, N., Sun, T. \& Dravid, V. P. Site-Specific Fabrication and Epitaxial Conversion of Functional Oxide Nanodisk Arrays. Nano Letters 6, 2344-2348 (2006).

93. Liu, W. et al. Understanding the formation of ultrafine spinel $\mathrm{CoFe}_{2} \mathrm{O}_{4}$ nanoplatelets and their magnetic properties. Journal of Applied Physics 112, 104306-104306-8 (2012).

94. Whitesides, G. M. \& Grzybowski, B. Self-Assembly at All Scales. Science 295, 2418 -2421 (2002).

95. Cheng, J. Y., Ross, C. A., Smith, H. I. \& Thomas, E. L. Templated Self-Assembly of Block Copolymers: Top-Down Helps Bottom-Up. Advanced Materials 18, 2505-2521 (2006).

96. Gherasimova, M., Hull, R., Reuter, M. C. \& Ross, F. M. Pattern level assembly of Ge quantum dots on Si with focused ion beam templating. Appl. Phys. Lett. 93, 023106 (2008).

97. Graham, J. F., Kell, C. D., Floro, J. A. \& Hull, R. Coupled effects of ion beam chemistry and morphology on directed self-assembly of epitaxial semiconductor nanostructures. Nanotechnology 22, 075301 (2011).

98. Guise, O. et al. Patterning of sub-10-nm Ge islands on Si(100) by directed self-assembly. Appl. Phys. Lett. 87, 171902 (2005). 
99. Sakamoto, T., Okada, K., Hattori, A. N., Kanki, T. \& Tanaka, H. Position-controlled functional oxide lateral heterostructures consisting of artificially aligned $(\mathrm{Fe}, \mathrm{Zn})_{3} \mathrm{O}_{4}$ nanodots and $\mathrm{BiFeO}_{3}$ matrix. Nanotechnology 23, 335302 (2012).

100. Comes, R. et al. Directed Self-Assembly of Epitaxial $\mathrm{CoFe}_{2} \mathrm{O} 4-\mathrm{BiFeO}_{3}$ Multiferroic Nanocomposites. Nano Lett. 12, 2367-2373 (2012).

101. Okada, K. et al. Three dimensional nano-seeding assembly of ferromagnetic $\mathrm{Fe} / \mathrm{LaSrFeO}_{4}$ nanohetero dot array. Journal of Applied Physics 112, 024320-024320-7 (2012).

102. Okada, K. \& Tanaka, H. Compositionally tunable three-dimensional nano-seeding assembly in Fe-LaSrFeO4 nanostructure. Journal of Applied Physics 113, 064317-064317-5 (2013).

103. Schwarzkopf, J. \& Fornari, R. Epitaxial growth of ferroelectric oxide films. Progress in Crystal Growth and Characterization of Materials 52, 159-212 (2006).

104. Eckstein, J. N. \& Bozovic, I. High-Temperature Superconducting Multilayers and Heterostructures Grown by Atomic Layer-By-Layer Molecular Beam Epitaxy. Annual Review of Materials Science 25, 679-709 (1995).

105. Schlom, D. G., Chen, L.-Q., Pan, X., Schmehl, A. \& Zurbuchen, M. A. A Thin Film Approach to Engineering Functionality into Oxides. Journal of the American Ceramic Society 91, 2429-2454 (2008).

106. Ino, S. Some New Techniques in Reflection High Energy Electron Diffraction (RHEED) Application to Surface Structure Studies. Japanese Journal of Applied Physics 16, 891-908 (1977).

107. Mannhart, J. \& Schlom, D. G. Oxide Interfaces-An Opportunity for Electronics. Science 327, 1607-1611 (2010).

108. Koinuma, H. \& Yoshimoto, M. Controlled formation of oxide materials by laser molecular beam epitaxy. Applied Surface Science 75, 308-319 (1994).

109. Christen, H. M. \& Eres, G. Recent advances in pulsed-laser deposition of complex oxides. Journal of Physics: Condensed Matter 20, 264005 (2008).

110. Ogale, S. B. Thin Films and Heterostructures for Oxide Electronics. (Springer, 2005).

111. Höbel, M., Geerk, J., Linker, G. \& Schultheiss, C. Deposition of superconducting YBaCuO thin films by pseudospark ablation. Appl. Phys. Lett. 56, 973 (1990).

112. Strikovski, M. \& Harshavardhan, K. S. Parameters that control pulsed electron beam ablation of materials and film deposition processes. Appl. Phys. Lett. 82, 853-855 (2003).

113. Christen, H. M. et al. Pulsed electron deposition of fluorine-based precursors for $\mathrm{YBa}_{2} \mathrm{Cu}_{3} \mathrm{O}_{7-\mathrm{x}}$ -coated conductors. Superconductor Science and Technology 18, 1168-1175 (2005).

114. Dediu, V. I. et al. Deposition of $\mathrm{MBa}_{2} \mathrm{Cu}_{3} \mathrm{O}_{7-\mathrm{x}}$ thin films by channel-spark method. Superconductor Science and Technology 8, 160-164 (1995).

115. Jiang, Q. D., Matacotta, F. C., Konijnenberg, M. C., Müller, G. \& Schultheiss, C. Deposition of $\mathrm{YBa}_{2} \mathrm{Cu}_{3} \mathrm{O}_{7-\mathrm{x}}$ thin films by channel-spark pulsed electron beam ablation. Thin Solid Films 241, 100102 (1994).

116. Chen, L., Xu, B., Chen, P., Chen, Z. \& Zhang, R. Controllable Formation of Particulates and the Effects on Superconductivity of $\mathrm{La}_{1.85} \mathrm{Sr}_{0.15} \mathrm{CuO}_{4}$ Film Grown by the Pulsed Electron Deposition Method. Journal of Superconductivity and Novel Magnetism 1-6 doi:10.1007/s10948-011-1399-7

117. Guo, Y. F. et al. Different parameters for the deposition of $\mathrm{La} 1.85 \mathrm{Sr} 0.15 \mathrm{CuO} 4$ and $\mathrm{Nd} 1.85 \mathrm{Ce} 0.15 \mathrm{CuO} 4$ superconducting films by the novel pulsed electron deposition technique. Physica C: Superconductivity 453, 64-69 (2007).

118. Guo, Y. F. et al. Doping tuned rectifying properties in $\mathrm{La}_{2-\mathrm{x}} \mathrm{Sr}_{\mathrm{x}} \mathrm{CuO}_{4} / \mathrm{Nb}: \mathrm{SrTiO}_{3}$ heterojunctions. Appl. Phys. Lett. 94, 143506-3 (2009).

119. Singh, B., Manoharan, S. S., Lim, S.-H. \& Salamanca-Riba, L. G. Magnetoresistance studies and optimization of deposition parameters of pulsed electron deposited $\mathrm{La}_{0.6} \mathrm{~Pb}_{0.4} \mathrm{Mn}_{0.8} \mathrm{Ru}_{0.2} \mathrm{O}_{3}$ thin films. The European Physical Journal Applied Physics 55, 20301 (2011).

120. Strikovski, M. D., Kim, J. \& Kolagani, S. H. in Springer Handbook of Crystal Growth (Dhanaraj, G., Byrappa, K., Prasad, V. \& Dudley, M.) 1193-1211 (Springer Berlin Heidelberg, 2010). at <http://www.springerlink.com/content/r7887375g5342303/> 
121. Choudhary, R. J. et al. Pulsed-electron-beam deposition of transparent conducting $\mathrm{SnO}_{2}$ films and study of their properties. Appl. Phys. Lett. 84, 1483-1485 (2004).

122. Béa, H. et al. Influence of parasitic phases on the properties of $\mathrm{BiFeO}_{3}$ epitaxial thin films. Applied Physics Letters 87, 072508-072508-3 (2005).

123. Kirkwood, D. Growth and Development of Meta-Multiferroic Systems using Pulsed Electron Deposition. (2010). at <http://search.proquest.com/docview/851701399/abstract?accountid=14678>

124. Murugesan, M., Obara, H., Nakagawa, Y., Kosaka, S. \& Yamasaki, H. Influence of MgO substrate annealing on the microwave properties of laser ablated $\mathrm{YBa}_{2} \mathrm{Cu}_{3} \mathrm{O}_{\mathrm{z}}$ thin films. Superconductor Science and Technology 17, 113-119 (2004).

125. Wi, J.-S. et al. Electron-beam lithography of $\mathrm{Co} / \mathrm{Pd}$ multilayer with hydrogen silsesquioxane and amorphous Si intermediate layer. J. Vac. Sci. Technol. B 24, 2616-2620 (2006).

126. Wi, J.-S. et al. Guided Formation of a Sub-10 nm Silicide Dot Array on an Area Patterned by Electron-Beam Lithography. Advanced Materials 19, 3469-3472 (2007).

127. Wi, J.-S. et al. Fabrication of Silicon Nanopillar Teradot Arrays by Electron-Beam Patterning for Nanoimprint Molds. Small 4, 2118-2122 (2008).

128. Hart, M. Bragg reflection x ray optics. Reports on Progress in Physics 34, 435-490-4 (1971).

129. Pietsch, U., Holy, V.\& Baumbach, T. High-Resolution X-Ray Scattering: From Thin Films to Lateral Nanostructures. (Springer, 2004).

130. Foner, S. Versatile and Sensitive Vibrating-Sample Magnetometer. Review of Scientific Instruments 30, 548-557 (1959).

131. Cullity, B. D. \& Graham, C. D. Introduction to Magnetic Materials. (J.W. Wiley and Sons, Inc., 2009).

132. Fultz, B. \& Howe, J. Transmission Electron Microscopy and Diffractometry of Materials. (Springer, 2009).

133. Schindelin, J. et al. Fiji: an open-source platform for biological-image analysis. Nature Methods 9, 676-682 (2012).

134. Binnig, G., Rohrer, H., Gerber, C. \& Weibel, E. Surface Studies by Scanning Tunneling Microscopy. Phys. Rev. Lett. 49, 57-61 (1982).

135. Binnig, G., Quate, C. F. \& Gerber, C. Atomic Force Microscope. Phys. Rev. Lett. 56, 930-933 (1986).

136. Hansma, P. K. et al. Tapping mode atomic force microscopy in liquids. Applied Physics Letters 64, 1738-1740 (1994).

137. Martin, Y. \& Wickramasinghe, H. K. Magnetic imaging by “"force microscopy”" with $1000 \AA$ resolution. Applied Physics Letters 50, 1455-1457 (1987).

138. Kolosov, O., Gruverman, A., Hatano, J., Takahashi, K. \& Tokumoto, H. Nanoscale Visualization and Control of Ferroelectric Domains by Atomic Force Microscopy. Phys. Rev. Lett. 74, 4309-4312 (1995).

139. Hidaka, T. et al. Formation and observation of $50 \mathrm{~nm}$ polarized domains in $\mathrm{PbZr}_{1-\mathrm{x}} \mathrm{Ti}_{\mathrm{x}} \mathrm{O}_{3}$ thin film using scanning probe microscope. Applied Physics Letters 68, 2358-2359 (1996).

140. Petz, C. Epitaxial Nanotemplates for Strain Modulated Carrier Confinement in Silicon. (2012). at <http://ir.lib.virginia.edu/catalog/libra-oa:637>

141. Rugar, D. et al. Magnetic force microscopy: General principles and application to longitudinal recording media. Journal of Applied Physics 68, 1169-1183 (1990).

142. Durkan, C., Welland, M. E., Chu, D. P. \& Migliorato, P. Probing domains at the nanometer scale in piezoelectric thin films. Phys. Rev. B 60, 16198-16204 (1999).

143. Kalinin, S. V. \& Bonnell, D. A. Imaging mechanism of piezoresponse force microscopy of ferroelectric surfaces. Phys. Rev. B 65, 125408 (2002).

144. Eng, L. M. et al. Nondestructive imaging and characterization of ferroelectric domains in periodically poled crystals. Journal of Applied Physics 83, 5973-5977 (1998). 
145. Eng, L. M., Güntherodt, H.-J., Schneider, G. A., Köpke, U. \& Muñoz Saldaña, J. Nanoscale reconstruction of surface crystallography from three-dimensional polarization distribution in ferroelectric barium-titanate ceramics. Applied Physics Letters 74, 233-235 (1999).

146. Kalinin, S. V. et al. Vector Piezoresponse Force Microscopy. Microscopy and Microanalysis 12, 206-220 (2006).

147. Rodriguez, B. J., Callahan, C., Kalinin, S. V. \& Proksch, R. Dual-frequency resonance-tracking atomic force microscopy. Nanotechnology 18, 475504 (2007).

148. Jesse, S., Baddorf, A. P. \& Kalinin, S. V. Switching spectroscopy piezoresponse force microscopy of ferroelectric materials. Applied Physics Letters 88, 062908 (2006).

149. Horcas, I. et al. WSXM: A software for scanning probe microscopy and a tool for nanotechnology. Review of Scientific Instruments 78, 013705-013705-8 (2007).

150. Huang, W. et al. Strain induced magnetic anisotropy in highly epitaxial $\mathrm{CoFe}_{2} \mathrm{O}_{4}$ thin films. Appl. Phys. Lett. 89, 262506 (2006).

151. Aziz, M. J. Film growth mechanisms in pulsed laser deposition. Appl. Phys. A 93, 579-587 (2008).

152. Gao, X. S. et al. Switching of magnetic anisotropy in epitaxial $\mathrm{CoFe}_{2} \mathrm{O}_{4}$ thin films induced by $\mathrm{SrRuO}_{3}$ buffer layer. J. Phys. D: Appl. Phys. 42, 175006 (2009).

153. Fischer, A., Kühne, H. \& Richter, H. New Approach in Equilibrium Theory for Strained Layer Relaxation. Phys. Rev. Lett. 73, 2712 (1994).

154. Kale, S. et al. Film thickness and temperature dependence of the magnetic properties of pulsedlaser-deposited $\mathrm{Fe}_{3} \mathrm{O}_{4}$ films on different substrates. Phys. Rev. B 64, 205413 (2001).

155. Margulies, D. T. et al. Origin of the Anomalous Magnetic Behavior in Single Crystal $\mathrm{Fe}_{3} \mathrm{O}_{4}$ Films. Phys. Rev. Lett. 79, 5162 (1997).

156. Fitzgerald, A. G. \& May, T. G. Defects in epitaxial ferrite films grown by chemical vapour deposition. Thin Solid Films 35, 201-213 (1976).

157. Ramos, A. V., Matzen, S., Moussy, J.-B., Ott, F. \& Viret, M. Artificial antiphase boundary at the interface of ferrimagnetic spinel bilayers. Phys. Rev. B 79, 014401 (2009).

158. Ramos, A. V. et al. Room temperature spin filtering in epitaxial cobalt-ferrite tunnel barriers. Appl. Phys. Lett. 91, 122107 (2007).

159. Hu, G., Harris, V. G. \& Suzuki, Y. Microstructure and magnetic properties of cobalt ferrite thin films. Magnetics, IEEE Transactions on 37, 2347-2349 (2001).

160. Nistor, M., Mandache, N. B. \& Perrière, J. Pulsed electron beam deposition of oxides thin films. Journal of Physics D: Applied Physics 41, 165205 (2008).

161. Wang, J. et al. Epitaxial BiFeO3 Multiferroic Thin Film Heterostructures. Science 299, 1719$1722(2003)$.

162. Hatt, A. J., Spaldin, N. A. \& Ederer, C. Strain-induced isosymmetric phase transition in $\mathrm{BiFeO}_{3}$. Phys. Rev. B 81, 054109 (2010).

163. Ederer, C. \& Spaldin, N. A. Effect of Epitaxial Strain on the Spontaneous Polarization of Thin Film Ferroelectrics. Phys. Rev. Lett. 95, 257601 (2005).

164. Damodaran, A. R. et al. Nanoscale Structure and Mechanism for Enhanced Electromechanical Response of Highly Strained $\mathrm{BiFeO}_{3}$ Thin Films. Advanced Materials 23, 3170-3175 (2011).

165. Comes, R., et al. "Electron Molecular Beam Epitaxy: Layer-by-layer Growth of Complex Oxides via Pulsed Electron-beam Deposition," Journal of Applied Physics, 113, 023303 (2013).

166. Kalinin, S. V. \& Bonnell, D. A. Screening Phenomena on Oxide Surfaces and Its Implications for Local Electrostatic and Transport Measurements. Nano Lett. 4, 555-560 (2004).

167. Liu, H., Comes, R., Pei, Y., Lu, J. \& Wolf, S. A. Structural, magnetic, and nanoscale switching properties of $\mathrm{BiFeO}_{3}$ thin films grown by pulsed electron deposition. Journal of Vacuum Science and Technology $B$, in press.

168. Mishra, R. K. \& Thomas, G. Surface energy of spinel. Journal of Applied Physics 48, 4576-4580 (1977). 
169. Chambers, S. A. Epitaxial Growth and Properties of Doped Transition Metal and Complex Oxide Films. Advanced Materials 22, 219-248 (2010).

170. Graziosi, P. et al. Conditions for the growth of smooth $\mathrm{La}_{0.7} \mathrm{Sr}_{0.3} \mathrm{MnO} 3$ thin films by pulsed electron ablation. arXiv:1212.6524 (2012). at <http://arxiv.org/abs/1212.6524>

171. Bucci, J. D., Robertson, B. K. \& James, W. J. The precision determination of the lattice parameters and the coefficients of thermal expansion of $\mathrm{BiFeO}_{3}$. Journal of Applied Crystallography 5, 187-191 (1972).

172. Chen, D.-X., Brug, J. A. \& Goldfarb, R. B. Demagnetizing factors for cylinders. IEEE Transactions on Magnetics 27, 3601-3619 (1991).

173. Hsieh, Y.-H. et al. Local Conduction at the $\mathrm{BiFeO}_{3}-\mathrm{CoFe}_{2} \mathrm{O}_{4}$ Tubular Oxide Interface. Advanced Materials 24, 4564-4568 (2012).

174. Rodriguez, B. J. et al. Spatially resolved mapping of ferroelectric switching behavior in selfassembled multiferroic nanostructures: strain, size, and interface effects. Nanotechnology 18, 405701 (2007).

175. Chang, H. et al. Watching domains grow: In-situ studies of polarization switching by combined scanning probe and scanning transmission electron microscopy. J. Appl. Phys. 110, 052014 (2011).

176. Liu, H., Comes, R., Pei, Y., Lu, J. \& Wolf, S. Structural, magnetic, and nanoscale switching properties of $\mathrm{BiFeO}_{3}$ thin films grown by pulsed electron deposition. arXiv:1301.5237 (2013). at <http://arxiv.org/abs/1301.5237>

177. Chu, Y. - $\mathrm{H}$ et al. Nanoscale Domain Control in Multiferroic $\mathrm{BiFeO}_{3}$ Thin Films. Advanced Materials 18, 2307-2311 (2006).

178. Chu, Y. - H et al. Domain Control in Multiferroic $\mathrm{BiFeO}_{3}$ through Substrate Vicinality. Advanced Materials 19, 2662-2666 (2007).

179. Comes, R., Gu, M., Khokhlov, M., Lu, J. \& Wolf, S. A. Microstructural and domain effects in epitaxial $\mathrm{CoFe}_{2} \mathrm{O}_{4}$ films on $\mathrm{MgO}$ with perpendicular magnetic anisotropy. Journal of Magnetism and Magnetic Materials 324, 524-527 (2012).

180. Owen, G. \& Rissman, P. Proximity effect correction for electron beam lithography by equalization of background dose. Journal of Applied Physics 54, 3573-3581 (1983).

181. Comes, R., Khokhlov, M., Liu, H., Lu, J. \& Wolf, S. A. Magnetic anisotropy in composite $\mathrm{CoFe}_{2} \mathrm{O}_{4}-\mathrm{BiFeO}_{3}$ ultrathin films grown by pulsed-electron deposition. Journal of Applied Physics 111, 07D914-07D914-3 (2012).

182. Rodriguez, B. J. et al. Spatially resolved mapping of ferroelectric switching behavior in selfassembled multiferroic nanostructures: strain, size, and interface effects. Nanotechnology 18, 405701 (2007).

183. Proksch, R. \& Kalinin, S. Piezoresponse Force Microscopy with Asylum Research AFMs. at <http://www.asylumresearch.com/Applications/PFMAppNote/PFM-ANHR.pdf>

184. Hruszkewycz, S. O., Holt, M. V., Tripathi, A., Maser, J. \& Fuoss, P. H. Framework for threedimensional coherent diffraction imaging by focused beam x-ray Bragg ptychography. Opt. Lett. 36, 2227-2229 (2011).

185. Backen, E. et al. Improved Pinning in YBCO Based Quasi-Multilayers Prepared by On- and OffAxis Pulsed Laser Deposition. IEEE Transactions on Applied Superconductivity 17, 3733 -3736 (2007).

186. Okada, K. \& Tanaka, H. Compositionally tunable three-dimensional nano-seeding assembly in $\mathrm{Fe}-\mathrm{LaSrFeO}_{4}$ nanostructure. Journal of Applied Physics 113, 064317-064317-5 (2013).

187. Assmann, E. et al. Oxide Heterostructures for Efficient Solar Cells. arXiv:1301.1314 (2013). at $<$ http://arxiv.org/abs/1301.1314>

188. Suntivich, J., May, K. J., Gasteiger, H. A., Goodenough, J. B. \& Shao-Horn, Y. A Perovskite Oxide Optimized for Oxygen Evolution Catalysis from Molecular Orbital Principles. Science 334, 1383-1385 (2011).

189. Okuda, T., Nakanishi, K., Miyasaka, S. \& Tokura, Y. Large thermoelectric response of metallic perovskites: $\mathrm{Sr}_{1-\mathrm{x}} \mathrm{La}_{\mathrm{x}} \mathrm{TiO}_{3}(0<\sim \mathrm{x}<\sim 0.1)$. Phys. Rev. B 63, 113104 (2001). 
190. Chuang, V. P., Gwyther, J., Mickiewicz, R. A., Manners, I. \& Ross, C. A. Templated SelfAssembly of Square Symmetry Arrays from an ABC Triblock Terpolymer. Nano Letters 9, 4364 4369 (2009).

191. Pan, Z., Alem, N., Sun, T. \& Dravid, V. P. Site-Specific Fabrication and Epitaxial Conversion of Functional Oxide Nanodisk Arrays. Nano Lett. 6, 2344-2348 (2006).

192. Pan, Z., Li, S., Wang, Z., Yu, M.-F. \& Dravid, V. P. Patterning-controlled morphology of spatially and dimensionally constrained oxide nanostructures. Applied Physics Letters 91, 143105143105-3 (2007).

193. Dufresne, E. A technique that might be useful [E-mail]. (2012).

194. Shen, Q., Umbach, C. C., Weselak, B. \& Blakely, J. M. X-ray diffraction from a coherently illuminated Si(001) grating surface. Phys. Rev. B 48, 17967-17971 (1993).

195. Wen, H. et al. Electronic Origin of Ultrafast Photoinduced Strain in $\mathrm{BiFeO}_{3}$. Phys. Rev. Lett. 110, 037601 (2013). 


\section{Appendix}

The following table lists all growth parameters for the samples presented in this thesis. Detailed descriptions of the step-by-step fabrication process can be found in my research notebooks. The sample code begins with E for "electron" in the pulsed electron deposition system, then the date in MMDDYY form, followed by a letter representing the order of deposition that day (A first, B second, etc). If multiple substrates were used in the same growth a dash followed by a number is included to denote the substrate.

\begin{tabular}{|c|c|c|c|c|c|c|c|c|c|c|}
\hline Sample Code & $\begin{array}{l}\text { Figure } \\
\text { in } \\
\text { Thesis }\end{array}$ & $\begin{array}{l}\text { Material } \\
\text { Deposited }\end{array}$ & Substrate & $\begin{array}{l}\text { Set } \\
\text { Temperature } \\
\left(\begin{array}{l}\left.{ }^{\circ} \mathrm{C}\right)\end{array}\right)\end{array}$ & $\begin{array}{l}\text { Calculated } \\
\text { Surface } \\
\text { Temperature } \\
\left(\begin{array}{ll}{ }^{\circ} & \text { C })\end{array}\right.\end{array}$ & $\begin{array}{l}\text { Operating } \\
\text { Pressure } \\
\text { (mTorr) }\end{array}$ & $\begin{array}{l}\text { Gas } \\
\text { Composition }\end{array}$ & $\begin{array}{l}\text { Pulse } \\
\text { Voltage } \\
(\mathrm{kV})\end{array}$ & $\begin{array}{l}\text { Pulse } \\
\text { Rate } \\
(\mathrm{Hz})\end{array}$ & $\begin{array}{l}\text { Number of } \\
\text { Pulses } \\
\text { (thousands) }\end{array}$ \\
\hline E120910B-1 & $\begin{array}{l}5-1 \text { to } \\
5-4\end{array}$ & $\mathrm{CoFe}_{2} \mathrm{O}_{4}$ & $\mathrm{MgO}$ & 802 & 600 & 18 & $100 \% \mathrm{O}_{2}$ & 12.5 & 10 & 100 \\
\hline E121010A-1 & $\begin{array}{l}5-1 \text { to } \\
5-4\end{array}$ & $\mathrm{CoFe}_{2} \mathrm{O}_{4}$ & $\mathrm{MgO}$ & 802 & 600 & 18 & $100 \% \mathrm{O}_{2}$ & 12.5 & 10 & 50 \\
\hline E121010B-1 & $\begin{array}{l}5-1 \text { to } \\
5-4 \\
\end{array}$ & $\mathrm{CoFe}_{2} \mathrm{O}_{4}$ & $\mathrm{MgO}$ & 802 & 600 & 18 & $100 \% \mathrm{O}_{2}$ & 12.5 & 10 & 200 \\
\hline E070812A & $5-5$ & $\mathrm{CoFe}_{2} \mathrm{O}_{4}$ & $\mathrm{SrTiO}_{3}$ & 700 & 515 & 12 & $100 \% \mathrm{O}_{2}$ & 9 & 8 & 40 \\
\hline E122711B & $5-5$ & $\mathrm{CoFe}_{2} \mathrm{O}_{4}$ & $\mathrm{SrTiO}_{3}$ & 700 & 515 & 17 & $100 \% \mathrm{O}_{2}$ & 12 & 5 & 25 \\
\hline E071612D & $5-8$ & $\mathrm{BiFeO}_{3}$ & $\mathrm{SrTiO}_{3}$ & 775 & 577 & 15 & $\begin{array}{l}70 \% \\
\mathrm{O}_{2} / 30 \% \mathrm{Ar}\end{array}$ & 13.5 & 5 & 15 \\
\hline E071712B-2 & $5-8$ & $\mathrm{BiFeO}_{3}$ & $\mathrm{LaAlO}_{3}$ & 775 & 577 & 15 & $\begin{array}{l}70 \% \\
\mathrm{O}_{2} / 30 \% \mathrm{Ar}\end{array}$ & 13.5 & 5 & 25 \\
\hline E072512B-2 & $5-6$ & $\mathrm{La}_{.72} \mathrm{Sr}_{.28} \mathrm{MnO}_{3}$ & $\mathrm{LaAlO}_{3}$ & 775 & 577 & 15 & $\begin{array}{l}70 \% \\
\mathrm{O}_{2} / 30 \% \mathrm{Ar}\end{array}$ & 9 & 5 & 15 \\
\hline E072612B-3 & $5-6$ & $\mathrm{La}_{.72} \mathrm{Sr}_{.28} \mathrm{MnO}_{3}$ & $\mathrm{LaAlO}_{3}$ & 775 & 577 & 15 & $\begin{array}{l}70 \% \\
\mathrm{O}_{2} / 30 \% \mathrm{Ar}\end{array}$ & 9 & 5 & 20 \\
\hline E072512B-1 & $5-7$ & $\mathrm{La}_{.72} \mathrm{Sr}_{.28} \mathrm{MnO}_{3}$ & $\mathrm{SrTiO}_{3}$ & 775 & 577 & 15 & $\begin{array}{l}70 \% \\
\mathrm{O}_{2} / 30 \% \mathrm{Ar}\end{array}$ & 9 & 5 & 15 \\
\hline E072612B-1 & $5-7$ & $\mathrm{La}_{.72} \mathrm{Sr}_{.28} \mathrm{MnO}_{3}$ & $\mathrm{SrTiO}_{3}$ & 775 & 577 & 15 & $\begin{array}{l}70 \% \\
\mathrm{O}_{2} / 30 \% \mathrm{Ar}\end{array}$ & 9 & 5 & 20 \\
\hline E072612C-1 & $5-9$ & $\begin{array}{l}\mathrm{BiFeO}_{3^{-}} \\
\mathrm{La}_{.72} \mathrm{Sr}_{.28} \mathrm{MnO}_{3}\end{array}$ & $\mathrm{SrTiO}_{3}$ & 775 & 577 & 15 & $\begin{array}{l}70 \% \\
\mathrm{O}_{2} / 30 \% \mathrm{Ar}\end{array}$ & $\begin{array}{l}9 \\
\text { (LSMO), } \\
12 \text { (BFO) }\end{array}$ & 5 & $\begin{array}{l}20 \\
\text { (LSMO), } \\
15 \text { (BFO) }\end{array}$ \\
\hline
\end{tabular}




\begin{tabular}{|c|c|c|c|c|c|c|c|c|c|c|}
\hline Sample Code & $\begin{array}{l}\text { Figure } \\
\text { in } \\
\text { Thesis }\end{array}$ & $\begin{array}{l}\text { Material } \\
\text { Deposited }\end{array}$ & Substrate & $\begin{array}{l}\text { Set } \\
\text { Temperature } \\
\left(\begin{array}{l}\circ \\
(\end{array}\right)\end{array}$ & $\begin{array}{l}\text { Calculated } \\
\text { Surface } \\
\text { Temperature } \\
\left({ }^{\circ} \mathrm{C}\right)\end{array}$ & $\begin{array}{l}\text { Operating } \\
\text { Pressure } \\
\text { (mTorr) }\end{array}$ & $\begin{array}{l}\text { Gas } \\
\text { Composition }\end{array}$ & $\begin{array}{l}\text { Pulse } \\
\text { Voltage } \\
(\mathrm{kV})\end{array}$ & $\begin{array}{l}\text { Pulse } \\
\text { Rate } \\
(\mathrm{Hz})\end{array}$ & $\begin{array}{l}\text { Number of } \\
\text { Pulses } \\
\text { (thousands) }\end{array}$ \\
\hline E062111A & $\begin{array}{l}6-1 \\
6-8, \\
6-9\end{array}$ & $\begin{array}{l}\mathrm{CoFe}_{2} \mathrm{O}_{4^{-}} \\
\mathrm{BiFeO}_{3} \\
\text { Composite }\end{array}$ & $\begin{array}{l}\mathrm{Nb}- \\
\mathrm{SrTiO}_{3}\end{array}$ & 825 & 622 & 16 & $100 \% \mathrm{O}_{2}$ & $\begin{array}{l}12.5 \\
\text { (CFO), } \\
12.5 \\
(\mathrm{BFO})\end{array}$ & $\begin{array}{l}4 \\
(\mathrm{CFO}), \\
5 \\
(\mathrm{BFO})\end{array}$ & $\begin{array}{l}40 \text { (CFO), } \\
50(\mathrm{BFO})\end{array}$ \\
\hline E081211A & $\begin{array}{l}6-1, \\
6-7\end{array}$ & $\begin{array}{l}\mathrm{CoFe}_{2} \mathrm{O}_{4^{-}} \\
\mathrm{BiFeO}_{3} \\
\text { Composite }\end{array}$ & $\begin{array}{l}\mathrm{Nb}- \\
\mathrm{SrTiO}_{3}\end{array}$ & 825 & 622 & 16 & $100 \% \mathrm{O}_{2}$ & $\begin{array}{l}12.5 \\
(\mathrm{CFO}), \\
12.5 \\
(\mathrm{BFO})\end{array}$ & $\begin{array}{l}3 \\
(\mathrm{CFO}), \\
5 \\
(\mathrm{BFO})\end{array}$ & $\begin{array}{l}12 \text { (CFO), } \\
20 \text { (BFO) }\end{array}$ \\
\hline E082111A & $\begin{array}{l}6-1, \\
6-7\end{array}$ & $\begin{array}{l}\mathrm{CoFe}_{2} \mathrm{O}_{4^{-}} \\
\mathrm{BiFeO}_{3} \\
\text { Composite }\end{array}$ & $\mathrm{SrTiO}_{3}$ & 825 & 622 & 16 & $100 \% \mathrm{O}_{2}$ & $\begin{array}{l}12.5 \\
\text { (CFO), } \\
12.5 \\
(\mathrm{BFO})\end{array}$ & $\begin{array}{l}4 \\
(\mathrm{CFO}), \\
5 \\
(\mathrm{BFO})\end{array}$ & $\begin{array}{l}160 \text { (CFO), } \\
200 \text { (BFO) }\end{array}$ \\
\hline E112612A & $\begin{array}{l}6-3 \text { to } \\
6-7, \\
6-10 \\
6-11\end{array}$ & $\begin{array}{l}\mathrm{CoFe}_{2} \mathrm{O}_{4^{-}} \\
\mathrm{BiFeO}_{3} \\
\text { Composite }\end{array}$ & $\begin{array}{l}\mathrm{Nb}- \\
\mathrm{SrTiO}_{3}\end{array}$ & 775 & 577 & 16 & $100 \% \mathrm{O}_{2}$ & $\begin{array}{l}11.5 \\
\text { (CFO), } \\
11.8 \\
(\mathrm{BFO})\end{array}$ & $\begin{array}{l}2.5 \\
(\mathrm{CFO}), \\
5 \\
(\mathrm{BFO})\end{array}$ & $\begin{array}{l}25 \text { (CFO), } \\
50 \text { (BFO) }\end{array}$ \\
\hline $\begin{array}{l}\text { E010413A } \\
\text { (template } \\
\text { E070912B-7) }\end{array}$ & $\begin{array}{l}7-6 \text { to } \\
7-18 \\
7-24 \\
7-25\end{array}$ & $\begin{array}{l}\mathrm{CoFe}_{2} \mathrm{O}_{4^{-}} \\
\mathrm{BiFeO}_{3} \\
\text { Composite }\end{array}$ & $\begin{array}{l}\mathrm{Nb}- \\
\mathrm{SrTiO}_{3}\end{array}$ & 775 & 577 & 16 & $100 \% \mathrm{O}_{2}$ & $\begin{array}{l}12 \\
\text { (CFO), } \\
11.8 \\
(\mathrm{BFO})\end{array}$ & $\begin{array}{l}2.5 \\
(\mathrm{CFO}), \\
5 \\
(\mathrm{BFO})\end{array}$ & $\begin{array}{l}37.5 \\
(\mathrm{CFO}), \\
75(\mathrm{BFO})\end{array}$ \\
\hline $\begin{array}{l}\text { E083111A } \\
\text { (template } \\
\text { E051911A-6) }\end{array}$ & $\begin{array}{l}7-19 \\
\text { to } \\
7-23 \\
7-26\end{array}$ & $\begin{array}{l}\mathrm{CoFe}_{2} \mathrm{O}_{4^{-}} \\
\mathrm{BiFeO}_{3} \\
\text { Composite }\end{array}$ & $\begin{array}{l}\mathrm{Nb}- \\
\mathrm{SrTiO}_{3}\end{array}$ & 825 & 622 & 16 & $100 \% \mathrm{O}_{2}$ & $\begin{array}{l}11.5 \\
(\mathrm{CFO}), \\
13.5 \\
(\mathrm{BFO})\end{array}$ & $\begin{array}{l}1 \\
(\mathrm{CFO}), \\
5 \\
(\mathrm{BFO})\end{array}$ & $\begin{array}{l}10 \text { (CFO), } \\
50 \text { (BFO) }\end{array}$ \\
\hline
\end{tabular}

